Institute of Pharmacognosy

University of Szeged

\title{
Synthesis of new, biologically active protoflavone derivatives
}

\author{
PhD Thesis \\ Balázs Dankó \\ Supervisor: \\ Dr. Attila Hunyadi \\ Institute of Pharmacognosy \\ University of Szeged, Szeged, Hungary \\ Co-supervisor: \\ Dr. Fang-Rong Chang \\ Dr. Yang-Chang Wu \\ Graduate Institute of Natural Products \\ Kaohsiung Medical University, Kaohsiung, Taiwan
}

Szeged

2019 


\section{Table of contents}

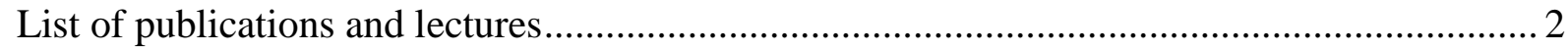

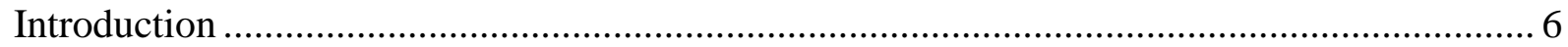

1.1. Protoflavonoids - chemistry and occurrence in Nature …........................................... 6

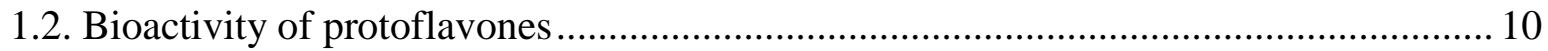

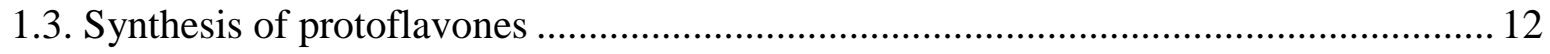

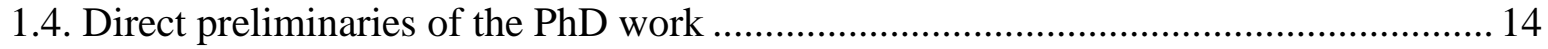

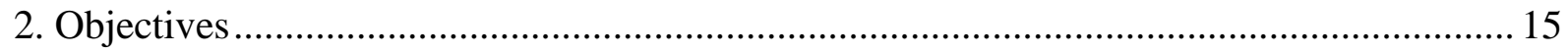

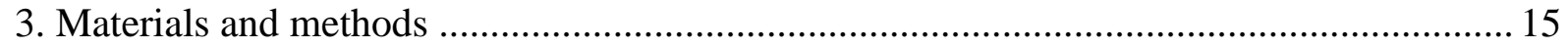

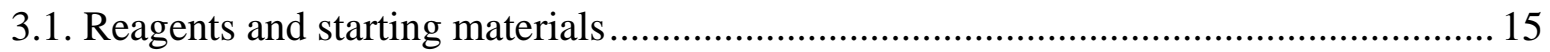

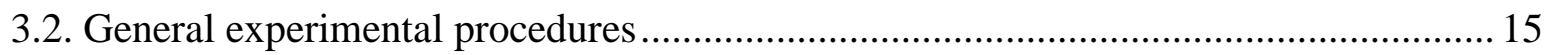

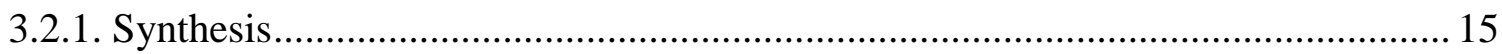

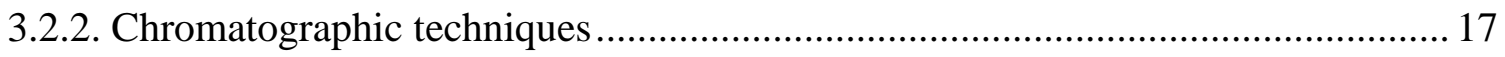

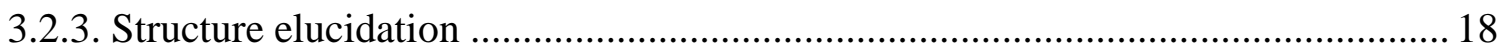

3.2.4. In silico studies on the formation of protoapigenone from apigenin ..................... 19

3.2.5. Experimental studies on the apigenin-protoapigenone transformation.................. 19

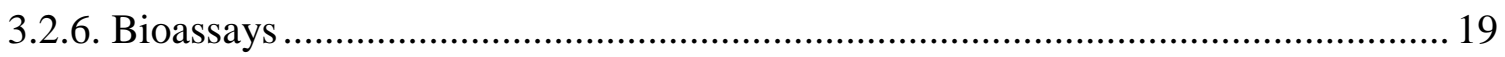

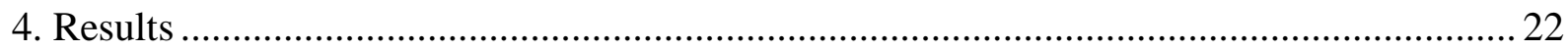

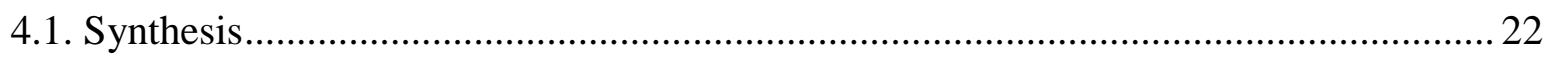

4.2. The role of $\mathrm{OH}$ radical scavenging in the formation of $\mathbf{1}$ from apigenin .................... 23

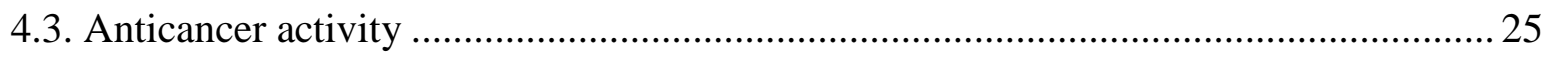

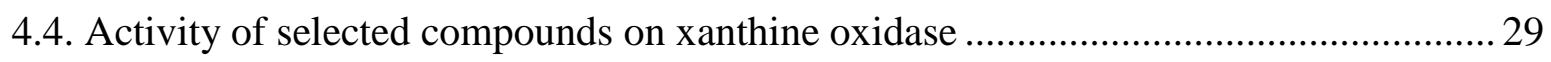

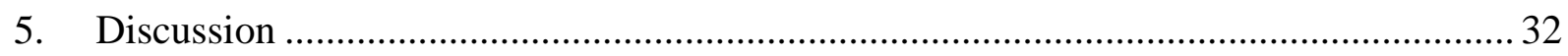

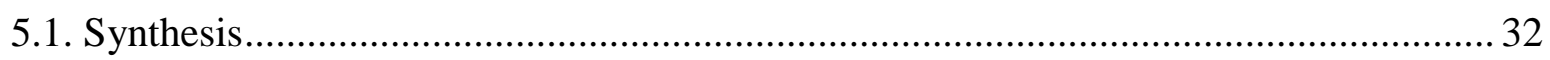

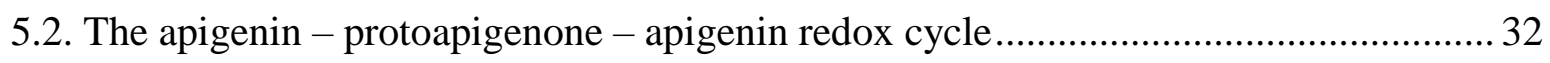

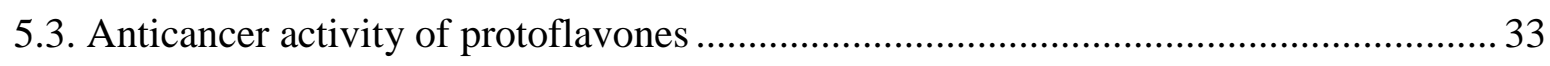

5.4. Xanthine oxidase inhibition by protoflavonoids .................................................... 37

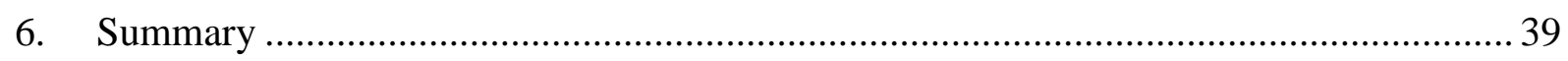

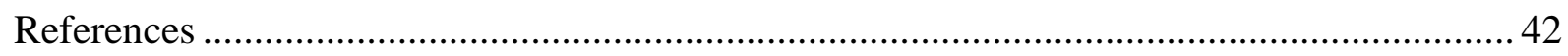

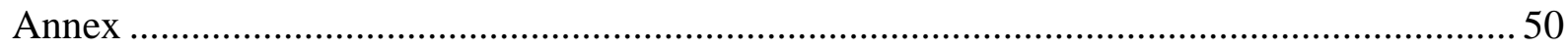




\section{List of publications and lectures}

Papers related to the thesis. In the text, roman numbers are used to refer to these papers.

I. Hunyadi A, Chuang DW, Danko B, Chiang MY, Lee CL, Wang HC, Wu CC, Chang FR, Wu YC; Direct Semi-synthesis of the Anticancer Lead-drug Protoapigenone from Apigenin, and Synthesis of Further New Cytotoxic Protoflavone Derivatives. PLoS ONE 2011, Vol. 6(8), pp. e23922. IF: 4.092

II. Danko B, Martins A, Chuang DW, Wang HC, Amaral L, Molnar J, Chang FR, Wu YC, Hunyadi A; In vitro Cytotoxic Activity of Novel Protoflavone Analogs - Selectivity against a Multi-drug Resistant Cancer Cell Line. Anticancer Res. 2012, Vol. 32, pp. 2863 2870.

\section{IF: $\mathbf{1 . 7 2 5}$}

III. Poor M, Li Y, Kunsagi-Mate S, Varga Zs, Hunyadi A, Dankó B, Chang FG, Wu YC, Kőszegi T; Protoapigenone Derivatives: Albumin Binding Properties and Effects on HepG2 Cells. Journal of Photochemistry and Photobiology B-Biology 2013, Vol 124, pp. 20-26. IF: 3.110

IV. Hunyadi A, Martins A, Danko B, Chuang D-W, Trouillas P, Chang F-R, Wu Y-C, Falkay G; Discovery of The First Non-planar Flavonoid That Can Strongly Inhibit Xanthine Oxidase: Protoapigenone 1'-o-propargyl ether. Tetrahedron Letters 2013, Vol 54(48), pp. 6529-6532. IF: $\mathbf{2 . 3 9 7}$

V. Hunyadi A, Martins A, Danko B, Chang FR, Wu YC; Protoflavones: a Class of Unusual Flavonoids As Promising Novel Anticancer Agents Phytochemistry Reviews, 2014, Vol 13, pp. 69-77. IF: $\mathbf{2 . 4 0 7}$

VI. Stankovic T, Danko B, Martins A, Dragoj M, Stojkovic, Isakovic A, Wang H-C, Wu Y-C, Hunyadi A, Pesic M. Lower Antioxidative Capacity of Multidrug-Resistant Cancer Cells Confers Collateral Sensitivity to Protoflavone Derivatives. Cancer Chemotherapy and Pharmacology, 2015, Vol 76(3), pp. 555-565. IF:2.824

VII. Danko B, Toth S, Martins A, Vagvolgyi M, Kusz N, Molnar J, Chang FR, Wu YC, Szakacs G, Hunyadi A. Synthesis and SAR Study of Novel Anticancer Protoflavone Derivatives Investigation of Cytotoxicity and Interaction with the ABCB1 and ABCG2 Multidrug Efflux Transporters. ChemMedChem, 2017, Vol. 12, pp 850-859. IF=3.225 


\section{Other papers}

VIII. Hunyadi A, Danko B, Boni, M, Militaru A, Alexandru T, Nastasa V, Andrei IR, Pascu M, Amaral L; Rapid, Laser-Induced Concersion of 20-Hydroxyecdysone and It's Diacetonide - Experimental Set-up of a System for Photochemical Transformation of Bioactive Substances. Anticancer Res. 2012, Vol 32(4), pp. 1291-1297. IF: 1.725

IX. Csupor D, Widowitz U, Blazsó G, Laczko-Zold E, Tatsimo J. S. N., Balogh A, Boros K, Danko B, Bauer R, Hohmann J; Anti-inflammatory Activities of Eleven Centaurea Species Occurring in the Carpathia Basin. Phytotherapy Res. 2013 Vol 27 pp540-544. IF: 2.650

X. Csupor D, Boros K, Danko B, Veres K, Szendrei K, Hohmann J; Rapid Identification of Sibutramine in Dietary Supplements Using a Stepwise Approach. Pharmazie 2013, Vol 68, pp. 15-18. IF:1.006

XI. Pascu ML, Danko B, Martins A, Jedlinszki N, Alexandru T, Nastasa V, Boni M, Militaru A, Andrei IR, Staicu A, Hunyadi A, Fanning S, Amaral L; Exposure of Chlorpromazine to 266nm Laser Beam Generates New Species with Antibacterial Properties: Contributions to a New Process for Drug Discovery. Plos One 2013, Vol 8(2), pp. e55767. IF:4.490

XII. Hunyadi A, Veres K, Danko B, Kele Z, Weber E, Hetenyi A, Zupko I, Hsieh TJ; In vitro Anti-diabetic Activity and Chemical Characterization of an Apolar Fraction of Morus alba Leaf Water Extract. Phytotherapy Research 2013, Vol 27(6) pp. 847-851. IF: 2.086

XIII. Armada AM, Alexandru T, Machado D, Danko B, Hunyadi A, Dinache A, Nastasa V, Boni M, Ramos J, Viveiros M, Molnar J, Pascu ML, Amaral L; The In Vitro Activity of Products Formed from Exposure of Chlorpromazine to a 266nm LASER Beam against Species of Mycobacteria of Human Interest. In Vivo 2013, Vol 27(5) pp. 605-610. IF: 1.148

XIV. Alexandru T, Armada A, Danko B, Hunyadi A, Militaru A, Boni M, Nastasa V, Martins A, Alexandru Viveiros M, Pascu ML, Molnar J, Amaral L; Biological Evaluation of Products Formed from the Irradiation of Chlorpromazine with a $266 \mathrm{~nm}$ Laser Beam. Biochemistry and Pharmacology 2013, Vol 2(1) pp. 1-4. IF: -

XV. Lai WC, Danko B, Csabi J, Kele Z, Chang FR, Pascu ML, Gáti T, Simon A, Amaral L, Tóth G, Hunyadi A. Rapid, Laser-Induced Conversion of 20-Hydroxyecdysone - a Followup Study on the Products Obtained Steroids 2014, Vol 89, pp. 56-62. IF: 2.639

XVI. Lai WC, Wu YC, Danko B, Cheng YB, Hsieh TJ, Martins A, Hohmann J, Hunyadi A, Chang FR. Bioactive Constituents of Cirsium japonicum var. australe. Journal of Natural Products 2014, Vol 77, pp. 1624-1631.

IF: 3.798 


\section{International conference presentations}

1. Hunyadi A, Danko B, Chuang DW, Chen SL, Martins A, Molnar J, Chang FR Wu YC; Microwave-assisted one-step synthesis and in vitro cytotoxic activity of protoapigenone and its 1'-O-alkyl derivatives. 25th Symposium on Natural Products, Nov. 6-7. 2010. Checheng, Taiwan,

2. Danko B, Lee JC, Chang FR, Wu YC, Hunyadi A; Synthesis and anti-HCV activity of 1'$O$-alkyl protoflavone derivatives. Trends in Natural Products Research: A PSE Young Scientist's Meeting, June 12-15. 2011. Kolymvari, Crete, Greece

3. Danko B, Boni M, Nastasa V, Pascu ML, Amaral L; Measurements on CPZ molecules modified by exposure to laser radiation and resulting biological activity against MDR Staphylococcus aureus. COST Action BM0701 6th Management Committee Meetings and Working Group Meeting December 6. 2011. Brussels, Belgium

4. Hunyadi A, Danko B, Chuang DW, Chang FR, Wu YC, Falkay G;Strong inhibition of xanthine oxidase by a non-planar flavonoid, protoapigenone 1'-O-propargylether. 12th Eurasia Conference on Chemical Sciences, Apr. 16-21. 2012. Corfu, Greece

5. Danko B, Bayach I, Fabre G, Trouillas P, Hunyadi A; In silico study of the redox properties of apigenin and protoapigenone - a midterm STSM progress report. COST Action CM0804, joint cost meeting, November 5-6, 2012, Salerno, Italy

6. Danko B, Martins A, Chang FR, Wu YC, Hunyadi A; Total synthesis of new protoflavone derivatives. COST Action CM1106, 2nd Working Group Meeting, September 19 - 20 2013, Warsaw, Poland

7. Danko B, Martins A, Amaral L, Molnar J, Chang FR, Wu YC, Hunyadi A; Synthesis of new MDR selective protoflavone derivatives. COST Action CM1106, 3nd Working Group Meeting, 27 - 28 March 2014, Budapest, Hungary

8. Kusz N, Danko B, Pinke Gy, Jakab G, Redei D, Hohmann J; Phytochemical investigation of Euphorbia dulcis and Euphorbia davidii. Trends in Natural Products Research, June 23 - 25 2014, Olomouc, Czech Republic

9. Stankovic T, Danko B, Milosevic Z, Bankovic J, Martins A, Molnar J, Amaral L, Hunyadi A, Pesic M; Selectivity of protoflavone derivatives towards human multi-drug resistant cancer cell lines. COST Action CM1106 3rd Working Group Meeting, March 27 - 28 2014, Budapest, Hungary 
10. Redei D, Kusz N, Forgo P, Danko B, Hohmann J; New jatrophane diterpenes from Euphorbia dulcis 22nd Conference on Isoprenoids, 7 - 10 September 2014, Prague, Czech Republic

11. Danko B, Martins A, Amaral L, Molnar J, Pesic M, Szakács G, Hunyadi A; Semi- and total-synthetic protoflavone derivatives as mdr selective anticancer agents 9th International Conference of Anticancer Research, 6 - 10 October 2014, Sithonia, Greece.

\section{Hungarian conference presentations}

1. Dankó B, Simon A, Báthori M; Különleges szerkezettel rendelkező szteroidok a Helleborus odorus-ból Kémiai Előadói Napok, 25-27 October 2010 Szeged.

2. Dankó B, Jin-Ching Lee, Fang-Rong Chang, Yang-Chang Wu, Hunyadi Attila; P-8 Synthesis and anti-HCV activity of 1'-O-alkyl protoflavone derivatives XII. Magyar Gyógynövény Konferencia, 5-7 May 2011. Szeged.

3. Hunyadi A, Dankó B, Martins A, Amaral L, Molnár J; Protoflavonok elöállítása és farmakológiai vizsgálata. Az MTA Alkaloidkémiai és Flavonoidkémiai munkabizottsága Elöadóülése. 12-13 May 2014. Balatonalmádi.

4. Kúsz N, Rédei D, Dankó B, Hohmann J, Euphorbia dulcis, az ígéretes diterpénforrás Egy szürővizsgálat eredménye. Fiatal Gyógynövénykutatók Fóruma, 14 February 2014. Budakalász.

5. Dankó B, Martins A, Chang FR, Wu YC, Hunyadi A. MDR szelektív rák ellenes hatással rendelkező protoflavon származékok előállítása. Congressus Pharmaceuticus Hungaricus XV, 10-12 April 2014 Budapest. 


\section{Introduction}

Flavonoids, probably the most widespread group of polyphenolic secondary metabolites in the Plant Kingdom, have long been recognized to play an important role in the prevention and/or treatment of many pathological conditions [1] [2]. Their chemical structure allows these compounds to participate in various redox reactions, including free radical scavenging, while due to their typically phenolic characteristics they can also specifically interact with several biochemical processes [3]. The common belief, which considers flavonoids in general as beneficial and healthy-to-consume plant metabolites, is certainly based on a large body of scientific evidence. Most common members of this compound family are present in fruits and vegetables, and they even have been suggested to be vitamins (vitamin P, coming from " "permeability") by the group of the Nobel-laureate Albert Szent-Györgyi [4] . Although they finally withdrew this theory, nowadays, more than 75 years later the most typical dietary flavonoids have a generally positive impact on human health. One has to note, however, that several classes of flavonoids can exert well defined, specific, sometimes strong bioactivities: isoflavones and prenylflavones are for example well known from their phytoestrogenic effect [5], and certain methoxylated flavones have been identified as potent antiviral agents [6], [3]. Many studies have suggested that flavonoids exhibit different biological effects, including antioxidant, anti-inflammatory, antiallergic, cytotoxic and antitumor activities. The work presented in this Thesis focuses on a rather unusual flavonoid group: protoflavonoids.

\subsection{Protoflavonoids - chemistry and occurrence in Nature}

Protoflavonoids are unusual natural flavonoid derivatives bearing a non-aromatic B-ring and a hydroxyl group in the C-1' position. Several further substituents can be present on the B-ring, but a 4'-oxo group, forming a p-quinol ring (that can be partially or fully saturated) is rather typical. The nomenclature of these compounds has had some ambiguity in the past. For example, the first flavonoid derivative named with a "proto"- prefix, protofarrerol (see below), was in fact the $4^{\prime}, 5^{\prime}$-dihydro derivative of what we would call today the protoflavanone analog of farrerol (6,8-dimethylnaringenin), and, accordingly, dihydroprotofarrerol has a fully saturated B-ring. Herein we will use a nomenclature that appears to be more and more generally accepted for the trivial names of protoflavonoids. A protoflavone is named based on the trivial name of the corresponding 4'-hydroxyflavone in a way that a "proto-" prefix is added in case a 4'-hydroxy group is present (as in protogenkwanin), and an additional "-one" ending shows if it has been oxidized to an oxo group (as in protogenkwanone). Accordingly, protoflavonoids 
with a 2,3-saturated basic skeleton are categorized as protoflavanones. This classification is presented in Fig. 1.
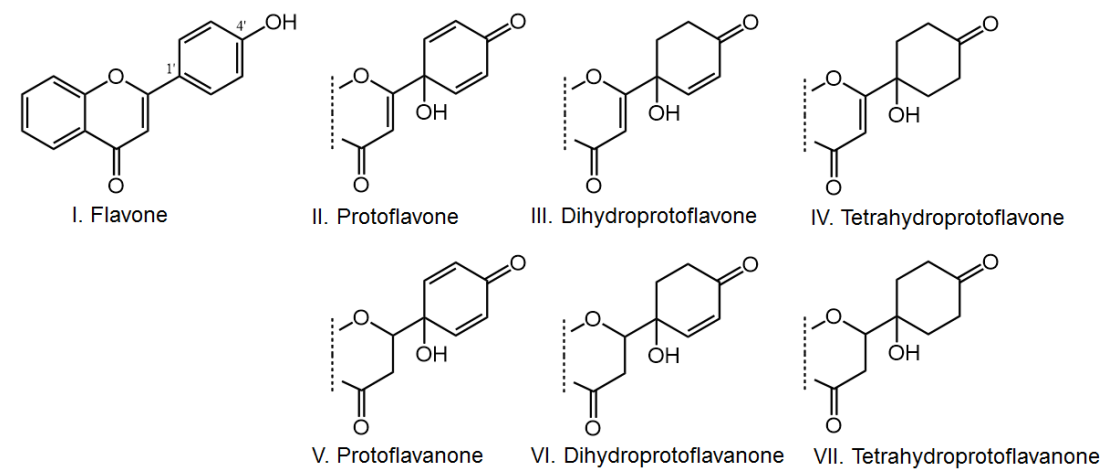

Figure 1. Types of protoflavonoid basic skeletons in comparison with that of a flavone

The first protoflavonoid, the protoflavanone type protofarrerol $(\mathbf{2 2})^{1}$ and dihydroprotofarrerol (26), were isolated from Leptorumohra miqueliana (Aspidiaceae) by Japanese scientists in the late 1960s, [7] [8] [9] ; and the 7-O- $\beta$-D-glucoside derivative of protofarrerol (23) was isolated from Monachosorum henryi (Pteridaceae) [10]. Further three protoflavanone derivatives were isolated from a Western African medicinal plant (Ongokea gore) [11], and, most recently, two more from a Piperaceae species, Piper carniconnectivum [12].

The first protoflavone was isolated from the fertile sprouts of Equisetum arvense and called protogenkwanin 4'-glucoside (14) [13]. This was soon followed by reporting the isolation of its aglycone, protogenkwanin (13), from the same source [14]. The two hydroxyl groups of this compound on the B ring at positions 1' and 4' were found to be trans oriented by means of X-ray crystallography (15), and similar orientation was found both for the partially [16] and the completely saturated B ring containing protogenkwanin analogs [17]. On the other hand, existence of the 1',4'-cis form in the dihydroprotogenkwanin series was also argued by Pouny et al. (2011) [18]. Structures of the known natural protoflavones are shown in Figure 2. ; occurrence of these compounds in plant species is summarized in Table 1 Although the biosynthesis of these compounds has not been investigated, a possible way for their production was suggested from the corresponding 4'-hydroxyflavone (i.e. apigenin or genkwanin) by oxidation. This step would then be followed by reduction of the 4'-oxo group and/or saturation of either one or both double bond(s) in the already non-aromatic B-ring [19]. Interestingly, the formation of derivatives with further hydroxylation on a partially saturated B-ring seems to

\footnotetext{
${ }^{1}$ As a compromise, a chemical logic was followed in numbering the protoflavonoids (see Fig. 2, page 8) rather than considering appearance in the text (i.e. the order of discovery). Accordingly, the best-studied protoflavone (protoapigenone), which also served as the ,archetype” of many of our compounds, was named as compound $\mathbf{1}$, leading to the not-in-order appearance of compound numbers within the text.
} 
occur via a non-specific way: 2'-hydroxy-2',3'-dihydroprotoapigenone (3) was isolated from Equisetum fluviatile as a racemate [18], and both enantiomers were also found to be present in Thelypteris torresiana [20]; [21]. The possible role of non-specific oxidative processes in the formation of protoflavones is also highlighted by a recent study reporting the first protoflavone derivative of an isoflavone, daidzeine. Interestingly, this compound, protodaidzeone, was obtained by the chlorophyll-catalyzed oxidation of daidzeine upon exposure with visible light [22]; similar processes can certainly take place also in living plants.

As per the above, it is not surprising that protoflavonoids have recently been reported also from plant species other than ferns, namely from Apium graveolens [23] and Piper carniconnectivum [24]. Accordingly, there seems to be a high chance that similar compounds can be found in several further plant species containing high amounts of flavonoids. Structures of the known natural protoflavonoids are presented in Fig. 2, their respective sources are given in Table 1.<smiles>[R]c1cc(O)c2c(=O)cc(C3(O)C=CC(=O)C=C3)oc2c1</smiles>

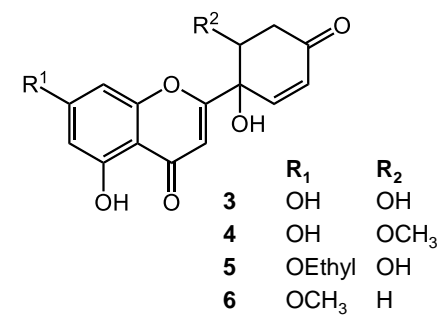<smiles></smiles><smiles>COc1cc(O)c2c(=O)cc(C3(O)C=CC(O)CC3)oc2c1</smiles><smiles>[R]c1cc(O)c2c(=O)cc(C3([R])C([R])CC(=O)CC3[R])oc2c1</smiles><smiles>[R]c1cc(O)c2c(=O)cc(C3(O)C=CC([R])C=C3)oc2c1</smiles>

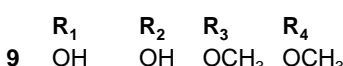
90

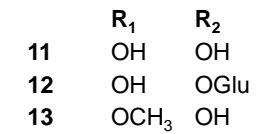

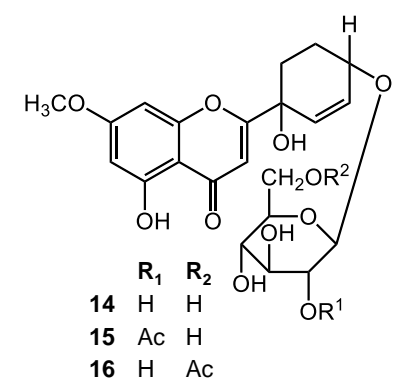

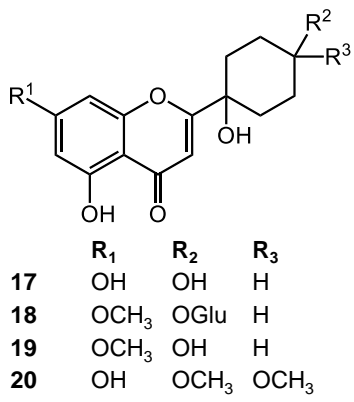<smiles>[R]c1c([R])c(O)c2c(c1[R])OC(C1(O)CCC(=O)CC1)CC2=O</smiles>

$\begin{array}{lll}\mathbf{R}_{1} & \mathbf{R}_{2} & \mathbf{R}_{3} \\ \mathrm{H} & \mathrm{OCH}_{3} & \mathrm{OCH}_{3}\end{array}$ $\begin{array}{lllll}21 & \mathrm{H} & \mathrm{OCH}_{3} & \mathrm{OCH}_{3} \\ 22 & \mathrm{CH}_{3} & \mathrm{OH} & \mathrm{CH}_{3}\end{array}$ $23 \mathrm{CH}_{3}$ OGlu $\mathrm{CH}_{3}$<smiles>[R]c1c([R])c(O)c2c(c1[R])OC([C@]1(O)CCC(=O)CC1[R])CC2=O</smiles>
$\begin{array}{llll}\mathbf{R}_{1} & \mathbf{R}_{2} & \mathbf{R}_{3} & \mathbf{R}_{4} \\ \mathrm{OCH}_{3} & \mathrm{OCH} & \mathrm{H} & \mathrm{OH}\end{array}$ $25 \mathrm{H} \quad \mathrm{OCH}_{3} \mathrm{H} \quad \mathrm{H}$ $26 \mathrm{CH}_{3} \quad \mathrm{OH} \quad \mathrm{CH}_{3} \mathrm{H}$<smiles>[R]C1([R])CCC(O)(c2cc(=O)c3c(O)cc(OC)cc3o2)CC1</smiles><smiles>CC1(C)C=Cc2c(cc3c(c2O)C(=O)CC(C2(O)C=CC(=O)CC2)O3)O1</smiles>

Figure 2. Structures of natural protoflavones (1-20) and protoflavanones (21-30) isolated from plants. 
Table 1. Occurrence of protoflavones in plants

\begin{tabular}{|c|c|c|}
\hline Plant Species & Protoflavones and protoflavanones identified a & Reference \\
\hline \multicolumn{3}{|l|}{ Ferns } \\
\hline \multirow[t]{2}{*}{ Equisetum arvense } & protogenkwanin 4'-O-ß-D-glucoside (14) & (25) \\
\hline & protogenkwanin (13) & (26) \\
\hline $\begin{array}{l}\text { Pseudophegopteris } \\
\text { hirtirachis }\end{array}$ & $\begin{array}{l}\text { protogenkwanone (2); tetrahydroprotogenkwanone (10); tetrahydroprotogenkwanin } \\
\text { (19); protogenkwanin 4'-O- } \beta \text {-D-glucoside (14) }\end{array}$ & (17) \\
\hline $\begin{array}{l}\text { Pseudophegopteris } \\
\text { subaurita }\end{array}$ & $\begin{array}{l}\text { protogenkwanone (2); tetrahydroprotogenkwanone (10); protogenkwanin 4'-O- } \beta \text {-D- } \\
\text { glucoside (14); protogenkwanin 4'-O-(2-O-acetyl)- } \beta \text {-D-glucoside (15); } \\
\text { protogenkwanin 4'-O-(6-O-acetyl)- } \beta \text {-D-glucoside (16) }\end{array}$ & ; \\
\hline $\begin{array}{l}\text { Pseudophegopteris } \\
\text { bukoensis }\end{array}$ & $\begin{array}{l}\text { protogenkwanin 4'-O-B-D-glucoside (14); protogenkwanin 4'-O-(2-O-acetyl)-- } \beta \text {-D- } \\
\text { glucoside (15); protogenkwanin 4'-O-(6-O-acetyl)- } \beta \text {-D-glucoside (16) }\end{array}$ & (17) \\
\hline \multirow[t]{2}{*}{ Equisetum fluviatile } & protoapigenin 4'-O-ß-D-glucoside (12) & (27) \\
\hline & 2',3'-dihydroprotogenkwanone (6); (+/-)2'-hydroxy-2',3'-dihydroprotoapigenone ${ }^{\mathrm{b}}$ (3) & (18) \\
\hline Equisetum debile & protoapigenone (1) & (18) \\
\hline Cyclosorus falcilobus & protoapigenin (11); protoapigenin 4'-O- $\beta$-D-glucoside (12) & (18) \\
\hline Cyclosorus parasiticus & protoapigenone (1) & (18) \\
\hline $\begin{array}{l}\text { Phegopteris decursive- } \\
\text { pinnata }\end{array}$ & protogenkwanone (2); 5',6'-dihydroprotogenkwanone (6); & (18) \\
\hline Phegopteris connectilis & 5',6'-dihydroprotogenkwanin (8) & (16) \\
\hline \multirow{6}{*}{$\begin{array}{l}\text { Thelypteris or } \\
\text { Macrothelypteris } \\
\text { torresianac }\end{array}$} & $\begin{array}{l}\text { protoapigenin (11); protoapigenin 4'-O-ß-D-glucoside (12); protoapigenone (1); 2'- } \\
\text { methoxy-2',3'-dihydroprotoapigenone (4) }\end{array}$ & (28) \\
\hline & flavotorresin (20) & (19) \\
\hline & 2'-hydroxy-2',3'-dihydroprotoapigenone acetonide (7) & (29) \\
\hline & 2',6'-dimethoxy-tetrahydroprotoapigenone (9) & (30) \\
\hline & tetrahydroprotoapigenine (17); (+)2'-hydroxy-2',3'-dihydroprotoapigenone (3) ${ }^{\mathrm{b}}$ & (20) \\
\hline & $(-) 2^{\prime}-$-hydroxy-2',3'-dihydroprotoapigenone (3) & (21) \\
\hline $\begin{array}{l}\text { Macrothelypteris } \\
\text { oligophlebia }\end{array}$ & protoapigenone (1); protoapigenin 4'-O-B-D-glucoside (12) & (31) \\
\hline \multirow[t]{3}{*}{$\begin{array}{l}\text { Macrothelypteris } \\
\text { viridifrons }\end{array}$} & 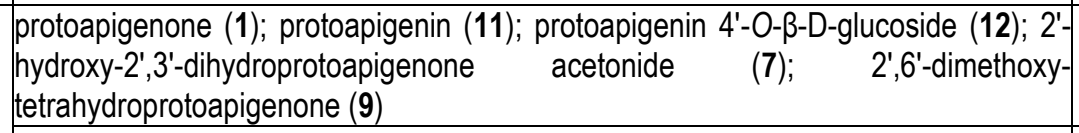 & (32) \\
\hline & 7-ethoxy-2'-hydroxy-2',3'-dihydroprotoapigenone (5) & (33) \\
\hline & $\begin{array}{l}\text { tetrahydroprotoapigenin (17); tetrahydroprotoapigenin 4'-O-ß-D-glucoside (18); 2'- } \\
\text { hydroxy-2',3'-dihydroprotoapigenone (3); 2'-methoxy-2',3'-dihydroprotoapigenone (4) }\end{array}$ & (34) \\
\hline Ongokea gore & \begin{tabular}{|l|} 
\\
(2S)- \\
ongokein-4'-one (25), (2S)-4',4'-dimethoxy-ongokein (27), \\
(2S)-cis-4'-hydroxy-ongokein (28), and (2S)-trans-4'-hydroxy-ongokein (29).
\end{tabular} & (36) \\
\hline \multirow{2}{*}{$\begin{array}{l}\text { Leptorumohra } \\
\text { miqueliana }\end{array}$} & protofarrerol (22) & (8) \\
\hline & dihydroprotofarrerol (26) & (7) \\
\hline Monachosorum henryi & protofarrerol 7-O-ß-D-glucopyranoside (23) & $(10)$ \\
\hline \begin{tabular}{|l||} 
Steganotaenia \\
araliacea
\end{tabular} & protosteganoflavanone (30) & (37) \\
\hline \multicolumn{3}{|l|}{ Other Species } \\
\hline Apium graveolens & protoapigenin 4'-O-ß-D-glucoside (12) & (23) \\
\hline \multicolumn{2}{|c|}{ 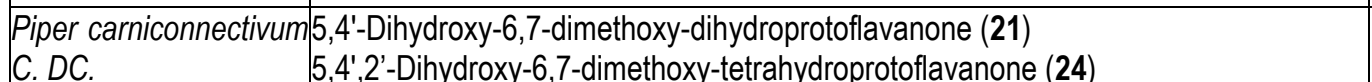 } & (35) \\
\hline
\end{tabular}

${ }^{\text {a }}$ Several compounds listed in this table were reported with their IUPAC names; for a more clear interpretation here they are presented according to the nomenclature described above

${ }^{\mathrm{b}} \mathrm{OH}$ or $\mathrm{OCH}_{3}$ groups present at $\mathrm{C}-20$ in derivatives with partially saturated B-ring are cis to the 10-OH group

${ }^{\mathrm{c}}$ Depending on the botanical definition of Thelypteridaceae, the species can be assigned to different genera; both names were used in protoflavone related publications 


\subsection{Bioactivity of protoflavones}

\section{Anticancer activity}

In several cases, the isolation of new natural protoflavones was reported together with bioactivity data, typically concerning their in vitro cytotoxic or anti-proliferative activity against various cancer cell lines. To date, however, protoapigenone (1) is by far the most deeply studied protoflavone. This compound and a number of its analogs were shown to exert strong cytotoxic activity in a wide variety of cancer cells including breast, ovarian, cervical, prostate, lung, liver, etc. ( [28] [19] [38] [39] [40] [41] [42]. Chang and co-authors also showed that protoapigenone (1) exerted strong cytotoxicity on ovarian cancer cell lines MDAH-2774 and SKOV3 but not on immortalized non-cancer ovarian epithelial cells [39]. Moreover, several studies showed that this compound induces apoptosis both in vitro and in vivo, without significantly damaging healthy tissues in the latter case [39] [40] [43].

\section{The mechanism of the anticancer activity}

Concerning the mechanism of action, several pathways were found to be involved in the anticancer activity of protoflavones, and mainly of protoapigenone (1)

Based on the chemical structure of the $p$-quinol B ring, protoflavones seem to be capable for participating in various redox reactions. Indeed, apoptosis induced by protoapigenone (1) and other synthetic analogs were shown to be correlated with the induction of oxidative stress within the cancer cells by generating reactive oxygen species (ROS) [44] [53] [33] [21]. In connection to this, inhibition of the enzyme glutathione S-transferase $\pi$ was also observed, even though it is still to be determined if this was a direct effect on the enzyme or rather a result of the increase in ROS within the cell [53]. Studies on breast cancer cells showed that protoapigenone treatment led to increased levels of ROS, required for activation of MAPK involved in the mitochondria-mediated apoptosis induced by this compound [53]. Similar observations were made when other protoflavonoids, WYC0209 [44], DHEC [34] and DEDC [21] were tested for their apoptotic effect on DU145 postate cancer, human colon HT-29 cancer and human neuroblastoma SH-SY5Y cell lines, respectively. In fact, WYC0209 was shown to increase intracellular generation of ROS, followed by DNA damage induction and activation of Chk1/Chk2 which led to cell cycle arrest at $\mathrm{S}$ and G2/M phases [44], and resulted in a stronger cytotoxic effect than that of protoapigenone (1) [39]. Due to the important role of ROS in the activity of protoflavones, it is currently hard to make a sound judgment on which of the 
previously mentioned pathways are subject to specific changes and which would be nonspecifically affected by the oxidative stress; further research will be necessary to clarify this.

DNA damage in lung [41] and prostate cancer cell lines [44] treated by protoapigenone or one of its synthetic analogs (WYC0209, see Fig. 3; page 13) was also observed. Damage of DNA is an important and frequent outcome during cancer treatment. In fact, inhibition of DNA damage response mechanisms is an attracting novel target for enhancing tumor chemosensitivity [45] [46]. Remarkably, a recent study showed that protoapigenone (1) and WYC0209 can significantly interact with the ATR-dependent signaling pathways by inhibiting the phosphorylation of Chk2 and Chk1 kinases [43] that play a crucial role in DNA damage response [47]. According to this, it was shown that these protoflavones themselves apparently do not directly damage DNA, the underlying mechanism is rather a specific inhibition of its repair. As expected, this inhibition could effectively sensitize the cancer cells to the DNA damaging anticancer drug cisplatin, resulting in significantly stronger combined activities as compared to those of the compounds administered alone [43]. These results suggest that future development of protoflavones might reasonably be re-directed towards the optimization of this highly promising chemo-sensitizing activity, in order to obtain valuable new options for combination chemotherapy. The synthetic WYC0209 deserves particular attention; this compound was as much as 10 times stronger than protoapigenone (1) in some of the aforementioned activities.

Apoptosis induction was shown to be mitogen-activated protein kinase (MAPK)-dependent in both human prostate [39] and breast cancer cells [44], also involving the activation of c-Jun $\mathrm{NH}_{2}$-Terminal Kinase (JNK). Related apoptotic pathways were also identified using ovarian cancer cell lines [40]: activation of caspase-3, cleavage of PARP and decreasing of Bcl-xL and Bcl-2 protein levels were found, accompanied by membrane alterations and nuclear fragmentations at different stages of the cell cycle. Protoapigenone (1) was also shown to interfere with the cell cycle inducing its arrest at $S$ and $\mathrm{G}_{2} / \mathrm{M}$ phases [39] [40] [41] Both in human ovarian [39] and prostate cancer cells [40], the cell cycle arrest was correlated with alterations in the expression level of Cdk2, Cyclin B1 and Cdc25C, well known cell cycle regulators that actually lead to a higher cytotoxic effect of protoapigenone (1) at this stages of the cell cycle. In human breast cancer cells [44] loss of the mitochondrial membrane potential was also observed and linked with the persistent activation of MAPK upon treatment with protoapigenone (1). 


\section{Other Bioactivities}

Protoapigenone (1) was shown to inhibit the expression of Epstein-Barr virus (EBV) lytic proteins in P3HR1 cells, preventing EBV lytic proliferation at doses well below those which exerted cytotoxic activity on the host cells [54]. This study also showed that the use of protoflavones is not limited to their anticancer activity; these flavonoids apparently represent a class of bioactive compounds with several further potential effects yet to be discovered.

\subsection{Synthesis of protoflavones}

The first total synthetic procedure of protoflavones was reported by Lin et al. in 2007 [38]. Protoapigenone and several synthetic protoflavones were synthetized by the Taiwanese research group as potential antitumor agents. After difficulties in carrying out the direct semisynthesis of $\mathbf{1}$ from apigenin, methoxymethyl-diprotected trihydroxyacetophenones and 4benzyloxybenzaldehyde as starting materials were utilized to prevent the A-ring of 2 from oxidation, and, after removal of the benzyl protecting group by catalytic hydrogenation, oxidative de-aromatization was performed by [bis(trifluoroacetoxy)iodo]benzene (commonly referred to as PIFA) in the presence of 0.2 eq. of TEMPO, a commercially available free radical catalyst in order to obtain the $p$-quinol moiety on the B-ring. Finally, 1 was successfully obtained after the removal of the methoxymethyl protecting group. The procedure was very long and required chromatographic purification at nearly all steps, resulting in as low as 3.3\% final isolated yield of $\mathbf{1}$. Scheme 1 shows an outline of this procedure, including reaction times and isolated yields of each reaction step.

Scheme 1. Total synthesis of protoapigenone reported by Lin et al. [28]

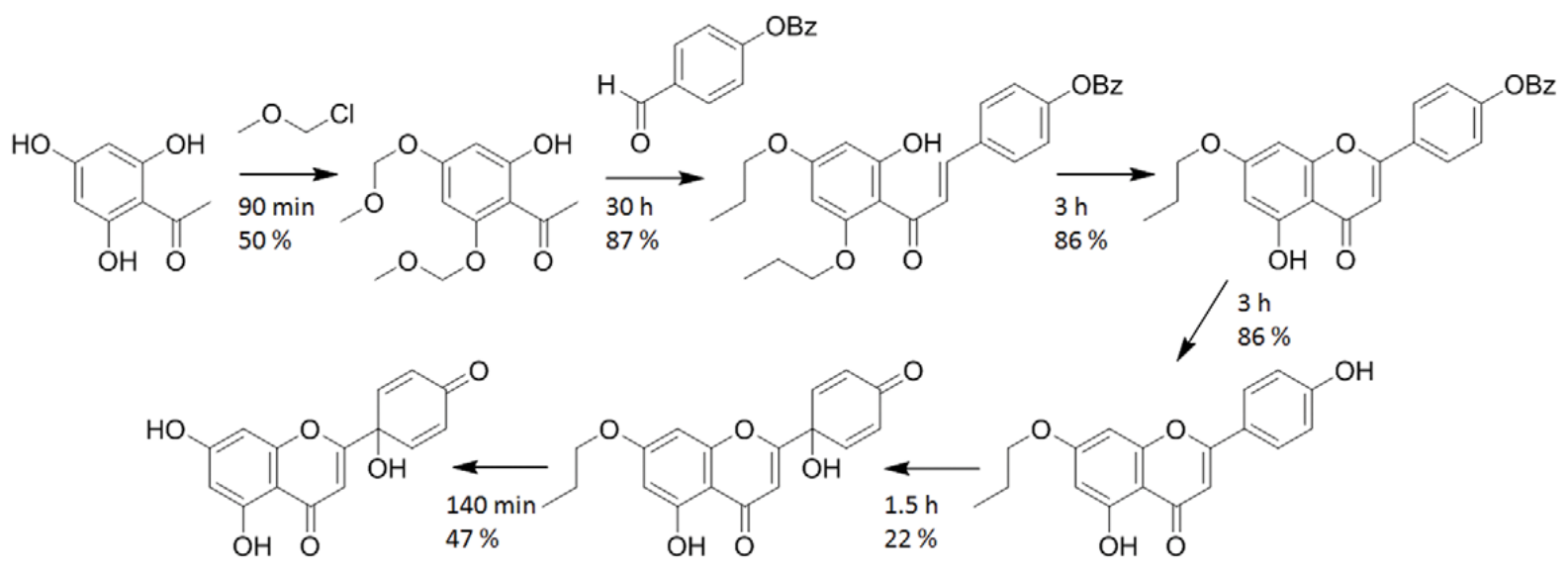


Scheme 2. Semi synthesis of protoapigenone and its $1-O$-alkyl ethers reported by Hunyadi et al. [42]<smiles>O=c1cc(-c2ccc(O)cc2)oc2cc(O)cc(O)c12</smiles><smiles>[R]C1(c2cc(=O)c3c(O)cc(O)cc3o2)C=CC(=O)C=C1</smiles>

Despite the difficulties in the total synthesis of $\mathbf{1}$, the above-referred work also led to the discovery of a synthetic derivative much stronger than protoapigenone, WYC0209 [38]. This compound, originated from 4'-hydroxy- $\beta$-naphthoflavone, was considered as one of the most promising lead for further development. Following this, and mostly parallel to the work presented here, rational drug design led to the development of related derivatives containing various $O$-linked substituents on the naphthyl ring condensed with the flavonoid skeleton. Several of these compounds, and particularly one containing an $O$-tetrahydrogeranyl moiety, were designed and confirmed as both strong cytotoxic agents and potent inhibitors of glutathione S-transferase $\Omega 1$ [55] [56].

Attempting to find the minimum pharmacophore of protoflavonoids, another research group performed the total synthesis of an analog (RY10-4) consisting of the B and C ring of protoapigenone. Similarly to the above-mentioned synthetic efforts, the six steps procedure was finished with an oxidative de-aromatization catalyzed by PIFA in a solvent mixture of acetonitrile and water. Based on their results, the authors concluded that the flavonoid A-ring is not necessary for the anticancer effects of protoflavones. An overview of the synthetic analogs of protoflavonoids is presented in Fig. 3.
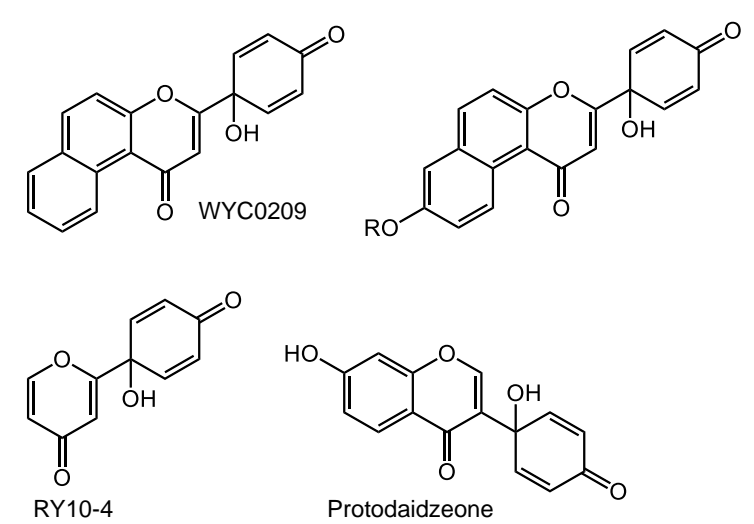

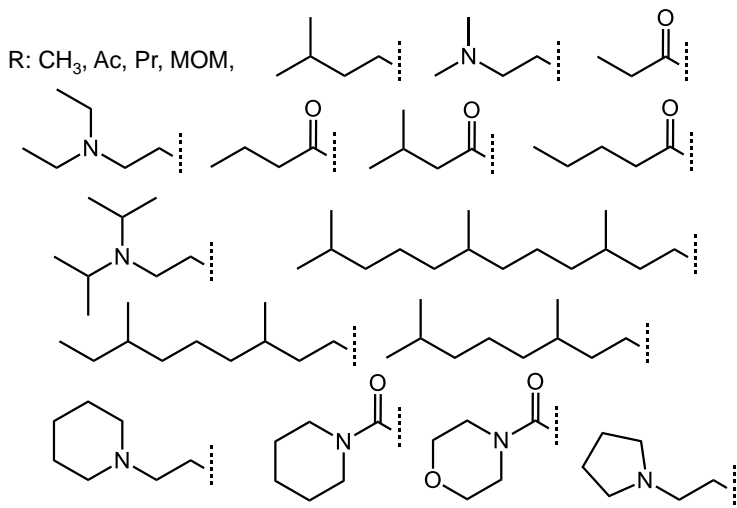

Figure 3. Structures of synthetic protoflavonoid analogs prepared by others 


\subsection{Direct preliminaries of the $\mathrm{PhD}$ work}

As a direct antecedent of the work described here, an optimization of the critical step of the total synthesis, namely the oxidative de-aromatization of the B-ring was attempted by A. Hunyadi, supervisor of my doctoral studies. During this work, it has been revealed that a semisynthetic preparation of protoapigenone (1) is also possible directly from apigenin [42], by using PIFA as oxidant and by keeping the concentration of apigenin at a maximum of $1 \mathrm{mg} / \mathrm{mL}$ in acetonitrile - water $(9: 1, \mathrm{v} / \mathrm{v})$. This finding allowed a fast and economic synthesis of $\mathbf{1}$ up to the gram scale, representing a breakthrough in studying the bioactivity of this interesting flavonoid. Moreover, with the preparation of a set of 1'-alkoxyprotoflavones (Fig. 4), important new structure-activity relationships were also revealed. Previously, the presence of a symmetric and non-substituted $p$-quinol B-ring was thought to be an absolute need for a strong proapoptotic activity. Indeed, neither of the partly or completely saturated or the 4'-hydroxy compounds, nor the 1'-methoxylated synthetic analogs were found before as potent in this regard as protoapigenone (1) [28]; [38]; [20] ; [18]; [33]. In contrast with this, however, when a longer, non-branching aliphatic side-chain (n-propyl or $n$-butyl) was introduced at C-1' instead of a methoxy group, an equivalent or even increased cytotoxic activity was found as compared to that of $\mathbf{1}$ [42].
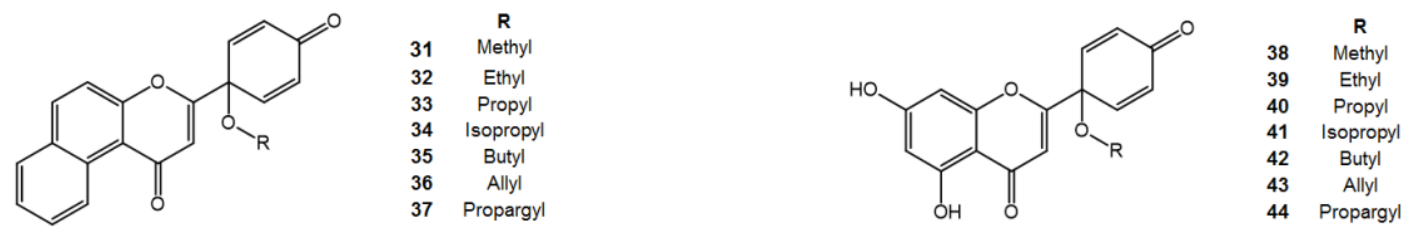

Figure 4. 1-O'-alkylprotoflavones prepared as direct preliminaries of this work

At the beginning of my $\mathrm{PhD}$ studies I had the chance to be partially involved in this work, which then served as the head-start for the studies described hereinafter. 


\section{Objectives}

As per the above, the following objectives were set up for this work.

1. As a primary aim of the work, to prepare new A-ring modified synthetic protoflavones and their 1'-O-alkoxy derivatives.

2. To perform an in vitro and in silico study on the potential formation of protoapigenone (1) upon ROS scavenging by apigenin.

3. To test the cytotoxicity of the newly prepared compounds and to extend related structureactivity relationships, with a strong focus on multi-drug resistant cancer.

4. To search for other potential bioactivities of these compounds, which are not related to the cytotoxic effect.

\section{Materials and methods}

\subsection{Reagents and starting materials}

Commercially available 4'-hydroxyflavones (4'-hydroxy-6-methylflavone, 4'-hydroxy-6methoxyflavone, 4'-hydroxy- $\beta$-naphthoflavone) were purchased from Indofine Chemical Company, Inc. (Hillsborough USA). Chemicals were obtained from Aldrich, Inc. (USA). Solvents of HPLC grade were from Merck (Darmstadt, Germany). Solvents of analytical grade were obtained from Molar (Halásztelek, Hungary).

\subsection{General experimental procedures}

\subsubsection{Synthesis}

Synthesis of 5 '-ethyl- and 5 '-pentyl-2'-hydroxyacetophenone (48 and 49). In the first step, 4-ethyl- and 4-pentylphenol acetate (46 and $\mathbf{4 7}$, respectively) were synthesized by adding 0.1 mol (10.2 g) of acetic anhydride and one drop of $\mathrm{ccH}_{2} \mathrm{SO}_{4}$ to $0.1 \mathrm{~mol}$ of 4-ethyl or 4pentylphenol and stirring at room temperature for $20 \mathrm{~min}$. The mixture was then poured into water and extracted with $3 \times 50 \mathrm{ml}$ EtOAc. The organic layer was evaporated under reduced pressure, re-dissolved in $\mathrm{CH}_{2} \mathrm{Cl}_{2}$ and crystallized anhydrous $\mathrm{AlCl}_{3}$ was added little by little under ice bed cooling. The mixture was refluxed for $10 \mathrm{~h}$, then the reaction was stopped by adding crushed ice. After filtration, the precipitate was purified on silica to obtain (48) or (49).

General Procedure for chalchone synthesis. The 5'-substituted 2 '-hydroxyacetophenone (4851) and 4-benzyloxybenzaldehyde ( $3.0 \mathrm{~g}, 14.3 \mathrm{mmol}$ ) were dissolved in 50\% EtOH, $\mathrm{KOH} / \mathrm{H}_{2} \mathrm{O}$ 
solution $(20 \mathrm{~mL})$. The reaction was stirred at r.t. for $30 \mathrm{~h}$, and then the solvent was evaporated under reduced pressure. The mixture was purified on silica gel (isocratic elution, nhexane/EtOAc, 6:1) to afford (52-55), respectively.

General Procedure for Flavone synthesis. $3 \mathrm{mmol}$ of chalchone (52-55) was dissolved in DMSO $(5 \mathrm{~mL})$, and 1 eq. iodine $(76 \mathrm{mg}, 0.3 \mathrm{mmol})$ was added. The solution mixture was stirred and heated to $110{ }^{\circ} \mathrm{C}$. After $2 \mathrm{~h}, 10 \% \mathrm{Na}_{2} \mathrm{~S}_{2} \mathrm{O}_{3}(50 \mathrm{~mL})$ was added to remove iodine. The mixture was extracted with EtOAc (3 X $50 \mathrm{~mL})$ and then purified by column chromatography on silica gel to afford compounds 49-52, respectively.

Synthesis of compound (53) from (52) via Suzuki-coupling. Compound (52) (407.26 mg, 1.0 mmol), 1.2 eq. phenylboronic acid (146.3 mg, $1.2 \mathrm{mmol}$ ) and 2 eq. (276.42mg, $2.0 \mathrm{mmol}$ ) $\mathrm{K}_{2} \mathrm{CO}_{3}$ were dissolved in $7 \mathrm{~mL}$ of water, and $25 \mathrm{~mL}$ of propanol and $5 \mathrm{mmol}$ of Tetrakis(triphenylphosphine)palladium(0) were subsequently added. The reaction was performed under $\mathrm{N}_{2}$ gas at $40^{\circ} \mathrm{C}$, to afford $(\mathbf{5 3})$ as a yellow solid.

General Procedure for Benzyl Group Removal. A mixture of $0.5 \mathrm{mmol}$ of the 4'benzyloxyflavone (49-53), dry $\mathrm{Pd} / \mathrm{C}(10 \%, 106 \mathrm{mg})$, and $20 \mathrm{~mL}$ of EtOAc was stirred at $25^{\circ} \mathrm{C}$ under an atmosphere of hydrogen for $10 \mathrm{~h}$. The mixture was filtered, washed with EtOAc, concentrated under vacuum and purified by column chromatography on silica gel to afford compounds (54-58), respectively.

General procedure for protoflavone synthesis from 4'-hydroxyflavones. Apigenin, genkwanin, 4'-hydroxy-6-methylflavone, 4'-hydroxy-6-methoxyflavone, 4 '-hydroxy- $\beta$ naphthoflavone (Indofine, Hillsborough, NJ, USA), or the synthesized 4'-hydroxyflavone (6165) was dissolved at $1 \mathrm{mg} / \mathrm{mL}$ concentration in a 9:1 v/v ratio mixture of acetonitrile and water or the alcohol to be coupled at position C-1'. Two equivalents of [bis(trifluoroacetoxy)iodo]benzene were added to the mixture. After stirring at $80^{\circ} \mathrm{C}$ for 1 hour, the mixture was cooled down, evaporated under reduced pressure and purified by flash chromatography to obtain the pure compounds . 


\subsubsection{Chromatographic techniques}

\section{Thin-layer chromatography (TLC)}

Normal-phase (NP) TLC analyses were performed on Silica gel $60 \mathrm{~F}_{254}$ and reversed-phase (RP) TLC on Silica gel 60 RP-18 F $_{254}$ S (E. Merck, Darmstadt, Germany) plates. The plates were developed by an ascending technique in a glass chamber (Desaga, Heidelberg, Germany) at room temperature. The following NP- and RP-TLC solvent systems were utilized: NP-TLC ${ }_{1}$ : hexane-ethyl acetate (90:10, v/v); NP-TLC 2 : hexane-ethyl acetate (70:30, v/v); NP-TLC 3 : hexane-ethyl acetate $(50: 50, \mathrm{v} / \mathrm{v})$; NP-TLC 4 : hexane-ethyl acetate $(30: 70, \mathrm{v} / \mathrm{v}) ;$ RP-TLC $_{1}$ : acetonitrile-water $(35: 65, \mathrm{v} / \mathrm{v})$; RP-TLC 2 : methanol-water $(60: 40, \mathrm{v} / \mathrm{v}) ; \mathrm{RP}^{-T_{2}} \mathrm{CL}_{3}:$ methanol- $^{-}$ water (30:60, v/v); RP-TLC4: tetrahydrofuran-water (50:50, v/v).

\section{Rotation Planar chromatography (RPC)}

Compounds were purified by rotation planar chromatography on a Chromatothron equipment (Harrison Research, USA). The stationary phase for RPC was silica gel $60 \mathrm{GF}_{254}$. The following mobile phases were utilized.

$\mathrm{RPC}_{1}$ : n-hexane/ethyl acetate $(11: 3, \mathrm{v} / \mathrm{v}) ; \mathrm{RPC}_{2}$ : n-hexane/ethyl acetat $(9: 1, \mathrm{v} / \mathrm{v}) ; \mathrm{RPC}_{3}: \mathrm{n}-$ hexane/ethyl acetat $(8: 2, \mathrm{v} / \mathrm{v}) ; \mathrm{RPC}_{4}:$ n-hexane/ethyl acetat $(6: 3, \mathrm{v} / \mathrm{v}) ; \mathrm{RPC}_{5}$ : chloroformmethanol $(97: 3, \mathrm{v} / \mathrm{v})$.

\section{High-perfomance liquid chromatography (HPLC)}

HPLC analyses were performed by a gradient HPLC system of two Jasco PU-2080 pumps coupled to a Jasco MD-2010 Plus diode-array detector (DAD) controlled by the ChromNAV chromatographic software. The following normal- (NP) and reverse-phase (RP) HPLC columns were utilized:

Analytical - NP-HPLC 1 : Zorbax-SIL column (5 $\mu \mathrm{m}, 250$ x $4.6 \mathrm{~mm})$ (DuPont, Paris, France); RP-HPLC ${ }_{1}$ : Zorbax SB C18 (5 $\left.\mu \mathrm{m}, 250 \times 4.6 \mathrm{~mm}\right)$ (DuPont, Paris, France); Semi-preparative

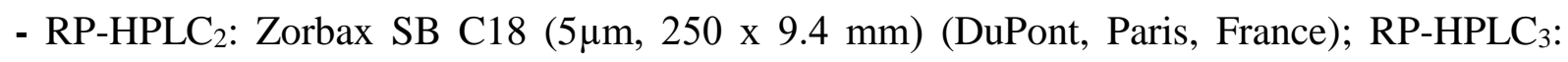

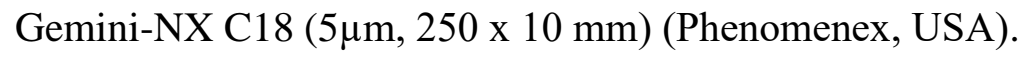




\section{Flash chromatography}

Flash Chromatography (FC) was performed on a Combiflash Rf+ equipment (TELEDYNE Isco, USA) on RediSep normal-phase $24 \mathrm{~g}$ silica disposable flash columns (40-60 $\mu \mathrm{m}$; Teledyne Isco, USA). The following solvent systems were utilized:

NP-FC 1 : Gradient system was $0 \%$ Ethyl acetate gradually increasing to $100 \%$ in 30 min at a flow rate of $30 \mathrm{~mL} / \mathrm{min} ; \mathrm{NP}-\mathrm{FC}_{2}$ : Gradient system was $0 \%$ Methanol gradually increasing to $5 \%$ in $25 \mathrm{~min}$ at flow rate of $30 \mathrm{~mL} / \mathrm{min}$.

\section{Centrifugal Partition Chromatography (CPC)}

Centrifugal Partition Chromatography were carried on an Armen SCPC-250-F equipment (Armen Instrument, France). The following biphasic solvent systems were utilized:

$\mathrm{CPC}_{1}$ : hexane-ethyl acetate-methanol-water $(7: 3: 7: 3, \mathrm{v} / \mathrm{v} / \mathrm{v} / \mathrm{v}) ; \mathrm{CPC}_{2}$ : hexane-ethyl acetatemethanol-water $(6: 4: 6: 4, \mathrm{v} / \mathrm{v} / \mathrm{v} / \mathrm{v})$

\section{Capillary zone electrophoresis (CE)}

Aiming to study the apigenin-protoapigenone transformation during the Fenton reaction, capillary zone electrophoresis was performed in collaboration. An Agilent Capillary Electrophoresis ${ }^{3 \mathrm{D}} \mathrm{CE}$ system (Agilent Technologies, Waldbronn, Germany) was used, applying bare fused silica capillary having a $64.5 \mathrm{~cm}$ total and $56 \mathrm{~cm}$ effective length with $50 \mu \mathrm{m}$ I.D, and with a bubble cell detection window (Agilent Technologies, Santa Clara, CA, USA). Online absorption at $230 \mathrm{~nm}$ and $340 \mathrm{~nm}$ was monitored by DAD UV-Vis detector. The capillary thermostat was set for $25^{\circ} \mathrm{C}$. Between measurements, the capillary was rinsed subsequently with $0.1 \mathrm{M} \mathrm{HCl}, 1.0 \mathrm{M} \mathrm{NaOH}, 0.1 \mathrm{M} \mathrm{NaOH}$ and distilled water for 3 minutes each, and with BGE ( $50 \mathrm{mM}$ sodium tetraborate $\left(\mathrm{pH} 9.3\right.$ ) for 5 minutes. Samples were injected by $5 \times 10^{3} \mathrm{~Pa}$ pressure for $6 \mathrm{sec}$. Runs were performed in the positive-polarity mode with $27 \mathrm{kV}$.

\subsubsection{Structure elucidation}

Structure elucidation was carried out by means of nuclear magnetic resonance (NMR) spectroscopy and mass spectroscopy (MS). NMR spectra were obtained on a Varian Gemini2000200 MHz or Bruker Avance DRX-500 NMR spectrometer in chloroform- $d_{1}$, methanol$d_{4}$, acetone- $d_{6}$, or dimethyl sulfoxide- $d_{6}$. Mass spectra were taken on an API 2000 triplequadrupole (Ab Sciex, USA) or LCMS-IT-TOF (Shimadzu, Japan) with and ESI interface. 


\subsubsection{In silico studies on the formation of protoapigenone from apigenin}

Calculations were achieved in the Gaussian09 software within the DFT (Density Functional Theory) formalism. Results were obtained on Intel Xeon Sandy Bridge E5-2620 (CAlcul en LImousin, Université de Limoges, Limoges, France). The B3P86 functional was chosen as it has shown a very good agreement between theoretical and experimental values of bond dissociation enthalpies (BDE). For the phenolic group O-H bond dissociation enthalpies (BDE), were calculated as the difference between $\mathrm{Fl}-\mathrm{OH}$ (the flavonoid) and $\mathrm{Fl}-\mathrm{O}^{\circ}+\mathrm{H}^{\cdot}$ (the corresponding radicals formed after $\mathrm{H}$-atom abstraction (HAT) from $\mathrm{Fl}-\mathrm{OH}$ to the free radical. The effect of solvent was taken into account by using the integral-equation-formalism polarizable continuum model (IEF-PCM).

\subsubsection{Experimental studies on the apigenin-protoapigenone transformation}

$10 \mathrm{mg}$ of apigenin was dissolved in $10 \mathrm{ml} \mathrm{50 \%}$ aqueous $\mathrm{MeOH}$, and the $\mathrm{pH}$ was adjusted to $\mathrm{pH}=4$ by using $\mathrm{H}_{2} \mathrm{SO}_{4} .1 .02 \mathrm{mg}$ iron catalyst $\left(\mathrm{FeSO}_{4} \cdot 7 \mathrm{H}_{2} \mathrm{O}\right)$ was added, followed by the slow addition of $0.11 \mathrm{ml}$ of $30 \% \mathrm{H}_{2} \mathrm{O}_{2}$. After 60 minutes the reaction mixture was purified by using SPE on C18 stationary phase, and investigated by HPLC.

\subsubsection{Bioassays}

\section{Cell lines.}

L5178 mouse T-cell lymphoma cell line (ECACC catalog no. 87111908, U.S. FDA, Silver Spring, MD, U.S.), and its sub-cell line L5178 ${ }_{\mathrm{B} 1}$, derived from L5178 by transfection with $\mathrm{pHa}$ MDR1/A retrovirus (57), were cultured in McCoy's 5A media supplemented inactivated horse serum. L5178 1 B1 cell line was selected by culturing the infected cells with $60 \mu \mathrm{g} / \mathrm{L}$ colchicine (Sigma). Breast cancer cell lines MCF7 and its sub-cell line obtained by adaptation to doxorubicin, MCF7 $7_{\text {Dox }}$ (58) were cultured in EMEM media supplemented with non-essential amino acids, $1 \mathrm{mM}$ Na-pyruvate and 10\% inactivated fetal bovine serum (MCF7 Dox was cultured in presence of $1 \mu \mathrm{M}$ of doxorubicin each third passage). All cells were cultured at $37^{\circ} \mathrm{C}$ and $5 \%$ $\mathrm{CO}_{2}$; all media contained Nystatin, $2 \mathrm{mM}$ of L-glutamine, $100 \mathrm{U}$ of penicillin and $0.1 \mathrm{mg}$ of streptomycin, purchased from Sigma. MES-SA human uterine sarcoma cell line and the doxorubicin selected MES-SA/Dx5 were obtained from ATCC. The human cervix carcinoma cell line KB-3-1 and its vinblastine selected derivative KB-V1 were a kind gift from Dr. Michael M. Gottesman (National Institutes of Health). A431 and the retrovirally transduced $\mathrm{A} 431_{\mathrm{B} 1}$ and $\mathrm{A} 431_{\mathrm{G} 2}$ are human skin-derived, epidermoid carcinoma cells were kind gifts from Dr. K. Német. MES-SA, KB-3-1, A431 and their derivative cell lines were maintained in 
DMEM completed with 10\% FBS, $5 \mathrm{mM}$ glutamine and 50 units $/ \mathrm{mL}$ penicillin and streptomycin (Life Technologies).

\section{Cytotoxicity assays.}

In case of mouse lymphoma cell lines, $2 \times 10^{4}$ cells per well were cultured in 96-wells microplates with different concentrations of the tested compound, in McCoy's 5A media, at $37^{\circ} \mathrm{C}$ and $5 \% \mathrm{CO}_{2}$, for $24 \mathrm{~h}$. With respect to the MCF-7 and MCF-7 $7_{\text {Dox }}, 1 \times 10^{4}$ cells per well were seeded overnight and serial dilutions of the compounds were added the following day and incubated for $48 \mathrm{~h}$. In all cases, after the incubation time, $10 \%$ MTT was added to each well and incubated for $4 \mathrm{~h}$, when SDS was added to the medium. Results were read after o/n incubation. Fifty per cent inhibitory concentrations $\left(\mathrm{IC}_{50}\right)$ were calculated using nonlinear regression curve fitting of $\log$ (inhibitor) versus normalized response with a variable slope and least squares (ordinary) fit of GraphPad Prism 5 software, for three independent samples.

In the case of A431 cell lines, MES-SA cell lines, KB-3-1 and KB-V1 cell lines, $5 \times 10^{3}$ cells per well were cultured and incubated overnight in 96-well microplates. Serially diluted drugs were then added, and plates were incubated for additional 72 hours. Cytotoxicity was measured by Presto Blue cell viability reagent (Invitrogen) in a final dilution of $5 \%$.

\section{FACS measurements}

Calcein-AM uptake assay was performed as described earlier (59). Briefly, 250.000 cells per tube were pre-incubated for 5 minutes in the presence of $50 \mu \mathrm{M}$ verapamil or the test protoflavone compounds. Calcein-AM was added at a final concentration of $250 \mathrm{nM}$ and incubated for an additional 10 minutes. Samples were then washed with ice-cold PBS and were kept on ice until measured by an Attune ${ }^{\circledR}$ Acoustic Focusing Cytometer. The mitoxantrone uptake assay was carried out similarly: tariquidar $(1 \mu \mathrm{M})$ was added to the cells for 5 minutes at 37C (pre-incubation), and Mitoxantrone was added at $4 \mu \mathrm{M}$ for an additional 20 minutes at 37C. The reaction was stopped by adding serum-free DMEM; the samples were immediately measured by FACS.

\section{Xanthine oxidase inhibition assay and determination of the binding mode of (44)}

XO inhibition activity for compounds (31-44) and related data for the dose-effect curves for (44), apigenin and allopurinol, were obtained by using commercially available XO activity assay kit (Sigma-Aldrich Ltd., USA), following the provided protocol. 
For determination of the binding mode of (44), the following procedure was applied. The activity of XO was determined by measuring the production of uric acid, at $290 \mathrm{~nm}$ for 3 min in 96-well plates, using a FluoSTAR OPTIMA plate reader (BMG LABTECH). The protocol was adapted from that recommended by Sigma-Aldrich for a total volume of the assay of $300 \mu \mathrm{L}$. Stock solutions were prepared as follows: $50 \mathrm{mM}$ potassium buffer, $\mathrm{pH}$ 7.5; $0.9 \mathrm{mM}$ $\mathrm{XO}, \mathrm{pH} 7.5 ; 1.5,0.6,0.3,0.15$ and $0.075 \mathrm{mM}$ solutions of (44) in DMSO; and 0.04 units/mL XO enzyme. From the $0.9 \mathrm{mM}$ xanthine solution further stock solutions were prepared with concentrations between 7.5 and $600 \mu \mathrm{M}$. A $140 \mu \mathrm{L}$ buffer solution mixed with a $100 \mu \mathrm{L}$ xanthine solution, at the proper concentration, was added to the wells at final concentrations of $33 \mathrm{mM}$ (buffer) and 2.5-300 $\mu \mathrm{M}$ (xanthine). A $10 \mu \mathrm{L}$ solution of (44) was added to the respective wells, with final concentrations of 2.5, 5, 10 and $20 \mu \mathrm{M}$. The final concentration of DMSO in the assay did not exceed $3.3 \%$ of the total volume. The reaction was initiated by automatic addition of $50 \mu \mathrm{L}$ XO solution (final concentration of 0.006 units $/ \mathrm{mL}$ ). Allopurinol was used as a positive control. For each concentration of xanthine, the four concentrations of (44) were tested in five replicates.

\section{Docking study on protoapigenone 1 '- $O$-propargyl ether to the active center of xanthine oxidase}

The 3D structure of (44) was optimized prior to docking, by the Gaussian09 (Gaussian Inc., Wallingford, USA) software. The DFT (Density Functional Theory) formalism was used with the hybrid B3P86 functional that has been shown accurate enough to assess geometrical and energetic features of flavonoids. The $6-31+G(d, p)$ basis set was sufficient to provide a relevant geometry. The optimized structure was subsequently used for the docking study.

The 3NVY structure, representing 3D structure of bovine $\mathrm{XO}$ in complex with quercetin, was downloaded from www.pdb.org, and used without modification. Docking study was performed by using iGEMDOCK 2.1 (BioXGEM, Hsinchu, Taiwan) at default settings for "accurate docking"; population size: 800 , generations: 80 , number of solutions: 10 . Ligand binding site was defined with a $15 \AA$ radius around the X-ray position of quercetin. Scoring function was left at its default settings with equal preferences to hydrophobic and electrostatic interactions. Docking was validated by re-docking the "Que" residue into the macromolecule in mol2 format in order to allow flexible docking. RMSD calculations, as well as visualization of the ligandresidue interactions were achieved with Discovery Studio 3. 


\section{Results}

\subsection{Synthesis}

Thirty-seven protoflavones and protoflavone 1'-O-alkyl ethers were synthesized from commercially available 4'-hydroxyflavones (apigenin, genkwanin, 4'-hydroxy-6methylflavone, 4 '-hydroxy-6-methoxyflavone and 4 '-hydroxy- $\beta$-naphthoflavone) by utilizing PIFA-mediated oxidative de-aromatization. 20 of these protoflavone derivatives were prepared as new compounds (60) [I,II,VII].

Scheme 2. Synthesis of protoflavones from commercially available $4^{\prime}$-hydroxyflavones ${ }^{a}$<smiles>[R]c1cc2oc(-c3ccc(O)cc3)cc(=O)c2c([R])c1[R]</smiles>

$\mathrm{R}^{1}=\mathrm{OMe}, \mathrm{R}^{2}=\mathrm{H}, \mathrm{R}^{3}=\mathrm{OH}$

81: $\mathrm{R}=\mathrm{H}$

82: $\mathrm{R}=\mathrm{Me}$

83: $R=E t$

84: $R=$ Pro

85: $R=$ But

86: $R=A l l y \mid$

87: $R=$ Propargyl

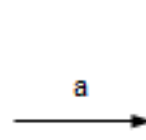

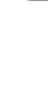

$R^{1}=H, R^{2}=M e, R^{3}=H$

88: $\mathrm{R}=\mathrm{H}$

89: $\mathrm{R}=\mathrm{Me}$

90: $R=E t$

91: $R=P r o$

92: $\mathrm{R}=\mathrm{But}$

93: $\mathrm{R}=\mathrm{Ally|}$

94: $R=$ Propargyl<smiles>[R]c1cc2oc(C3([R])C=CC(=O)C=C3)cc(=O)c2c([R])c1[R]</smiles>

$\mathrm{R}^{1}=\mathrm{H}, \mathrm{R}^{2}=\mathrm{OMe}, \mathrm{R}^{3}=\mathrm{H}$

95: $\mathrm{R}=\mathrm{H}$

96: $R=M e$

97: $\mathrm{R}=\mathrm{Et}$

98: $\mathrm{R}=\mathrm{But}$

99: $\mathrm{R}=\mathrm{Allyl}$

100: R=Propargyl<smiles>O=c1cc(-c2ccc(O)cc2)oc2ccc3ccccc3c12</smiles>

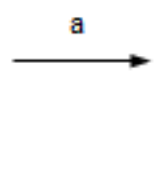

$\mathrm{R}^{1}=\mathrm{OH}, \mathrm{R}^{2}=\mathrm{H}, \mathrm{R}^{3}=\mathrm{OH}$

1: $\mathrm{R}=\mathrm{H}$

38: $\mathrm{R}=\mathrm{Me}$

39: $R=E t$

40: $R=$ Pro

41: $\mathrm{R}=i-$ Pro

42: $\mathrm{R}=$ But

43: $\mathrm{R}=\mathrm{Allyl}$

44: $R=$ Propargyl

45: $\mathrm{R}=\mathrm{Bn}$

WYC0209: $\mathrm{R}=\mathrm{H}$

31: $R=M e$

32: $R=E t$

33: $\mathrm{R}=$ Pro

34: $\mathrm{R}=i-$ Pro

35: $R=B u t$

36: $R=A l y \mid$

37: R=Propargyl

${ }^{a}$ Reagents: a) $\mathrm{CH}_{3} \mathrm{CN} / \mathrm{ROH}$ 9/1, PIFA (2 eq).

Total synthesis of a set of various 6-substituted protoflavones was achieved in 4-6 steps [VII]. In order to obtain starting materials (i.e. $5^{\prime}$-ethyl-2'-hydroxyacetophenone, (45); and 5' -pentyl2'-hydroxyacetophenone; (42) for our 6-ethyl and 6-pentyl substituted target compounds, the appropriate $p$-substituted phenols were acetylated and subjected to Fries-rearrangement reaction under the condition of dry $\mathrm{AlCl}_{3}$ in dichloromethane (61). The resulting 2'hydroxyacetophenones and those commercially available with a 5 '-ethoxy or -bromo substituent ((49) and (50), respectively) were utilized in Claisen-Schmidt condensation reactions with p-benzyloxybenzaldehyde to yield chalchones (51-54), which, after performing ring closure with iodine in DMSO, yielded the corresponding 6-substituted 4'benzyloxyflavones (49-50). The 6-bromo substituted compound (58) was subjected to Suzuki coupling in order to obtain the corresponding 6-phenylflavone (59). Debenzylation of the 
flavonoids obtained this way and subsequent oxidative de-aromatization of the flavones (6064) with PIFA, as described above, allowed us to obtain the protoflavones with various substituents at positions C-6 and C-1' (65-79). 9 of the protoflavone derivatives were obtained as new compounds. Scheme 3 summarizes the total synthetic procedure.

Scheme 3. Total synthesis of 6-substituted protoflavone derivatives ${ }^{a}$

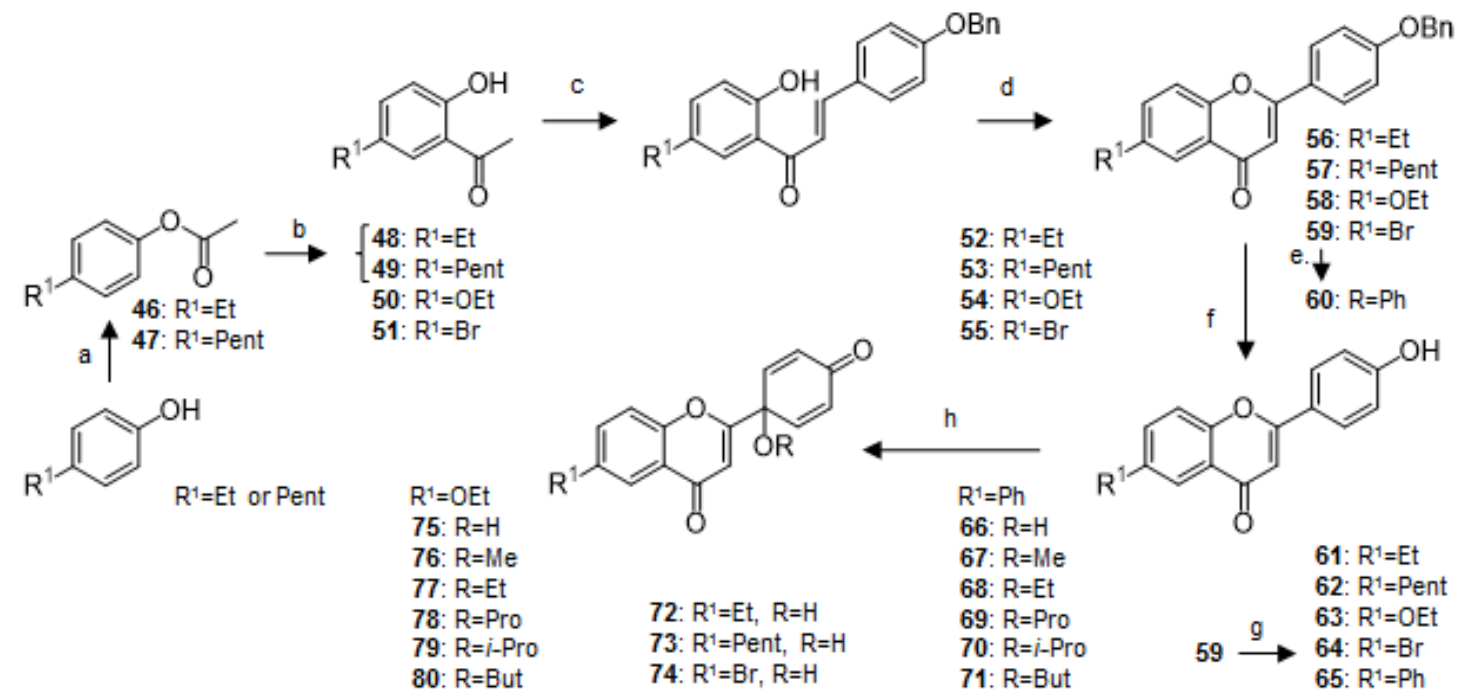

${ }^{a}$ Reagents: a) $\left(\mathrm{CH}_{3} \mathrm{CO}\right)_{2} \mathrm{O}, c c \mathrm{H}_{2} \mathrm{SO}_{4}$; b) $\mathrm{AlCl}_{3}$; c) EtOH, 4-benzyloxybenzaldehyde, 50\% $\mathrm{KOH} / \mathrm{H}_{2} \mathrm{O}$; d) $\mathrm{I}_{2}$, DMSO; e) Phenylboronic acid, $\mathrm{K}_{2} \mathrm{CO}_{3}$, Tetrakis(triphenylphosphine)palladium(0); f) $10 \% \quad \mathrm{Pd}-\mathrm{C} / \mathrm{H}_{2} ; \mathrm{g}$ ) TFA/ $\mathrm{CH}_{2} \mathrm{Cl}_{2}$, reflux; h) $\mathrm{CH}_{3} \mathrm{CN} / \mathrm{ROH}$ 9/1, PIFA (2 eq).

\subsection{The role of $\mathrm{OH}$ radical scavenging in the formation of $\mathbf{1}$ from apigenin}

The possible formation of protoapigenone (1) from apigenin was first studied in silico, within the DFT (Density Functional Theory) formalism. Bond dissociation enthalpies (BDEs) for the 4'-OH group of apigenin were calculated in the gas phase or by taking into account the effect of solvent (see Materials and Methods, page 15). Electron spin density was calculated for the resulting phenoxyl radical in order to have a rough estimation on the likelihood for the position where an $\mathrm{OH}$ radical could possibly attack this intermediate. Results of these calculations, as well as those of the thermodynamic analysis are summarized in Scheme 4 and Figure 5.

Scheme 4. Possible formation of protoapigenone from apigenin upon ${ }^{\circ} \mathrm{OH}$ radical scavenging. BDE: bond dissociation enthalpy in vacuum or in solution.

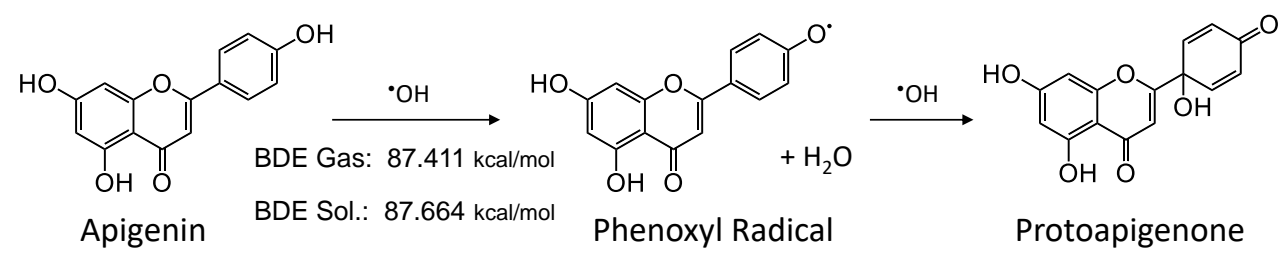


A

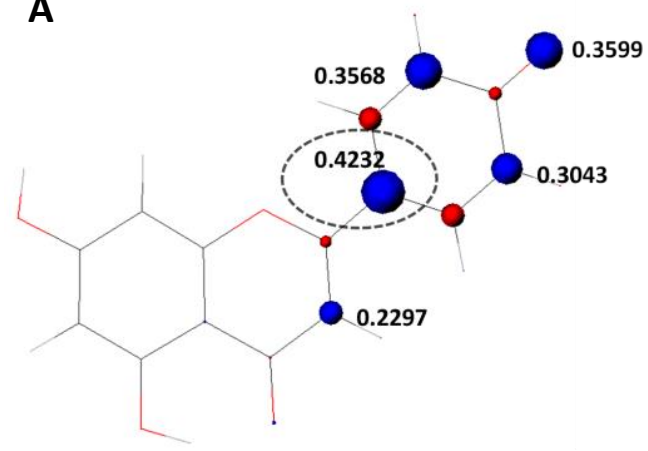

B

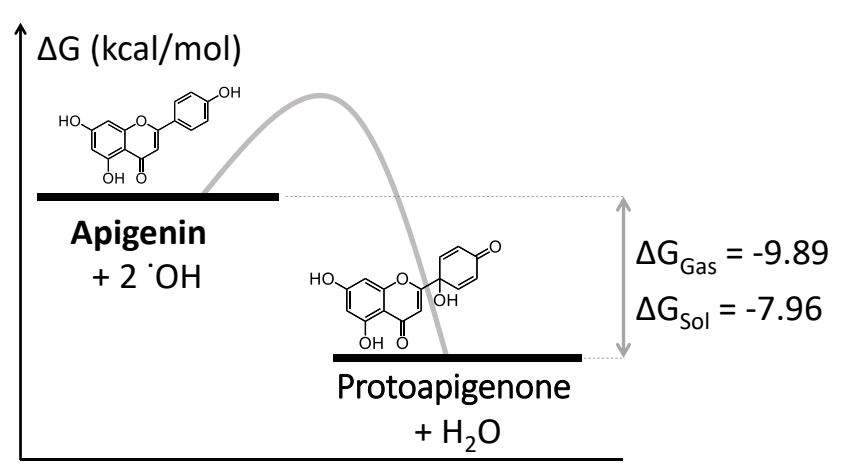

Figure 5. A: Spin density map of the 4'-phenoxyl radical of apigenin, indicating that the favorable position for the radical is situated at $\mathrm{C}-1^{\prime}$ that makes this carbon a potential target for further scavenging. B: Thermodynamic analysis of the whole process of forming protoapigenone from apigenin through $\mathrm{OH}$ radical scavenging.

Based on the above in silico results, it can be stated that, even though the initiating hydrogen atom transfer requires a relatively large energy, such a transformation is indeed possible. In order to obtain experimental verification or disproof to this hypothesis, Fenton's reaction was performed on apigenin, and the resulting mixture was analyzed by RP-HPLC-DAD after prepurification, or CE-UV with a direct injection. The same reaction was also performed on protoapigenone, in order to investigate its possible further decomposition within the reaction mixture. Furthermore, the possible reduction of protoapigenone to apigenin was studied by incubating it with reduced glutathione (GSH) for 24h. Fig. 6 presents the results of these studies.
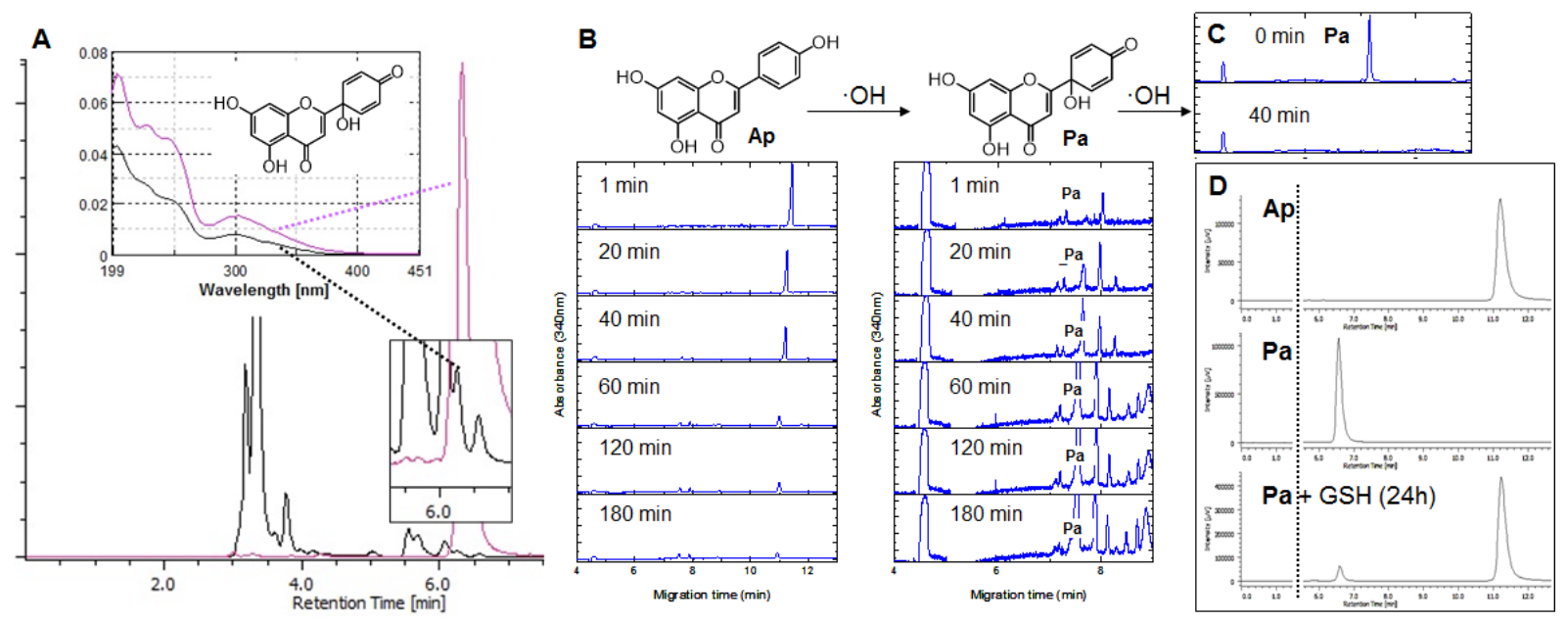

Figure 6. Studies on the apigenin-protoapigenone-apigenin redox transformation. Ap: apigenin, Pa: protoapigenone (1). A: HPLC-DAD fingerprint on the Fenton reaction mixture of Ap (black) in comparison with that of $\mathbf{1}$ (purple). B: CE studies on the decomposition of Ap and the formation of $\mathbf{1}$ over the time of a Fenton reaction. $\mathbf{C}$ : CE study on the decomposition of $\mathbf{1}$ in a Fenton reaction. D: HPLC chromatograms of $\mathbf{1}$ before and after a $24 \mathrm{~h}$ incubation with GSH in comparison with that of Ap. 
As seen from the above figure, the formation of $\mathbf{1}$ from apigenin does take place in the presence of excess amounts of $\mathrm{OH}$ radicals (i.e. Fenton reaction). Moreover, despite its much faster decomposition (complete disappearance after $40 \mathrm{~min}$; see Fig 6C) as compared to that of apigenin (still detectable after $180 \mathrm{~min}$; see Fig 6B, left panel), $\mathbf{1}$ is continuously present over the reaction (see Fig 6B, right panel). This strongly suggests that $\mathbf{1}$ is in fact a major primary product forming when apigenin scavenges $\mathrm{OH}$ radicals.

\subsection{Anticancer activity}

The cytotoxicity of compounds (Gen, 6Me) were tested in collaboration with the team of Prof. Dr. Fang-Rong Chang (Graduate Institute of Natural Products, Kaohsiung Medical University, Kaohsiung, Taiwan) on HepG2, Hep3B, A549 and MDA-MB-231 human cancer cell lines [II]; results are summarized in Table 2.

Table 2. The cytotoxicity of protoflavones 81-94 on HepG2, Hep3B, A549 and MDA-MB-231 human cancer cell lines. Intervals indicate $95 \%$ confidence intervals of the $\mathrm{IC}_{50}$ values as calculated by GraphPad Prism 5.0; n=3-4; Dox=doxorubicin.

\begin{tabular}{|c|c|c|c|c|}
\hline & \multicolumn{4}{|c|}{$\mathrm{IC}_{50}(\mu \mathrm{M})$} \\
\hline Compound & HepG2 & Hep3B & A549 & MDA-MB-231 \\
\hline Pa & $\mathbf{3 . 0 7}(2.83-3.34)$ & $\mathbf{1 . 2 1}(1.19-1.24)$ & $\mathbf{1 1 . 2 9}(10.07-12.65)$ & $\mathbf{1 . 3 5}(1.17-1.55)$ \\
\hline WYC0209 & $\mathbf{1 . 0 6}(1.04-1.08)$ & $\mathbf{0 . 2 5}(0.20-0.30)$ & $\mathbf{2 . 1 9}(2.03-2.37)$ & $\mathbf{0 . 4 3}(0.41-0.45)$ \\
\hline 6-OMe & $\mathbf{1 . 8 6}(1.85-1.88)$ & $\mathbf{0 . 6 4}(0.55-0.73)$ & $\mathbf{1 . 7 7}(1.50-2.08)$ & $\mathbf{0 . 7 0}(0.64-0.77)$ \\
\hline $\mathbf{6 - O M e}$ & $\mathbf{7 . 6 5}(7.44-7.87)$ & $\mathbf{1 . 8 8}(1.55-2.28)$ & $\mathbf{1 2 . 0 9}(8.95-16.34)$ & $\mathbf{2 . 0 8}(1.96-2.22)$ \\
\hline $\mathbf{6 - O M e}$ & $\mathbf{7 . 2 7}(7.05-7.50)$ & $\mathbf{1 . 8 2}(1.73-1.91)$ & $\mathbf{1 0 . 9 7}(9.24-13.03)$ & $\mathbf{2 . 0 9}(1.99-2.20)$ \\
\hline 6-OMe & $\mathbf{8 . 6 9}(8.05-9.38)$ & $\mathbf{1 . 8 3}(1.33-2.52)$ & $\mathbf{1 0 . 2 7}(9.49-11.11)$ & $\mathbf{2 . 2 0}(2.05-2.36)$ \\
\hline $\mathbf{6 - O M e}$ & $>\mathbf{5 0}$ & $>\mathbf{5 0}$ & $>\mathbf{5 0}$ & $>\mathbf{5 0}$ \\
\hline 6-OMe & $\mathbf{1 2 . 2 7}(11.70-12.87)$ & $\mathbf{0 . 6 5}(0.61-0.68)$ & $\mathbf{1 1 . 8 3}(10.16-13.77)$ & $\mathbf{5 . 1 3}(4.52-5.84)$ \\
\hline $\mathbf{6 - O M e}$ & $\mathbf{7 . 8 5}(7.66-8.04)$ & $\mathbf{1 . 6 5}(1.15-2.36)$ & $\mathbf{5 . 0 4}(3.48-7.31)$ & $\mathbf{1 . 8 7}(1.74-2.01)$ \\
\hline $\mathbf{6 - M e}$ & $\mathbf{2 . 0 2}(1.87-2.18)$ & $\mathbf{0 . 5 4}(0.53-0.54)$ & $\mathbf{2 . 0 0}(1.82-2.19)$ & $\mathbf{0 . 6 1}(0.57-0.65)$ \\
\hline $\mathbf{6 - M e}$ & $\mathbf{9 . 7 9}(9.23-10.39)$ & $\mathbf{3 . 1 8}(2.64-3.83)$ & $\mathbf{1 5 . 4 7}(13.15-18.20)$ & $\mathbf{5 . 4 6}(5.12-5.82)$ \\
\hline $\mathbf{6 - M e}$ & $\mathbf{8 . 6 1}(8.46-8.77$ & $\mathbf{2 . 1 7}(1.78-2.66)$ & $\mathbf{7 . 3 5}(6.86-7.88)$ & $\mathbf{3 . 2 0}(3.03-3.39)$ \\
\hline $\mathbf{6 - M e}$ & $\mathbf{1 4 . 1 0}(13.08-15.19)$ & $\mathbf{3 . 7 5}(3.12-4.50)$ & $\mathbf{1 3 . 4 5}(11.58-15.61)$ & $\mathbf{5 . 1 3}(4.93-5.35)$ \\
\hline $\mathbf{6 - M e}$ & $\mathbf{7 . 4 7}(7.28-7.65)$ & $\mathbf{3 . 6 9}(2.90-4.70)$ & $\mathbf{5 . 1 3}(4.92-5.35)$ & $\mathbf{4 . 8 5}(4.56-5.16)$ \\
\hline $\mathbf{6 - M e}$ & $\mathbf{1 1 . 0 9}(10.47-11.75)$ & $\mathbf{2 . 4 1}(1.54-3.78)$ & $\mathbf{1 4 . 5 7}(12.54-16.92)$ & $\mathbf{4 . 3 7}(4.22-4.52)$ \\
\hline $\mathbf{6 - M e}$ & $\mathbf{9 . 1 3}(8.88-9.39)$ & $\mathbf{2 . 0 3}(1.50-2.75)$ & $\mathbf{1 5 . 3 0}(14.53-16.12)$ & $\mathbf{4 . 8 6}(4.74-4.99)$ \\
\hline Dox & $\mathbf{0 . 7 9}(0.41-1.55)$ & $\mathbf{1 . 1 6}(0.91-1.47)$ & $\mathbf{2 . 1 9}(1.76-2.73)$ & $\mathbf{2 . 6 0}(2.05-3.30)$ \\
\hline
\end{tabular}

In order to evaluate the potential of the newly prepared protoflavone derivatives to overcome efflux-mediated multi-drug resistance (MDR), further collaborative studies were performed. The cytotoxicity of compounds (1, WYC0209, 31-44, 81-100 and 66-74) were tested in collaboration with Prof. Dr. Joseph Molnár and Dr. Ana Martins (Department of Medical Microbiology and Immunobiology, University of Szeged, Szeged, Hungary), utilizing two non- 
MDR/MDR cancer cell line pairs (parental L5178 mouse lymphoma cells and L5178 1 cells engineered to overexpress the human $\mathrm{ABCB} 1$ protein, and parental MCF-7 breast cancer cells and the doxorubicin resistant derivative MCF-7 ${ }_{\text {Dox }}$, overexpressing P-gp) [II,VII]. The fraction of $\mathrm{IC}_{50}$ values obtained in P-gp negative vs. positive cells served as a quantification of the MDR selective effect (selectivity ratio, $\mathrm{SR}$ ). Accordingly, $\mathrm{SR} \leq 0.5$ indicated that the compound is subject to $\mathrm{P}$-gp-mediated resistance, whereas $\mathrm{SR} \geq 2$ suggested that the P-gp expressing cells demonstrate collateral sensitivity against the tested protoflavone derivative. A graphical interpretation of the MDR selective cytotoxicity of each compound on the above-mentioned two susceptible/MDR cell line pairs is presented in Figure 7; detailed data is available in tabular form as Annex, Table A1.
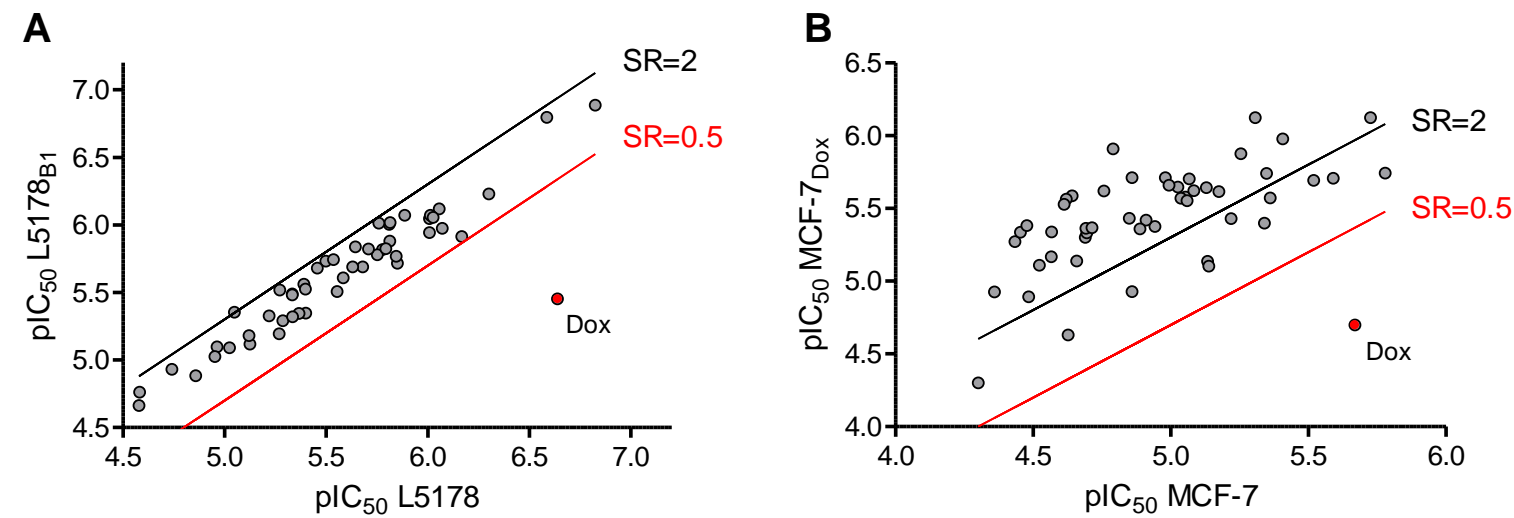

Figure 7. Cytotoxicity of protoflavones (1, WYC0209, 31-44, 81-100 and 66-74) . $\mathrm{pIC}_{50}$ values were derived from dose response curves obtained from cell viability experiments on L5178/L5178B1 mouse lymphoma cells (A) and MCF-7/MCF-7 Dox $_{\text {B }}$ cells (B). SR=Selectivity Ratio, calculated as $\mathrm{IC}_{50}{ }^{\text {nonMDR }} / \mathrm{IC}_{50}{ }^{\mathrm{MDR}}$; $\mathrm{n}=3-4$; Dox: doxorubicin.

The above results indicate that the synthesized protoflavone derivatives possess significant toxicity in the two cell line pairs. Interestingly, MCF7 cells were in general more resistant. Whereas the expression of ABCB1 did not modify the sensitivity of L5178 $\mathrm{B}$ cells in comparison with the parental L5178 cell line, MCF-7 $7_{\text {Dox }}$ cells showed collateral sensitivity to several derivatives, with SR values exceeding 5 in the case of compounds $(36,65-68,70)$.

To substantiate the role of $\mathrm{ABC}$ transporters in the MDR-selective toxicity of the compounds, additional MDR models were included in the study in collaboration with Dr. Gergely Szakács (Institute of Enzymology, Research Centre for Natural Sciences, Hungarian Academy of Sciences, Budapest Hungary). The cytotoxic activity of compounds (38-44, WYC0209, 31-37, 68-73 and 73) were tested in four additional MDR/sensitive cell line pairs, including A431, 
$\mathrm{A} 431_{\mathrm{B} 1}, \mathrm{~A} 431_{\mathrm{G} 2}, \mathrm{MES}-\mathrm{SA}, \mathrm{MES}-\mathrm{SA} / \mathrm{Dx} 5, \mathrm{~KB}-3-1$ and KB-V1. The results are shown in Figures 8-9; detailed data are available as Annex, Tables A2 and A3).

The tested protoflavone derivatives were equally toxic to $\mathrm{A} 431, \mathrm{~A} 431_{\mathrm{G} 2}$ or $\mathrm{A} 431_{\mathrm{B} 1}$ cells $\left(\mathrm{IC}_{50}\right.$ values ranged from $0.60 \mu \mathrm{M}$ to $7.27 \mu \mathrm{M}$ ), with the exception of protoapigenone (1), suggesting that the compounds tested herein are able to bypass $\mathrm{ABCB} 1$ or $\mathrm{ABCG} 2$. Resistance of $\mathrm{A} 431_{\mathrm{G} 2}$ cells to compound $\mathbf{2}$ was abolished in the presence of tariquidar, confirming that protoapigenone is an $\mathrm{ABCG} 2$ substrate (Figure 8).
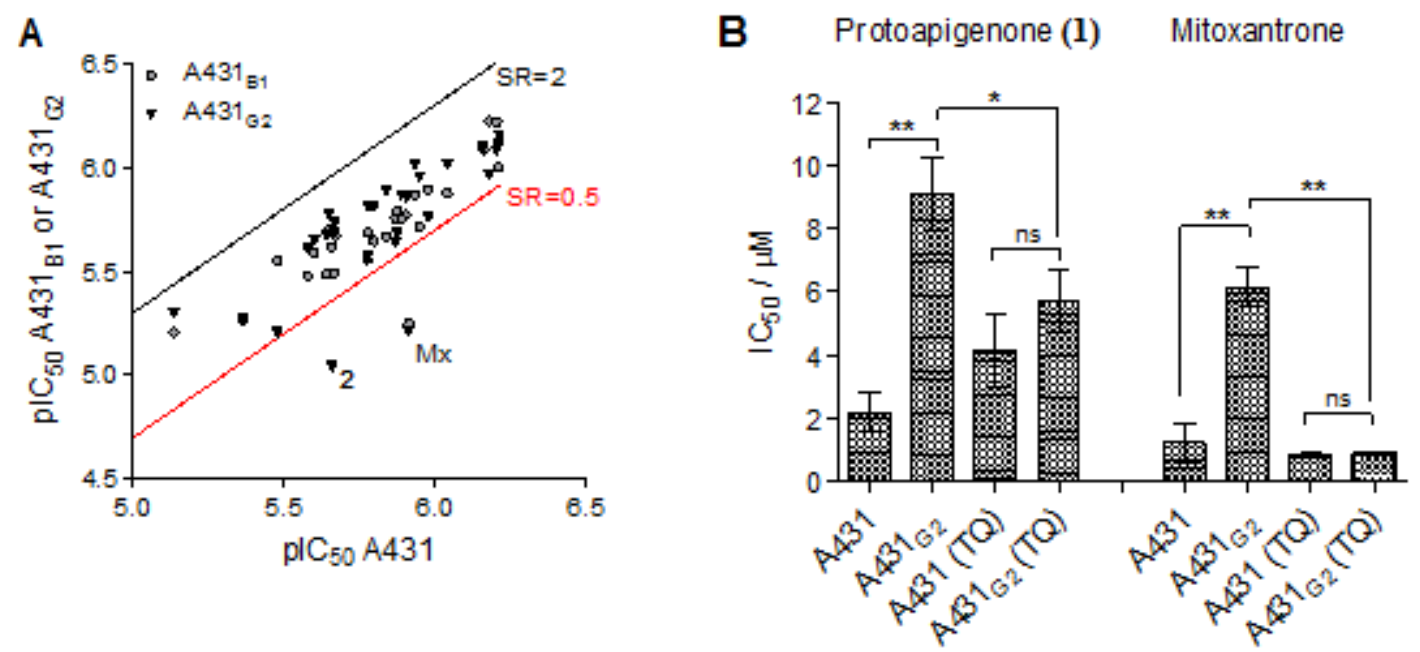

Figure 8. A. Cytotoxic activity of selected protoflavones against A431 (parental) cell line and two MDR derivatives engineered to overexpress ABCB1 (A431 1 1) or ABCG2 (A431 $1_{\mathrm{G} 2}$ ). $\mathrm{SR}=$ Selectivity Ratio, calculated as $\mathrm{IC}_{50}{ }^{\text {sensitive }} / \mathrm{IC}_{50}{ }^{\mathrm{MDR}} ; \mathrm{n}=3-4 ; \mathrm{Mx}$ : mitoxantrone. B. $\mathrm{IC}_{50}$ values of 1 (protoapigenone) and mitoxantrone in $\mathrm{A} 431$ and $\mathrm{A} 431_{\mathrm{G} 2}$ cell lines in the presence and absence of $1 \mu \mathrm{M}$ tariquidar, an ABCG2 efflux inhibitor.
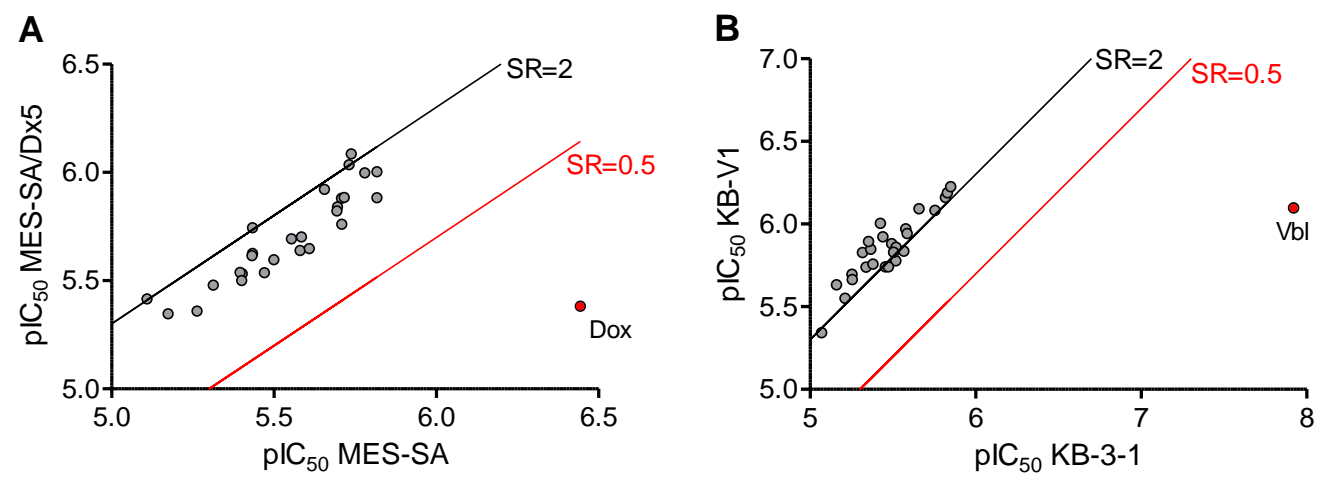

Figure 9. $\mathrm{pIC}_{50}$ values measured in doxorubicin-selected MES-SA/Dx5 (77) nd vinblastineselected KB-V1 (78) cells compared to the $\mathrm{pIC}_{50}$ values of MES-SA and KB-3-1 cells (A and $\mathbf{B}$, respectively). Two compounds were selectively toxic against both MES-SA/Dx5 and KBV1 cells ( $\mathbf{2}$ and $\mathbf{7 3}$ ), and none of the protoflavones showed substrate-like characteristics; Dox: doxorubicin, Vbl: vinblastine. 
Finally, we tested the interaction of the compounds with P-gp to reveal if any of the compounds inhibit drug efflux. ABCB1 function was characterized using the calcein accumulation assay [62]. Each derivative was assayed at two concentrations in the presence of the fluorescent indicator. Except for the 6-phenylprotoflavone series (66-71), which showed moderate inhibition at $20 \mu \mathrm{M}$ (14-46\%; for details see Annex, Table A4), none of the compounds inhibited the efflux of calcein AM by P-gp

In addition to the above results, an international research collaboration (Dr. Milica Pesic; Institute for Biological Research „Sinisa Stankovic”, Belgrade, Serbia) was utilized to test selected compounds (PA, PABu, PAPIII, BNFOH, 6MeOH, 6BrOH) on further four nonMDR/MDR cell line pairs, including NCI-H460 (human non-small cell lung carcinoma), DLD1 (human colorectal carcinoma), U87 (human glioma), C6 (rat glioma) and MRC-5 (human embryonal bronchial epithelial) cell lines, and their MDR counterparts NCI-H460/R (adapted to doxorubicin), DLD1-TxR and U87-TxR (adapted to paclitaxel), and RC6 (adapted to carmustine), respectively, and toxicity was also tested on HaCaT (normal human keratinocytes) and MRC-5 (embryonal bronchial epithelial) cell lines [VI] [63]. In this study, selective toxocity towards the MDR cell lines was found to correlate with the altered antioxidant defense of these cells: collateral sensitivity vs. cross-resistance to protoflavonoids appeared to be in line with that towards $\mathrm{H}_{2} \mathrm{O}_{2}$ induced oxidative stress, and several (necessarily adaptation-related, evolutionary) changes were evidenced in the redox homeostasis of the MDR cells as compared to that of their parental cell lines [VI] [63]. These changes all pointed in the same direction,

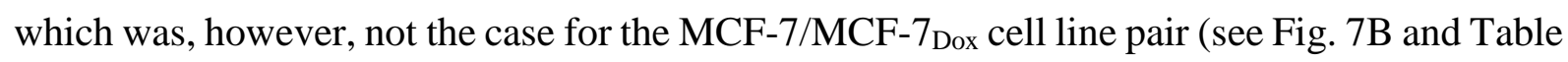
A1), whose collateral sensitivity was accompanied by resistance to $\mathrm{H}_{2} \mathrm{O}_{2}$ mediated oxidative stress. 


\subsection{Activity of selected compounds on xanthine oxidase}

Compounds (31-44 and 81-87) were also tested for their ability to inhibit xanthine oxidase (XO) [IV]. First, inhibition \% was determined at $0.1 \mathrm{mM}$, followed by taking dose-effect curve for compound (44) exerting a significant, nearly complete inhibition. The binding mode of (44) to the enzyme was also determined. Fig. 10 summarizes the results of these studies.
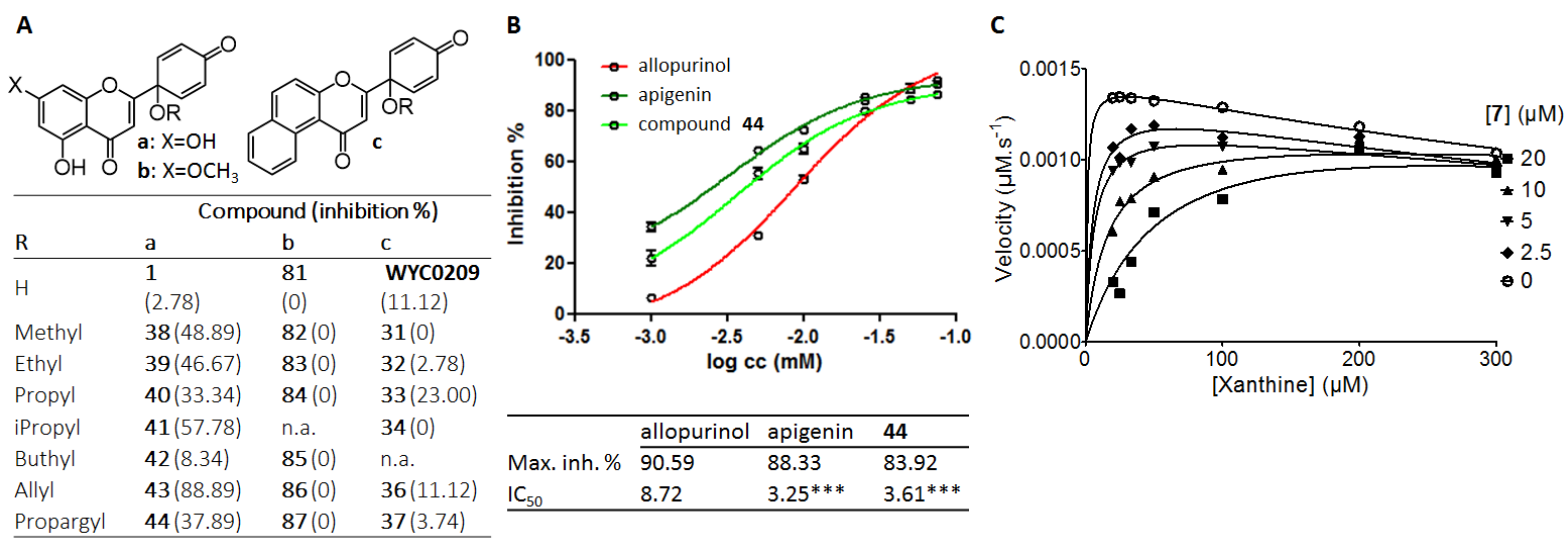

Figure 10. Activity of selected compounds 1, 31-44, 81-87 and WYC0209 on xanthine oxidase. A: inhibition \% of the compounds at $0.1 \mathrm{mM}$; B: dose-effect curves of PA-PIII in comparison with Ap and the positive control allopurinol; $\mathbf{C}$ : determination of the binding mode of (44) to the enzyme.

As seen from Fig. 10A, the genkwanin derivatives (group b) were inactive, whilst weak inhibition was found for some of the naphthoflavone derivatives (group c), and weak to moderate activity was observed for most of the protoapigenone analogs (group a). However, compound (44), protoapigenone 1'-O-propargyl ether, was found to inhibit the enzyme almost completely at the tested concentration. The dose-effect curve of this compound revealed a significantly stronger $\mathrm{IC}_{50}$ value that that of allopurinol (Fig 10B). The kinetic curve (Fig 10C) was found to be characteristic of substrate inhibition, that is, increasing the amounts of the substrate (here: xanthine) could also exert mild inhibition. Such a situation has already been described for XO (64). Furthermore, a global model with identical $\mathrm{V}_{\max }$ values for each curve was found $\left(1.376 \mathrm{nM} \mathrm{s}^{-1}\right)$ at $\mathrm{p}<0.05$, while no solutions were found when curve fitting criterion of a common $\mathrm{Km}$ value was set for all curves. Based on these thorough analyses, compound (44) is strongly suggested to be a competitive inhibitor of the enzyme.

The binding mode of (44) into the enzyme was investigated by in silico docking, using the $3 \mathrm{NVY}$ structure, corresponding to the crystal structure of $\mathrm{XO}$ in complex with quercetin 
(www.pdb.org). In this structure, quercetin is bound near the active center of the enzyme within the channel dedicated to the substrate. Since (44) is described here as a competitive inhibitor similar to quercetin, the $\mathrm{XO} /$ quercetin complex appears as a relevant model for further docking, that is, replacing quercetin by (44) and using the same active site for the docking procedure. iGEMDOCK, a docking and virtual screening tool utilizing the GEMDOCK scoring function was used,19,20 which combines an automatic preparation of the macromolecule around the bound ligands with docking and post-analysis. Re-docking the experimental ligand into the binding site resulted in a root mean square deviation (RMSD) value as low as $0.9149 \AA$ for the best-fit pose, which represented the most favorable van der Waals interactions. The results of this experiment and the best docking pose of (44), as well as its interactions with the enzyme are shown in Figure 11.
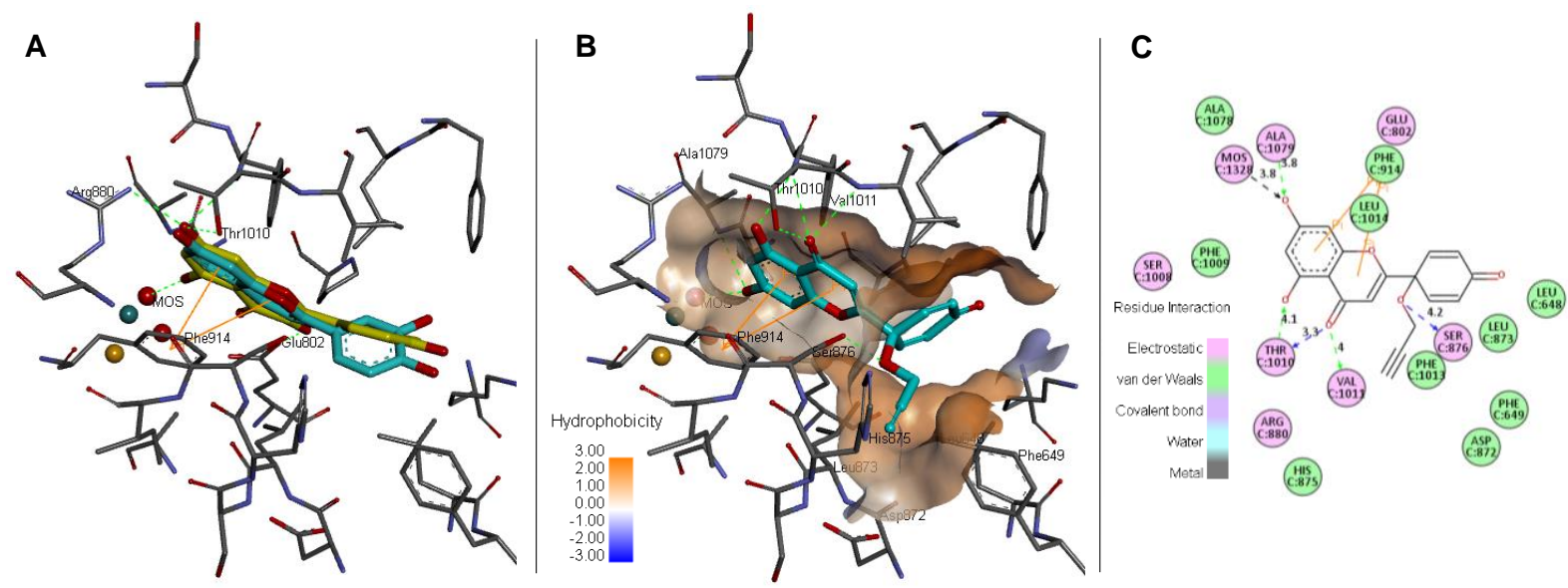

Figure 11. Results of the docking study. A: Overlapping of the re-docked quercetin residue (turquoise) with its experimental position (yellow, interactions shown to this position). B: Best docked position of (44) (turquoise) along with the hydrophobicity surface around the ligand at a probe radius of $1.1 \AA$. In $\mathbf{A}$ and $\mathbf{B}$, the viewing angles are identical, and $\mathrm{H}$-bonds and $\mathrm{p}-\mathrm{p}$ interactions are shown in green and orange, respectively. C: $2 \mathrm{D}$ Interpretation of the ligandresidue interactions between $3 \mathrm{NVY}$ and (44) including the distances of the H-bonds in $\AA$.

Of the ten possible solutions found in the docking study of (44), the best four represented very similar positions, with binding energies of $-126.88,-126.33,-118.68$ and $-117.50 \mathrm{kcal} / \mathrm{mol}$, respectively. In the case of the fifth best solution, the A and $\mathrm{C}$ rings of (44) almost perfectly overlapped with those of quercetin within the X-ray structure, resulting in a somewhat less favorable binding energy $(-95.37 \mathrm{kcal} / \mathrm{mol})$. The remaining five solutions showed much weaker binding to $3 \mathrm{NVY}(-68.11$ to $-16.73 \mathrm{kcal} / \mathrm{mol})$. 
In the most stable orientation of (44) in the XO active site, several H-bonds were observed (Fig. 11B), which differed from those existing in the presence of quercetin (Fig. 11A). This difference is mainly attributed to the flipped orientation of (44) with respect to that of quercetin, which modifies the $\mathrm{H}$-bonding possibilities with residues of the active site. Interestingly, due to these flip-flop orientations, the 5-OH and 7-OH groups have inverted roles in quercetin and compound (44). Concerning quercetin, the 5-OH group forms a $\mathrm{H}$-bond with the oxygen of the molybdopterin (MOS) residue, thus blocking its function. However, the 5-OH group of flavonoids is known to form a strong intramolecular H-bond with the keto group at C-4 [65] which consequently weakens the intermolecular H-bond with molybdopterine. In the case of compound (44), the 7-OH group forms this intermolecular $\mathrm{H}$-bond, which effectively enhances the binding to molybdopterin, and therefore the blocking of its function. This highlights the crucial role of the 7-OH group, fully rationalizing the dramatic loss of activity, e.g., from (44) to $(\mathbf{8 6})$ (Fig. 10).

The orientation of (44) in the XO active site is also stabilized by $\pi-\pi$ interactions between the $\mathrm{A}$ and $\mathrm{C}$ rings of the flavonoid and the aromatic ring of the Phe914 residue (Fig. 11B); these interactions are similarly observed for quercetin (Fig. 11A).

The most stable orientations found for (44) in the XO active site suggest a reasonable explanation for the importance of the propargyl side-chain: it perfectly fits into the hydrophobic pocket formed by the Leu648, Phe649, Asp872, Leu873 and His875 residues. Moreover, this orientation is also stabilized by a H-bond between the ether oxygen and Ser876. On the other hand, when the butyl ether derivative (42) was docked into 3NVY, its docking scores and positions could not explain its much lower activity compared to (44). Similar binding energies and orientations were found for these two compounds, and even the longer butyl ether sidechain was able to fit into the aforementioned pocket and form a H-bond with Ser876 at the same time. Side-chain length, on the other hand, might still be a limiting factor for these compounds to reach the active site through its relatively narrow access channel. This might explain the gradually decreasing XO inhibitor activity from the methyl to butyl derivatives (compounds 38-42) when tested at $0.1 \mathrm{mM}$. 


\section{Discussion}

\subsection{Synthesis}

By utilizing a simple oxidative de-aromatization by a common hypervalent iodine reagent similarly to the strategy published as a direct preliminary of this work [42], 15 new protoflavones were obtained from genkwanin, 4'-hydroxy-6-methylflavone. Following the observation on the apparent MDR selectivity of each derivative of the latter one (see below, Chapter 5.2.), the preparation of various 6-substituted derivatives was attempted.

In order to achieve this goal, the straightforward total-synthetic strategy reported by Lin et al. was utilized [38], following the preparation of those acetophenones that were commercially not available (46), (47). As an important difference to the reported total synthesis, efforts were made to optimize the ring closing step (i.e. chalchone to flavone). Even though related publications, including the one mentioned above, typically describe the use of a catalytic amount of iodine for the ring closure to obtain the flavone skeleton, utilizing a larger, 1 equivalent amount was found far more efficient. Following deprotection to obtain the 4'hydroxyflavone, the oxidative de-aromatization was achieved by utilizing 2 equivalents of PIFA in a 9:1 ratio mixture of acetonitrile and either water or the alcohol to be coupled at position 1' as an ether group. Structure elucidation of the protoflavones obtained this way was straightforward based on the mass and ${ }^{1} \mathrm{H}$ NMR spectra. The expected change in the molecular mass, and, in case of the 1 '- $O$-alkyl derivatives, the appearance of the characteristic ${ }^{1} \mathrm{H}$ NMR signals and coupling pattern of the side chain proved the successful linking of water or alcohol. The build-up of the protoflavone type B-ring was evidenced by the change in the coupling constant of the two dublets of $\mathrm{H}-3^{\prime} / \mathrm{H}-5^{\prime}$ and $\mathrm{H}-2^{\prime} / \mathrm{H}-6^{\prime}$ from ca. $8.8 \mathrm{~Hz}$ to ca. $10.0 \mathrm{~Hz}$, together with the remaining 3-H singlet and practically unchanged A-ring signals in the ${ }^{1} \mathrm{H}$ NMR spectrum.

\subsection{The apigenin - protoapigenone - apigenin redox cycle}

From our in silico and in vitro results it could be evidenced that, when scavenging ROS such as $\mathrm{OH}$ radicals, the well-known chemo-preventive agent apigenin [66] can yield the potent antitumor compound protoapigenone. Moreover, when studying the time-dependency of the formation of protoapigenone in the presence of excess $\mathrm{OH}$ radicals (i.e. Fenton reaction; see Fig. 6 on page 24) by using capillary electrophoresis, it was found that protoapigenone should indeed be a major primary product of such a scavenging event, just as it was previously predicted in silico. 
This is a particularly interesting finding that might completely change our understanding of how apigenin works in presence of oxidative stress (i.e. when it exerts its free radical scavenging antioxidant activity). Indeed, there are similarities between the bioactivities of these two compounds [67-68], which can likely be explained through a hypothesis considering protoapigenone as the "active form" of apigenin at least in terms of its antitumor and/or anticarcinogenic properties. Interestingly, a very similar redox circle was evidenced for estrone by Prokai et al. [69]. In this study, the antioxidant activity of estrone was explained through a pquinol A-ring containing derivative, which was found to rapidly transform back to estrone by enzymatic reduction, hence recovering the original compound able to scavenge further $\mathrm{OH}$ radicals [69]. Nevertheless, at the time of preparing this dissertation, we have not yet studied the enzymatic reduction of protoapigenone to apigenin, making it hard to make a judgment on the dynamics of this redox circle. The reaction with reduced glutathione was performed only in a test tube, and while this can prove that this reduction can take place in the presence of a relatively mild reducing agent, at this time no information is available on the involvement of enzymatic processes in this transition. It is also important to mention that only a limited data are available on the actual biological relevance of this redox cycle. Our preliminary experiments, however, suggest that oxidative stress can in fact modulate (increase) the in vitro antitumor activity of apigenin possibly through the formation of protoapigenone; further studies are necessary to clarify related biological aspects.

\subsection{Anticancer activity of protoflavones}

Our results on the cytotoxic activity of protoflavone derivatives against a diverse set of drug susceptible and MDR cancer cell lines provided a rich set of bioactivity data, and revealed several new structure-activity relationships (SAR). According to their B-ring substitution, cytotoxicity of the protoflavones on the utilized cell lines typically followed the SAR observed at the very beginning of our studies [I]: in most of the cases, 1'-OH substituted compounds were more toxic than those with $1^{\prime}$-alkoxy moieties and the isopropyl-ethers were the least cytotoxic derivatives. This, however, did not apply for protogenkwanone and its analogs (8187): protogenkwanone $1^{\prime}$ - $O$-methylether (82) exerted a stronger activity on the mouse lymphoma cells than (81). Moreover, an at least two carbons long side-chain was necessary for this series of compounds to be slightly toxic on MCF-7 cells. Presence of a non-branching propyl or butyl ether side chain at the $\mathrm{C}-1^{\prime}$ of protoapigenone (as in compounds $\mathbf{4 0}$ and $\mathbf{4 2}$ ) was found to be preferable for a strong cytotoxic activity [I]. This was also observed in the present study in most cell lines with the exceptions of the L5178 / L5178 1 and the MCF-7 / MCF-7 Dox 
cell line pairs. The introduction of a 1'-benzyloxy moiety (as in compound 45) to protoapigenone also resulted in an increased toxicity in the A431 cell line and its MDR subcell lines, as well as in the KB-3-1 and KB-V1 cells. This provides further evidence for the importance of the size and/or lipophilicity of the substituent at $\mathrm{C}-1^{\prime}$ and suggests that a larger branching and/or unsaturated alkyl side-chain might also lead to an increased cytotoxicity, despite the generally lower activity of the 1 '- $O$-isopropyl substituted derivatives as compared to those with linear alkyl chains.

Studies on the new compounds' activity against MDR cancer cell lines was initiated by investigating a set of compounds (PA, BNF, GEN és 6Me) on the L5178 / L5178 B1 $_{\text {cell line }}$ pair. A mild, yet statistically significant selectivity was observed towards the MDR cancer cell line for the 6-methyl substituted derivatives and none of the other compounds. This was a particularly interesting and important finding. Multi-drug resistance can emerge as a result of reduced uptake or increased efflux of cytostatic agents - the latter is mediated by ATP-bindingcassette $(\mathrm{ABC})$ proteins, primarily by P-glycoprotein (P-gp; ABCB1) and ABCG2, which confer resistance to a wide variety of compounds [7] [71]. There is a constant need for novel chemotherapeutics with marked and selective antitumor activity that can overcome resistance mediated by these transporters. The unfavorable prognostic impact of P-glycoprotein expression in several cancers has prompted overwhelming research efforts aimed at the clinical development of high affinity efflux inhibitors that were shown to overcome MDR in in vitro models. Unfortunately, even after decades of intensive research, a clinically effective inhibitor has not been identified. Recently, the discussion has shifted to alternative strategies, either to bypass the transporters or to exploit the collateral sensitivity (CS) of MDR cells [72]. Recent discoveries have shown that it is possible to invert the selective advantage of resistant cells to reverse the evolution of resistance [73].

According to the above, our synthetic efforts have been redirected towards the synthesis of new derivatives with various A-ring substituents, with a particular attention to position C-6, and investigating the compounds' in vitro anticancer activity on drug susceptible / MDR cell line pairs. In order to set a criterion for CS stricter than statistical significance, we decided to apply a two-fold threshold, namely CS was considered relevant if a compound exerted twice as strong activity on the MDR cell line than on its drug susceptible counterpart. Similarly, crossresistance $(\mathrm{CR})$ to a compound was considered relevant if its activity was two times weaker on the resistant cell line than on the parental one. 
Our attempt to increase the above-mentioned mild selective toxicity of 6-methylprotoflavones (88-94) on the ABCB1 transfected L5178 cells [II] by introducing various C-6 substituents revealed one single compound reaching our chosen threshold of relevance against the $\mathrm{L}_{5178_{\mathrm{B} 1}}$ cells, 6-methoxyprotoflavone 1 '-O-allylether (99). On the other hand, while all 6-methoxy compounds also showed tendency for such selectivity, other new derivatives showed decreased selective cytotoxicity against this cell line (Table A1). Similarly, none of the tested compounds, including 6-methylprotoflavone (88), showed (higher than two-fold) selective toxicity against $\mathrm{ABCB} 1$ transfected cell lines, including $\mathrm{A} 431_{\mathrm{B} 1}$ (Table A2) and MDCK- $\mathrm{II}_{\mathrm{B} 1}$ (not shown). Despite the equal toxicity of the studied compounds on parental and P-gp transfected cell lines, several derivatives proved selectively toxic against MDR cell lines overexpressing P-gp as a result of long-term drug selection. In particular, MCF-7 $7_{\text {Dox }}$ cells (adapted to doxorubicin) showed collateral sensitivity to most compounds except for $(34,39$, WY C0209, 69 and 75-80) with structural differences of the A-ring clearly influencing activity (Table A1). Compound (72), 6-phenylprotoflavone, for example, showed a remarkable, 13.2 fold selective cytotoxicity, while its $\beta$-naphthoflavone analog (WYC0209), where a fused aromatic ring is connected to the A-ring at the C-5/C-6 position, was non-selective. By comparing the selectivity ratios of compounds with different C-6 substituents, a clear SAR of the following order was observed: $\mathrm{Ph}>\mathrm{Me}>\mathrm{OMe} \approx \mathrm{Pentyl}$, while the ethoxy substituted protoflavones (75-80) and the ethyl substituted compound (72) were non-selective. No such general SAR could be concluded for the $\mathrm{C}-1^{\prime}$ substituents, except for the lower selectivity observed for the isopropyl ether derivatives (41) and (70). Interestingly, in case of the MES-SA / MES-SA/Dx5 cell line pair where the MDR sub-cell line was also obtained by adaptation to doxorubicin (74) collateral sensitivity was observed only for the classical, 1'-OH containing protoflavones $(\mathbf{1}, \mathbf{6 6}, 81$ and $\mathbf{8 8}$, but SR was below threshold for $\mathbf{3 1}$ and $\mathbf{8 2}$ ) and not for any of the $1^{\prime}$ - $O$-alkylprotoflavones. Furthermore, the KB-V1 cell line, obtained from KB-3-1 by adaptation to vinblastine [75], also presented marginal CS towards most of the protoflavones, although SR values for several compounds fell just below the 2-fold threshold (Figure 3B, Table S3).

Statistical significance of the SAR was tested from two angles. Compounds were grouped either according to their A-rings or their substituents at C-1', and the SR values of these groups were compared by one-way ANOVA ${ }^{2}$ followed by Bonferroni's post hoc test. No differences were observed for the $\mathrm{C}-1^{\prime}$ substituents on any of the cell lines, not even when the data were

\footnotetext{
${ }^{2}$ Each group containing at least 7 data points passed the Shapiro-Wilk normality test, suggesting the normal distribution of SR values under the influence of the presented chemical variations. Therefore, ANOVA is suitable for the statistical evaluation of these datasets.
} 
normalized to the average of their corresponding series (i.e. analogs with the same A-ring). However, the different A-ring containing protoflavone derivatives showed significant differences in their SR values on the MCF-7 / MCF-7 $7_{\text {Dox }}$ cell line pair; results are presented in Fig. 5.

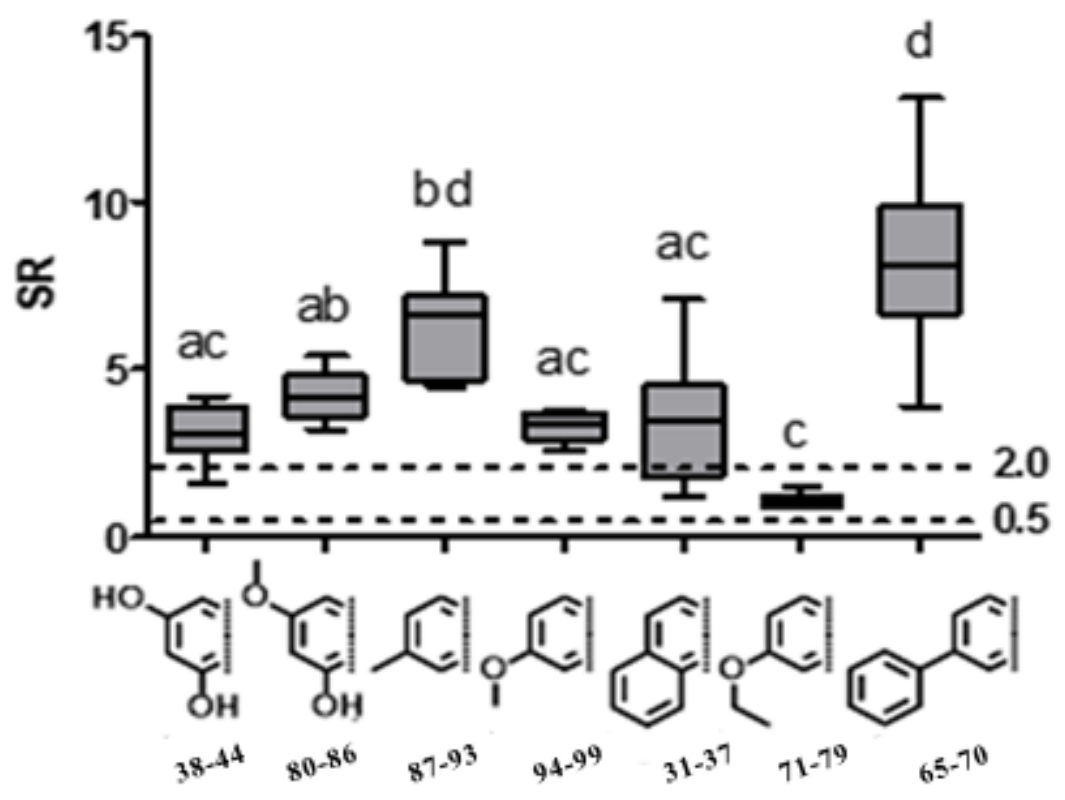

Figure 12. Selectivity ratio (SR) values for protoflavone analogs containing the same A-rings on the MCF-7 / MCF-7 ${ }_{\text {Dox }}$ cell line pair. Box-and-whisker plots represent medians, first and third quartiles and ranges; $\mathrm{SR} \geq 2.0$ and $\mathrm{SR} \leq 0.5$ represent $\mathrm{CS}$ and $\mathrm{CR}$, respectively; different lower case letters represent datasets with statistically significant differences (i.e. groups with overlap in their marking are not significantly different) at $\mathrm{p} \leq 0.05$ by one-way ANOVA followed by Bonferroni's post-hoc test.

Collateral sensitivity is causally linked to the adaption of MDR cells to a chemotherapeutic and may involve metabolic modifications, the up-regulation of receptors [76] or the modulation of the redox homeostasis [77]. One limitation of studies relying on MDR cell lines is that the contribution of MDR pumps, versus other acquired cellular alterations, cannot be delineated [78] [79] [80]. Collateral sensitivity of the MDR cell lines analyzed in this study indicates that resistance to doxorubicin or vinblastine may result in cellular alterations that render the cells susceptible to the protoflavone derivatives. However, in contrast to MDR-selective compounds [81], protoflavone derivatives do not selectively target cells engineered to overexpress Pglycoprotein, suggesting that the increased toxicity observed in the MDR cells is not conferred by the efflux pumps. This was also supported by our observation that selectivity ratios did not 
decrease significantly when compounds $\mathbf{1 , 8 1}$, and $\mathbf{8 8}$ were tested on the MES-SA / MES$\mathrm{SA} / \mathrm{Dx} 5$ cell line pair in the presence of tariquidar (data not presented).

It is important to point out that the lack of cross-resistance to most protoflavones in all MDR cell lines studied here indicates that these compounds can overcome MDR through bypassing efflux that is mediated by ABCB1 or ABCG2. SAR of the cytotoxic activity concerning the 6substituents appears to differ from cell line to cell line, for example an order of $\mathrm{Me}>\mathrm{Et} \approx$ Pent $\approx \mathrm{Ph}>\mathrm{Br}>\mathrm{OEt}>\mathrm{OMe}$ can be recognized on L5178 and L5178 ${ }_{\mathrm{B} 1}, \mathrm{Pent}>\mathrm{Ph} \approx \mathrm{Me}$ on KB-3-1 and KB-V1, while similar activities are exerted by 6-pentyl (73), 6-phenyl (66) and 6-methyl (88) compounds on MES-SA and MES-SA/Dx5. From a general overview, 6-alkyl substituted protoflavones appear to be somewhat more favorable anticancer agents over 6-alkoxy ones, even though nearly all compounds presented here can be considered as valuable leads against resistant cancers. As an interesting exception to this, however, resistance to protoapigenone (1) was observed in the $\mathrm{ABCG} 2$ transfected $\mathrm{A} 431_{\mathrm{G} 2}$ cell line, and a tendency for marginal resistance appeared also to its $1^{\prime}-O$-alkyl ethers. Resistance of this cell line to protoapigenone (1) markedly decreased in the presence of tariquidar, strongly suggesting that compound (1) is an ABCG2 substrate. ABCG2 did not confer resistance towards any of the other compounds including protogenkwanone (80), which differs from 1 only in its 7-methoxy group. This suggests that a non-substituted phenolic $\mathrm{OH}$ group at $\mathrm{C}-7$ is necessary for protoflavones to be recognized by this transporter.

\subsection{Xanthine oxidase inhibition by protoflavonoids}

Xanthine oxidase (XO), a molybdenum-containing metalloenzyme, is well known for its key role in the catabolism of purines, during which hypoxanthine and xanthine are transformed into uric acid. Elevated levels of uric acid lead to various pathological events including gout and the related formation of kidney stones. Moreover, although uric acid is the most abundant antioxidant in the human body, high XO activity makes a major contribution to oxidative stress by also producing reactive oxygen species (ROS) such as hydrogen-peroxide and superoxide anion radical [82] [83]. Despite their various problematic adverse effects, the purine derivative allopurinol and its active metabolite, oxipurinol, have long been nearly the exclusive choices for therapeutic XO inhibition. Thanks to the significant efforts on the search for new, nonpurine type XO inhibitors, several different classes of compounds have been revealed as possible alternatives: Febuxostat [2-(3-cyano-4-isobutoxyphenyl)-4-methyl-1,3-thiazole-5carboxylic acid], a non-competitive inhibitor of the enzyme, is probably the most important example to mention due to its recent approval by the FDA [84]. 
Another prospective class of non-purine type XO inhibitors is that of flavonoids, many of which have long been described as efficient inhibitors of the enzyme [85]. In accordance with our aim to search for non-cytotoxicity related bioactivities of protoflavones, XO inhibitory activity was tested for selected compounds.

A large body of scientific literature on flavonoids as XO inhibitors has previously been published. Prior to our study, all established structure-activity relationships (SAR) of these compounds were highly consistent with the following conclusions: $i$. structure of efficient inhibitors allows them to take a planar shape (i.e. no $\mathrm{sp}^{3}$ carbons are present; flavones are far more active than flavanones), ii. OH-groups at C-5 and C-7 are important for strong activity, and iii. flavonols, isoflavones and anthocyanodines are less active than flavones [86] [87] [88] [89] [90]. Accordingly, the surprisingly strong XO inhibitory activity observed for compound (44), the 1'-propargylether derivative of protoapigenone, allowed us to partially re-write the SAR accepted for flavonoids in this regard. Since the enzyme kinetics studies revealed that compound (PA-PIII) acts as a competitive inhibitor of $\mathrm{XO}$, in silico docking was expected to provide valuable insights as to how this compound can exert its activity at the active center of the enzyme.

The observed interactions (see chapter 4.5) provided a rational explanation how this compound can be anchored inside the channel through which the ligand should approach the molybdenum active center. Based on our results, the "planar structure" criterion no longer appears as a major prerequisite for efficient $\mathrm{XO}$ inhibitor activity of flavonoid derivatives; unlike the case of flavanones, if loss of the $\pi$-conjugation from the B- to the C-ring occurs at $\mathrm{C}-1$ ', the activity does not necessarily decrease as compared to flavones. Although the structure of protoapigenone 1'-O-propargyl ether certainly represents a rather special case among flavonoids, revision of this globally accepted structure-activity relationship might open novel directions for designing potent and safe XO inhibitors from this compound class. The planarity of the A and C moiety appears mandatory to favor intermolecular $\pi$ - $\pi$ stacking, but flexibility is permitted for the moiety attached to C-2. For example, enhancing the binding to the Leu648Phe649-Asp872-Leu873-His875 hydrophobic pocket is a relevant strategy to improve selectivity of flavonoid-type XO-inhibitors. 


\section{Summary}

By applying a straightforward synthetic strategy, combining semi-synthesis from naturally occurring 4'-hydroxyflavones and a 4-7 steps total synthetic approach, fifty-two protoflavone derivatives including 50 new compounds were prepared during this study. Chemical diversity of these compounds includes various A-ring substitution patterns (5,7-dihydroxy-, 5-hydroxy7-methoxy-, 6-methyl-, 6-methoxy-, 6-ethoxy-, 6-ethyl, 6-penthyl-, 6-bromo-, and 6-phenylsubstituted compounds as well as analogs of $\beta$-naphthoflavone) and various substituents at position C-1' (OH, $O$-Me, $O$-Et, $O$-Pr, $O$-iPr, $O$-Bu, $O$-allyl, $O$-propargyl and $O$-benzyl).

In silico DFT calculations revealed the possibility of an apigenin-protoapigenone transformation upon $\mathrm{OH}$ radical scavenging by the well-known antioxidant and chemopreventive agent apigenin. This was further supported by HPLC analyses of the reaction mixture obtained from the Fenton reaction of apigenin, where traces of protoapigenone were identified. By means of direct CE measurements on such mixtures, it can be concluded that protoapigenone is likely a major bioactive metabolite of apigenin whenever such a scavenging event takes place. Protoapigenone could also be reduced back to apigenin by an overnight incubation with reduced glutathione, an abundant intracellular antioxidant, which is evidence for the existence of an apigenin-protoapigenone-apigenin redox cycle. Further studies are necessary to clarify the biological relevance of this phenomenon.

The synthesized protoflavones were tested for their in vitro anticancer activity on a diverse set of drug susceptible and multi-drug resistant cell lines, and several new structure-activity relationships were revealed. The ability of protoflavones to evade efflux-mediated MDR was confirmed both in $\mathrm{ABCB} 1$ and $\mathrm{ABCG} 2$ expressing cell lines, with the exception of protoapigenone, which was identified as an ABCG2 substrate. MDR selective cytotoxicity was observed for most of the tested protoflavones in a breast cancer cell line adapted to doxorubicin (MCF-7 ${ }_{\text {Dox }}$ ) and SAR revealed importance of the A-ring substitution, while in the uterine sarcoma MES-SA/Dx5, another doxorubicin-selected cell line, only the 1'-OH containing compounds showed relevant selectivity. Since overexpression of neither ABCB1 nor ABCG2 sensitized the cells, it could be concluded that the MDR-selective cytotoxicity of protoflavones is connected to other changes accompanying acquired drug resistance. As an example to this, a smaller set of compounds were found to selectively kill drug-adapted MDR cancer cells whose antioxidant defense decreased during their evolution, and cross-resistance was observed with a cell line that had increased defense in this regard as compared to its parental cell line. 
Finally, protoapigenone 1 '- $O$-propargylether was identified as an efficient competitive inhibitor of xanthine oxidase (XO), a key enzyme of the purine metabolism involved in the pathomechanism of gout and many chronical diseases through its role in producing reactive oxygen species. This finding revealed for the first time that $\mathrm{sp}^{3}$ configuration is permitted for $\mathrm{C}-1$ ' for a flavonoid-type XO inhibitor, in contrast with the generally accepted SAR stating that the ability for adopting a planar structure is a must for such an activity. In silico docking studies revealed a flip-flop orientation of this compound as compared to that of quercetin, and provided a reasonable explanation for the role of the propargyl side chain, fitting perfectly into the hydrophobic pocket formed by the Leu648, Phe649, Asp872, Leu873 and His875 residues.

Altogether, this work on protoflavonoids provided several new derivatives with a natural product-inspired scaffold, provided new insights into their formation, and allowed us to obtain rich bioactivity and SAR data related to these compounds. Studies on their bioactivity from several angles outlined a number of promising research directions for the future, including, but not limited to, the possible use of protoflavone analogs as new lead compounds against multidrug resistant cancer. 


\section{Acknowledgments}

First of all, I would like to express my warmest thanks to my supervisor, Dr. Attila Hunyadi (Department of Pharmacognosy, University of Szeged, Hungary), and my co-supervisors Prof. Fang-Rong Chang and Prof. Yang-Chang Wu (Graduate Institute of Natural Products, Kaohsiung Medical University, Taiwan) for directing my Ph.D. work. I am thankful to Prof. József Molnár, Dr. Ana Martins, (Department of Medical Microbiology and Immunobiology, Faculty of Medicine, University of Szeged, Szeged, Hungary) and Dr. Gergely Szakács, and Szilárd Tóth (Institute of Enzymology, Research Centre for Natural Sciences, Hungarian Academy of Sciences, Budapest, Hungary) for performing bioactivity tests related to MDR cancer; to Prof. György Falkay (Department of Pharmacology, Faculty of Pharmacy, University of Szeged, Szeged, Hungary) for the Xantin oxidase assay; and to Dr. Krisztina Németh (Institute of Organic Chemistry, Research Centre for Natural Sciences, Hungarian Academy of Sciences, Budapest, Hungary) for the capillary electrophoresis experiments. I owe a special thanks to Prof. Patrick Trouillas, Dr. Gabin Fabre (Faculty of Pharmacy, University of Limoges, Limoges, France) for the in silico experiments. My thanks are likewise to all my colleagues in the Institute of Pharmacognosy (Szeged, Hungary) and in the Gradute Institute of Natural Products (Kaohsiung, Taiwan). I am especially grateful to Ibolya Hevérné Herke, Norbert Kúsz, Máté Vágvölgyi, Attila Horváth, Dr. Wan-Chun. Lai, and Dr. Da-Wei Chuang for their help and support. I owe special thanks to Prof. Leonard Amaral for the valuable advices. Finally I would like to thank to the Richter Gedeon Plc. and to the European COST Association for financial supporting my work, and I acknowledge the National Research, Development and Innovation Office, Hungary (NKFIH; K119770) for funding our research on protoflavone derivatives. 


\section{References}

1. Di Carlo G, Mascolo N, Izzo AA, Capasso F. Flavonoids : old and new aspects of a class of natural therapeutic drugs. Life Sci. 1999, Vol. 65, pp. 337-353.

2. L, Le Marchand. Cancer preventive effects of flavonoids-a review. Biomed Pharmacother. 2002, Vol. 56, pp. 296-301.

3. Tapas AR, Sakarkar DM, Kakde RB. Flavonoids as Nutraceuticals: A Review. Trop J Pharm Res. 2008, Vol. 7(3), pp. 1089-1099.

4. Bentsáth A, Rusznyák ST, Szent-Györgyi A. Vitamin nature of flavones. Nature. 1936, Vol. 138(7), p. 798.

5. Ososki AL, Kennelly EJ. Phytoestrogens: a review of the present state of research.

Phytother Res. 2003, Vol. 17, pp. 845-869.

6. Hayashi K, Hayashi T, Otsuka K, Takeda Y. Antiviral activity of 5,6,7-trimethoxyflavone and its potentiation of the antiherpes activity of acyclovir. J Antimicrob Chemotherther. 1997, Vol. 39, pp. 821-824.

7. Fukushima S, Noro T, Saiki Y, Ueno A, Akahori Y. Constituents of Leptorumohra miqueliana. I. Structures of leptorumolin and leptorumol. Yakigaku Zasshi. 88 (9), 1968, pp. 1135-42.

8. Noro T, Fukushima S, Saiki Y, Ueno A, Akahori Y. Constituents of Leptorumohra miqueliana. II. Yakugaku Zasshi. 89(6), 1969, pp. 851-6.

9. Fukushima S, Noro T, Akahori Y, Saiki Y, Ueno A. Constituents of Leptorumohra miqueliana. III. Yakugaku Zasshi. 89(9), 1969, pp. 1272-5.

10. Murakami T, Kido T, Hori K, Satake T, Saiki Y, Chen, C-M. Chemical and chemotaxonomic studies of filices. LXVII. The distribution of a flavone with a modified Bring, protofarrerol and its derivates. Yakugaku Zasshi. 1987, 107, pp. 416-419.

11. Gerold Jerz, Reiner Waibel, Hans Achenbach. Cyclohexanoid protoflavanones from the stem-bark and roots of Ongokea gore. Phytochemistry. 66, 2005, pp. 1698-1706.

12. Giovana C. Freitas a, João M. Batista Jr. b, Gilberto C. Franchi Jr. c, Alexandre E. Nowill c. Cytotoxic non-aromatic B-ring flavanones from Piper carniconnectivum. Phytochemistry. 2014, Vol. 97, pp. 81-87.

13. Hauteville M, Chopin J, Geiger H, Schueler L. Protogenkwanin 4'-glucoside, a new type of natural flavonoid with a nonaromatic B-ring. Tetrahedron Lett. 1980, Vol. 21(13), pp. 1227-1230.

14. Hauteville M, Chopin J, Geiger H, Schuler L. Protogenkwanin, a new flavonoid from Equisetum arvense L. Tetrahedron. 1981, Vol. 37(2), pp. 377-381.

15. Stomberg R, Lundquist K, Hauteville M, Geiger H. Crystal structure of protogenkwanin, C16H14O6. J Crystallogr Spectrosc Res. 1991, Vol. 21(2), pp. 183-188. 
16. A, Klaus-Peter. Phenolic constitutents of the fern Phegopteris connectilis. Phytochemistry. 1999, Vol. 52, pp. 929-934.

17. Wada H, Fujita H, Murikami T, Saiki Y, Chen CM. Chemical and Chemotaxonomical Studies of Ferns. LXXIII.1) New Flavonoids with Modified B-Ring from the Genus Pseudophegopteris (Thelypteridaceae). Chem Pharm Bull. 1987, Vol. 35(12), pp. 4757-4762.

18. Pouny I, Etiévant C, Marcourt L, Huc-Dumas I, Batut M, Girard F, Wright M, Massiot G. Protoflavonoids from Ferns Impair Centrosomal Integrity of Tumor Cells. Planta Med. 2011, Vol. 77, pp. 461-466.

19. Lin AS, Chang FR, Yen HF, Björkeborn HF, Norlén P, Wu YC. Novel flavonoids of Thelypteris torresiana. Chem Pharm Bull. 2007, Vol. 55(4), pp. 635-637.

20. Fang W, Ruan J, Cai Y,Wei A, Zhou D, Zhang W. Flavonoids from the aerial parts of Macrothelypteris torresiana. Nat Prod Res. 2011, Vol. 25(1), pp. 36-39.

21. Liu H, Jiang C, Xiong C, Ruan J. DEDC, a new flavonoid induces apoptosis via a ROSdependent mechanism in human neuroblastoma SH-SY5Y cells. Toxicol In Vitro. 2012, Vol. 26(1), pp. 16-23.

22. Keiko T, Yasushi, Yuki K, Kensuke Y, Shigeru H, Hiroguki I, Yumi N, Noriyuki M, Mamou I, Norio o, Shinjiro I. Anti-inflammatory effects of green soybean extract irradiated with visible light. Sci Rep. 4, 2014, p. 4732.

23. Nikolic N, Cvetkovic D, Todorovic Z. A characterization of content, composition and antioxidant capacity of phephenolic compounds in celery roots. Ital J Food Sci. 2011, Vol. 23, pp. 214-219.

24. Giovana C. Freitas, João M. Batista Jr., Gilberto C. Franchi Jr., Alexandre E. Nowill, Lydia F. Yamaguchi, Janaina D. Vilcachagua, Denize C. Favaro, Maysa Furlan, Elsie F. Guimarães, Christopher S. Jeffrey, Massuo J. Kato. Cytotoxic non-aromatic B-ring flavanones from Piper carniconnectivum C. DC. Phytochemistry. 2014, Vol. 97, pp. 81-87.

25. Hauteville M, Chopin J, Geiger H, Schueler L. Protogenkwanin 4`-glucoside, a new type of natural flavonoid with a nonaromatic B-ring. Tetrahedron. 21(13), 1980, pp. 1227-1230.

26. Hauteville M, Chopin J, Geiger H, Schuler L. Protogenkwanin, a new flavonoid from Equisetum arvense L. Tetrahedron. 37(2), 1981, pp. 377-381.

27. Veit M, Bauer K, Beckert C, Kast B, Geiger H, Czygan FC. Phenolic characters of british hybrid taxa in equisetum. Biochem Syst Ecol. 1995, Vol. 23(1), pp. 79-87.

28. Lin AS, Chang FR, Wu CC, Liaw CC, Wu YC. New cytotoxic flavonoids from Thelypteris torresiana. Planta Med. 2005, Vol. 71, pp. 867-870.

29. A novel flavonoid from the root of Macrothelypteris torresiana. Tang Y, Fang W, Ma

YT, Cai YL, Ruan JL. 2009, Ching. Chinese Chem Lett, Vol. 20 (7), pp. 815-816.

30. A new flavonoid from Macrothelypteris torresiana. Tang Y, Xiong C, Zhou D, Wei A, Fu W, Cai Y, Ruan J. 2010, Chem Nat Comp, Vol. 46(2), pp. 209-211. 
31. Wu G, Wei A,Cai Y, Xiong C, Ruan J. Chemical constituents of Macrothelypteris oligophlebia and their antitumor activity in vitro and in vivo. Zhongguo Yaoxue Zazhi. 46(5), 2011, pp. 330-333.

32. Wei A, Zhou D, Xiong C, Cai Y, Ruan J. A novel non-aromatic B-ring flavonoid: Isolation, structure elucidation and its induction of apoptosis in human colon HT-29 tumor cell. Food Chem Toxicol. 49(9), 2011, pp. 2445-2452.

33. Wei A, Zhou D, Xiong C, Cai Y, Ruan J. A novel non-aromatic B-ring flavonoid: Isolation, structure elucidation and its induction of apoptosis in human colon HT-29 tumor cell via the reactive oxygen species-mitochondrial dysfunction and MAPK activation. Food Chem Toxicol. 2011, Vol. 49(9), pp. 2445-2452.

34. Wei A, Zhou D,Ruan J, Cai Y, Xiong C, Wu G. Anti-tumor and anti-angiogenic effects of Macrothelypteris viridifrons and its constituents by HPLC-DAD/MS analysis. $J$ Ethnopharmacol. 2012, Vol. 139(2), pp. 373-380.

35. Freitas GC, Batista JM Jr, Franchi GC Jr, Nowill AE, Yamaguchi LF, Vilcachagua JD, Favaro DC, Furlan M, Guimarães EF, Jeffrey CS, Kato MJ. Cytotoxic non-aromatic B-ring flavanones from Piper carniconnectivum C. DC. Phytochemistry. 2014, Vol. 97.

36. Jerz G, Waibel R, Achenbach H. Cyclohexanoid protoflavanones from the stem-bark and roots of Ongokea gore. Phytochemistry. 66 (14), 2005, pp. 1698-706.

37. Capistrano R, Wouters A, Fouberta K, Baldéc AM, Apers S, Lardon F, Pieters L, Exarchoua V. Phytochemical characterisation of a cytotoxic stem bark extract of Steganotaenia araliacea and identification of a protoflavanone by LC-SPE-NMR. Phytochemistry Letters. Vol. 12, 2015, pp. 119-124.

38. Lin AS, Nakagawa-Goto K, Chang FR, Yu D, Morris-Natschke SL, Wu CC, Chen SL, Wu YC, Lee KH. First Total Synthesis of Protoapigenone and its Analogs as Potent Cytotoxic Agents. J Med Chem. 2007, Vol. 50(16), pp. 3921-3927.

39. Chang HL, Su JH, Yeh YT, Lee YC, Chen HM, Wu YC, Yuan SSF. Protoapigenone, a novel flavonoid, inhibits ovarian cancer cell growth in vitro and in vivo. Cancer Lett. 2008, Vol. 267, pp. 85-95.

40. Chang HL, Wu YC, Su JH, Yeh YT, Yuan SSF. Protoapigenone, a Novel Flavonoid, Induces Apoptosis in Human Prostate Cancer Cells through Activation of p38 MitogenActivated Protein Kinase and c-Jun NH2-Terminal Kinase. J Pharmacol Exp Ther. 2008, Vol. 325, pp. 841-849.

41. Chiu CC, Chang HW, Chuang DW, Chang FR, Cheng YS, Tsai MT, Chen WY, Lee SS, Wang CK, Chen JY, Wang HM, Chen CC, Liu YC, Wu YC. Fern plant-derived protoapigenone leads to DNA damage, apoptosis, and $\mathrm{G}(2) / \mathrm{m}$ arrest in lung cancer cell line H1299. DNA Cell Biol. 28, 2009, pp. 501-506.

42. Hunyadi A, Chuang DW, Danko B, Chiang MY, Lee CL, Wang HC, Wu CC, Chang FR, Wu YC. Direct Semi-Synthesis of the Anticancer Lead-Drug Protoapigenone from Apigenin, and Synthesis of Further New Cytotoxic Protoflavone Derivatives. PLoS ONE. 2011, Vol. 6(8), p. e23922. 
43. Wang HC, Lee AYL, Chou WC, Wu CC, Tseng CN, Liu KYT, Lin WL, Chang FR, Chuang DW, Hunyadi A, Wu YC. Inhibition of ATR-Dependent Signaling by

Protoapigenone and Its Derivative Sensitizes Cancer Cells to Interstrand Cross-link-

Generating Agents In Vitro and In Vivo. Mol Cancer Ther. 2012, Vol. 11, pp. 1443-1453.

44. Chen HM, Chang FR, Hsieh YC, Cheng YJ, Hsieh KC, Tsai LM, Lin AS, Wu YC, Yuan

SS. A novel synthetic protoapigenone analogue, WYC02-9, induces DNA damage and apoptosis in DU145 prostate cancer cells through generation of reactive oxygen species. Free Rad Biol Med. 2011, Vol. 50, pp. 1151-1162.

45. Lapenna S, Giordano A. Cell cycle kinases as therapeutic targets for cancer. Nat Rev Drug Discov. 2009, Vol. 8, pp. 547-566.

46. Bolderson E, Richard DJ, Zhou BB, Khanna KK. Recent advances in cancer therapy targeting proteins involved in DNA double-strand break repair. Clin Cancer Res. 2009, Vol. 15, pp. 6314-6320.

47. Smith J, Tho LM, Xu N, Gillespie DA. The ATM-Chk2 and ATR-Chk1 pathways in DNA damage signaling and cancer. 2010, Vol. 108, pp. 73-112.

48. Ziwei Liu, Qianying Yuan, Xuenong Zhang, Chaomei Xiong, Pingping Xue, Jinlan Ruan. RY10-4, a novel anti-tumor compound, exhibited its anti-angiogenesis activity by downregulation of the HIF-1 and inhibition phosphorylation of AKT and mTOR. Cancer Chemother Pharmacol. 2012, Vol. 69, pp. 1633-1640.

49. Qianying Yuan, Shaoxin Cai, Xuenong Zhang, Ziwei Liu, Zhaoming Li, Xuelai Luo b. A new protoapigenone analog RY10-4 induces apoptosis and suppresses invasion through the PI3K/Akt pathway in human breast cancer. Cancer Letters. 2012, Vol. 324, pp. 210-220.

50. Pingping Xue, Yang Zhao, Yang Liu, Qianying Yuan, Chaomei Xiong, Jinlan Ruan. A novel compound RY10-4 induces apoptosis and inhibits invasion via inhibiting STAT3 through ERK-, p38-dependent pathways in human lung adenocarcinoma A549 cells.

Chemico-Biological Interactions. Vol. 209, pp. 25-34.

51. Ziwei Liu, Zhimei Liu, Xiulan Zhang, Pingping Xue, Heng Zhang. RY10-4 suppressed metastasis of MDA-MB-231 by stabilizing ECM and. Biomedicine \& Pharmacotherapy. 2014, Vol. 68, pp. 439-445.

52. Pingping Xue, Xiaofan Yang, Yang Liu, Chaomei Xiong, Jinlan Ruan. A novel compound RY10-4 downregulates P-glycoprotein expression and reverses multidrug-resistant phenotype in human breast cancer. Biomedicine \& Pharmacotherapy. 2014, Vol. 68, pp. 1049-1056.

53. Chen WY, Hsieh YA, Tsai CI, Kang YF, Chang FR, Wu YC, Wu CC. Protoapigenone, a natural derivative of apigenin, induces mitogen-activated protein kinase-dependent apoptosis in human breast cancer cells associated with induction of oxidative stress and inhibition of glutathione S-transferase $\pi$. Invest New Drugs. 29, 2011, pp. 1347-1359.

54. Tung CP, Chang FR, Wu YC, Chuang DW, Hunyadi A, Liu ST. Inhibition of the EpsteinBarr virus lytic cycle by. J Gen Virol. 2011, Vol. 92, pp. 1760-1768. 
55. Wu, Yang-Chang, et al. Compound for inhibiting glutathione s-transferase omega 1 activity, pharmaceutical composition containing thereof, and method for synthesizing the same . US20140221470 A1 U.S. Pat. Appl. Publ., Augustus 7, 2014.

56. Wu, Yang-Chang, et al. Compound for inhibiting activity of glutathione s-transferase omega 1 and preparation method thereof, and pharmaceutical compositions containing compound . WO2015085459 Al WO, június 18, 2015.

57. I. Pastan, I., et al. A retrovirus carrying an MDR1 cDNA confers multidrug resistance and polarized expression of P-glycoprotein in MDCK cells. Proc. Natl. Acad. Sci. U. S. A. 1988, Vol. 85, pp. 4486-4490.

58. Kars MD, Iseri OD, Gündüz U, Ural AU, Arpaci F, Molnár J. Development of rational in vitro models for drug resistance in breast cancer and modulation of MDR by selected compounds. Anticancer Res. 26, 2006, pp. 4559-4568.

59. L. Homolya L, Hollo M, Muller M, Mechetner EB, Sarkadi B. A new method for a quantitative assessment of P-glycoprotein-related multidrug resistance in tumour cells. A new method for a quantitative assessment of $P$-glycoprotein-related multidrug resistance in tumour cells. 73, pp. 849-855.

60. Danko B, Martins A, Chuang DW, Wang HC, Amaral L, Molnar J, Chang FR, Wu YC, Hunyadi A. In vitro cytotoxic activity of novel protoflavone analogs - selectivity against a multi-drug resistant cancer cell line. Anticancer Res. . 32, 2012, pp. 2863-2870.

61. Tu, Q.D., et al. Design and syntheses of novel N'-((4-oxo-4H-chromen-3-

yl)methylene)benzohydrazide as inhibitors of cyanobacterial fructose-1,6-/sedoheptulose-1,7bisphosphatase. Bioorg. Med. Chem. 2013, Vol. 21, pp. 2826-2831.

62. A new method for a quantitative assessment of P-glycoprotein-related multidrug resistance. L. Homolya, M. Hollo, M. Muller, E. B. Mechetner, B. Sarkadi. 73 : s.n., 1996, Br. J. Cancer. pp. 849-855.

63. Stankovic T, Danko B, Martins A, Dragoj M, Stojkovic, Isakovic A, Wang H-C, Wu Y-C, Hunyadi A, Pesic M. Lower antioxidative capacity of multidrug-resistant cancer cells confers collateral sensitivity to protoflavone derivatives. Cancer Chemotherapy and Pharmacology. 2015, Vol. 76, pp. 555-565.

64. Rubbo H, Radi R, Prodanov E. Substrate inhibition of xanthine oxidase and its influence on superoxide radical production. s.l. : Elsevier, 1991.

65. P Trouillas, P Marsal, D Siri, R Lazzaroni, JL Duroux. Chalcones: structural requirements for antioxidant, estrogenic and antiproliferative activities. Food Chemistry. 2006., Vol. 97 (4), pp. 679-688.

66. Patel D, Shukla S, Gupta S. Apigenin and cancer chemoprevention: progress, potential and promise (review). Int J Oncol. 2007, Vol. 30, pp. 233-45.

67. Wen-Ying Chen, Yu-An Hsieh, Ching-I Tsai, Ya-Fei Kang, Fang-Rong Chang, YangChang Wu, Chin-Chung Wu. Protoapigenone, a natural derivative of apigenin, induces mitogen-activated protein kinase-dependent apoptosis in human breast cancer cells associated 
with induction of oxidative stress and inhibition of glutathione S-transferase $\pi$. Investigational New Drugs. 2011, Vol. 29, pp. 1347-1359.

68. Hunyadi A, Martins A, Danko B, Chang FR, Wu YC. Protoflavones: a class of unusual flavonoids as promising novel anticancer agents. Phytochemistry Reviews. 2014, Vol. 13, pp. 69-77.

69. Prokai L, Prokai-Tatrai K, Perjesi P, Zharikova AD, Perez EJ, Liu R, Simpkins JW. Quinol-based cyclic antioxidant mechanism in estrogen neuroprotection. Proc Natl Acad Sci U S A. 2003, Vol. 100, pp. 11741-6.

70. M. M. Gottesman, T. Fojo, S. E. Bates. Multidrug resistance in cancer: role of ATPdependent transporters. Nat. Rev. Cancer 2002, Vol. 2, pp. 45-48.

71. G. Szakács, J. K. Paterson, J. A. Ludwig, C. Booth-Genthe, M. M. Gottesman. Targeting multidrug resistance in cancer. Nat. Rev. Drug Discov. 2006, Vol. 5, pp. 219-234.

72. G. Szakács, M. D. Hall, M. M. Gottesman, A. Boumendjel, R. Kachadourian, B. J. Day, H. Baubichon-Cortay, A. Di Pietro. Targeting the Achilles Heel of Multidrug-Resistant Cancer by Exploiting the Fitness Cost of Resistance. Chem. Rev. 2014, Vol. 114, pp. 57535774.

73. M. Baym, L. K. Stone, R. Kishony. Multidrug evolutionary strategies to reverse antibiotic resistance. Science . 2016, Vol. 351.

74. W. G. Harker, B. I. Sikic. Multidrug (Pleiotropic) Resistance in Doxorubicin-selected Variants of the. Cancer Res. 1985, Vol. 45, pp. 4091-4096.

75. D. W. Shen, C. Cardarelli, J. Hwang, M. Cornwell, N. Richert, S. Ishii, I. Pastan. Multiple drug-resistant human KB carcinoma cells independently selected for high-level resistance to colchicine, adriamycin, or vinblastine show changes in expression of specific proteins. J. Biol. Chem. 1986, Vol. 261, pp. 7762-7770.

76. L. Rickardson, M. Fryknäs, C. Haglund, H. Lövborg, P. Nygren, M. G. Gustafsson, A. Isaksson, R. Larsson. Cancer Chemother. Pharmacol. 2006, Vol. 58, pp. 749-758.

77. A. S. Goldsborough, M. D. Handley, A. E. Dulcey, K. M. Pluchino, P. Kannan, K. R. Brimacombe, M. D. Hall, G. Griffiths, M. M. Gottesman. Collateral Sensitivity of MultidrugResistant Cells to the Orphan Drug Tiopronin. J. Med. Chem. 54, 2011, Vol. 54, pp. $4987-$ 4997.

78. J. A. Ludwig, G. Szakács, S. E. Martin, B. F. Chu, C. Cardarelli, Z. E. Sauna, N. J. Caplen, H. M. Fales, S. V. Ambudkar, J. N. Weinstein, M. M. Gottesman. Selective Toxicity of NSC73306 in MDR1-Positive Cells as a New Strategy to Circumvent Multidrug Resistance in Cancer. Cancer Research. Vol. 66, 2006, pp. 4808-4815.

79. G. Szakács, J. P. Annereau, S. Lababidi, U. Shankavaram, A. Arciello, K. J. Bussey, W. Reinhold, Y. Guo, G. D. Kruh, M. Reimers, J. N. Weinstein, M. M. Gottesman. Predicting drug sensitivity and resistance: profiling $\mathrm{ABC}$ transporter genes in cancer cells. Cancer Cell. 2004, Vol. 6, pp. 129-137. 
80. D. Türk, M. D. Hall, B. F. Chu, J. A. Ludwig, H. M. Fales, M. M. Gottesman. Identification of Compounds Selectively Killing Multidrug-Resistant Cancer Cells. Cancer Res. 2009, Vol. 69, pp. 8293-8301.

81. A. Füredi, S. Tóth, K. Szebényi, V. F. S. Pape, D. Türk, N. Kucsma, L. Cervenák, J. Tóvári, G. Szakács. Identification and Validation of Compounds Selectively Killing Resistant Cancer. Mol. Cancer Ther. 2017, Vol. 16, pp. 35-44.

82. Terada, L. S., Leff, J. A., Repine, J. E. Measurement of xanthine oxidase in biological tissues. Methods Enzymol. 1990, Vol. 186, pp. 651-6.

83. Packer, L., Glazer. A. N. Academic Press: New York. 1990, Vol. 186, pp. 651-656.

84. FDA approves drug for gout. www.fda.gov/

NewsEvents/Newsroom/PressAnnouncements/ucm1. [Online] 2009.

85. Eiler, J. M. and Martin, G. J. J. Biol. Chem. 192, 1951, pp. 831-834.

86. Hayashi, T., et al. N. J. Nat. Prod. 1988, Vol. 51, pp. 345-348.

87. Chang, W. S., et al. H. C. Anticancer Res. 1993, Vol. 13, pp. 2165-2170.

88. Cos, P., et al. Structure-Activity Relationship and Classification of Flavonoids as Inhibitors of Xanthine Oxidase and Superoxide Scavengers. J. Nat. Prod. 1998, Vol. 61, pp. 71-76.

89. Nagao, A., Seki, M. and Kobayashi, H. Biosci. Inhibition of Xanthine Oxidase by Flavonoids. Biotechnol. Biochem. 1999, Vol. 63, pp. 1787-1790.

90. Van Hoorn, D. E. C., et al. Accurate prediction of xanthine oxidase inhibition based on the structure of flavonoids. K. Eur. J. Pharmacol. 2002, Vol. 451, pp. 111-118.

91. Noro T, Fukushima S, Saiki Y, Ueno A, Akahori Y. Studies on the constituents of Leptorumohra miqueliana H. Ito. Part. Yakugaku Zasshi. 1969, Vol. 89, pp. 851-856.

92. Fukushima S, Noro T, Akahori Y, Saiki Y, Ueno A. Studies on the constituents of Leptorumohra miquelianaH. Ito. Part III. The conformation of protofarrerol. Yakugaku Zasshi. 1969, Vol. 89, pp. 1272-1275.

93. Qi-Dong Tu, Ding Li, Yao Sun, Xin-Ya Han, Fan Yi, Yibamu Sha, Yan-Liang Ren, Ming-Wu Ding, Ling-Ling Feng, Jian Wan. Design and syntheses of novel N'-((4-oxo-4Hchromen-3-yl)methylene)benzohydrazide as inhibitors of cyanobacterial fructose-1,6/sedoheptulose-1,7-bisphosphatase. Bioorganic \& Medicinal Chemistry. 2013, Vol. 21 (11), pp. 2826-2831.

94. Tang Y, Fang W, Ma YT, Cai YL, Ruan JL. A novel flavonoid from the root of Macrothelypteris torresiana. Chinese Chem Lett. 20(7), 2009, pp. 815-816.

95. Freitas GC, Batista JM Jr, Franchi GC Jr, Nowill AE, Yamaguchi LF, Vilcachagua JD, Favaro DC, Furlan M, Guimarães EF, Jeffrey CS, Kato MJ. Cytotoxic non-aromatic B-ring flavanones from Piper carniconnectivum C. DC. Phytochemistry. 2014, Vol. 97, pp. 81-7. 
96. G. Szakács, J. P. Annereau, S. Lababidi, U. Shankavaram, A. Arciello, K. J. Bussey, W. Reinhold, Y. Guo, G. D. Kruh, M. Reimers, J. N. Weinstein, M. M. Gottesman. Predicting drug sensitivity and resistance: profiling ABC transporter genes in cancer cells. Cancer Cell. 2004 (2): 129-37. 


\begin{abstract}
Annex

\section{Contents:}

Table A1 - Cytotoxic activity of compounds on the L5178 / L5178 $\mathrm{B} 1$ and MCF-7 / MCF-7 cell line pairs.
\end{abstract}

Table A2 - Cytotoxic activity of selected protoflavones on the A431, A431 $1_{\mathrm{B} 1}$ and $\mathrm{A} 431_{\mathrm{G} 1}$ cell lines.

Table A3 - Cytotoxic activity of selected protoflavones on the MES-SA / MES-SA/Dx5 and KB-3-1 / KB-V1cell line pairs.

Table A4 - Relative inhibition values calculated from calcein uptake assay.

Papers related to the $\mathrm{PhD}$ thesis 
Table A1. Cytotoxic activity of compounds on the L5178 / L5178 $\mathrm{B} 1$ and MCF-7 / MCF-7Dox cell line pairs. A-ring substitutions and $O$-alkyl groups at $\mathrm{C}-1^{\prime}, \mathrm{R}$ and $\mathrm{R}_{1}$ refer to Scheme 2; $\mathrm{SR}=$ selectivity ratio, n.d.: not determined; $\mathrm{n}=3-4$; Dox $=$ doxorubicin .

\begin{tabular}{|c|c|c|c|c|c|c|c|c|}
\hline \multirow{2}{*}{$\begin{array}{c}\text { A-ring } \\
\text { substitution }\end{array}$} & \multirow{2}{*}{$\begin{array}{l}\text { R group } \\
\text { at } C-1^{\prime}\end{array}$} & \multirow[b]{2}{*}{ Compound } & \multicolumn{2}{|c|}{$\mathrm{IC}_{50}(\mu \mathrm{M})$} & \multirow[b]{2}{*}{ SR } & \multicolumn{2}{|c|}{$\mathrm{IC}_{50}(\mu \mathrm{M})$} & \multirow[b]{2}{*}{ SR } \\
\hline & & & L5178 & $\mathrm{L} 5178_{\mathrm{B} 1}$ & & MCF-7 & MCF-7 ${ }_{\text {Dox }}$ & \\
\hline \multirow{9}{*}{$\mathrm{OH}$} & $\mathrm{H}$ & 1 & $0.88 \pm 0.09$ & $0.76 \pm 0.04$ & 1.2 & $5.56 \pm 0.79$ & $1.33 \pm 0.37$ & 4.2 \\
\hline & $\mathrm{Me}$ & 38 & $4.64 \pm 0.43$ & $3.24 \pm 0.68$ & 1.4 & $11.40 \pm 0.31$ & $4.20 \pm 0.13$ & 2.7 \\
\hline & Et & 39 & $5.16 \pm 0.01$ & $5.12 \pm 0.18$ & 1.0 & $6.03 \pm 0.14$ & $3.71 \pm 0.14$ & 1.6 \\
\hline & $\operatorname{Pr}$ & 40 & $6.04 \pm 0.15$ & $4.70 \pm 0.60$ & 1.3 & $6.68 \pm 0.65$ & $2.42 \pm 0.04$ & 2.8 \\
\hline & $i$-Pr & 41 & $10.91 \pm 0.83$ & $8.01 \pm 0.59$ & 1.4 & $>50$ & $>50$ & n.d. \\
\hline & $\mathrm{Bu}$ & 42 & $1.54 \pm 0.15$ & $1.32 \pm 0.12$ & 1.2 & $7.43 \pm 0.10$ & $2.28 \pm 0.03$ & 3.3 \\
\hline & Allyl & 43 & $1.67 \pm 0.12$ & $1.52 \pm 0.02$ & 1.1 & $20.42 \pm 0.76$ & $5.00 \pm 0.11$ & 4.1 \\
\hline & Propargyl & 44 & $2.61 \pm 0.12$ & $2.46 \pm 0.26$ & 1.1 & $8.84 \pm 0.07$ & $2.64 \pm 0.02$ & 3.3 \\
\hline & $\mathrm{Bn}$ & 45 & n.d. & n.d. & & $4.48 \pm 0.42$ & $1.82 \pm 0.20$ & 2.5 \\
\hline \multirow{7}{*}{$\mathrm{OH}_{\mathrm{OH}}$} & $\mathrm{H}$ & 81 & $2.09 \pm 0.06$ & $2.05 \pm 0.03$ & 1.0 & $>50$ & $>50$ & n.d. \\
\hline & $\mathrm{Me}$ & 82 & $0.50 \pm 0.03$ & $0.59 \pm 0.05$ & 0.9 & $>50$ & $>50$ & n.d. \\
\hline & Et & 83 & $1.77 \pm 0.07$ & $1.66 \pm 0.17$ & 1.1 & $20.17 \pm 2.26$ & $4.66 \pm 0.59$ & 4.3 \\
\hline & $\operatorname{Pr}$ & 84 & $1.41 \pm 0.02$ & $1.92 \pm 0.13$ & 0.7 & $9.42 \pm 0.71$ & $2.26 \pm 0.11$ & 4.2 \\
\hline & $\mathrm{Bu}$ & 85 & $3.99 \pm 0.04$ & $4.49 \pm 0.20$ & 0.9 & $27.16 \pm 1.78$ & $6.80 \pm 0.83$ & 4.0 \\
\hline & Allyl & 86 & $1.55 \pm 0.04$ & $0.99 \pm 0.02$ & 1.6 & $10.46 \pm 0.86$ & $1.94 \pm 0.33$ & 5.4 \\
\hline & Propargyl & 87 & $1.53 \pm 0.08$ & $0.96 \pm 0.09$ & 1.6 & $12.30 \pm 1.60$ & $3.81 \pm 0.28$ & 3.2 \\
\hline \multirow[t]{7}{*}{$\mathrm{R}_{1}=\mathrm{Me}$} & $\mathrm{H}$ & 88 & $0.26 \pm 0.02$ & $0.16 \pm 0.02$ & 1.6 & $4.93 \pm 1.27$ & $0.75 \pm 0.06$ & 6.6 \\
\hline & $\mathrm{Me}$ & 89 & $2.27 \pm 0.05$ & $1.45 \pm 0.14$ & 1.6 & $27.12 \pm 2.01$ & $4.58 \pm 0.10$ & 5.9 \\
\hline & Et & 90 & $3.17 \pm 0.26$ & $1.85 \pm 0.16$ & 1.7 & $36.79 \pm 1.17$ & $5.34 \pm 0.10$ & 6.9 \\
\hline & $\operatorname{Pr}$ & 91 & $5.35 \pm 0.23$ & $3.03 \pm 0.17$ & 1.8 & $22.80 \pm 1.40$ & $2.59 \pm 0.05$ & 8.8 \\
\hline & $\mathrm{Bu}$ & 92 & $4.07 \pm 0.10$ & $2.75 \pm 0.14$ & 1.5 & $17.48 \pm 2.23$ & $2.40 \pm 0.09$ & 7.2 \\
\hline & Allyl & 93 & $2.91 \pm 0.13$ & $1.81 \pm 0.09$ & 1.6 & $20.35 \pm 2.07$ & $4.33 \pm 0.45$ & 4.7 \\
\hline & Propargyl & 94 & $1.74 \pm 0.12$ & $0.97 \pm 0.03$ & 1.8 & $19.32 \pm 0.70$ & $4.28 \pm 0.30$ & 4.5 \\
\hline \multirow[t]{14}{*}{$\mathrm{R}_{1}=\mathrm{OMe}$} & $\mathrm{H}$ & 81 & n.d. & n.d. & & $3.92 \pm 0.73$ & $1.05 \pm 0.12$ & 3.7 \\
\hline & $\mathrm{Me}$ & 82 & $18.16 \pm 0.04$ & $11.75 \pm 0.96$ & 1.5 & $32.84 \pm 0.55$ & $12.82 \pm 1.11$ & 2.6 \\
\hline & $\mathrm{Et}$ & 83 & $26.18 \pm 0.28$ & $17.29 \pm 0.48$ & 1.5 & $43.70 \pm 1.91$ & $11.85 \pm 0.34$ & 3.7 \\
\hline & $\mathrm{Bu}$ & 85 & $4.64 \pm 0.01$ & $3.30 \pm 0.26$ & 1.4 & $14.14 \pm 0.27$ & $3.70 \pm 0.06$ & 3.8 \\
\hline & Allyl & 86 & $8.95 \pm 0.04$ & $4.42 \pm 0.38$ & 2.0 & $12.94 \pm 0.06$ & $4.38 \pm 0.09$ & 3.0 \\
\hline & Propargyl & 87 & $9.46 \pm 0.07$ & $8.13 \pm 0.10$ & 1.2 & $21.99 \pm 0.04$ & $7.28 \pm 0.39$ & 3.0 \\
\hline & $\mathrm{H}$ & WYC0209 & $0.15 \pm 0.02$ & $0.13 \pm 0.01$ & $1.2^{a}$ & $2.57 \pm 0.49$ & $1.96 \pm 0.08$ & 1.3 \\
\hline & $\mathrm{Me}$ & 31 & $0.98 \pm 0.08$ & $0.90 \pm 0.11$ & $1.1^{a}$ & $9.18 \pm 0.18$ & $2.69 \pm 0.07$ & 3.4 \\
\hline & Et & 32 & $1.43 \pm 0.17$ & $1.71 \pm 0.11$ & $0.8^{a}$ & $8.25 \pm 0.09$ & $2.39 \pm 0.07$ & 3.5 \\
\hline & $\operatorname{Pr}$ & 33 & $2.34 \pm 0.24$ & $2.05 \pm 0.06$ & $1.1^{a}$ & $8.57 \pm 0.44$ & $1.98 \pm 0.07$ & 4.3 \\
\hline & $\mathrm{iPr}$ & 34 & $11.16 \pm 0.33$ & $9.44 \pm 0.40$ & $1.2^{a}$ & $13.83 \pm 0.63$ & $11.79 \pm 0.75$ & 1.2 \\
\hline & $\mathrm{Bu}$ & 35 & $1.96 \pm 0.11$ & $1.51 \pm 0.14$ & $1.3^{a}$ & $8.73 \pm 0.35$ & $2.80 \pm 0.06$ & 3.1 \\
\hline & Allyl & 36 & $0.97 \pm 0.06$ & $0.85 \pm 0.04$ & $1.1^{a}$ & $13.84 \pm 1.09$ & $1.94 \pm 0.08$ & 7.1 \\
\hline & Propargyl & 37 & $0.94 \pm 0.14$ & $0.88 \pm 0.15$ & $1.1^{a}$ & $10.15 \pm 1.83$ & $2.19 \pm 0.02$ & 4.6 \\
\hline \multirow[t]{6}{*}{$\mathrm{R}_{1}=\mathrm{OEt}$} & $\mathrm{H}$ & 75 & $4.00 \pm 0.03$ & $2.98 \pm 1.03$ & 1.3 & $7.35 \pm 0.45$ & $7.29 \pm 0.08$ & 1.0 \\
\hline & $\mathrm{Me}$ & 76 & $7.58 \pm 0.81$ & $6.60 \pm 0.63$ & 1.1 & $7.27 \pm 0.62$ & $7.87 \pm 1.33$ & 0.9 \\
\hline & Et & 77 & $2.79 \pm 0.32$ & $3.11 \pm 0.42$ & 0.9 & $4.56 \pm 0.25$ & $3.99 \pm 0.02$ & 1.1 \\
\hline & $\operatorname{Pr}$ & 78 & $1.61 \pm 0.12$ & $1.50 \pm 0.34$ & 1.1 & $3.02 \pm 0.30$ & $2.03 \pm 0.05$ & 1.5 \\
\hline & $\mathrm{iPr}$ & 79 & $26.35 \pm 1.48$ & $21.71 \pm 3.69$ & 1.2 & $23.60 \pm 1.26$ & $23.42 \pm 0.76$ & 1.0 \\
\hline & $\mathrm{Bu}$ & 80 & $1.30 \pm 0.11$ & $0.85 \pm 0.26$ & 1.5 & $1.66 \pm 0.24$ & $1.81 \pm 0.02$ & 0.9 \\
\hline $\mathrm{R}_{1}=\mathrm{Et}$ & $\mathrm{H}$ & 72 & $0.85 \pm 0.09$ & $1.06 \pm 0.14$ & 0.8 & $4.35 \pm 0.43$ & $2.68 \pm 0.61$ & 1.6 \\
\hline $\mathrm{R}_{1}=$ Pent & $\mathrm{H}$ & 73 & $0.68 \pm 0.19$ & $1.22 \pm 0.21$ & 0.6 & $1.88 \pm 0.23$ & $0.75 \pm 0.07$ & 2.5 \\
\hline $\mathrm{R}_{1}=\mathrm{Br}$ & $\mathrm{H}$ & 74 & $3.50 \pm 0.20$ & $2.09 \pm 0.07$ & 1.7 & n.d. & n.d. & n.d. \\
\hline \multirow[t]{7}{*}{$\mathrm{R}_{1}=\mathrm{Ph}$} & $\mathrm{H}$ & 66 & $0.98 \pm 0.09$ & $1.14 \pm 0.16$ & 0.9 & $16.20 \pm 0.25$ & $1.23 \pm 0.12$ & 13.1 \\
\hline & $\mathrm{Me}$ & 67 & $4.31 \pm 0.15$ & $4.51 \pm 0.15$ & 1.0 & $23.93 \pm 0.40$ & $2.73 \pm 0.19$ & 8.8 \\
\hline & Et & 68 & $4.62 \pm 0.04$ & $4.78 \pm 0.19$ & 1.0 & $24.41 \pm 0.32$ & $2.97 \pm 0.34$ & 8.2 \\
\hline & $\operatorname{Pr}$ & 69 & $7.50 \pm 0.40$ & $7.64 \pm 0.37$ & 1.0 & $35.16 \pm 0.17$ & $4.61 \pm 0.19$ & 7.6 \\
\hline & $i-\operatorname{Pr}$ & 70 & $13.88 \pm 0.71$ & $13.09 \pm 1.09$ & 1.1 & $30.04 \pm 0.71$ & $7.78 \pm 1.13$ & 3.9 \\
\hline & $\mathrm{Bu}$ & 71 & $5.39 \pm 0.41$ & $6.39 \pm 0.73$ & 0.8 & $33.34 \pm 1.50$ & $4.14 \pm 0.33$ & 8.0 \\
\hline & & Dox & $0.23 \pm 0.01$ & $3.53 \pm 0.44$ & 0.065 & $2.14 \pm 0.30$ & $>20$ & $<0.11$ \\
\hline
\end{tabular}


Table A2. Cytotoxic activity of selected protoflavones on the A431, A431 $1_{\mathrm{B} 1}$ and $\mathrm{A} 431_{\mathrm{G} 1}$ cell lines. A-ring substitutions and $O$-alkyl groups at $\mathrm{C}-1^{\prime}$ are presented, $\mathrm{R}$ and $\mathrm{R}_{1}$ refer to Scheme 2; $\mathrm{SR}=$ selectivity ratio, $\mathrm{n} . \mathrm{d} .:$ not determined; $\mathrm{n}=3-4 ; \mathrm{Mx}=$ mitoxantrone.

\begin{tabular}{|c|c|c|c|c|c|c|c|}
\hline \multirow{2}{*}{$\begin{array}{l}\text { A-ring } \\
\text { substitution }\end{array}$} & \multirow{2}{*}{$\begin{array}{l}\mathrm{R} \text { group } \\
\text { at } \mathrm{C}-1^{\prime}\end{array}$} & & \multicolumn{2}{|c|}{$\mathrm{IC}_{50}(\mu \mathrm{M})$} & \multirow[b]{2}{*}{$\mathrm{SR}_{\mathrm{B} 1}$} & \multicolumn{2}{|l|}{$\mathrm{IC}_{50}(\mu \mathrm{M})$} \\
\hline & & & A431 & $\mathrm{A} 431_{\mathrm{B} 1}$ & & $\mathrm{~A} 431_{\mathrm{G} 2}$ & $\mathrm{SR}_{\mathrm{G} 2}$ \\
\hline & $\mathrm{H}$ & 1 & $2,19 \pm 0.63$ & $2.41 \pm 0.46$ & 0.9 & $9.08 \pm 1.18$ & 0.2 \\
\hline & $\mathrm{Me}$ & 38 & $1.67 \pm 0.34$ & $2.77 \pm 0.56$ & 0.6 & $2.69 \pm 0.33$ & 0.6 \\
\hline & Et & 39 & $1.35 \pm 0.26$ & $1.75 \pm 0.16$ & 0.8 & $2.3 \pm 0.41$ & 0.6 \\
\hline & $\operatorname{Pr}$ & 40 & $0.66 \pm 0.2$ & $0.6 \pm 0.01$ & 1.1 & $1.09 \pm 0.1$ & 0.6 \\
\hline & $i-\operatorname{Pr}$ & 41 & $3.3 \pm 0.93$ & $2.8 \pm 1.91$ & 1.2 & $6.2 \pm 2.53$ & 0.5 \\
\hline & $\mathrm{Bu}$ & 42 & $0.62 \pm 0.14$ & $0.6 \pm 0.18$ & 1.0 & $0.82 \pm 0.05$ & 0.8 \\
\hline & Allyl & 43 & $1.05 \pm 0.35$ & $1.27 \pm 0.18$ & 0.8 & $1.74 \pm 0.32$ & 0.6 \\
\hline & Propargyl & 44 & $1.33 \pm 0.36$ & $1.62 \pm 0.5$ & 0.8 & $2.07 \pm 0.3$ & 0.6 \\
\hline & $\mathrm{Bn}$ & 45 & $0.61 \pm 0.11$ & $0.74 \pm 0.02$ & 0.8 & $0.77 \pm 0.06$ & 0.8 \\
\hline & $\mathrm{H}$ & 81 & $7.27 \pm 2.29$ & $6.23 \pm 1.63$ & 1.2 & $5.00 \pm 0.6$ & 1.5 \\
\hline $\mathrm{R}_{1}=\mathrm{Me}$ & $\mathrm{H}$ & 87 & $2.14 \pm 0.54$ & $2.12 \pm 0.5$ & 1.0 & $1.85 \pm 0.59$ & 1.2 \\
\hline & $\mathrm{H}$ & WYC0209 & $2.24 \pm 0.77$ & $1.99 \pm 0.8$ & 1.1 & $1.68 \pm 0.81$ & 1.3 \\
\hline & $\mathrm{Me}$ & 31 & $0.62 \pm 0.2$ & $0.99 \pm 0.49$ & 0.6 & $0.7 \pm 0.28$ & 0.9 \\
\hline & $\mathrm{Et}$ & 32 & $1.13 \pm 0.41$ & $1.93 \pm 0.77$ & 0.6 & $1.11 \pm 0.33$ & 1.0 \\
\hline & $\operatorname{Pr}$ & 33 & $1.24 \pm 0.38$ & $1.69 \pm 0.2$ & 0.7 & $1.39 \pm 0.19$ & 0.9 \\
\hline & $i-\operatorname{Pr}$ & 34 & $1.29 \pm 0.51$ & $1.77 \pm 0.3$ & 0.7 & $1.36 \pm 0.29$ & 0.9 \\
\hline & $\mathrm{Bu}$ & 35 & $0.91 \pm 0.09$ & $1.33 \pm 0.15$ & 0.7 & $0.96 \pm 0.07$ & 0.9 \\
\hline & Allyl & 36 & $1.45 \pm 0.7$ & $2.14 \pm 0.82$ & 0.7 & $1.3 \pm 0.28$ & 1.1 \\
\hline & Propargyl & 37 & $0.69 \pm 0.21$ & $0.82 \pm 0.24$ & 0.8 & $0.8 \pm 0.08$ & 0.9 \\
\hline $\mathrm{R}_{1}=$ Pent & $\mathrm{H}$ & 73 & $1.16 \pm 0.58$ & $1.35 \pm 0.79$ & 0.9 & $0.98 \pm 0.23$ & 1.2 \\
\hline $\mathrm{R}_{1}=\mathrm{Ph}$ & $\mathrm{H}$ & 66 & $2.5 \pm 0.73$ & $2.58 \pm 0.27$ & 1.0 & $2.23 \pm 0.8$ & 1.1 \\
\hline & $\mathrm{Me}$ & 67 & $1.66 \pm 0.12$ & $2.06 \pm 0.19$ & 0.8 & $1.54 \pm 0.26$ & 1.1 \\
\hline & $\mathrm{Et}$ & 68 & $2.15 \pm 0.23$ & $3.22 \pm 0.23$ & 0.7 & $2.08 \pm 0.48$ & 1.0 \\
\hline & $\operatorname{Pr}$ & 69 & $2.63 \pm 0.39$ & $3.32 \pm 0.13$ & 0.8 & $2.46 \pm 0.1$ & 1.1 \\
\hline & iPr & 70 & $4.31 \pm 0.61$ & $5.4 \pm 1.1$ & 0.8 & $5.3 \pm 0.58$ & 0.8 \\
\hline & $\mathrm{Bu}$ & 71 & $2.28 \pm 0.19$ & $3.27 \pm 0.05$ & 0.7 & $2.14 \pm 0.02$ & 1.1 \\
\hline & & $\mathrm{Mx}$ & $1.22 \pm 0.62$ & $5.65 \pm 0.57$ & 0.2 & $6.15 \pm 0.63$ & 0.2 \\
\hline
\end{tabular}


Table A3. Cytotoxic activity of selected protoflavones on the MES-SA / MES-SA/Dx5 and KB-3-1 / KB-V1cell line pairs. A-ring substitutions and $O$-alkyl groups at C-1' are presented, $\mathrm{R}$ and $\mathrm{R}_{1}$ refer to Scheme 2; $\mathrm{SR}=$ selectivity ratio, n.d.: not determined; $\mathrm{n}=3-4$; Dox $=$ doxorubicin, $\mathrm{Vbl}=$ vinblastine.

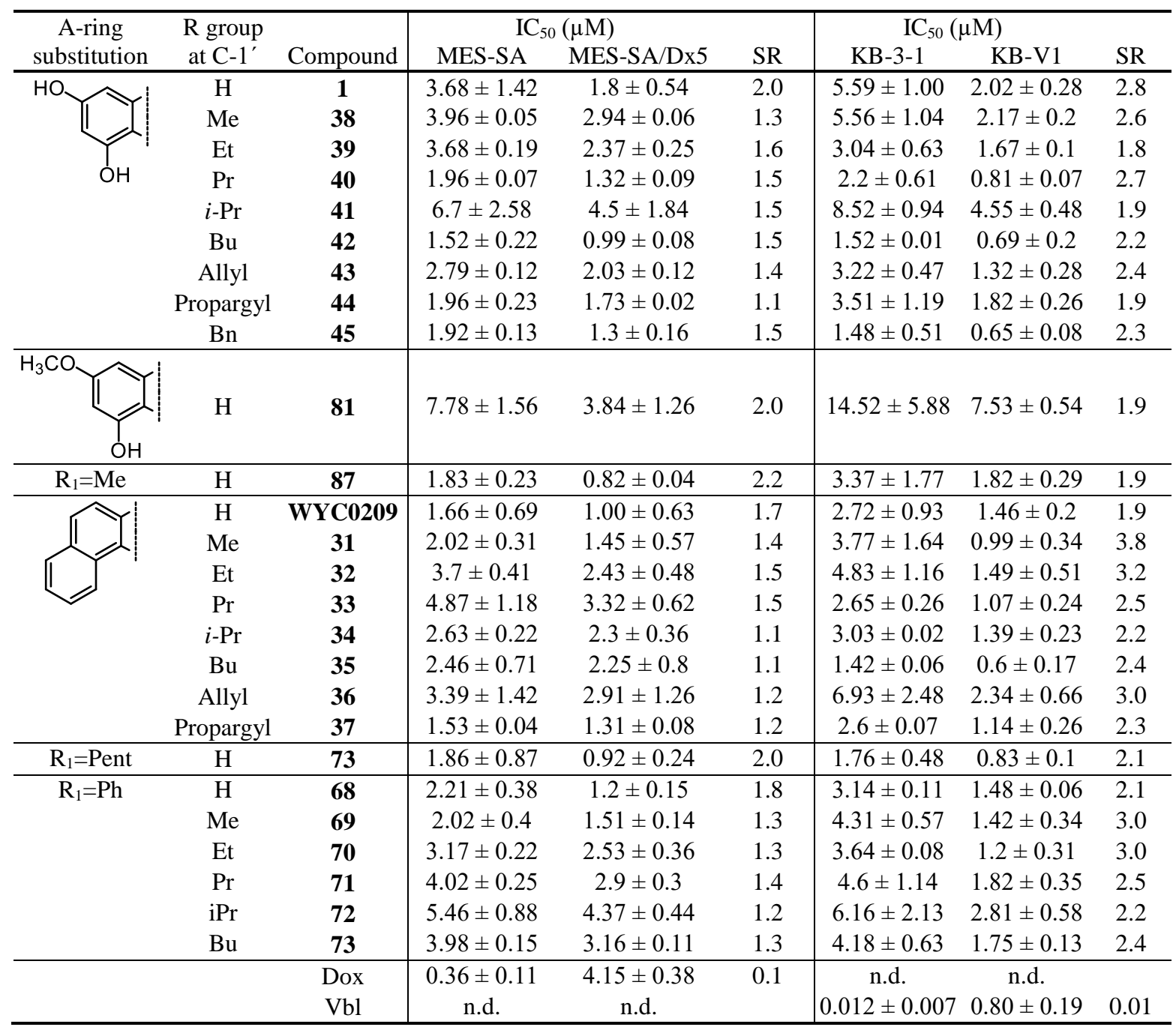


Table A4. Relative inhibition values calculated from the calcein uptake assay.

\begin{tabular}{ccc}
\hline Compound & \multicolumn{2}{c}{ Relative inhibition } \\
& $2 \mu \mathrm{M}$ & $20 \mu \mathrm{M}$ \\
\hline $\mathbf{6 9}$ & $1 \%$ & $3 \%$ \\
$\mathbf{8 8}$ & $6 \%$ & $7 \%$ \\
$\mathbf{7 7}$ & $13 \%$ & $7 \%$ \\
$\mathbf{6 6}$ & $7 \%$ & $45 \%$ \\
WYC0209 & $8 \%$ & $11 \%$ \\
$\mathbf{8 1}$ & $1 \%$ & $1 \%$ \\
$\mathbf{1}$ & $4 \%$ & $7 \%$ \\
$\mathbf{7 3}$ & $1 \%$ & $14 \%$ \\
$\mathbf{7 4}$ & $2 \%$ & $27 \%$ \\
$\mathbf{6 6}$ & $3 \%$ & $32 \%$ \\
$\mathbf{6 7}$ & $2 \%$ & $47 \%$ \\
$\mathbf{6 8}$ & $5 \%$ & $36 \%$ \\
$\mathbf{3 1}$ & $1 \%$ & $7 \%$ \\
$\mathbf{3 2}$ & $1 \%$ & $6 \%$ \\
$\mathbf{3 3}$ & $0 \%$ & $6 \%$ \\
$\mathbf{3 4}$ & $0 \%$ & $13 \%$ \\
$\mathbf{3 6}$ & $0 \%$ & $25 \%$ \\
$\mathbf{3 7}$ & $1 \%$ & $28 \%$ \\
Verapamil & & $100 \%$ \\
\hline
\end{tabular}




\title{
Direct Semi-Synthesis of the Anticancer Lead-Drug Protoapigenone from Apigenin, and Synthesis of Further New Cytotoxic Protoflavone Derivatives
}

\author{
Attila Hunyadi ${ }^{1,29}$, Da-Wei Chuang ${ }^{29}$, Balazs Danko ${ }^{1}$, Michael Y. Chiang ${ }^{3}$, Chia-Lin Lee ${ }^{4}$, Hui-Chun \\ Wang $^{2}$, Chin-Chung $\mathrm{Wu}^{2}$, Fang-Rong Chang ${ }^{2,5,6 *}$, Yang-Chang $\mathrm{Wu}^{2,4,7 *}$
}

1 Institute of Pharmacognosy, University of Szeged, Szeged, Hungary, 2 Graduate Institute of Natural Products, Kaohsiung Medical University, Kaohsiung, Taiwan, 3 Department of Chemistry, National Sun Yat-Sen University, Kaohsiung, Taiwan, 4 Graduate Institute of Integrated Medicine, College of Chinese Medicine, China Medical University, Taichung, Taiwan, $\mathbf{5}$ Cancer Center, Kaohsiung Medical University Hospital, Kaohsiung, Taiwan, 6 Research and Development Center of Chinese Herbal Medicines and New Drugs, College of Pharmacy, Kaohsiung Medical University, Kaohsiung, Taiwan, 7 Natural Medicinal Products Research Center, China Medical University Hospital, Taichung, Taiwan

\begin{abstract}
Protoapigenone, a natural flavonoid possessing an unusual p-quinol moiety on its B-ring, is a novel prospective anticancer agent with low toxicity that is currently in development. The first economical, one-step synthesis of protoapigenone from apigenin is described on up to gram scale. 13 new 1 '-O-alkylflavone analogs were also synthesized, either from apigenin or $\beta$-naphthoflavone. The in vitro cytotoxic activity of each compound was tested on six human cancer cell lines (HepG2, Hep3B, Ca9-22, A549, MCF-7 and MDA-MB-231). In the case of 1'-O-alkyl-protoapigenone derivatives, structure-activity relationships were found depending on the side-chain, and protoapigenone 1'-O-butyl ether was found to exert significantly stronger activity against three of the cell lines (Hep3B, MCF-7 and MDA-MB-231) than its non-substituted analog, protoapigenone itself. In contrast to this, all $\beta$-naphthoflavone derivatives bearing the same pharmacophore on their B-ring showed decreased cytotoxic activities when substituted with an $O$-alkyl side-chain at position $1^{\prime}$, comparing to that of the non-substituted compound.
\end{abstract}

Citation: Hunyadi A, Chuang D-W, Danko B, Chiang MY, Lee C-L, et al. (2011) Direct Semi-Synthesis of the Anticancer Lead-Drug Protoapigenone from Apigenin, and Synthesis of Further New Cytotoxic Protoflavone Derivatives. PLoS ONE 6(8): e23922. doi:10.1371/journal.pone.0023922

Editor: Matthew H. Todd, University of Sydney, Australia

Received November 5, 2010; Accepted July 31, 2011; Published August 30, 2011

Copyright: (c) 2011 Hunyadi et al. This is an open-access article distributed under the terms of the Creative Commons Attribution License, which permits unrestricted use, distribution, and reproduction in any medium, provided the original author and source are credited.

Funding: This work was supported by the Department of Health, Executive Yuan, Taiwan (DOH99-TD-C-111-002), the National Science Council of Taiwan, the National Sun Yat-Sen University-Kaohsiung Medical University Joint Research Center for YCW, the New Hungary Development Plan (TÁMOP-4.2.2-08/1-20080013), and by the Zoltán Magyary postdoctoral fellowship (EEA and Norway Grants, 104/2009) for AH. The funders had no role in study design, data collection and analysis, decision to publish, or preparation of the manuscript.

Competing Interests: The authors have declared that no competing interests exist.

*E-mail: aaronfrc@kmu.edu.tw (FRC); yachwu@mail.cmu.edu.tw (YCW)

9 These authors contributed equally to this work.

\section{Introduction}

Flavonoids are among the most widespread secondary plant metabolites and play a significant role in the prevention of several chronic diseases, including cancer [1-3]. Due to their numerous anticancer functions, including antiproliferative, cell cycle arresting and pro-apoptotic effects, certain members of this chemical group are believed to have potential not only in chemoprevention but also as future chemotherapy agents for treating cancer $[4,5]$.

Protoapigenone (1) was first isolated by our group from the Formosan fern Thelypteris torresiana [6]. This compound has a close structural and likely biosynthetic relationship with apigenin (2), a common 4'-hydroxy-flavone (Figure 1) that is abundantly present in fruits and vegetables and is considered a particularly potent cancer chemopreventive flavonoid, according to a growing body of data $[7,8]$.

In vitro, $\mathbf{1}$ exhibits significant antitumor activities toward HepG2, Hep3B, MCF-7, A549 and MDA-MB-231 tumor cell lines IC $_{50}$ $0.27-3.88 \mu \mathrm{g} / \mathrm{mL}$ ). The compound was also found to induce apoptosis in human prostate cancer cells [9] and to selectively inhibit ovarian cancer cell growth both in vitro and in vivo in nude mice while causing no major side effects in the latter case [10]. In a recent study, 1 was shown to exert dose-dependent DNA damage, apoptosis, and $\mathrm{G} 2 / \mathrm{M}$ arrest at micromolar concentrations in a lung cancer cell line (H1299) [11]. Based on its potent proapoptotic activity and apparent low toxicity, $\mathbf{1}$ was chosen by the National Science Council of Taiwan as the lead compound for the development of a new class of anticancer drugs. Presently, in vivo toxicology studies are being performed as part of the preclinical phase, which have created a need for an economical way to drastically increase the production of $\mathbf{1}$.<smiles>O=C1C=CC(O)(c2cc(=O)c3c(O)cc(O)cc3o2)C=C1</smiles><smiles>O=c1cc(-c2ccc(O)cc2)oc2cc(O)cc(O)c12</smiles>

Figure 1. Structures of protoapigenone (1) and apigenin (2). doi:10.1371/journal.pone.0023922.g001 
In 2007, a six-step total synthesis of $\mathbf{1}$ was reported by our group [12]. After difficulties in carrying out the direct semisynthesis of $\mathbf{1}$ from 2, we used methoxymethyl-diprotected trihydroxyacetophenones and 4-benzyloxybenzaldehyde as starting materials to prevent the A-ring of $\mathbf{2}$ from oxidation, and we applied a hypervalent iodine reaction to the 7-protected apigenin skeleton in the second-to-last step to obtain the $p$-quinol moiety on the B-ring. Finally, 1 was successfully obtained after the removal of the protecting group. To carry out the key oxidation step that formed the pharmacophore on the B-ring, we applied 2 equivalent (eq.) of [bis(trifluoroacetoxy)iodo]benzene (commonly referred to as PIFA) in the presence of 0.2 eq. of TEMPO, a commercially available free radical catalyst. Although in some related oxidative dearomatization reactions phenyliodine diacetate (PIDA) was found to give higher yield of product as compared to when PIFA was used [13], PIDA was less effective in our case. This was also in accordance with the results of Wells et al. [14], who found the use of PIFA and TEMPO advantegous over PIDA. Based on these, PIFA was kept as the only oxidizing agent when the methodology reported here was developed. The reaction was originally performed by stirring at $25^{\circ} \mathrm{C}$ for $90 \mathrm{~min}$ in acetonitrile:water (9:1, v/v) at a concentration of $18 \mathrm{mg} / \mathrm{mL}$ of 7-protected 2, and, after deprotection, 1 was successfully obtained. Figure 2 shows an outline of this procedure, including reaction times and isolated yields of the reaction steps.

The procedure resulted in the synthesis of protoapigenone as well as several of its derivatives which allowed SAR data to be gathered. However, due to the long reaction times and the slow chromatographic purifications, the procedure took approximately one to two months to complete, and resulted in only a $3.3 \%$ overall yield of $\mathbf{1}$. Because this means of production was unable to satisfy the high material needs for the extensive preclinical pharmacological investigation, the improvement of the synthetic route has become an urgent need and a primary objective of our research program.

\section{Results and Discussion}

\section{Synthesis}

Our attempt to improve the yield of the original method began with the optimization of the oxidation of 7-MOM-apigenin but led to the surprising observation that with appropriate reaction conditions, $\mathbf{1}$ could be directly synthesized from $\mathbf{2}$ without the need for protecting groups. Optimization of the direct semisynthesis provided the following insights:
- By decreasing the concentration of the starting material to $1 \mathrm{mg} / \mathrm{mL}$, the yield could be improved significantly; further decreases of concentration did not provide further increases in the yield.

- TEMPO significantly decreases the yield, and the formation of $\mathbf{1}$ may be prevented completely by increasing the amount of TEMPO used. Thus, it should be omitted from the reaction.

- The reaction is highly dependent on the solvent used. For success, the majority of the solvent should be a nonnucleophilic polar solvent, preferably acetonitrile. When using acetone:water $(9: 1, \mathrm{v} / \mathrm{v})$ and THF:water $(9: 1, \mathrm{v} / \mathrm{v})$, the reaction failed. EtOAc saturated with water gave traces of $\mathbf{1}$, while 1,1,1,3,3,3-hexafluoro-isopropanol:water $(9: 1, \mathrm{v} / \mathrm{v})$ resulted in about half the yield of that obtained using the ACN:water mixture.

- Low energy microwave heating $\left(70^{\circ} \mathrm{C}, 1 \mathrm{~min}, 500 \mathrm{~W}\right)$ can increase the yield by more than two-fold, compared to when the reaction is performed at room temperature.

- The TFA (trifluoroacetic acid) derived from the PIFA in the reaction significantly decreases the $\mathrm{pH}$. Compound $\mathbf{1}$ does not suffer quick decomposition, but it is slightly sensitive to acidic environments, so it is recommended that it be purified as soon as possible. On the other hand, neutralizing the TFA is not favorable, as the remaining ionizable phenolic hydroxyl groups may lead to a less complete separation at the purification process.

As mentioned above, we performed the reaction via a quick, microwave-assisted oxidation of $\mathbf{2}(1 \mathrm{mg} / \mathrm{mL}$ in acetonitrile:water 9:1, v/v) with 2 eq. of PIFA, which resulted in a $31 \%$ isolated yield for $100 \mathrm{mg}$ of starting material. This was a great improvement over the previous method [12]. The purification consisted of a solid phase extraction followed by gel chromatography on Sephadex LH-20, which also resulted in the isolation of two side-products, $\mathbf{1}^{\prime}$ and $\mathbf{1}^{\prime \prime}$. Based on its NMR spectra and chromatographic properties in comparison with available testcompound and literature data, $\mathbf{1}^{\prime}$ was identified as luteolin, a common flavone and an expected side-product of the performed reaction [15]. By means of ESI-MS and HRMS data, $\mathbf{1}^{\prime \prime}$ was found to have a molecular mass of 554 and a constitution of $\mathrm{C}_{30} \mathrm{H}_{18} \mathrm{O}_{11}$ corresponding to an apigenin-protoapigenone dimer. The ${ }^{1} \mathrm{H}$ NMR spectrum suggested, that a ring corresponding to the B-ring of apigenin is present $(2 \mathrm{H}$ at both 7.38 and $6.77 \mathrm{ppm}, \mathcal{f}=8.8 \mathrm{~Hz})$. Interestingly, four separate hydrogens were shown as doublet-

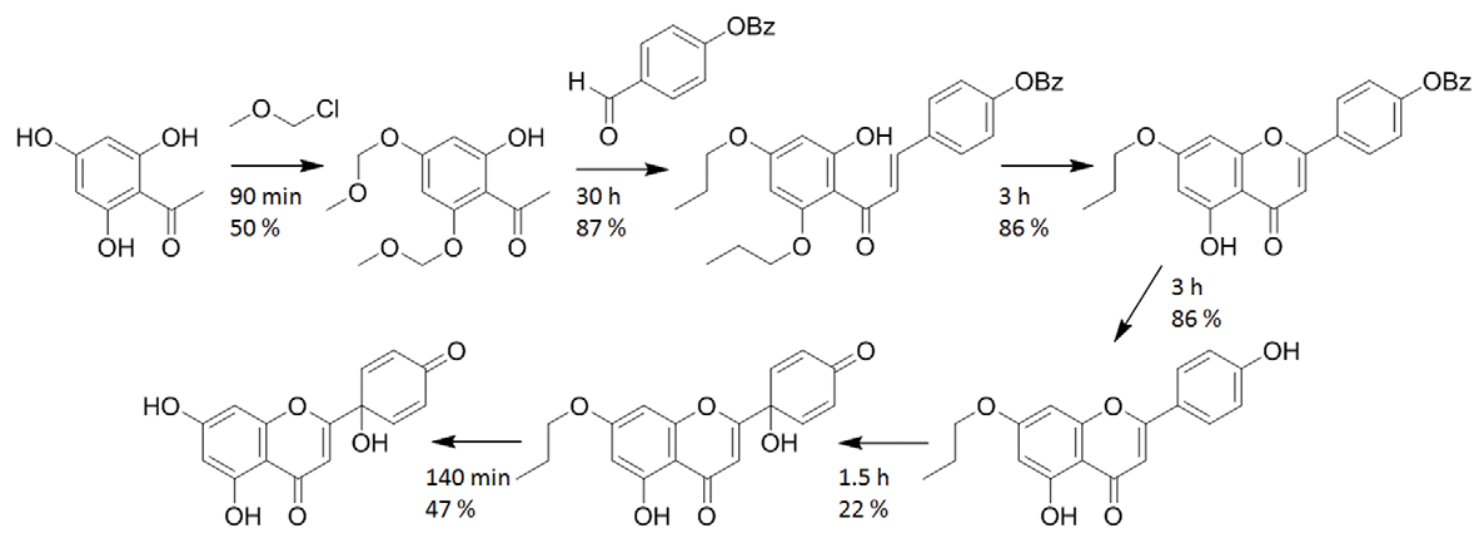

Figure 2. The total-synthesis of protoapigenone as reported previously [12]. Reaction times and isolated yields are shown for each reaction step. Purification of each intermediate product was performed by column chromatography on silica. doi:10.1371/journal.pone.0023922.g002 
doublets with the larger coupling constant of $10.0 \mathrm{~Hz}$. This coupling would be characteristic to the two doublets, two hydrogens each, of the B-ring of protoapigenone. Based on the ${ }^{1} \mathrm{H}-{ }^{1} \mathrm{H}$ COSY spectrum, these four hydrogens were also on the same ring, suggesting a cyclohexadienone ring, as in protoapigenone, also to be present. Differences between the pairs of corresponding protons on this ring are suggested to arise from the effect of the close aromatic B-ring of the apigenin part, which was also shown by NOESY correlations between hydrogens of these two rings. The number of non-OH hydrogens and the presence of only two meta-coupling doublets accompanied by two singlets in the ${ }^{1} \mathrm{H}$ spectrum suggested a $\mathrm{C}-\mathrm{C}$ bond between parts of the dimer. Further assignments were made based on the HMQC and HMBC spectra, and an 8-3" junction was suggested. Structure, numbering and key HMBC and NOESY correlations of $\mathbf{1}^{\prime \prime}$ are shown in Figure 3.

Hypervalent iodine reagents are widely used for the oxidative dearomatization of 4-substituted phenols in the presence of an appropriate nucleophile to obtain 4,4-disubstituted cyclohexadienones [15-16], and reaction mechanisms involving carbocation [13] and/or cation-radical [17] intermediates have been suggested in the literature. Considering the structures of our isolated products we suggest the mechanism shown in Figure 4.

The presence of $\mathbf{1}^{\prime \prime}$ as a side-product also provides a possible explanation for the failure of the previous conditions, which most likely failed due to the much higher concentration of starting material and thus the more favorable dimerization or even oligomer formation via C-C coupling side-reactions. Considering the large variety of other side-reactions also available in this system, the $31 \%$ yield of $\mathbf{1}$ shows an unexpectedly good selectivity of PIFA for the 4'-OH group. This selectivity may arise from two phenomena: (1) the greater acidity of the $7-\mathrm{OH}$ group compared to that of the $4^{\prime}-\mathrm{OH}$ group (in a mixture of $\mathrm{MeOH}$ :water $(1: 2, \mathrm{v} / \mathrm{v}$ ) $\mathrm{p} K_{\mathrm{a}}=6.9$ and 8.6, respectively [18]) causes 2 to mostly exist in the 7-mono-anionic form at the neutral $\mathrm{pH}$ of the reaction's start, and (2) the intramolecular hydrogen-bond to the 5-OH group makes it more stable than the $4^{\prime}-\mathrm{OH}$.

According to our main purpose of establishing a useful method for larger scale production of $\mathbf{1}$, we scaled-up the procedure by oxidizing $800.0 \mathrm{mg}, 2.0 \mathrm{~g}$ and $5.0 \mathrm{~g}$ of 2, which provided $251.3 \mathrm{mg}(29.6 \%), 546.2 \mathrm{mg}(25.8 \%)$ and $1.18 \mathrm{~g}(22.3 \%)$ of $\mathbf{1}$, respectively (see Materials and Methods section). Due to the large volumes of solvent required (in order to prevent excessive sidereactions, as mentioned above), further increases in the amount of starting material used would have been difficult under laboratory conditions. On the other hand, the solvent volume increase was the most probable reason for the slight decrease of the yield on larger scales, because it resulted in slower cooling after the reaction was finished and a much longer evaporation process. This level of productivity should, however, be suitable to obtain appropriate amounts of $\mathbf{1}$ for the remaining preclinical studies.

The optimized reaction was also performed on $50.0 \mathrm{mg}$ of $\beta$ naphthoflavone $(\mathbf{1 0})$, which served as the basis for the most active synthetic derivative to date. This reaction yielded a remarkable $59 \%$ of the corresponding analog $(\mathbf{1 1})$, compared to the $16 \%$ yield reported previously [12].

For both starting materials $\mathbf{2}$ and 10, further derivatives were also synthesized by replacing water with various alcohols to obtain the corresponding 1'-O-alkyl ethers, as shown in Figure 5.

\section{In vitro activity}

The cytotoxic activities of all protoapigenone analogs obtained were tested on six human cancer cell lines, including HepG2 and Hep3B (hepatic), Ca9-22 (oral), A549 (lung), as well as MCF-7 and MDA-MB-231 (breast) cancer cells, with doxorubicin as a positive control. $\mathrm{IC}_{50}$ values and $95 \%$ confidence intervals of the best fit values obtained by nonlinear regression are presented in Table 1.

To elaborate on the findings of the previous investigation, which showed that $1^{\prime}$-O-methyl substitution decreases the activity of 1 [12], the present results demonstrated that increasing the length of a 1 '-O-alkyl side-chain on the protoapigenone skeletone typically increases the activity comparing to that of the methylsubstituted derivative (compound $\mathbf{3}$ ). As a result of this tendency, several analogs were found to exert practically the same, or, as in case of the butyl-substituted compound 7 against the Hep3B, MCF-7 and MDA-MB-231 cell lines, even higher in vitro cytotoxic activities comparing to that of the non-substituted compound 1. On the other hand, a 2" to $3^{\prime \prime}$ unsaturated sidechain (allyl, propargyl derivatives; $\mathbf{8}, \mathbf{9}$ ) decreased the activity on the A549 cell line comparing to that of compound $\mathbf{5}$ bearing a saturated propyl side-chain $(\mathrm{p}<0.05$ and 0.001 , respectively). In addition to this, presence of a branching side-chain (isopropyl derivative; 6) strongly decreased the activity on each cell line as compared to that of $\mathbf{5}(\mathrm{p}<0.01$ in case of Ca9-22 and $\mathrm{p}<0.001$ in case of all other cell lines), resulting in low activities comparable to those of the methyl-substituted compound 3. Figure 6 shows the activities of all protoapigenone analogs on the Hep3B (A), MCF-7 (B), and MDA-MB-231 (C) cell lines, where 7 was found to exert significantly stronger activity than that of the lead compound $\mathbf{1}$.

Interestingly, the activity of the $\beta$-naphthoflavone derivatives (12-18) does not seem to follow the same trend, except for the effect of the side-chain branching (isopropyl; 15, compared to the
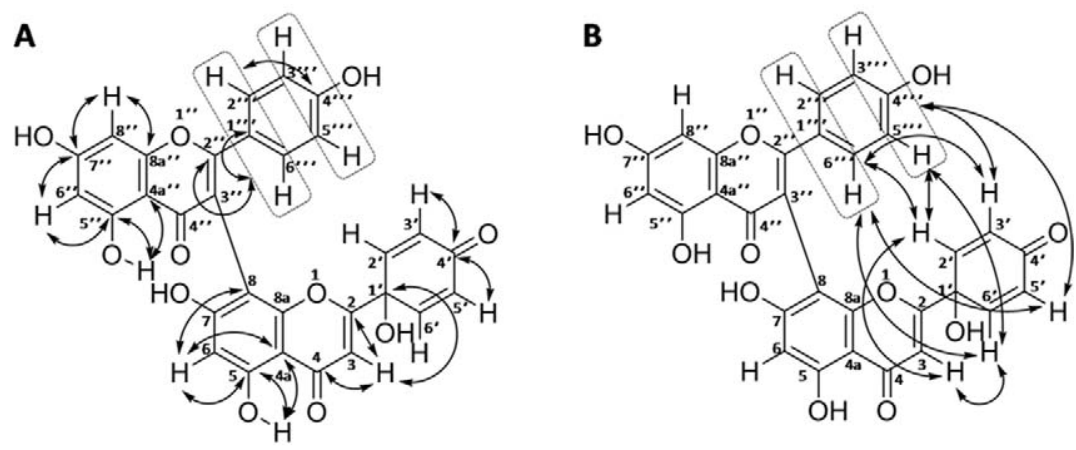

Figure 3. Structure, numbering and key HMBC (A) and NOESY (B) correlations of 1". doi:10.1371/journal.pone.0023922.g003 


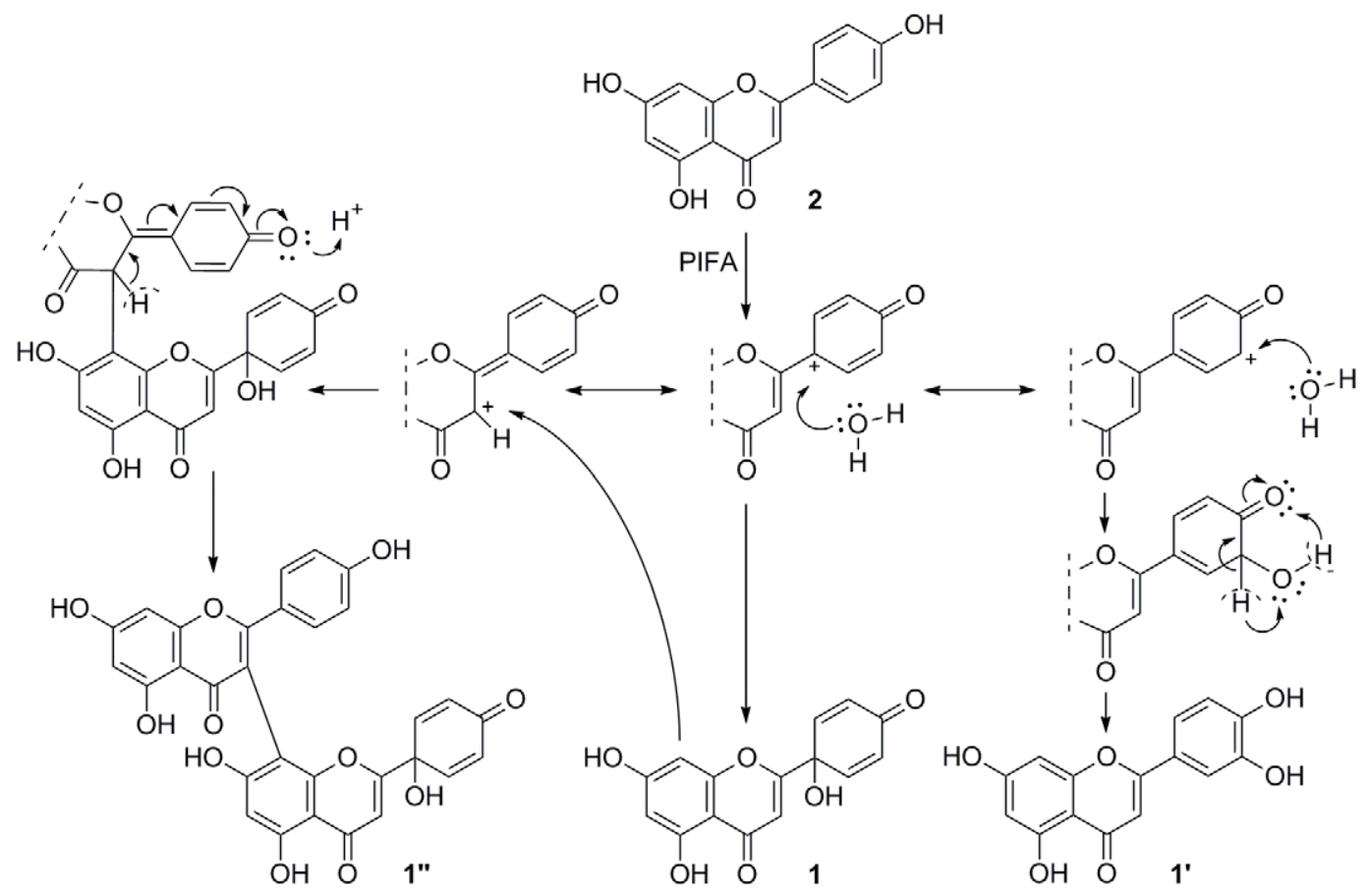

Figure 4. Suggested reaction mechanism for the synthesis of 1 from 2 and product structures obtained. doi:10.1371/journal.pone.0023922.g004

propyl; 14) on most of the cell lines with the exception of A-549, and all of these derivatives showed a marked decrease in the activity as compared to that of $\mathbf{1 1}$.

These results may underline the importance of lipophilicity in the activity of these compounds, which would also explain why derivatives of the more lipophilic $\beta$-naphthoflavone as compared to those of apigenin did not show a similar increase in activity when length of the lipophilic side-chain was increased.

The fact, that a $1^{\prime}-O$ substitution of protoapigenone by a longer, 3 to 4 carbon aliphatic side-chain can restore, or, in case of some cell lines, even increase the in vitro activity comparing to that of the non-substituted compound, makes these derivatives, and particularly compound $\mathbf{7}$, very interesting new candidates for in vivo studies. Not only their increased lipophilicity will result in significantly different pharmacokinetical properties and consequential activity change, but we can also assume, that the $1^{\prime}-O-$ alkylation may increase the stability of the pharmacophore B-ring by protecting it from decomposition and/or metabolic conversion via re-aromatization to the original flavone, apigenin in this case.

\section{Conclusions}

Our results can be summarized as the following:

1. The first economical method to obtain gram scale amounts of protoapigenone was developed via its direct semisynthesis from apigenin. This achievement is a great step forward for research on this promising anticancer compound, considering the need for large amounts of material to carry out toxicological studies.

2. Several new $1^{\prime}$-O-alkyl-protoapigenone as well as the corresponding $\beta$-naphthoflavone derivatives were synthesized. The in vitro cytotoxicity assay of these compounds revealed, that in case of the protoapigenone derivatives a longer aliphatic side-
A

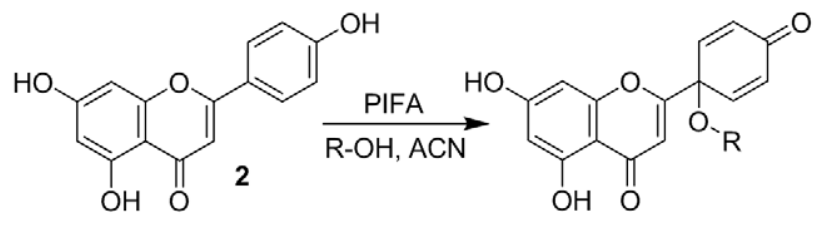

B<smiles>[R]OC1(c2cc(=O)c3c(ccc4ccccc43)o2)C=CC(=O)C=C1C([R])[R]</smiles>

\section{R Compound}

$\begin{array}{ll}\text { H } & \mathbf{1} \\ \text { Methyl } & \mathbf{3} \\ \text { Ethyl } & \mathbf{4} \\ \text { Propyl } & \mathbf{5} \\ \text { Isopropyl } & \mathbf{6} \\ \text { Butyl } & \mathbf{7} \\ \text { Allyl } & \mathbf{8} \\ \text { Propargyl } & \mathbf{9} \\ \text { H } & \\ \text { Methyl } & 11 \\ \text { Ethyl } & 13 \\ \text { Propyl } & 14 \\ \text { Isopropyl } & 15 \\ \text { Butyl } & 16 \\ \text { Allyl } & 17 \\ \text { Propargyl } & 18\end{array}$

Figure 5. The reaction of $\mathbf{2}$ and 10 with PIFA in presence of water or various alcohols. doi:10.1371/journal.pone.0023922.g005 
Table 1. In vitro cytotoxic activities of the compounds obtained.

\begin{tabular}{|c|c|c|c|c|c|c|}
\hline \multirow[t]{2}{*}{ Compound } & \multicolumn{6}{|l|}{$\mathrm{IC}_{50}(\mu \mathrm{M})$} \\
\hline & HepG2 & Нер3В & Ca9-22 & A549 & MCF-7 & MDA-MB-231 \\
\hline 1 & $3.07(2.83-3.34)$ & $1.21(1.19-1.24)$ & $0.780 .49-1.24)$ & $11.29(10.07-12.65)$ & $1.70(1.58-1.82)$ & $1.35(1.17-1.55)$ \\
\hline 1" & $\sim 19.73$ & $>36$ & $16.89(14.58-19.57)$ & $>36$ & $>36$ & $\sim 18.38$ \\
\hline 3 & $8.99(6.12-13.21)$ & $3.36(3.19-3.54)$ & $2.021 .64-2.49)$ & $21.78(20.41-23.24)$ & $6.76(6.07-7.53)$ & $2.82(2.61-3.04)$ \\
\hline 4 & $8.27(8.03-8.51)$ & $1.73(1.68-1.78)$ & $1.00(0.81-1.24)$ & $13.45(12.01-15.07)$ & $3.15(2.23-4.45)$ & $1.57(1.45-1.71)$ \\
\hline 5 & $\sim 6.90$ & $1.66(1.65-1.68)$ & $0.81(0.80-0.82)$ & $12.39(11.04-13.91)$ & $2.36(2.06-2.69)$ & $1.26(1.04-1.53)$ \\
\hline 6 & $\sim 19.70$ & $3.59(3.08-4.19)$ & $1.80(0.89-3.65)$ & $20.06(16.22-24.80)$ & $11.43(10.81-12.09)$ & $2.30(2.06-2.56)$ \\
\hline 7 & $8.78(8.10-9.51)$ & $\mathbf{0 . 8 2}^{(*)}(0.80-0.83)$ & $\sim 0.70$ & $10.76(9.25-12.51)$ & $\mathbf{1 . 3 8 ^ { ( * ) }}(1.32-1.44)$ & $\mathbf{0 . 6 2} 2^{(* * *)}(0.47-0.81)$ \\
\hline 8 & $7.92(7.74-8.10)$ & $1.61(1.54-1.67)$ & $0.93(0.88-0.98)$ & $17.02(16.14-17.94)$ & $2.47(2.26-2.70)$ & $1.53(1.41-1.66)$ \\
\hline 9 & $\sim 7.81$ & $1.20(1.19-1.21)$ & $0.89(0.83-0.96)$ & $20.35(17.33-23.91)$ & $2.32(2.14-2.54)$ & $1.75(1.58-1.93)$ \\
\hline 11 & $1.06(1.04-1.08)$ & $0.25(0.20-0.30)$ & $0.23(0.16-0.33)$ & $2.19(2.03-2.37)$ & $\sim 0.61$ & $0.43(0.41-0.45)$ \\
\hline 12 & $19.48(17.02-22.30)$ & $0.98(0.90-1.06)$ & $0.82(0.73-0.92)$ & $23.17(20.91-25.68)$ & $2.03(1.78-2.32)$ & $1.32(1.18-1.46)$ \\
\hline 13 & $9.96(8.64-11.49)$ & $0.85(0.83-0.86)$ & $1.03(0.94-1.21)$ & $17.88(15.26-20.95)$ & $3.45(3.10-3.83)$ & $1.68(1.55-1.81)$ \\
\hline 14 & $10.53(8.68-12.77)$ & $0.88(0.85-0.91)$ & $0.79(0.77-0.81)$ & $21.72(16.81-28.07)$ & $2.92(2.49-3.44)$ & $1.46(1.34-1.59)$ \\
\hline 15 & $19.21(17.00-21.71)$ & $2.48(1.98-3.11)$ & $2.18(1.73-2.73)$ & $20.66(18.00-23.72)$ & $2.33(2.10-2.59)$ & $2.97(2.66-3.31)$ \\
\hline 16 & $7.96(7.85-8.06)$ & $0.940 .85-1.03)$ & $1.01(0.89-1.15)$ & $15.66(14.90-16.45)$ & $3.05(2.50-3.72)$ & $1.70(1.56-1.84)$ \\
\hline 17 & $9.43(9.32-9.55)$ & $0.85(0.81-0.90)$ & $0.83(0.81-0.86)$ & $15.38(14.00-16.90)$ & $2.28(2.14-2.43)$ & $1.46(1.37-1.56)$ \\
\hline 18 & $8.14(7.82-8.47)$ & $0.88(0.73-1.05)$ & $0.86(0.85-0.87)$ & $19.30(17.10-21.79)$ & $2.24(2.10-2.40)$ & $1.89(1.68-2.12)$ \\
\hline D & $0.830 .49-1.39$ & $1.14(0.78-1.69)$ & $0.60(0.47-0.76)$ & $\sim 1.86$ & $1.24(0.76-2.00)$ & $\sim 1.73$ \\
\hline
\end{tabular}

Individual $\mathrm{IC}_{50}$ values were compared to those of $\mathbf{1}$ (in case of 3-9) or $\mathbf{1 1}$ (in case of 12-18) by one way ANOVA and Dunnet's Multiple Comparison Test and by unpaired T-tests in case of compounds $\mathbf{7}$ and $\mathbf{1}$ (Hep3B and MCF7 only). Significantly stronger activities than that of the reference compound are only marked. $n=3$; *: $p<0.05$ by unpaired T-test, while variances were not significantly different;

$* 0.001$ by ANOVA;

$\sim$ : ambiguous fitting, very wide confidence intervals; $\mathbf{D}$ : doxorubicin

doi:10.1371/journal.pone.0023922.t001

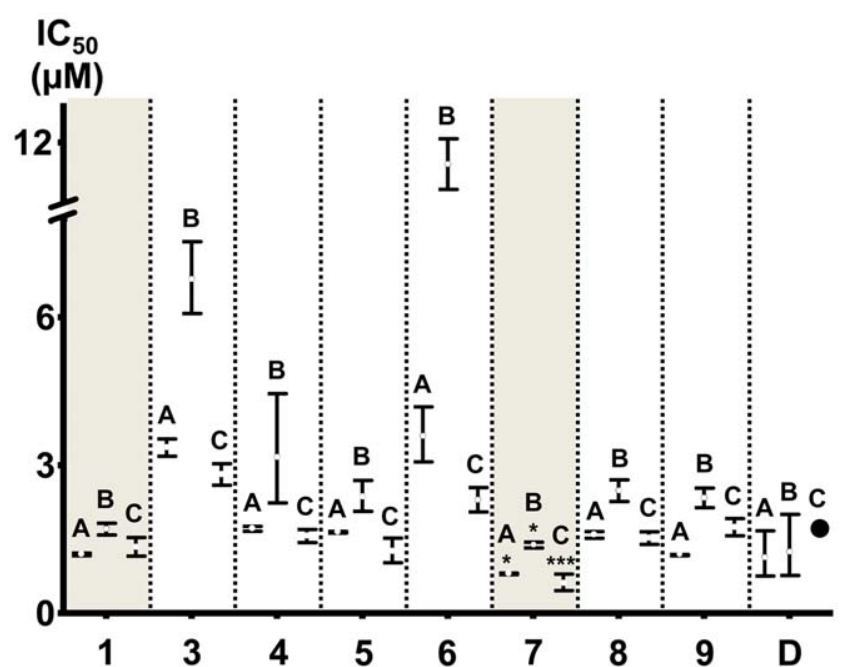

Figure 6. In vitro cytotoxic activities of the protoapigenone analogs against the Hep3B, MCF-7 and MDA-MB-231 cell lines. Numbers on the abscissa represent the corresponding compounds, while charts A, B and C represent activities against Hep3B, MCF-7 and MDA-MB-231 cell lines, respectively. Error bars represent the $95 \%$ confidence intervals of the $\mathrm{IC}_{50}$ values; doxorubicin: ambiguous fitting, no confidence interval available. Significantly stronger activities comparing to that of 1 are marked; *: $p<0.05$ by unpaired T-test; ${ }^{* *}$ : $\mathrm{p}<0.001$ by ANOVA.

doi:10.1371/journal.pone.0023922.g006 chain can be beneficial on the activity comparing to that of the non-substituted protoapigenone, while this is not true in case of the naphthoflavone derivatives.

3. Based on these findings, new candidates for further studies were discovered, among which protoapigenone 1'-O-butyl ether $(\mathbf{7})$ is the most promising one. The in vivo study of this compound is planned in the near future, and the synthesis of further derivatives is currently in progress.

\section{Materials and Methods}

\section{General}

Solvents, reagents and chromatographic stationary phases were purchased from the following companies: solvents and octadecyl silica from Merck Chemicals, PIFA and Sephadex LH-20 gel from Sigma and $\beta$-naphthoflavone from Indofine Chemical Company Ltd. (NJ, USA). Apigenin was synthesized from naringenine (Indofine Ltd.) by Chuang DW in previous experiments (not discussed here). All compounds tested possessed a purity of over 95\%, except for $\mathbf{1}^{\prime \prime}(89.1 \%), 3(94.8 \%)$ and 6 (94.8\%). Compound purities were checked using HPLC-UV with one of the solvent systems described below at $245 \mathrm{~nm}$ using a gradient system consisting of two Jasco PU2080 LC pump and a Jasco UV2075 ultraviolet detector connected to a Hercule 2000 chromatographic interface. Columns used: Agilent Zorbax XDB-C8, $5 \mu \mathrm{m}$, $150 \times 4.6 \mathrm{~mm}$ (RP-HPLC), and Agilent Zorbax-sil $5 \mu \mathrm{m}, 250 \times$ $4.6 \mathrm{~mm}$ (NP-HPLC). Solvent systems: RP-HPLC ${ }_{1}$ : 55\% aqueous $\mathrm{MeOH}$ increasing to $80 \%$ in 10 minutes; RP-HPLC 2 : $35 \% \mathrm{v} / \mathrm{v}$ aqueous $\mathrm{MeOH}$ increasing to $80 \%$ in 10 minutes; NP-HPLC: $5 \%$ 
$\mathrm{v} / \mathrm{v}$ isopropanol in dichloromethane increasing to $17 \%$ in $12 \mathrm{~min}-$ utes and flow rate was $1 \mathrm{~mL} / \mathrm{min}$ in each case. Chromatograms are shown in Figures S4-S20. ${ }^{1} \mathrm{H}$ NMR spectra were taken using a Varian Gemini-2000 $200 \mathrm{MHz}$ FT-NMR, and ${ }^{13} \mathrm{C}$ and 2D NMR spectra were taken using a Varian Mercury-plus $400 \mathrm{MHz}$ FTNMR spectrometer. Shifts are given in ppm downfield of TMS and were referenced to internal residual protosolvent. ${ }^{1} \mathrm{H}$ NMR spectra of all new compounds are shown in Figures S21-S60. These spectra were taken prior to crystallization, while activity assays and HPLC were done with the pure crystals obtained for all compounds, except for $\mathbf{1}^{\prime \prime}$ which could not be crystallized. Hence, Figures S22-S60. do not represent final purity and are only shown to assist interpretation of NMR data. Mass spectra were measured on a PECIEX API 3000 instrument with a turbo ion spray source, Agilent-1100, LC/MSDTrap, or Shimadzu LCMS-IT-TOF with an ESI interface. Crystallographic data for structural analysis have been deposited with the Cambridge Crystallographic Data Centre (deposition numbers CCDC 762721-762723). These data sets can be obtained free of charge via www.ccdc.cam.ac.uk/conts/retrieving html, email: (or from the CGDC, 12 Union Road, Cambridge CB2 1EZ, UK; fax: +44 1223336 033; e-mail: deposit@ccdc.camac.uk). The melting points were measured on VEB Wagetechnik Rapido PHNK melt microscope (upper limit: $360^{\circ} \mathrm{C}$ ). The structures of $\mathbf{1}, \mathbf{1}^{\prime}, \mathbf{1 1}$ and 12 were determined based on literature data $[6,12,19]$, their melting points were found $183-184^{\circ} \mathrm{C}$ (lit. for $\mathbf{1}$ of natural origin: $180-181^{\circ} \mathrm{C}$ [6]), 328-330 (lit. for $\mathbf{1}^{\prime}$ of natural origin: $328-330^{\circ} \mathrm{C}$ [20]), 197$198^{\circ} \mathrm{C}$ and $171-183^{\circ} \mathrm{C}$, respectively. HPLC and purity data for the known compounds $\mathbf{1}, \mathbf{1 1}$ and $\mathbf{1 2}$ are as follows. 1. RP-HPLC : $_{1}$ $7.057 \mathrm{~min}$ (Figure S4), purity: 95.9\%. 11. NP-HPLC: $9.733 \mathrm{~min}$ (Figure S13), purity: 98.8\%. 12. NP-HPLC: $10.580 \mathrm{~min}$ (Figure S14), purity: $99.5 \%$. Yields given represent isolated yields.

\section{Assay for Cytotoxicity}

Human breast (MCF-7 and MDA-MB-231), liver (HepG2 and Hep3B), and lung (A549) cancer cell lines were obtained from the American Type Culture Collection. The human oral squamous cell carcinoma Ca9-22 was a kind gift from Dr. Jeff Yi-Fu Chen, Kaohsiung Medical University, Kaohsiung, Taiwan. All cell lines were propagated in RPMI-1640 medium supplemented with $10 \%$ (v/v) FBS, $100 \mathrm{U} / \mathrm{mL}$ penicillin, and $100 \mu \mathrm{g} / \mathrm{mL}$ streptomycin at $37^{\circ} \mathrm{C}$ in a humidified atmosphere of $5 \% \mathrm{CO}_{2}$ and $95 \%$ air. Cell viability was measured by the MTT colorimetric method [21]. Cells were seeded at densities of 5,000 to 10,000 cells/well in 96well tissue culture plates. On day two, cells were treated with the test compounds for another $72 \mathrm{~h}$. After drug treatment, attached cells were incubated with MTT $(0.5 \mathrm{mg} / \mathrm{mL}, 1 \mathrm{~h})$ and subsequently solubilized in DMSO. The absorbtion at $550 \mathrm{~nm}$ was then measured using a microplate reader. The $\mathrm{IC}_{50}$ was recorded as the concentration of the agent that reduced cell viability by $50 \%$ under the experimental conditions, and it was determined by applying variable slope nonlinear regression with automatic outlier elimination at $\mathrm{Q}=1.0 \%$ by using GraphPad Prism $5.0(\mathrm{GraphPad}$ Software Inc.). Statistical evaluation of the individual $\mathrm{IC}_{50}$ values was done by one way ANOVA with Dunnet's Multiple Comparison Test by choosing compounds $\mathbf{1}$ and $\mathbf{1 1}$ as references to their corresponding 1'-O-alkylated analogs. To reveal structureactivity relationships, the activites of each compound were compared by Bonferroni tests, and, in case of comparing the activities of $\mathbf{1}$ and $\mathbf{7}$, unpaired T-tests were also performed.

\section{General procedure for 1}

Compound 2 (100.0 mg) was dissolved in ACN:water (9:1, v/v; $100 \mathrm{~mL})$. A quantity of PIFA (318.0 mg, 2 eq.) was added to the container, and microwave heating was applied $\left(70^{\circ} \mathrm{C}\right.$, max. power
$500 \mathrm{~W}, 1 \mathrm{~min}$ ) with continuous stirring. The resulting mixture was evaporated, adsorbed onto $2.0 \mathrm{~g}$ of octadecyl silica, layered on the top of $2.0 \mathrm{~g}$ of octadecyl silica, washed with $10 \mathrm{~mL}$ of $20 \%$ aqueous $\mathrm{MeOH}$ and eluted with $20 \mathrm{~mL}$ of $60 \%$ aq. $\mathrm{MeOH}$. The latter fraction was evaporated, redissolved in $\mathrm{MeOH}$, and purified on a Sephadex LH-20 column $(385 \times 20 \mathrm{~mm}$; eluent: $\mathrm{MeOH})$. Fractions of $10 \mathrm{~mL}$ were collected, and fractions 11 through 14 gave protoapigenone $(33.0 \mathrm{mg}, 31.2 \%)$. The side-products $\mathbf{1}^{\prime}$ and $\mathbf{1}^{\prime \prime}$ were isolated from fractions 15 through 18 and 19 through 21, respectively, with repeated purification on the same Sephadex LH20 column.

\section{Scale-up procedure for 1}

Different quantities of $\mathbf{2}(800.0 \mathrm{mg}, 2.0 \mathrm{~g}$, and $5.0 \mathrm{~g}$ ) were dissolved in ACN:water $(9: 1, \mathrm{v} / \mathrm{v} ; 800 \mathrm{~mL}, 2 \mathrm{~L}$ and $5 \mathrm{~L})$ in staunch containers that were then pre-heated to $70^{\circ} \mathrm{C}$. After releasing the pressure, PIFA (2 eq.; $2.55 \mathrm{~g}, 6.37 \mathrm{~g}$ and $15.93 \mathrm{~g}$ ) was added to each solution in a careful manner to prevent sudden explosive boiling, and after re-sealing the containers, microwave heating was applied $\left(70^{\circ} \mathrm{C}\right.$, max. $\left.500 \mathrm{~W}, 3 \mathrm{~min}\right)$ with continuous stirring. The samples obtained were cooled under a stream of water and subsequently with an ice bath. Each sample was immediately evaporated in a $10 \mathrm{~L}$ roundbottom flask using a Büchi Rotavapor R-220 at $50^{\circ} \mathrm{C}$. After redissolving them, the samples were adsorbed onto octadecyl silica $(16.0 \mathrm{~g}, 40.0 \mathrm{~g}$ or $100.0 \mathrm{~g}$ ) that was layered on top of the same amount of octadecyl silica. SPE was performed by eluting with $15 \%$ aqueous $\mathrm{MeOH}$ (80 mL, $200 \mathrm{~mL}$ or $500 \mathrm{~mL}$ ) followed by $50 \%$ aqueous $\mathrm{MeOH}$ $(200 \mathrm{~mL}, 500 \mathrm{~mL}$ and $1200 \mathrm{~mL}$. The fractions containing $50 \%$ $\mathrm{MeOH}$ were purified on silica gel with $\mathrm{CH}_{2} \mathrm{Cl}_{2}-\mathrm{MeOH}(20: 1$, v/v) to give $251.3 \mathrm{mg}(29.6 \%), 546.2 \mathrm{mg}(25.8 \%)$ and $1.18 \mathrm{~g}(22.3 \%)$ of protoapigenone, respectively.

\section{General procedure for 3 through 9 and 11 through 18}

A total of $50.0 \mathrm{mg}$ of $\mathbf{2}$ or $\mathbf{1 0}$ was dissolved in $50 \mathrm{~mL}$ of a 9:1 v/ $\mathrm{v}$ mixture of acetonitrile and the corresponding alcohol $(\mathrm{MeOH}$, $\mathrm{EtOH}, \mathrm{PrOH}, \mathrm{PrOH}, \mathrm{BuOH}$, allyl alcohol or propargyl alcohol) or water in the case of $\mathbf{1 1}$ and reacted with PIFA (159 mg or $150 \mathrm{mg}, 2$ eq.) under the same conditions used to obtain $\mathbf{1}$. To purify the products, flash chromatography (Biotage Flash+) on silica columns (Biotage Si 24+M 2758-1; $11 \times 150 \mathrm{~mm}$ ) was performed using a dry-loading technique and $n$-hexane:EtOAc:acetone solvent systems (all ratios are given in v/v/v; 7:3:1 for 3-5, 9:3:1 for $\mathbf{6 , 8}$ and $\mathbf{9}$, and 11:3:1 for $\mathbf{7}$ each containing $0.01 \%$ TFA, as well as 16:3:1 for $\mathbf{1 1}$ and 12, 27:3:1 for 13, 36:3:1 for 14, 15, 17 and $\mathbf{1 8}$, and 44:3:1 for $\mathbf{1 6}$ (no added acid was used)) at flow rates of $24 \mathrm{~mL} / \mathrm{min}$. Finally, $\mathbf{3}$ through $\mathbf{9}$ and $\mathbf{1 2}$ were crystallized from hexane:acetone $(1: 1, \mathrm{v} / \mathrm{v})$, while $\mathbf{1 3}$ through $\mathbf{1 8}$ were crystallized from hexane: $\mathrm{CH}_{2} \mathrm{Cl}_{2}(1: 1, \mathrm{v} / \mathrm{v})$.

\section{Experimental}

Protoapigenone-(8-3")-apigenin (1"). Yield: 4\% (8.2 mg) as a side-product of the reaction of $\mathbf{2}$ into $\mathbf{1}$; RP-HPLC $\mathrm{R}_{2}: 6.843 \mathrm{~min}$ (Figure S5), purity: 89.1\%; yellow solid, mp: $>360^{\circ} \mathrm{C},{ }^{1} \mathrm{H}$ NMR (acetone- $\left.\mathrm{d}_{6}\right) \delta 12.87\left(1 \mathrm{H}, \mathrm{s}, \mathrm{OH}-5^{\prime \prime}\right), 12.65(1 \mathrm{H}, \mathrm{s}, \mathrm{OH}-5), 7.38$ $\left(2 \mathrm{H}, \mathrm{d}, \mathcal{f}=8.8 \mathrm{~Hz}, \mathrm{H}-2^{\prime \prime \prime}\right.$ and $\left.\mathrm{H}-3^{\prime \prime \prime}\right), 6.81$ ( $1 \mathrm{H}, \mathrm{dd}, \mathcal{f}=10.0$, $3.2 \mathrm{~Hz}, \mathrm{H}-2^{\prime}$ or $\left.\mathrm{H}-6^{\prime}\right), 6.78\left(2 \mathrm{H}, \mathrm{d}, \mathcal{F}=8.8 \mathrm{~Hz}, \mathrm{H}-3^{\prime \prime \prime}\right.$ and $\left.\mathrm{H}-5^{\prime \prime \prime}\right)$, $6.76\left(1 \mathrm{H}, \mathrm{dd}, \mathcal{f}=9.6,3.2 \mathrm{~Hz}, \mathrm{H}-6^{\prime}\right.$ or $\left.\mathrm{H}-2^{\prime}\right), 6.57(1 \mathrm{H}, \mathrm{s}, \mathrm{H}-3)$, $6.50\left(1 \mathrm{H}, \mathrm{d}, \mathcal{f}=2.0 \mathrm{~Hz}, \mathrm{H}-8^{\prime \prime}\right), 6.30(1 \mathrm{H}, \mathrm{s}, \mathrm{H}-6), 6.29(1 \mathrm{H}, \mathrm{d}$, $\left.\mathcal{f}=2.0 \mathrm{~Hz}, \mathrm{H}-6^{\prime \prime}\right), 6.08\left(1 \mathrm{H}, \mathrm{dd}, \mathcal{F}=10.0,2.0 \mathrm{~Hz}, \mathrm{H}-3^{\prime}\right.$ or $\left.\mathrm{H}-5^{\prime}\right)$, $5.85\left(1 \mathrm{H}, \mathrm{dd}, \mathcal{f}=10.0,1.6 \mathrm{~Hz}, \mathrm{H}-5^{\prime}\right.$ or $\left.\mathrm{H}-3^{\prime}\right)$ (Figures $\mathrm{S} 21$ and $\mathrm{S} 22) ;{ }^{13} \mathrm{C}$ NMR (acetone-d 6 ) $\delta 185.2\left(\mathrm{C}-4^{\prime}\right), 184.1(\mathrm{C}-4), 182.5(\mathrm{C}-$ $\left.4^{\prime \prime}\right), 168.8(\mathrm{C}-2), 165.8\left(\mathrm{C}-7^{\prime \prime}\right), 165.4\left(\mathrm{C}-2^{\prime \prime}\right), 164.0(\mathrm{C}-8 \mathrm{a}), 163.9(\mathrm{C}-$ 7, C-5"), $163.5(\mathrm{C}-5), 161.4\left(\mathrm{C}-4^{\prime \prime \prime}\right), 159.5\left(\mathrm{C}-8 \mathrm{a}^{\prime \prime}\right), 148.1\left(\mathrm{C}-2^{\prime}\right.$ or 
C-6'), 148.0 (C-6' or C-2'), $131.6\left(\mathrm{C}-2^{\prime \prime \prime}, \mathrm{C}-6^{\prime \prime \prime}\right), 130.6$ (C-3' or C$\left.5^{\prime}\right), 130.5\left(\mathrm{C}-5^{\prime}\right.$ or $\left.\mathrm{C}-3^{\prime}\right), 125.3$ (C-1"'), 116.7 (C-3"', C-5"'), 111.3 (C-3"), 108.0 (C-3), 106.1 (C-4a), 105.3 (C-4a"), 101.3 (C-8), 100.5 (C-6), $100.3\left(\mathrm{C}^{-6} \mathrm{6}^{\prime \prime}\right), 95.4\left(\mathrm{C}-8^{\prime \prime}\right), 71.3\left(\mathrm{C}^{\prime} \mathrm{l}^{\prime}\right)$; ESI-MS $(\mathrm{m} / z, \%): 555$ $\left(\mathrm{M}^{+}+\mathrm{H}, \quad 100\right), \quad 577 \quad\left(\mathrm{M}^{+}+\mathrm{Na}, \quad 92\right) ; \quad$ HRMS: $\mathrm{C}_{30} \mathrm{H}_{18} \mathrm{O}_{11} \mathrm{Na}$, calculated 577.0747; found 577.0750.

Protoapigenone $\mathbf{1}^{\prime}$-O $\boldsymbol{O}$-methylether (3). Yield: $42 \%(23.3$ $\mathrm{mg}$ ); RP-HPLG ${ }_{1}$ : 3.860 min (Figure S6), purity: 94.8\%; white crystals, mp: $153-155^{\circ} \mathrm{C},{ }^{1} \mathrm{H}$ NMR (acetone-d $\left.{ }_{6}\right) \delta 12.59(1 \mathrm{H}, \mathrm{br}$, $\mathrm{OH}-5), 6.99\left(2 \mathrm{H}, \mathrm{d}, \mathcal{f}=10.2 \mathrm{~Hz}, \mathrm{H}-2^{\prime}\right.$ and $\left.\mathrm{H}-6^{\prime}\right), 6.55(2 \mathrm{H}, \mathrm{d}$, $7=10.2 \mathrm{~Hz}, \mathrm{H}-3^{\prime}$ and $\left.\mathrm{H}-5^{\prime}\right), 6.50(1 \mathrm{H}, \mathrm{s}, \mathrm{H}-3), 6.29(1 \mathrm{H}, \mathrm{d}$, $f=2.0 \mathrm{~Hz}, \mathrm{H}-8), 6.24(1 \mathrm{H}, \mathrm{d}, \mathcal{f}=2.0 \mathrm{~Hz}, \mathrm{H}-6), 3.42(3 \mathrm{H}, \mathrm{s}$, $\mathrm{OCH}_{3}-\mathrm{1}^{\prime}$ ) (Figures S23 and S24); ${ }^{13} \mathrm{C}$ NMR (acetone-d 6 ) $\delta 183.4$ (C-4'), 181.6 (C-4), 164.7 (C-2), 164.0 (C-7), 162.0 (C-5), 157.5 (G8a), 144.8 (C-2' and C-6'), 132.4 (C-3' and C-5'), 106.9 (C-3), 104.3 (C-4a), 98.8 (C-6), 93.5 (C-8), 74.2 (C-1'), 51.6 (C-1"); ESIMS $(\mathrm{m} / z, \%): 323\left(\mathbf{M}^{+}+\mathrm{Na}, 100\right), 301\left(\mathbf{M}^{+}+\mathrm{H}, 82\right)$; HRMS: $\mathrm{C}_{16} \mathrm{H}_{12} \mathrm{O}_{6} \mathrm{Na}$, calculated 323.0532; found 323.0530.

Protoapigenone 1'-O-ethylether (4). Yield: 33\% (19.2 mg); RP-HPLG $_{1}$ : 5.157 min (Figure S7), purity: 96.7\%; white crystals, mp: $170-172^{\circ} \mathrm{C},{ }^{1} \mathrm{H}$ NMR (acetone-d $\left.{ }_{6}\right) \delta 12.59$ (1H, br, OH-5), $7.01\left(2 \mathrm{H}, \mathrm{d}, \mathcal{f}=10.2 \mathrm{~Hz}, \mathrm{H}-2^{\prime}\right.$ and H-6'), $6.56(1 \mathrm{H}, \mathrm{s}, \mathrm{H}-3), 6.51$ $\left(2 \mathrm{H}, \mathrm{d}, \mathcal{f}=10.0 \mathrm{~Hz}, \mathrm{H}-3^{\prime}\right.$ and $\left.\mathrm{H}-5^{\prime}\right), 6.28(1 \mathrm{H}, \mathrm{br}, \mathrm{H}-8), 6.24(1 \mathrm{H}$, br, H-6), 3.63 (2H, q, $\left.7=3.5 \mathrm{~Hz}, \mathrm{H}-\mathrm{l}^{\prime \prime}\right), 1.27$ (3H, s, H-2") (Figures S25, S26 and S27); ${ }^{13} \mathrm{C}$ NMR (acetone-d 6 ) $\delta 183.6\left(\mathrm{C}-4^{\prime}\right), 181.7$ (C-4), 164.9 (C-2), 163.9 (C-7), 162.0 (C-5), 157.5 (C-8a), 145.3 (C$2^{\prime}$ and $\left.\mathrm{C}-6^{\prime}\right), 131.9\left(\mathrm{C}-3^{\prime}\right.$ and $\left.\mathrm{C}-5^{\prime}\right), 106.9(\mathrm{C}-3), 104.3(\mathrm{C}-4 \mathrm{a})$, $98.7(\mathrm{C}-6), 93.4(\mathrm{C}-8), 74.0\left(\mathrm{C}^{-1}{ }^{\prime}\right), 60.0\left(\mathrm{C}^{\prime} \mathbf{1}^{\prime \prime}\right), 14.6\left(\mathrm{C}-2^{\prime \prime}\right)$; ESIMS $(\mathrm{m} / \mathrm{z}, \%): 337\left(\mathbf{M}^{+}+\mathrm{Na}, 100\right), 315\left(\mathbf{M}^{+}+\mathrm{H}, 96\right)$; HRMS $\mathrm{C}_{17} \mathrm{H}_{14} \mathrm{O}_{6} \mathrm{Na}$, calculated 337.0688; found 337.0686.

Protoapigenone $\quad \mathbf{1}^{\prime}$-O-propylether (5). Yield: $36 \%$ (21.9 mg); RP-HPLC 1 : $8.240 \mathrm{~min}$ (Figure S8), purity: 97.8\%; white crystals, mp: $181-182^{\circ} \mathrm{C},{ }^{1} \mathrm{H}$ NMR (acetone- $\mathrm{d}_{6}$ ) $\delta 12.59$ $(1 \mathrm{H}, \mathrm{br}, \mathrm{OH}-5), 7.01\left(2 \mathrm{H}, \mathrm{d}, \mathcal{F}=10.2 \mathrm{~Hz}, \mathrm{H}-2^{\prime}\right.$ and $\left.\mathrm{H}-6^{\prime}\right), 6.56(1 \mathrm{H}$, s, H-3), $6.52\left(2 \mathrm{H}, \mathrm{d}, f=10.2 \mathrm{~Hz}, \mathrm{H}-3^{\prime}\right.$ and $\left.\mathrm{H}-5^{\prime}\right), 6.28(1 \mathrm{H}, \mathrm{d}$, $\mathcal{f}=2 \mathrm{~Hz}, \mathrm{H}-8), 6.24(1 \mathrm{H}, \mathrm{d}, \mathcal{f}=2 \mathrm{~Hz}, \mathrm{H}-6), 3.54(2 \mathrm{H}, \mathrm{t}, \mathcal{f}=6.3 \mathrm{~Hz}$, H-1"), $1.68\left(2 \mathrm{H}, \mathrm{m}, \mathrm{H}-2^{\prime \prime}\right), 0.98\left(3 \mathrm{H}, \mathrm{t}, \mathcal{F}=7.3 \mathrm{~Hz}, \mathrm{H}-3^{\prime \prime}\right)$ (Figures S28, S29 and S30); ${ }^{13} \mathrm{C}$ NMR (acetone- $\left.\mathrm{d}_{6}\right) \delta 183.6\left(\mathrm{C}-4^{\prime}\right), 181.7(\mathrm{C}-$ 4), 164.8 (C-2), 163.9 (C-7), 162.0 (C-5), 157.5 (C-8a), 145.4 (C-2' and C-6' $), 131.9\left(\mathrm{C}^{\prime}-3^{\prime}\right.$ and $\left.\mathrm{C}^{-} 5^{\prime}\right), 106.9(\mathrm{C}-3), 104.3(\mathrm{C}-4 \mathrm{a}), 98.7(\mathrm{C}-$ 6), $93.5(\mathrm{C}-8), 74.0\left(\mathrm{C}-1^{\prime}\right), 65.9\left(\mathrm{C}-1^{\prime \prime}\right), 22.7\left(\mathrm{C}-2^{\prime \prime}\right), 9.6\left(\mathrm{C}-3^{\prime \prime}\right)$; ESIMS $(m / z, \%): 329\left(\mathbf{M}^{+}+\mathrm{H}, 100\right), 351\left(\mathbf{M}^{+}+\mathrm{Na}, 80\right)$; HRMS $\mathrm{C}_{18} \mathrm{H}_{16} \mathrm{O}_{6} \mathrm{Na}$, calculated 351.0845; found 351.0846.

Protoapigenone $\quad \mathbf{1}^{\prime}$-O-isopropylether (6). Yield: $26 \%$ (15.8 mg); RP-HPLC 1 : $7.637 \mathrm{~min}$ (Figure S9), purity: 94.9\%; light-brownish crystals, mp: $138-140^{\circ} \mathrm{C},{ }^{1} \mathrm{H}$ NMR (acetone- $\left.\mathrm{d}_{6}\right) \delta$ $12.59(1 \mathrm{H}, \mathrm{br}, \mathrm{OH}-5), 7.07\left(2 \mathrm{H}, \mathrm{d}, \mathcal{f}=9.8 \mathrm{~Hz}, \mathrm{H}-2^{\prime}\right.$ and $\left.\mathrm{H}-6^{\prime}\right)$, $6.56(1 \mathrm{H}, \mathrm{s}, \mathrm{H}-3), 6.50\left(2 \mathrm{H}, \mathrm{d}, \mathcal{J}=9.8 \mathrm{~Hz}, \mathrm{H}-3^{\prime}\right.$ and $\left.\mathrm{H}-5^{\prime}\right), 6.28$ (1H, br, H-8), 6.24 (1H, br, H-6), $3.94\left(1 \mathrm{H}, \mathrm{m}, \mathcal{f}=6.1 \mathrm{~Hz}, \mathrm{H}-1^{\prime \prime}\right)$, $1.24\left(6 \mathrm{H}, \mathrm{d}, \mathcal{f}=6.2 \mathrm{~Hz}, \mathrm{H}-2^{\prime \prime}\right.$ and $\mathrm{H}-3^{\prime \prime}$ ) (Figures S31, S32, S33 and S34); ${ }^{13} \mathrm{C}$ NMR (acetone- $\left.\mathrm{d}_{6}\right) \delta 162.0(\mathrm{C}-5), 145.7\left(\mathrm{C}-2^{\prime}\right.$ and C-6'), $131.3\left(\mathrm{C}^{\prime}-3^{\prime}\right.$ and C-5'), 107.0 (C-3), 104.2 (C-4a), 98.7 (C-6), $93.4(\mathrm{C}-8), 68.0\left(\mathrm{C}-1^{\prime \prime}\right), 23.5\left(\mathrm{C}-2^{\prime \prime}\right.$ and C-3"); ESI-MS $(\mathrm{m} / z, \%)$ : $329\left(\mathrm{M}^{+}+\mathrm{H}, 100\right), 351\left(\mathrm{M}^{+}+\mathrm{Na}, 84\right), 270\left(\mathrm{M}^{+}+\mathrm{H}-\mathrm{OC}_{3} \mathrm{H}_{6}, 16\right)$; HRMS $\mathrm{C}_{18} \mathrm{H}_{16} \mathrm{O}_{6} \mathrm{Na}$, calculated 351.0845; found 351.0844.

Protoapigenone 1' -O-butylether (7). Yield: 44\% (27.9 mg); RP-HPLC $_{1}$ : $9.437 \mathrm{~min}$ (Figure S10), purity: $96.1 \%$; white crystals, mp: $167-168^{\circ} \mathrm{C},{ }^{1} \mathrm{H}$ NMR (acetone- $\left.\mathrm{d}_{6}\right) \delta 12.60(1 \mathrm{H}, \mathrm{br}, \mathrm{OH}-5)$, $7.01\left(2 \mathrm{H}, \mathrm{d}, \mathcal{J}=10.8 \mathrm{~Hz}, \mathrm{H}-2^{\prime}\right.$ and H-6 $\left.{ }^{\prime}\right), 6.55$ (1H, s, H-3), 6.52 $\left(2 \mathrm{H}, \mathrm{d}, \mathcal{f}=10.8 \mathrm{~Hz}, \mathrm{H}-3^{\prime}\right.$ and $\left.\mathrm{H}-5^{\prime}\right), 6.28(1 \mathrm{H}, \mathrm{br}, \mathrm{H}-8), 6.25(1 \mathrm{H}$, br, H-6), 3.58 (2H, t, $\left.\mathcal{f}=6.0 \mathrm{~Hz}, \mathrm{H}-\mathrm{1}^{\prime \prime}\right), 1.63\left(2 \mathrm{H}, \mathrm{m}, \mathrm{br}, \mathrm{H}-2^{\prime \prime}\right), 1.44$ $\left(2 \mathrm{H}, \mathrm{m}, \mathrm{br}, \mathrm{H}-3^{\prime \prime}\right), 0.93\left(3 \mathrm{H}, \mathrm{t}, \mathcal{J}=7.2 \mathrm{~Hz}, \mathrm{H}-4^{\prime \prime}\right.$ ) (Figures S35, S36 and S37); ${ }^{13} \mathrm{C}$ NMR (acetone- $\left.\mathrm{d}_{6}\right) \delta 183.6\left(\mathrm{C}-4^{\prime}\right), 181.7(\mathrm{C}-4), 164.9$ (C-2), 163.9 (C-7), 162.0 (C-5), 157.5 (C-8a), $145.4\left(\mathrm{C}-2^{\prime}\right.$ and C-6'),
$132.0\left(\mathrm{C}^{-} 3^{\prime}\right.$ and $\left.\mathrm{C}^{-5}{ }^{\prime}\right), 106.9(\mathrm{C}-3), 104.3(\mathrm{C}-4 \mathrm{a}), 98.7(\mathrm{C}-6), 93.4(\mathrm{C}-$ 8), $74.0\left(\mathrm{C}-1^{\prime}\right), 64.1\left(\mathrm{C}-1^{\prime \prime}\right), 31.5\left(\mathrm{C}-2^{\prime \prime}\right), 18.6\left(\mathrm{C}-3^{\prime \prime}\right), 12.8\left(\mathrm{C}-4^{\prime \prime}\right)$; HRMS $\mathrm{C}_{19} \mathrm{H}_{18} \mathrm{O}_{6} \mathrm{Na}$, calculated 365.1001; found 365.1000.

Protoapigenone $\mathbf{1}^{\prime}$ - $\boldsymbol{O}$-allylether (8). Yield: $29 \%(17.5 \mathrm{mg})$; $\mathrm{RP}_{-H P L G_{1}}$ : $8.283 \mathrm{~min}$ (Figure S11), purity: 97.2\%; transparent crystals, ${ }^{1} \mathrm{H}$ NMR (acetone-d $\left.\mathrm{d}_{6}\right) \delta 12.59(1 \mathrm{H}, \mathrm{br}, \mathrm{OH}-5), 7.04(2 \mathrm{H}$, d, $\mathcal{f}=10.2 \mathrm{~Hz}, \mathrm{H}-2^{\prime}$ and H-6'), $6.57(1 \mathrm{H}, \mathrm{s}, \mathrm{H}-3), 6.53(2 \mathrm{H}, \mathrm{d}$, $\mathcal{f}=10.4 \mathrm{~Hz}, \mathrm{H}-3^{\prime}$ and $\left.\mathrm{H}-5^{\prime}\right), 6.29(1 \mathrm{H}, \mathrm{d}, \mathcal{f}=2.2 \mathrm{~Hz}, \mathrm{H}-8), 6.24$ $(1 \mathrm{H}, \mathcal{f}=2.2 \mathrm{~Hz}, \mathrm{H}-6), 6.00\left(1 \mathrm{H}, \mathrm{m}, \mathcal{f}=5.4 \mathrm{~Hz}, \mathrm{H}-2^{\prime \prime}\right), 5.37(1 \mathrm{H}, \mathrm{d}$, $\mathcal{f}=17.2 \mathrm{~Hz}, \mathrm{H}-3^{\prime \prime}$, cis), $5.20\left(1 \mathrm{H}, \mathrm{d}, \mathcal{J}=10.4 \mathrm{~Hz}, \mathrm{H}-3^{\prime \prime}\right.$, trans), 4.14 $\left(2 \mathrm{H}, \mathcal{f}=5.2 \mathrm{~Hz}, \mathrm{H}-1^{\prime \prime}\right)$ (Figures S38 and S39); ${ }^{13} \mathrm{C}$ NMR (acetone$\left.\mathrm{d}_{6}\right) \delta 164.9(\mathrm{C}-2), 163.9(\mathrm{C}-7), 162.0(\mathrm{C}-5), 157.5(\mathrm{C}-8 \mathrm{a}), 145.4(\mathrm{C}-$ $2^{\prime}$ and C-6'), $132.0\left(\mathrm{C}^{\prime}-3^{\prime}\right.$ and C-5'), $106.9(\mathrm{C}-3), 98.7(\mathrm{C}-6), 93.4$ (C-8), $72.9\left(\mathrm{C}^{-1}{ }^{\prime}\right), 65.9\left(\mathrm{C}_{-1} \mathrm{1}^{\prime \prime}\right)$; ESI-MS $(\mathrm{m} / \mathrm{z}, \%): 349\left(\mathrm{M}^{+}+\mathrm{Na}\right.$, 100), $327\left(\mathrm{M}^{+}+\mathrm{H}\right.$, 85); HRMS $\mathrm{C}_{18} \mathrm{H}_{14} \mathrm{O}_{6} \mathrm{Na}$, calculated 349.0688; found 349.0690; X-ray data are shown in Figure S1.

Protoapigenone $\mathbf{1}^{\prime}$-O-propargylether (9). Yield: $36 \%$ (21.6 mg); RP-HPLC 1 : 4.180 min (Figure S12), purity: 97.4\%; yellow crystalline powder, mp: $205-207^{\circ} \mathrm{C},{ }^{1} \mathrm{H}$ NMR (acetone- $\mathrm{d}_{6}$ ) $\delta 12.55(1 \mathrm{H}, \mathrm{s}, \mathrm{OH}-5), 7.08\left(2 \mathrm{H}, \mathrm{d}, \mathcal{F}=10.0 \mathrm{~Hz}, \mathrm{H}-2^{\prime}\right.$ and $\left.\mathrm{H}-6^{\prime}\right)$, $6.55\left(2 \mathrm{H}, \mathrm{d}, \mathcal{f}=10.2 \mathrm{~Hz}, \mathrm{H}-3^{\prime}\right.$ and $\left.\mathrm{H}-5^{\prime}\right), 6.53(1 \mathrm{H}, \mathrm{s}, \mathrm{H}-3), 6.30$ $(1 \mathrm{H}, \mathrm{d}, \mathcal{f}=2.2 \mathrm{~Hz}, \mathrm{H}-8), 6.24(1 \mathrm{H}, \mathrm{d}, \mathcal{F}=2.0 \mathrm{~Hz}, \mathrm{H}-6), 4.34(2 \mathrm{H}$, $\left.\mathrm{d}, \mathcal{f}=2.4 \mathrm{~Hz}, \mathrm{H}-\mathrm{l}^{\prime \prime}\right), 3.11\left(1 \mathrm{H}, \mathrm{t}, \mathcal{f}=2.4 \mathrm{~Hz}, \mathrm{H}-3^{\prime \prime}\right)$ (Figures $\mathrm{S} 40$ and $\mathrm{S} 41,400 \mathrm{MHz}{ }^{1} \mathrm{H}$ spectrum is also provided in Figure $\mathrm{S} 42$ ); ${ }^{13} \mathrm{C}$ NMR (acetone-d $\left.\mathrm{d}_{6}\right) \delta 185.4\left(\mathrm{C}-4^{\prime}\right), 183.5(\mathrm{C}-4), 166.1(\mathrm{C}-2)$, 166.0 (C-7), 163.9 (C-5), 159.4 (C-8a), 145.9 (C-2' and C-6'), $134.3\left(\mathrm{C}^{-} 3^{\prime}\right.$ and $\left.\mathrm{C}^{-5}{ }^{\prime}\right), 109.0(\mathrm{C}-3), 106.2(\mathrm{C}-4 \mathrm{a}), 100.8(\mathrm{C}-6), 95.4$ (C-8), 81.3 (C-1'), 78.0 (C-3"), 76.4 (C-2"), 54.8 (C-1"); HRMS $\mathrm{C}_{18} \mathrm{H}_{12} \mathrm{O}_{6} \mathrm{Na}$, calculated 347.0532; found 347.0535.

3-(1-Ethoxy-4-oxocyclohexa-2,5-dienyl)- $1 H$-benzo $[\boldsymbol{f}]$ chromen-1-one (13). Yield: 65\% (37.5 mg); NP-HPLC: 7.507 min (Figure S15), purity: $99.8 \%$; white crystals, mp: $158-160^{\circ} \mathrm{C}$, ${ }^{1} \mathrm{H}$ NMR (CDCl3) $\delta 9.97(1 \mathrm{H}, \mathrm{d}, \mathcal{F}=8.4 \mathrm{~Hz}, \mathrm{H}-5), 8.04(1 \mathrm{H}, \mathrm{d}$, $\mathcal{f}=8.8 \mathrm{~Hz}, \mathrm{H}-9), 7.87(1 \mathrm{H}, \mathrm{dd}, \mathcal{f}=7.6,1.0 \mathrm{~Hz}, \mathrm{H}-8), 7.74(1 \mathrm{H}, \mathrm{td}$, $\mathcal{f}=8.4,1.4 \mathrm{~Hz}, \mathrm{H}-7), 7.60(1 \mathrm{H}, \mathrm{td}, \mathcal{f}=8.0,1.0 \mathrm{~Hz}, \mathrm{H}-6), 7.36$ $(1 \mathrm{H}, \mathrm{d}, \quad \mathcal{f}=9.2 \mathrm{~Hz}, \mathrm{H}-10), 6.95(1 \mathrm{H}, \mathrm{s}, \mathrm{H}-3), 6.89(2 \mathrm{H}, \mathrm{d}$, $\mathcal{f}=10.2 \mathrm{~Hz}, \mathrm{H}-2^{\prime}$ and $\left.\mathrm{H}-6^{\prime}\right), 6.55\left(2 \mathrm{H}, \mathrm{d}, \mathcal{F}=10.4 \mathrm{~Hz}, \mathrm{H}-3^{\prime}\right.$ and H-5'), 3.61 (2H, q, $\left.\mathcal{f}=7.0 \mathrm{~Hz}, \mathrm{H}-\mathrm{l}^{\prime \prime}\right), 1.307(3 \mathrm{H}, \mathrm{t}, \mathcal{f}=7.0 \mathrm{~Hz}, \mathrm{H}-$ $2^{\prime \prime}$ ) (Figures S43, S44 and S45); ${ }^{13} \mathrm{C}$ NMR (CDCl3) $\delta 184.2\left(\mathrm{C}-4^{\prime}\right)$, 179.5 (C-4), $160.9(\mathrm{C}-2), 156.9(\mathrm{C}-10 \mathrm{a}), 145.8\left(\mathrm{C}^{\prime} 2^{\prime}\right.$ and $\left.\mathrm{C}-6^{\prime}\right)$, $132.2\left(\mathrm{C}^{-} 3^{\prime}\right.$ and $\left.\mathrm{C}-5^{\prime}\right), 135.3,130.2,129.9,129.0,127.8,126.6$, 126.4 (C-4b, C-5, C-6, C-7, C-8, C-8a, C-9), 116.9 (C-10), 116.9 $(\mathrm{C}-4 \mathrm{a}), 112.1(\mathrm{C}-3), 73.9\left(\mathrm{C}^{-1}{ }^{\prime}\right), 60.5\left(\mathrm{C}-1^{\prime \prime}\right), 15,3\left(\mathrm{C}-2^{\prime \prime}\right)$; ESI-MS $(\mathrm{m} / \mathrm{z}, \quad \%): 355 \quad\left(\mathbf{M}^{+}+\mathrm{Na}, \quad 100\right), 333 \quad\left(\mathbf{M}^{+}+\mathrm{H}, \quad 79\right)$; HRMS $\mathrm{C}_{21} \mathrm{H}_{16} \mathrm{O}_{4} \mathrm{Na}$, calculated 355.0946; found 355.0945.

3-(1-Propoxy-4-oxocyclohexa-2,5-dienyl)-1 $H$-benzo [ $f]$ chromen-1-one (14). Yield: 64\% (38.4 mg); NP-HPLC: 5.977 min (Figure S16), purity: 98.9\%; transparent crystals, ${ }^{1} \mathrm{H}$ NMR (CDCl3) $\delta 9.96(1 \mathrm{H}, \mathrm{d}, \mathcal{F}=8.8 \mathrm{~Hz}, \mathrm{H}-5), 8.01(1 \mathrm{H}, \mathrm{d}, \mathcal{f}=9.0 \mathrm{~Hz}, \mathrm{H}-$ 9), $7.85(1 \mathrm{H}, \mathrm{d}, \mathrm{br}, \mathcal{f}=8.0 \mathrm{~Hz}, \mathrm{H}-8), 7.73(1 \mathrm{H}, \mathrm{td}, \mathcal{f}=7.0,1.6 \mathrm{~Hz}, \mathrm{H}-$ 7), $7.58(1 \mathrm{H}, \mathrm{td}, \mathcal{J}=8.0,1.2 \mathrm{~Hz}, \mathrm{H}-6), 7.33(1 \mathrm{H}, \mathrm{d}, \mathcal{f}=9.2 \mathrm{~Hz}, \mathrm{H}-$ 10), $6.92(1 \mathrm{H}, \mathrm{s}, \mathrm{H}-3), 6.87\left(2 \mathrm{H}, \mathrm{d}, \mathcal{f}=9.8 \mathrm{~Hz}, \mathrm{H}-2^{\prime}\right.$ and $\left.\mathrm{H}-6^{\prime}\right), 6.55$ $\left(2 \mathrm{H}, \mathrm{d}, \mathcal{f}=10.2 \mathrm{~Hz}, \mathrm{H}-3^{\prime}\right.$ and $\left.\mathrm{H}-5^{\prime}\right), 3.50\left(2 \mathrm{H}, \mathrm{t}, \mathcal{f}=6.2 \mathrm{~Hz}, \mathrm{H}-\mathrm{l}^{\prime \prime}\right)$, $1.68\left(2 \mathrm{H}\right.$, sextet, $\left.\mathcal{f}=7.0 \mathrm{~Hz}, \mathrm{H}-2^{\prime \prime}\right), 0.99\left(3 \mathrm{H}, \mathrm{t}, \mathcal{f}=7.3 \mathrm{~Hz}, \mathrm{H}-3^{\prime \prime}\right)$ (Figures S46, S47 and S48); ${ }^{13} \mathrm{C}$ NMR (CDCl3) $\delta 145.8\left(\mathrm{C}-2^{\prime}\right.$ and $\mathrm{C}-$ $\left.6^{\prime}\right), 132.2\left(\mathrm{C}^{\prime}-3^{\prime}\right.$ and $\left.\mathrm{C}-5^{\prime}\right), 135.3$, 129.0, 127.8, 126.6, $126.4(\mathrm{C}-4 \mathrm{~b}$, C-7, C-8, C-8a, C-9), 116.9 (C-10), 116.9 (C-4a), 112.1 (C-3), 73.9 $\left(\mathrm{C}^{-1}{ }^{\prime}\right), 66.5\left(\mathrm{C}-1^{\prime \prime}\right), 23.0\left(\mathrm{C}-2^{\prime \prime}\right), 10.2\left(\mathrm{C}-3^{\prime \prime}\right)$; ESI-MS (m/z, \%): 369 $\left(\mathrm{M}^{+}+\mathrm{Na}, 100\right), 347\left(\mathrm{M}^{+}+\mathrm{H}, 60\right)$; HRMS $\mathrm{C}_{22} \mathrm{H}_{18} \mathrm{O}_{4} \mathrm{Na}$, calculated 369.1103; found 369.1105; X-ray data are shown in Figure S2.

3-(1-Isopropoxy-4-oxocyclohexa-2,5-dienyl)- $1 H$-benzo [ $f]$ chromen-1-one (15). Yield: $52 \% \quad(31.2 \mathrm{mg})$; NP-HPLC: 6.307 min (Figure S17), purity: 99.0\%; transparent crystals, ${ }^{1} \mathrm{H}$ NMR $\left(\mathrm{CDCl}_{3}\right) \delta 9.97(1 \mathrm{H}, \mathrm{d}, \mathcal{f}=8.8 \mathrm{~Hz}, \mathrm{H}-5), 8.03(1 \mathrm{H}, \mathrm{d}$, 
$\mathcal{f}=9.0 \mathrm{~Hz}, \mathrm{H}-9), 7.94(1 \mathrm{H}, \mathrm{d}, \mathrm{br}, \mathcal{f}=8.2 \mathrm{~Hz}, \mathrm{H}-8), 7.74(1 \mathrm{H}, \mathrm{td}$, $\mathcal{f}=7.8,1.0 \mathrm{~Hz}, \mathrm{H}-7), 7.60(1 \mathrm{H}, \mathrm{td}, \mathcal{f}=7.6,1.0 \mathrm{~Hz}, \mathrm{H}-6), 7.35$ $(1 \mathrm{H}, \mathrm{d}, f=9.2 \mathrm{~Hz}, \mathrm{H}-10), 6.94(1 \mathrm{H}, \mathrm{s}, \mathrm{H}-3), 6.91(2 \mathrm{H}, \mathrm{d}$, $\mathcal{f}=10.6 \mathrm{~Hz}, \mathrm{H}-2^{\prime}$ and $\left.\mathrm{H}-6^{\prime}\right), 6.53\left(2 \mathrm{H}, \mathrm{d}, \mathcal{f}=9.8 \mathrm{~Hz}, \mathrm{H}-3^{\prime}\right.$ and

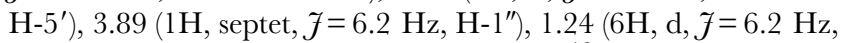
H-2" and H-3") (Figures S49, S50 and S51); ${ }^{13} \mathrm{C}$ NMR (CDCl3) $\delta$ $184.5\left(\mathrm{C}^{\prime} 4^{\prime}\right), 179.6$ (C-4), 161.2 (C-2), 156.9 (C-10a), 146.2 (C-2' and $\left.\mathrm{C}^{-} 6^{\prime}\right), 131.5\left(\mathrm{C}^{\prime}-3^{\prime}, \mathrm{C}-5^{\prime}\right), 135.3,130.2,129.9,129.0,127.7$, 126.6, 126.4 (C-4b, C-5, C-6, C-7, C-8, C-8a, C-9), 116.9 (C-10), $116.9(\mathrm{C}-4 \mathrm{a}), 112.2(\mathrm{C}-3), 74.1\left(\mathrm{C}-1^{\prime}\right), 68.5\left(\mathrm{C}-1^{\prime \prime}\right), 24.3\left(\mathrm{C}-2^{\prime \prime}\right.$ and C-3"); ESI-MS (m/z, \%): $369\left(\mathbf{M}^{+}+\mathrm{Na}, 100\right), 347\left(\mathbf{M}^{+}+\mathrm{H}, 54\right)$; HRMS $\mathrm{C}_{22} \mathrm{H}_{18} \mathrm{O}_{4} \mathrm{Na}$, calculated 369.1103; found 369.1104.

3-(1-Butoxy-4-oxocyclohexa-2,5-dienyl)- $1 H$-benzo $[f]$ chromen-1-one (16). Yield: $54 \%$ (33.8 mg); NP-HPLC: 5.230 min (Figure S18), purity: 95.8\%; transparent crystals, mp: $72-73^{\circ} \mathrm{C},{ }^{1} \mathrm{H}$ NMR $\left(\mathrm{CDCl}_{3}\right) \delta 9.97(1 \mathrm{H}, \mathrm{d}, \mathcal{J}=8.8 \mathrm{~Hz}, \mathrm{H}-5), 8.03$ $(1 \mathrm{H}, \mathrm{d}, \mathcal{f}=9.2 \mathrm{~Hz}, \mathrm{H}-9), 7.86(1 \mathrm{H}, \mathrm{d}, \mathrm{br}, \mathcal{F}=8.0 \mathrm{~Hz}, \mathrm{H}-8), 7.74(1 \mathrm{H}$, $\mathrm{td}, \mathcal{F}=8.4,1.6 \mathrm{~Hz}, \mathrm{H}-7), 7.59(1 \mathrm{H}, \mathrm{td}, \mathcal{f}=7.6,1.6 \mathrm{~Hz}, \mathrm{H}-6), 7.34$ $(1 \mathrm{H}, \mathrm{d}, \quad \mathcal{f}=9.0 \mathrm{~Hz}, \mathrm{H}-10), 6.92(1 \mathrm{H}, \mathrm{s}, \mathrm{H}-3), 6.87(2 \mathrm{H}, \mathrm{d}$, $\mathcal{f}=10.2 \mathrm{~Hz}, \mathrm{H}-2^{\prime}$ and $\left.\mathrm{H}-6^{\prime}\right), 6.55\left(2 \mathrm{H}, \mathrm{d}, \mathcal{f}=10.4 \mathrm{~Hz}, \mathrm{H}-3^{\prime}\right.$ and H-5'), $3.54\left(2 \mathrm{H}, \mathrm{t}, \mathcal{f}=6.2 \mathrm{~Hz}, \mathrm{H}-1^{\prime \prime}\right), 1.65\left(2 \mathrm{H}, \mathrm{m}, \mathcal{f}=7.0 \mathrm{~Hz}, \mathrm{H}-2^{\prime \prime}\right)$, $1.45\left(2 \mathrm{H}, \mathrm{m}, \mathcal{f}=7.3 \mathrm{~Hz}, \mathrm{H}-3^{\prime \prime}\right), 0.95\left(3 \mathrm{H}, \mathrm{t}, \mathcal{f}=7.4 \mathrm{~Hz}, \mathrm{H}-4^{\prime \prime}\right)$ (Figures S52, S53 and S54); ${ }^{13} \mathrm{C}$ NMR (CDCl3) $\delta 184.3\left(\mathrm{C}-4^{\prime}\right), 179.5$ (C-4), 160.1 (C-2), 156.9 (C-10a), 145.9 (C-2' and C-6'), $132.2\left(\mathrm{C}-3^{\prime}\right.$ and C-5'), 135.3, 130.2, 129.9, 129.0, 127.8, 126.6, 126.4 (C-4b, C5, C-6, C-7, C-8, C-8a, C-9), 116.9 (C-10), 116.9 (C-4a), 112.1 (C-3), $73.8\left(\mathrm{C}^{\prime} 1^{\prime}\right), 64.6\left(\mathrm{C}-1^{\prime \prime}\right), 31.7\left(\mathrm{C}-2^{\prime \prime}\right), 18.8\left(\mathrm{C}-3^{\prime \prime}\right), 13.4\left(\mathrm{C}-4^{\prime \prime}\right)$; ESI-MS $(\mathrm{m} / z, \%): 383\left(\mathrm{M}^{+}+\mathrm{Na}, 100\right), 361\left(\mathbf{M}^{+}+\mathrm{H}, 65\right) ; \mathrm{HRMS} \mathrm{C}_{23} \mathrm{H}_{20} \mathrm{O}_{4} \mathrm{Na}$, calculated 383.1259; found 383.1258.

3-(1-Allyloxy-4-oxocyclohexa-2,5-dienyl)-1H-benzo [f] chromen-1-one (17). Yield: $51 \% \quad(30.5 \mathrm{mg})$; NP-HPLC: 6.650 min (Figure S19), purity: $98.0 \%$; transparent crystals, mp: $148-150^{\circ} \mathrm{C},{ }^{1} \mathrm{H}$ NMR $\left(\mathrm{CDCl}_{3}\right) \delta 9.96(1 \mathrm{H}, \mathrm{d}, \mathcal{J}=8.2 \mathrm{~Hz}, \mathrm{H}-5)$, $8.03(1 \mathrm{H}, \mathrm{d}, \mathcal{f}=8.8 \mathrm{~Hz}, \mathrm{H}-9), 7.86(1 \mathrm{H}, \mathrm{d}, \mathrm{br}, \mathcal{f}=8.2 \mathrm{~Hz}, \mathrm{H}-8)$, $7.74(1 \mathrm{H}, \mathrm{td}, \mathcal{f}=8.4,1.4 \mathrm{~Hz}, \mathrm{H}-7), 7.60(1 \mathrm{H}, \mathrm{td}, \mathcal{f}=7.6,1.0 \mathrm{~Hz}$, H-6), $7.35(1 \mathrm{H}, \mathrm{d}, \mathcal{f}=9.2 \mathrm{~Hz}, \mathrm{H}-10), 6.94(1 \mathrm{H}, \mathrm{s}, \mathrm{H}-3), 6.91(2 \mathrm{H}$, d, $\mathcal{f}=11.0 \mathrm{~Hz}, \mathrm{H}-2^{\prime}$ and $\left.\mathrm{H}-6^{\prime}\right), 6.57\left(2 \mathrm{H}, \mathrm{d}, \mathcal{f}=10.2 \mathrm{~Hz}, \mathrm{H}-3^{\prime}\right.$ and $\left.\mathrm{H}^{-} 5^{\prime}\right), 5.95\left(1 \mathrm{H}, \mathrm{m}, \mathcal{J}=5.0 \mathrm{~Hz}, \mathrm{H}-2^{\prime \prime}\right), 5.38(1 \mathrm{H}, \mathrm{dd}, \mathcal{F}=18.8$, $1.6 \mathrm{~Hz}, \mathrm{H}-3^{\prime \prime}$, cis), 5.25 (1H, dd, $\mathcal{f}=10.4,1.6 \mathrm{~Hz}, \mathrm{H}-3^{\prime \prime}$, trans), $4.09\left(2 \mathrm{H}, \mathcal{f}=5.6 \mathrm{~Hz}, \mathrm{H}-1^{\prime \prime}\right)$ (Figures S55, S56 and S57); ${ }^{13} \mathrm{C}$ NMR $(\mathrm{CDCl} 3) \delta 184.1\left(\mathrm{C}^{\prime} 4^{\prime}\right), 179.4(\mathrm{C}-4), 160.6(\mathrm{C}-2), 156.9$ (C-10a), $145.3\left(\mathrm{C}-2^{\prime}\right.$ and $\left.\mathrm{C}-6^{\prime}\right), 132.4\left(\mathrm{C}-3^{\prime}\right.$ and $\left.\mathrm{C}-5^{\prime}\right), 135.3,130.2,129.9$, 129.0, 127.8, 126.6, 126.4 (C-4b, C-5, C-6, C-7, C-8, C-8a, C-9), 116.9 (C-10), 116.9 (C-4a), 112.2 (C-3), $74.1\left(\mathrm{C}-1^{\prime}\right), 65.7\left(\mathrm{C}-1^{\prime \prime}\right)$, $133.2\left(\mathrm{C}_{-2} 2^{\prime \prime}\right), 117.2\left(\mathrm{C}-3^{\prime \prime}\right)$; ESI-MS ( $\left./ 2 / z, \%\right): 367\left(\mathbf{M}^{+}+\mathrm{Na}, 100\right)$, $345\left(\mathrm{M}^{+}+\mathrm{H}, 65\right)$; HRMS $\mathrm{C}_{22} \mathrm{H}_{16} \mathrm{O}_{4} \mathrm{Na}$, calculated 367.0946; found 367.0949; X-ray data are shown in Figure S3.

3-(1-(Prop-2-ynoxy)-4-oxocyclohexa-2,5-dienyl)-1H-benzo [f]chromen-1-one (18). Yield: 45\% (26.7 mg), NP-HPLC: $9.527 \mathrm{~min}$ (Figure S20), purity: 99.6\%; transparent crystals, mp: $142-145^{\circ} \mathrm{C},{ }^{1} \mathrm{H}$ NMR $\left(\mathrm{CDCl}_{3}\right) \delta 9.95(1 \mathrm{H}, \mathrm{d}, \mathcal{f}=7.4 \mathrm{~Hz}, \mathrm{H}-5)$, $8.04(1 \mathrm{H}, \mathrm{d}, \mathcal{J}=9.2 \mathrm{~Hz}, \mathrm{H}-9), 7.87(1 \mathrm{H}, \mathrm{d}, \mathrm{br}, \mathcal{J}=8.0 \mathrm{~Hz}, \mathrm{H}-8)$, $7.74(1 \mathrm{H}, \mathrm{td}, \mathcal{f}=8.4,1.6 \mathrm{~Hz}, \mathrm{H}-7), 7.60(1 \mathrm{H}, \mathrm{td}, \mathcal{f}=8.2,1.0 \mathrm{~Hz}$, H-6), $7.37(1 \mathrm{H}, \mathrm{d}, \mathcal{f}=9.2 \mathrm{~Hz}, \mathrm{H}-10), 6.97(2 \mathrm{H}, \mathrm{d}, \mathcal{f}=10.4 \mathrm{~Hz}$, $\mathrm{H}-2^{\prime}$ and H-6'), 6.91 (1H, s, H-3), $6.59\left(2 \mathrm{H}, \mathrm{d}, \mathcal{f}=10.4 \mathrm{~Hz}, \mathrm{H}-3^{\prime}\right.$ \left. and ${\mathrm{H}-5^{\prime}}^{\prime}\right), 4.26\left(2 \mathrm{H}, \mathrm{d}, \mathcal{F}=2.6 \mathrm{~Hz}, \mathrm{H}-\mathrm{1}^{\prime \prime}\right), 2.55(1 \mathrm{H}, \mathrm{t}, \mathcal{F}=2.4 \mathrm{~Hz}$, H-3") (Figures S58, S59 and S60); ${ }^{13} \mathrm{C}$ NMR (CDCl3) $\delta 183.9(\mathrm{C}-$ $\left.4^{\prime}\right), 179.3$ (C-4), 159.9 (C-2), 156.9 (C-10a), 144.2 (C-2' and C$\left.6^{\prime}\right), 132.6\left(\mathrm{C}_{-1} 3^{\prime}\right.$ and $\left.\mathrm{C}-5^{\prime}\right), 135.4,130.2,129.8,129.1,127.8$, 126.6, 126.5 (C-4b, C-5, C-6, C-7, C-8, C-8a, C-9), 116.8 (C-10), $116.8(\mathrm{C}-4 \mathrm{a}), 112.2$ (C-3), $74.6\left(\mathrm{C}-1^{\prime}\right), 53.2\left(\mathrm{C}-1^{\prime \prime}\right), 75.7(\mathrm{C}-$ $\left.3^{\prime \prime}\right), 79.0$ (C-2"); ESI-MS ( $\left.\mathrm{m} / \mathrm{z}, \quad \%\right)$ : $365\left(\mathrm{M}^{+}+\mathrm{Na}, 100\right), 343$ $\left(\mathrm{M}^{+}+\mathrm{H}, 52\right)$; HRMS $\mathrm{C}_{22} \mathrm{H}_{14} \mathrm{O}_{4} \mathrm{Na}$, calculated 365.0790; found 365.0788 .

\section{Supporting Information}

Figure $\mathbf{S 1} \mathrm{X}$-ray diffraction data for compound 8. CCDC 762721; formula: $\mathrm{C}_{18} \mathrm{H}_{14} \mathrm{O}_{6}$; unit cell parameters: a 6.9461(20) b 30.2804(66) c 7.6340(13) beta 93.752(19) space group P21/c.

(TIF)

Figure S2 X-ray diffraction data for compound 14 . CCDC 762722 ; formula: $\mathrm{C}_{22} \mathrm{H}_{18} \mathrm{O}_{4}$; unit cell parameters: a 7.8452(8) b 25.285(5) c 18.144(2) beta 96.096(9) space group P21/c. (TIF)

Figure S3 X-ray diffraction data for compound 17. CCDC 762723; formula: $\mathrm{C}_{22} \mathrm{H}_{16} \mathrm{O}_{4}$; unit cell parameters: a 7.9122(8) b 24.817(5) c 17.8259(16) beta 96.380(8) space group P21/c. (TIF)

Figure S4 RP-HPLG chromatogram of compound 1. Solvent: $35 \% \mathrm{v} / \mathrm{v}$ aqueous $\mathrm{MeOH}$ increasing to $80 \%$ in $10 \mathrm{~min}$ and subsequently changing back to $35 \%$; flow rate: $1 \mathrm{~mL} / \mathrm{min}$, $\lambda=245 \mathrm{~nm}$

(TIF)

Figure S5 RP-HPLC chromatogram of compound 1". Solvent: $35 \% \mathrm{v} / \mathrm{v}$ aqueous $\mathrm{MeOH}$ increasing to $80 \%$ in $10 \mathrm{~min}$ and subsequently changing back to $35 \%$; flow rate: $1 \mathrm{~mL} / \mathrm{min}$, $\lambda=245 \mathrm{~nm}$

(TIF)

Figure S6 RP-HPLG chromatogram of compound 3. Solvent: $55 \% \mathrm{v} / \mathrm{v}$ aqueous $\mathrm{MeOH}$ increasing to $80 \%$ in $10 \mathrm{~min}$ and subsequently changing back to $55 \%$; flow rate: $1 \mathrm{~mL} / \mathrm{min}$, $\lambda=245 \mathrm{~nm}$

(TIF)

Figure S7 RP-HPLG chromatogram of compound 4. Solvent: $55 \% \mathrm{v} / \mathrm{v}$ aqueous $\mathrm{MeOH}$ increasing to $80 \%$ in $10 \mathrm{~min}$ and subsequently changing back to $55 \%$; flow rate: $1 \mathrm{~mL} / \mathrm{min}$, $\lambda=245 \mathrm{~nm}$

(TIF)

Figure S8 RP-HPLC chromatogram of compound 5. Solvent: $55 \% \mathrm{v} / \mathrm{v}$ aqueous $\mathrm{MeOH}$ increasing to $80 \%$ in $10 \mathrm{~min}$ and subsequently changing back to $55 \%$; flow rate: $1 \mathrm{~mL} / \mathrm{min}$, $\lambda=245 \mathrm{~nm}$

(TIF)

Figure S9 RP-HPLC chromatogram of compound 6 . Solvent: $55 \% \mathrm{v} / \mathrm{v}$ aqueous $\mathrm{MeOH}$ increasing to $80 \%$ in $10 \mathrm{~min}$ and subsequently changing back to $55 \%$; flow rate: $1 \mathrm{~mL} / \mathrm{min}$, $\lambda=245 \mathrm{~nm}$

(TIF)

Figure S10 RP-HPLC chromatogram of compound 7. Solvent: $55 \% \mathrm{v} / \mathrm{v}$ aqueous $\mathrm{MeOH}$ increasing to $80 \%$ in $10 \mathrm{~min}$ and subsequently changing back to $55 \%$; flow rate: $1 \mathrm{~mL} / \mathrm{min}$, $\lambda=245 \mathrm{~nm}$

(TIF)

Figure S11 RP-HPLC chromatogram of compound 8. Solvent: $55 \% \mathrm{v} / \mathrm{v}$ aqueous $\mathrm{MeOH}$ increasing to $80 \%$ in $10 \mathrm{~min}$ and subsequently changing back to $55 \%$; flow rate: $1 \mathrm{~mL} / \mathrm{min}$, $\lambda=245 \mathrm{~nm}$

(TIF)

Figure S12 RP-HPLC chromatogram of compound 9. Solvent: $55 \% \mathrm{v} / \mathrm{v}$ aqueous $\mathrm{MeOH}$ increasing to $80 \%$ in $10 \mathrm{~min}$ and subsequently changing back to $55 \%$; flow rate: $1 \mathrm{~mL} / \mathrm{min}$, $\lambda=245 \mathrm{~nm}$

(TIF) 
Figure S13 NP-HPLC chromatogram of compound 11. Solvent: $5 \% \mathrm{v} / \mathrm{v}$ isopropanol in dichloromethane increasing to $17 \%$ in $12 \mathrm{~min}$; flow rate: $1 \mathrm{~mL} / \mathrm{min}, \lambda=245 \mathrm{~nm}$

(TIF)

Figure S14 NP-HPLG chromatogram of compound 12. Solvent: $5 \% \mathrm{v} / \mathrm{v}$ isopropanol in dichloromethane increasing to $17 \%$ in $12 \mathrm{~min}$; flow rate: $1 \mathrm{~mL} / \mathrm{min}, \lambda=245 \mathrm{~nm}$ (TIF)

Figure S15 NP-HPLG chromatogram of compound 13. Solvent: $5 \% \mathrm{v} / \mathrm{v}$ isopropanol in dichloromethane increasing to $17 \%$ in $12 \mathrm{~min}$; flow rate: $1 \mathrm{~mL} / \mathrm{min}, \lambda=245 \mathrm{~nm}$

(TIF

Figure S16 NP-HPLC chromatogram of compound 14. Solvent: $5 \% \mathrm{v} / \mathrm{v}$ isopropanol in dichloromethane increasing to $17 \%$ in $12 \mathrm{~min}$; flow rate: $1 \mathrm{~mL} / \mathrm{min}, \lambda=245 \mathrm{~nm}$

(TIF)

Figure S17 NP-HPLC chromatogram of compound 15. Solvent: $5 \% \mathrm{v} / \mathrm{v}$ isopropanol in dichloromethane increasing to $17 \%$ in $12 \mathrm{~min}$; flow rate: $1 \mathrm{~mL} / \mathrm{min}, \lambda=245 \mathrm{~nm}$

(TIF)

Figure S18 NP-HPLC chromatogram of compound 16. Solvent: $5 \% \mathrm{v} / \mathrm{v}$ isopropanol in dichloromethane increasing to $17 \%$ in $12 \mathrm{~min}$; flow rate: $1 \mathrm{~mL} / \mathrm{min}, \lambda=245 \mathrm{~nm}$ (TIF)

Figure S19 NP-HPLC chromatogram of compound 17. Solvent: $5 \% \mathrm{v} / \mathrm{v}$ isopropanol in dichloromethane increasing to $17 \%$ in $12 \mathrm{~min}$; flow rate: $1 \mathrm{~mL} / \mathrm{min}, \lambda=245 \mathrm{~nm}$

(TIF)

Figure S20 NP-HPLG chromatogram of compound 18. Solvent: $5 \% \mathrm{v} / \mathrm{v}$ isopropanol in dichloromethane increasing to $17 \%$ in $12 \mathrm{~min}$; flow rate: $1 \mathrm{~mL} / \mathrm{min}, \lambda=245 \mathrm{~nm}$

(TIF)

Figure S21 $400 \mathrm{MHz}^{1} \mathrm{H}$ NMR spectrum of compound 1". (TIF)

Figure S22 Zoom of $400 \mathrm{MHz}^{1} \mathrm{H}$ NMR spectrum of compound 1".

(TIF)

Figure S23 $200 \mathrm{MHz}^{1} \mathrm{H}$ NMR spectrum of compound 3 before crystallization.

(TIF)

Figure S24 Zoom of $200 \mathrm{MHz}{ }^{1} \mathrm{H}$ NMR spectrum of compound 3 before crystallization.

(TIF)

Figure S25 $200 \mathrm{MHz}{ }^{1} \mathrm{H}$ NMR spectrum of compound 4 before crystallization.

(TIF)

Figure S26 Zoom of $200 \mathrm{MHz}{ }^{1} \mathrm{H}$ NMR spectrum of compound 4 before crystallization.

(TIF)

Figure S27 Zoom of $200 \mathrm{MHz}{ }^{1} \mathrm{H}$ NMR spectrum of compound 4 before crystallization.

(TIF)

Figure S28 $200 \mathrm{MHz}{ }^{1} \mathrm{H}$ NMR spectrum of compound 5 before crystallization.

(TIF)
Figure S29 Zoom of $200 \mathrm{MHz}{ }^{1} \mathrm{H}$ NMR spectrum of compound 5 before crystallization.

(TIF)

Figure S30 Zoom of $200 \mathrm{MHz}{ }^{1} \mathrm{H}$ NMR spectrum of compound 5 before crystallization.

(TIF)

Figure S31 $200 \mathrm{MHz}^{1} \mathrm{H}$ NMR spectrum of compound 6 before crystallization.

(TIF)

Figure S32 Zoom of $200 \mathrm{MHz}{ }^{1} \mathrm{H}$ NMR spectrum of compound 6 before crystallization.

(TIF)

Figure S33 Zoom of $200 \mathrm{MHz}{ }^{1} \mathrm{H}$ NMR spectrum of compound 6 before crystallization.

(TIF)

Figure S34 Zoom of $200 \mathrm{MHz}^{1} \mathrm{H}$ NMR spectrum of compound 6 before crystallization.

(TIF)

Figure S35 $200 \mathrm{MHz}^{1} \mathrm{H}$ NMR spectrum of compound 7 before crystallization.

(TIF)

Figure S36 Zoom of $200 \mathrm{MHz}^{1} \mathrm{H}$ NMR spectrum of compound 7 before crystallization.

(TIF)

Figure S37 Zoom of $200 \mathrm{MHz}{ }^{1} \mathrm{H}$ NMR spectrum of compound 7 before crystallization.

(TIF)

Figure S38 $200 \mathrm{MHz}^{1} \mathrm{H}$ NMR spectrum of compound 8 before crystallization.

(TIF)

Figure S39 Zoom of $200 \mathrm{MHz}{ }^{1} \mathrm{H}$ NMR spectrum of compound 8 before crystallization.

(TIF)

Figure S40 $200 \mathrm{MHz}^{1} \mathrm{H}$ NMR spectrum of compound 9 before crystallization.

(TIF)

Figure S41 Zoom of $200 \mathrm{MHz}{ }^{1} \mathrm{H}$ NMR spectrum of compound 9 before crystallization.

(TIF)

Figure S42 $400 \mathrm{MHz}^{1} \mathrm{H}$ NMR spectrum of compound 9 before crystallization.

(TIF)

Figure S43 $200 \mathrm{MHz}^{1} \mathrm{H}$ NMR spectrum of compound 13 before crystallization.

(TIF)

Figure S44 Zoom of $200 \mathrm{MHz}{ }^{1} \mathrm{H}$ NMR spectrum of compound 13 before crystallization.

(TIF)

Figure S45 Zoom of $200 \mathrm{MHz}{ }^{1} \mathrm{H}$ NMR spectrum of compound 13 before crystallization.

(TIF)

Figure S46 $200 \mathrm{MHz}^{1} \mathrm{H}$ NMR spectrum of compound 14 before crystallization.

(TIF)

Figure S47 Zoom of $200 \mathrm{MHz}{ }^{1} \mathrm{H}$ NMR spectrum of compound 14 before crystallization.

(TIF) 
Figure S48 Zoom of $200 \mathrm{MHz}{ }^{1} \mathrm{H}$ NMR spectrum of compound 14 before crystallization.

(TIF)

Figure S49 $200 \mathrm{MHz}^{1} \mathrm{H}$ NMR spectrum of compound 15 before crystallization.

(TIF)

Figure S50 Zoom of $200 \mathrm{MHz}{ }^{1} \mathrm{H}$ NMR spectrum of compound 15 before crystallization.

(TIF)

Figure S51 Zoom of $200 \mathrm{MHz}{ }^{1} \mathrm{H}$ NMR spectrum of compound 15 before crystallization.

(TIF)

Figure S52 $200 \mathrm{MHz}^{1} \mathrm{H}$ NMR spectrum of compound 16 before crystallization.

(TIF)

Figure S53 Zoom of $200 \mathrm{MHz}^{1} \mathrm{H}$ NMR spectrum of compound 16 before crystallization.

(TIF)

Figure S54 Zoom of $200 \mathrm{MHz}{ }^{1} \mathrm{H}$ NMR spectrum of compound 16 before crystallization.

(TIF)

Figure S55 $200 \mathrm{MHz}{ }^{1} \mathrm{H}$ NMR spectrum of compound 17 before crystallization.

(TIF)

\section{References}

1. Di Carlo G, Mascolo N, Izzo AA, Capasso F (1999) Flavonoids: Old and new aspects of a class of natural therapeutic drugs. Life Sci 65: 337-353.

2. Le Marchand L (2002) Cancer preventive effects of flavonoids-a review. Biomed Pharmacother 56: 296-301.

3. Kale A, Gawande S, Kotwal S (2008) Cancer phytotherapeutics: role for flavonoids at the cellular level. Phytother Res 22: 567-577.

4. Kandaswami G, Lee LT, Lee PP, Hwang.JJ, Ke FC, et al. (2005) The antitumor activities of flavonoids. In Vivo 19: 895-909.

5. Li Y, Fang H, Xu W (2007) Recent advance in the research of flavonoids as anticancer agents. Mini Rev Med Chem 7: 663-678.

6. Lin AS, Chang FR, Wu CG, Liaw CG, Wu YG (2005) New Cytotoxic Flavonoids from Thelypteris torresiana. Planta Med 71: 867-870.

7. Patel D, Shukla S, Gupta S (2007) Apigenin and cancer chemoprevention: progress, potential and promise (review). Int J Oncol 30: 233-245.

8. Liu LZ, Fang J, Zhou Q Hu X, Shi X, et al. (2005) Apigenin Inhibits Expression of Vascular Endothelial Growth Factor and Angiogenesis in Human Lung Cancer Cells: Implication of Chemoprevention of Lung Cancer. Mol Pharmacol 68: 635-643

9. Chang HL, Wu YC, Su JH, Yeh YT, Yuan SSF (2008) Protoapigenone, a Novel Flavonoid, Induces Apoptosis in Human Prostate Cancer Cells through Activation of p38 Mitogen-Activated Protein Kinase and c-Jun $\mathrm{NH}_{2}$-Terminal Kinase 1/2. J Pharmacol Exp Ther 325: 841-849.

10. Chang HL, Su JH, Yeh YT, Lee YC, Chen HM, et al. (2008) Protoapigenone, a novel flavonoid, inhibits ovarian cancer cell growth in vitro and in vivo. Cancer Lett 267: 85-95.

11. Chiu CC, Chang HW, Chuang DW, Chang FR, Chang YC, et al. (2009) Fern plant-derived protoapigenone leads to DNA damage, apoptosis, and $\mathrm{G}(2) / \mathrm{m}$ arrest in lung cancer cell line H1299. DNA Cell Biol 28: 501-506.
Figure S56 Zoom of $200 \mathrm{MHz}{ }^{1} \mathrm{H}$ NMR spectrum of compound 17 before crystallization.

(TIF)

Figure S57 Zoom $200 \mathrm{MHz}{ }^{1} \mathrm{H}$ NMR spectrum of compound 17 before crystallization.

(TIF)

Figure S58 $200 \mathrm{MHz}^{1} \mathrm{H}$ NMR spectrum of compound 18 before crystallization.

(TIF)

Figure S59 Zoom of $200 \mathrm{MHz}^{1} \mathrm{H}$ NMR spectrum of compound 18 before crystallization.

(TIF)

Figure S60 Zoom of $200 \mathrm{MHz}{ }^{1} \mathrm{H}$ NMR spectrum of compound 18 before crystallization.

(TIF)

\section{Author Contributions}

Conceived and designed the experiments: AH DWC FRC YGW. Performed the experiments: AH DWC BD. Analyzed the data: AH FRC. Contributed reagents/materials/analysis tools: AH DWG CLL HCW CCW FRG YCW. Wrote the paper: AH. Performed X-ray experiments: MYC.
12. Lin AS, Nakagawa-Goto K, Chang FR, Yu D, Morris-Natschke SL, et al. (2007) First Total Synthesis of Protoapigenone and Its Analogues as Potent Cytotoxic Agents. J Med Chem 50: 3921-3927.

13. Felpin FX (2007) Oxidation of 4-arylphenol trimethylsilyl ethers to p-arylquinols using hypervalent iodine(III) reagents. Tetrahedron Lett 48: 409-412.

14. Wells G, Berry JM, Bradshaw TD, Burger AM, Seaton A, et al. (2003) 4Substituted 4-Hydroxycyclohexa-2,5-dien-1-ones with Selective Activities against Colon and Renal Cancer Cell Lines. J Med Chem 46: 532-541.

15. Zhdankin VV (2009) Hypervalent iodine(III) reagents in organic synthesis. ARKIVOC (i). pp 1-62.

16. Quideau S, Pouységu L, Deffieux D (2008) Oxidative Dearomatization of Phenols: Why, How and What For? Synlett 4: 467-495.

17. Hamamoto H, Anilkumar G, Tohma H, Kita Y (2002) A Novel and Useful Oxidative Intramolecular Coupling Reaction of Phenol Ether Derivatives on Treatment with a Combination of Hypervalent Iodine (III) Reagent and Heteropoly Acid. Chem Eur J 8: 5377-5383.

18. Favaro G, Clementi C, Romani A, Vickackaite V (2007) Acidichromism and Ionochromism of Luteolin and Apigenin, the Main Components of the Naturally Occurring Yellow Weld: A Spectrophotometric and Fluorimetric Study. J Fluoresc 17: 707-714.

19. Wagner H, Chari VM, Sonnenblicher J (1976) Carbon-13 NMR spectra of naturally occurring flavonoids. Tetrahedron Lett 21: 1799-1802.

20. Khokhrina TA, Peshkova VA, Glyzin VI (1973) Flavonoids from Phlomis tuberosa. Khim Prir Soedin 6: 802

21. Mosmann T (1983) Rapid Colorimetric Assay for Cellular Growth and Survival: Application to Proliferation and Cytotoxicity Assays. J Immunol Methods 65: $55-63$. 


\title{
In Vitro Cytotoxic Activity of Novel Protoflavone Analogs - Selectivity Towards a Multidrug Resistant Cancer Cell Line
}

\author{
B. DANKO ${ }^{1}$, A. MARTINS ${ }^{1,2}$, D.W. CHUANG ${ }^{3}$, H.C. WANG $^{3}$, L. AMARAL ${ }^{4 \dagger}$, \\ J. MOLNÁR ${ }^{2 \dagger}$, F.R. CHANG ${ }^{3,5,6}$, Y.C. WU ${ }^{7,8,9}$ and A. HUNYADI ${ }^{1+}$ \\ ${ }^{1}$ Institute of Pharmacognosy, University of Szeged, Szeged, Hungary; \\ ${ }^{2}$ Department of Medical Microbiology and Immunobiology, \\ Faculty of Medicine, University of Szeged, Szeged, Hungary; \\ ${ }^{3}$ Graduate Institute of Natural Products, Kaohsiung Medical University, Kaohsiung, Taiwan, R.O.C.; \\ ${ }^{4}$ Group of Mycobacteria, Unit of Microbiology, Institute of Hygiene and Tropical Medicine, \\ Universidade Nova de Lisboa, CMDT, Portugal; \\ ${ }^{5}$ Cancer Center, Kaohsiung Medical University Hospital, Kaohsiung, Taiwan, R.O.C.; \\ ${ }^{6} R \& D$ Center of Chinese Herbal Medicines \& New Drugs, College of Pharmacy, \\ Kaohsiung Medical University, Kaohsiung, Taiwan, R.O.C.; \\ ${ }^{7}$ School of Chinese Medicine, College of Chinese Medicine, China Medical University, Taichung, Taiwan, R.O.C., \\ ${ }^{8}$ Natural Medicinal Products Research Center, China Medical University Hospital, Taichung, Taiwan, R.O.C.; \\ ${ }^{9}$ Center for Molecular Medicine, China Medical University Hospital, Taichung, Taiwan, R.O.C.
}

\begin{abstract}
Background: Protoapigenone (PA), a natural flavonoid possessing an unusual p-quinol moiety on its B ring, is a prospective novel lead compound against cancer currently in development, together with WYC0209, a potent synthetic PA analog. Structure activity relationships (SAR) concerning different 1'-O-alkyl side-chains were also studied on two sets of derivatives. Materials and Methods: Fifteen 1'-O-alkyl protoflavone derivatives were synthesized from genkwanin or 4'-hydroxy-6-methylflavone, thirteen of which are new compounds. All compounds were tested for their cytotoxic
\end{abstract}

$\dagger$ COST Action BM0701 Antibiotic Transport and Efflux: New Strategies to Combat Bacterial Resistance (ATENS) of the European Commission, Brussels, Belgium.

$¥$ COST Action CM0804 (Chemical Biology with Natural Products) of the European Commission, Brussels, Belgium.

Correspondence to: Attila Hunyadi, Institute of Pharmacognosy, Faculty of Pharmacy, University of Szeged, 6720 Szeged, Eötvös Str. 6, Hungary. Tel: +36 62546456, Fax: +36 62545704, e-mail: hunyadi.a@pharm.u-szeged.hu; and Yang-Chang Wu, Graduate Institute of Integrated Medicine, College of Chinese Medicine, China Medical University, 404 Taichung, Hsueh-Shih Rd. 91, Taiwan, R.O.C. Tel: +886 422057153, Fax: +886 422060248, e mail: yachwu@mail.cmu.edu.tw

Key Words: Protoflavone, protoapigenone, WYC0209, 6methylflavone, selective cytotoxicity, MDR, ABCB1, P-glycoprotein, P-gp. effect on four human cancer cell lines, such as HepG2 and Hep3B (hepatic), A549 (lung) and MDA-MB-231 (breast) cell lines, with doxorubicin as a positive control. All compounds, as well as PA, WYCO209 and fourteen of their previously reported analogs were also tested on a multidrug-resistant (MDR) sub-cell line of L5178 mouse T-cell lymphoma and on its parental counterpart (PAR). Results: In general, derivatives bearing a free hydroxyl group at C-1' exerted the strongest activities, while C-1'-substituted compounds were found to be much weaker. Derivatives of 6-methylflavone exhibited mild, but statistically significant selectivity towards the MDR cell line. Conclusion: The results are in agreement with our previous findings for fundamental SAR of protoflavones. 6Methylated protoflavones may serve as valuable leads for developing selective compounds against MDR cancer. Identical activity of other derivatives on the PAR and MDR cell lines suggests that cancer cells cannot exhibit resistance to protoflavones by $A B C B 1$ efflux pump overexpression.

Flavonoids have long been proposed to exert various bioactivities interfering with cancer cells on several levels, including those connected to their antioxidant and chemopreventive effects, and, for certain derivatives, specific anticancer activities have also been described (1-4).

Protoapigenone is an oxidized derivative of the common flavonoid apigenin, expressing an unusual $p$-quinol moiety in its B-ring; the structures of these two compounds as well as that of WYC0209, the strongest synthetic derivative of protoapigenone obtained to date, are shown in Figure 1. 

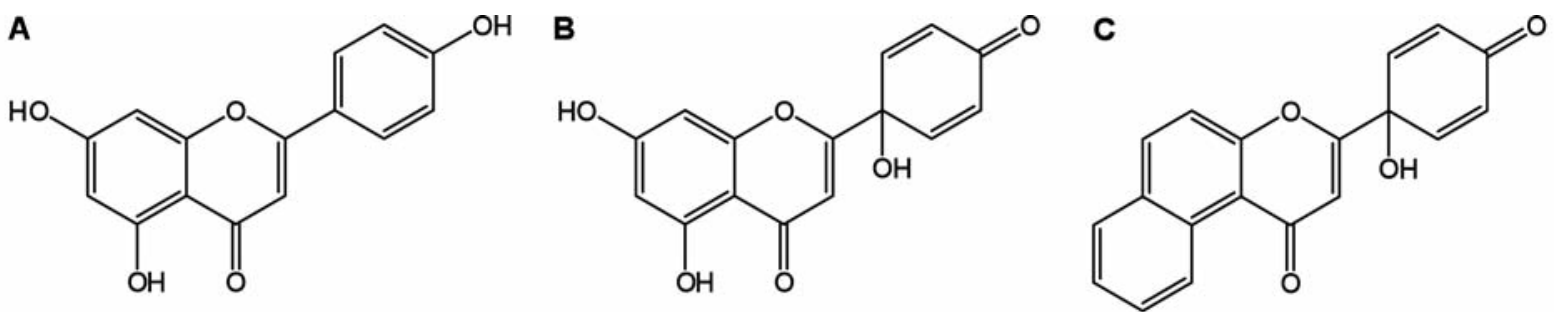

Figure 1. Structures of apigenin (A), protoapigenone (B) and WYC0209, currently the strongest synthetic protoflavone derivative known (C).

Isolated for the first time from the fern Thelypteris torresiana, protoapigenone was recently discovered as a promising, natural anticancer agent (5) based on its strong antitumor activity against several cancer cell lines of different origin both in vitro and in vivo $(5,6)$. It induces apoptosis through the activation of $\mathrm{p} 38$ mitogen-activated protein kinase and c-Jun $\mathrm{NH}_{2}$-terminal kinase $1 / 2$ (7) resulting in a $G(2) / m$ cell cycle arrest (8), in which the role of oxidative stress and glutathione- $S$-transferase $\pi$ inhibition was also suggested (9). Most recently, a unique mechanism was found for the anticancer action of protoapigenone and WYC0209: these flavonoids were found to affect the ataxia telangiectasia-mutated and Rad3-related (ATR) signaling pathway both in vitro and in vivo (10). This pathway plays an essential role in maintaining genomic integrity, and it is considered an attractive target for the development of a new class of anticancer drugs (11). Interestingly, as well as its cancer-related effects, protoapigenone was also found to inhibit the lytic cycle of Epstein-Barr virus (12), a wellknown risk factor for several malignant diseases.

After the successful total synthesis of protoapigenone and several of its derivatives (13), a very simple semi-synthetic way to obtain this compound directly from apigenin was also reported by our group (14). This work also resulted in the production of several 1'- $O$-alkyl derivatives of both protoapigenone and WYC0209, revealing new side-chaindependent structure activity relationships within the protoapigenone derivatives but not within those of the WYC0209. Although a 1'- $\mathrm{OCH}_{3}$ group markedly reduced the cytotoxic activity, increasing the length of an aliphatic side chain restored, or even increased the activity. This effect did not occur in the presence of a branching or unsaturated side chain (14). As a direct continuation of this work, here we report the synthesis and cytotoxicity evaluation of several new protoflavones towards various human cancer cell lines and two mouse lymphoma cell lines: a parental (susceptible, PAR) cell line and its multidrug resistant (MDR) sub-cell line expressing the human ATP Biding Cassette (ABC) B1 efflux pump, which, as the most prevalent $\mathrm{ABC}$ transporter, is among the major reasons for failure of cancer chemotherapy (15).

\section{Materials and Methods}

Protoflavones. Compounds 1-15 were synthesized from 4'hydroxyflavones (genkwanin, 4'-hydroxy-6-methylflavone and 4'hydroxy-6-methoxyflavone) obtained from Indofine Chemical Company Ltd. (Hillsborough, NJ, USA), as reported previously (14). Briefly, the starting material was dissolved in a 9:1 (v/v) mixture of acetonitrile and water or the alcohol to be coupled to $\mathrm{C}-1$ ' as an $O$ alkyl side chain, at a concentration of $1 \mathrm{mg} / \mathrm{ml}$. After this, two equivalents of [bis(trifluoroacetoxy)iodo]benzene (Sigma Aldrich, Munich, Germany) were added to the mixture, the container was sealed and 1-min microwave heating was applied at $300 \mathrm{~W}$ in a standard domestic microwave oven. After cooling, the reaction mixture was evaporated, re-dissolved in acetone and purified by rotational planar chromatography on a Chromatothron equipment (Harrison Research, Palo Alto, California, USA) with adequately chosen eluents consisting of $n$-hexane-ethyl acetate-acetone on silica. Solvents and silica were purchased from Merck (Darmstadt, Germany). Protoflavones obtained were re-crystallized from solvent mixtures similar to the eluents. Synthesis, bioactivity on the human cancer cell lines and compound characterization data for compounds 16-29, were reported previously (14).

Structure elucidation carried out by means of electrospray ionization tandem mass spectrometry (ESI-MS/MS) and nuclear magnetic resonance (NMR) spectroscopy. MS and MS/MS spectra were taken on an API 2000 triple-quadrupole tandem mass spectrometer (AB SCIEX, Foster City, CA, USA) in ESI+ mode. NMR spectra were obtained on a Bruker Avance DRX-500 spectrometer in acetone- $d_{6}$, methanol- $d_{4}$, or chloroform- $d_{l}$.

Cell lines. Human cell lines: Human liver (HepG2 and Hep3B), lung (A549) and breast (MDA-MB-231) cancer cell lines were obtained from the American Type Culture Collection. All cell lines were propagated in RPMI-1640 medium, supplemented with $10 \%$ $(\mathrm{v} / \mathrm{v})$ fetal bovine serum (FBS), $100 \mathrm{U} / \mathrm{ml}$ of penicillin, and 100 $\mu \mathrm{g} / \mathrm{ml}$ of streptomycin at $37^{\circ} \mathrm{C}$ in a humidified atmosphere of $5 \%$ $\mathrm{CO}_{2}$ and $95 \%$ air.

Mouse lymphoma cell lines. PAR and MDR cell lines were L5178 mouse T-cell lymphoma cells and the L5178 cells transfected with pHa MDR1/A retrovirus, respectively. MDR cell lines were selected by culturing the infected cells with $60 \mu \mathrm{g} / 1$ of colchicine (16). Both cell lines were cultured in McCoy's 5A medium, supplemented with $10 \%$ heat-inactivated horse serum, L-glutamine and antibiotics (penicillin and streptomycin), at $37^{\circ} \mathrm{C}$ and in an atmosphere with $5 \% \mathrm{CO}_{2}$. 
<smiles>[R]C1(c2cc(=O)c3c(O)cc(OC)cc3o2)C=CC(=O)C=C1</smiles>

\begin{tabular}{lc}
-R & Compound \\
\hline$-H$ & 1 \\
-Methyl & 2 \\
-Ethyl & 3 \\
-Propyl & 4 \\
-Propyl & 5 \\
-Butyl & 6 \\
-Allyl & 7 \\
-Propargyl & $\mathbf{8}$
\end{tabular}<smiles>[R]C1(c2cc(=O)c3c(O)cc(O)cc3o2)C=CC(=O)C=C1</smiles>

\begin{tabular}{lc}
- R & Compound \\
\hline$-H$ & $P A$ \\
-Methyl & 16 \\
-Ethyl & 17 \\
-Propyl & 18 \\
-Propyl & 19 \\
-Butyl & 20 \\
-Allyl & 21 \\
-Propargyl & 22
\end{tabular}

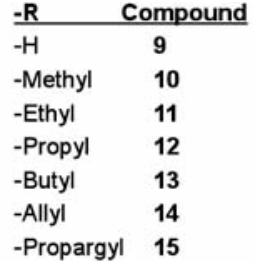

\begin{tabular}{ll}
$-R$ & Compound \\
\hline$-H$ & WYC0209 \\
-Methyl & 23 \\
-Ethyl & 24 \\
-Propyl & 25 \\
-iPropyl & 26 \\
-Butyl & 27 \\
-Allyl & 28 \\
-Propargyl & 29
\end{tabular}

Figure 2. Structures of the novel protoflavone derivatives 1-15 obtained (A), and those of the analogs of protoapigenone (16-22) and WYC0209 (2329) reported previously (14) (B). Substituents at position l'for the individual compounds are presented next to their corresponding core structures.

Cytotoxicity assay. Cell viability was measured by the $3-(4,5-$ Dimethylthiazol-2-yl)-2,5-diphenyltetrazolium bromide (MTT) colorimetric method (17). Human cancer cells were seeded at densities of 5,000 to 10,000 cells/well in 96 -well tissue culture plates. On the next day, cells were treated with $10 \mu \mathrm{l}$ of the test compounds dissolved at different concentrations for another $72 \mathrm{~h}$. After drug treatment, attached cells were incubated with MTT $(0.5 \mathrm{mg} / \mathrm{ml}$, for 1 h) and subsequently solubilized in dimethyl sulfoxide (DMSO). The absorption at $550 \mathrm{~nm}$ was then measured using a microplate reader.

In contrast to these, the non-adherent PAR and MDR cell lines were used at 20,000 cells/well without being seeded but treated immediately. The cells were incubated for $24 \mathrm{~h}$, after which MTT was added to a final concentration of $10 \%$ per well. After $4 \mathrm{~h}$ of incubation, sodium dodecyl sulfate (SDS) $10 \%$ was also added to a final concentration of 5\% per well and cells were incubated overnight. Absorption was read at 540 and $630 \mathrm{~nm}$ using an ELISA reader (Multiskan EX, Lab Systems, USA).

Each assay was performed in triplicate. In both protocols, the $\mathrm{IC}_{50}$ was recorded as the concentration of the agent that reduced cell viability by $50 \%$ under the experimental conditions, as determined by applying $\log$ (concentration) $v s$. inhibition variable slope nonlinear regression with automatic outlier elimination at $\mathrm{Q}=1.0 \%$, by using GraphPad Prism 5.0 (GraphPad Software Inc., California, USA), and $95 \%$ confidence intervals of the $\mathrm{IC}_{50}$ values were obtained from the curve fitting. Statistical evaluation of data obtained for the PAR and MDR cell lines was performed by using one-way ANOVA followed by Bonferroni's multiple comparison test; in those cases where variances were not significantly different, unpaired $t$-tests were also performed in order to test for occasional differences under less strict statistical conditions.

\section{Results and Discussion}

With a microwave-assisted oxidative de-aromatization by a common hypervalent iodine reagent, 15 protoflavones were obtained from genkwanin, 4'-hydroxy-6-methylflavone. The structure elucidation of the synthesized compounds was performed according to the following: As a first step, by means of the ESI-MS and MS/MS spectra, the expected molecular mass of each compound was confirmed. After this, analyzing the ${ }^{1} \mathrm{H}-\mathrm{NMR}$ spectrum was sufficient to unambiguously prove successful transformation: the characteristic coupling constants of $8.8 \mathrm{~Hz}$ of the two doublets present in the aromatic B-ring of the starting material (representing $\mathrm{H}-2^{\prime} / \mathrm{H}-6^{\prime}$ ' and $\mathrm{H}-3^{\prime} / \mathrm{H}-5$ ') changed to $\sim 10.0 \mathrm{~Hz}$, suggesting the formation of a symmetric cyclohexadien-1-one, and aliphatic proton signals, and their multiplicity, together with the practically unchanged A-ring signals and $\mathrm{H}-3$ singlet, proved the presence of the expected side-chain at position $\mathrm{C}^{-1}$ '; detailed data are presented in the Experimental section. Structures of the obtained compounds, as well as those of the ones previously reported (14), are presented in Figure 2. Compounds 3-15 are described here for the first time.

All derivatives were tested for their cytotoxicity towards HepG2, Hep3B, A549 and MDA-MB-231 human cancer cell lines; results are summarized in Table I.

Structure activity relationships previously reported by our group (14) were confirmed by the present experiments. The presence of 1-O-alkyl side-chain increases the $\mathrm{IC}_{50}$ in the case of derivatives of the less polar 4'-hydroxyflavones. The cytotoxic activities of compounds 10-15 were significantly weaker than that of compound $\mathbf{9}$. Interestingly, derivatives of genkwanin had a similar behavior, and cytotoxicity of these compounds was generally also not restored or increased when a 3-4 carbon aliphatic O-alkyl side-chain was introduced to C-1' [as found for protoapigenone (14)] with one single exception: compound 6 (1'-O-butyl ether) was as 
Table I. Inhibition concentration $\left(I C_{50}\right)$ values of 1-15 and, in parenthesis, the corresponding 95\% confidence intervals for the six cancer cell lines.

\begin{tabular}{|c|c|c|c|c|}
\hline & HepG2 & Нер3В & A549 & MDA-MB-231 \\
\hline \multirow[t]{2}{*}{ PA } & $3.07^{\mathrm{a}}$ & $1.21^{\mathrm{a}}$ & $11.29^{\mathrm{a}}$ & $1.35^{\mathrm{a}}$ \\
\hline & $(2.83-3.34)$ & $(1.19-1.24)$ & $(10.07-12.65)$ & $(1.17-1.55)$ \\
\hline \multirow[t]{2}{*}{ WYC0209 } & $1.06^{\mathrm{a}}$ & $0.25^{\mathrm{a}}$ & $2.19^{\mathrm{a}}$ & $0.43^{\mathrm{a}}$ \\
\hline & $(1.04-1.08)$ & $(0.20-0.30)$ & $(2.03-2.37)$ & $(0.41-0.45)$ \\
\hline \multirow[t]{2}{*}{1} & 1.86 & 0.64 & 1.77 & 0.70 \\
\hline & $(1.85-1.88)$ & $(0.55-0.73)$ & $(1.50-2.08)$ & $(0.64-0.77)$ \\
\hline \multirow[t]{2}{*}{2} & 7.65 & 1.88 & 12.09 & 2.08 \\
\hline & $(7.44-7.87)$ & $(1.55-2.28)$ & $(8.95-16.34)$ & $(1.96-2.22)$ \\
\hline \multirow[t]{2}{*}{3} & 7.27 & 1.82 & 10.97 & 2.09 \\
\hline & $(7.05-7.50)$ & $(1.73-1.91)$ & $(9.24-13.03)$ & $(1.99-2.20)$ \\
\hline \multirow[t]{2}{*}{4} & 8.69 & 1.83 & 10.27 & 2.20 \\
\hline & $(8.05-9.38)$ & $(1.33-2.52)$ & $(9.49-11.11)$ & $(2.05-2.36)$ \\
\hline 5 & $>50$ & $>50$ & $>50$ & $>50$ \\
\hline \multirow[t]{2}{*}{6} & 12.27 & 0.65 & 11.83 & 5.13 \\
\hline & $(11.70-12.87)$ & $(0.61-0.68)$ & $(10.16-13.77)$ & $(4.52-5.84)$ \\
\hline \multirow[t]{2}{*}{7} & 7.85 & 1.65 & 5.04 & 1.87 \\
\hline & $(7.66-8.04)$ & $(1.15-2.36)$ & $(3.48-7.31)$ & $(1.74-2.01)$ \\
\hline 8 & n. d. & n. d. & n. d. & n. d. \\
\hline \multirow[t]{2}{*}{9} & 2.02 & 0.54 & 2.00 & 0.61 \\
\hline & $(1.87-2.18)$ & $(0.53-0.54)$ & $(1.82-2.19)$ & $(0.57-0.65)$ \\
\hline \multirow[t]{2}{*}{10} & 9.79 & 3.18 & 15.47 & 5.46 \\
\hline & $(9.23-10.39)$ & $(2.64-3.83)$ & $(13.15-18.20)$ & $(5.12-5.82)$ \\
\hline \multirow[t]{2}{*}{11} & 8.61 & 2.17 & 7.35 & 3.20 \\
\hline & (8.46-8.77) & $(1.78-2.66)$ & $(6.86-7.88)$ & $(3.03-3.39)$ \\
\hline \multirow[t]{2}{*}{12} & 14.10 & 3.75 & 13.45 & 5.13 \\
\hline & $(13.08-15.19)$ & $(3.12-4.50)$ & $(11.58-15.61)$ & $(4.93-5.35)$ \\
\hline \multirow[t]{2}{*}{13} & 7.47 & 3.69 & 5.13 & 4.85 \\
\hline & $(7.28-7.65)$ & $(2.90-4.70)$ & $(4.92-5.35)$ & $(4.56-5.16)$ \\
\hline \multirow[t]{2}{*}{14} & 11.09 & 2.41 & 14.57 & 4.37 \\
\hline & $(10.47-11.75)$ & $(1.54-3.78)$ & $(12.54-16.92)$ & $(4.22-4.52)$ \\
\hline \multirow[t]{2}{*}{15} & 9.13 & 2.03 & 15.30 & 4.86 \\
\hline & $(8.88-9.39)$ & $(1.50-2.75)$ & $(14.53-16.12)$ & $(4.74-4.99)$ \\
\hline \multirow[t]{2}{*}{ D } & 0.79 & 1.16 & 2.19 & 2.60 \\
\hline & $(0.41-1.55)$ & $(0.91-1.47)$ & $(1.76-2.73)$ & $(2.05-3.30)$ \\
\hline
\end{tabular}

aPreviously published data (14). PA: Protoapigenone; D: doxorubicin, n.d.: not determined; $\mathrm{n}=3$.

potent as compound $\mathbf{1}$ in the case of the Hep3B cell line. The overall tendency, however, suggests the importance of a free 7-OH group in order to have stronger activity when a longer aliphatic side-chain is present at C-1'.

Compounds (1-15), as well as protoapigenone, WYC0209 and their corresponding analogs (16-29) were also tested for their cytotoxic activity towards MDR mouse lymphoma cell line expressing the human ABCB1 pump, and against its parental cell line; results are shown in Table II.

The fact that none of the tested compounds exerted weaker activity on the MDR cell line than on the PAR cell line suggests that cancer cells cannot exhibit resistance to protoflavone derivatives by overexpressing the ABCB1 efflux pump. Moreover, for derivatives of 6-methylflavone, selective killing of the ABCB1 overexpressing MDR cancer cells takes place with a rather small ( 1.5 fold $)$, yet statistically significant, selectivity. Some selectivity against MDR cell lines overexpressing energy-consuming efflux pump systems could be due to a generally higher susceptibility of these cells whenever they encounter cytotoxic agents that are not ligands of the pumps. However, as from all tested compounds only the 6-methylflavone derivatives were found to be selective towards the MDR cell line, more specific reasons are to be suspected, in which presence of a 6-methyl group seems to be important. Interestingly, such a functional group (although connected to a non-aromatic A-ring) is also present in the unique flavone desmosdumotin $\mathrm{B}$, which was found to exert selective cytotoxic activity towards the ABCB1 expressing MDR cell line $\mathrm{KB}-\mathrm{VIN}$, with an $\mathrm{IC}_{50}$ value of $2.0 \mu \mathrm{g} / \mathrm{ml}$ in contrast to the $>40 \mu \mathrm{g} / \mathrm{ml}$ found towards the parental cell line (19).

In view of our results, 6-methylated protoflavone derivatives may serve as valuable leads toward the development of a potent and selective agent against MDR cancer cells overexpressing the $\mathrm{ABCB} 1$ transporter; design and synthesis of further analogs with the aim of exploring relevant structure activity relationships is currently in progress.

\section{Experimental}

Chemical characterization data for compounds 1-15 are presented below.

5-Hydroxy-2-(1-hydroxy-4-oxocyclohexa-2,5-dienyl)-7methoxy-4H-chromen-4-one (1)

Yield: $57 \% ;{ }^{1} \mathrm{H}$ NMR $\left(500 \mathrm{MHz}\right.$, acetone- $\left.d_{6}\right) \delta 12.6\left({ }^{1} \mathrm{H}, \mathrm{s}\right.$, OH-8), 7.03 (2H, d, J=10 Hz, H-2' and H-6'), $6.62(1 \mathrm{H}, \mathrm{s}$, $\mathrm{H}-3), 6.45(1 \mathrm{H}, \mathrm{s}, J=2.1 \mathrm{~Hz}, \mathrm{H}-8), 6.34(2 \mathrm{H}, \mathrm{d}, J=10.0 \mathrm{~Hz}$, H-3' and H-5'), 6.31 (d, $J=1.85 \mathrm{~Hz}, \mathrm{H}-6), 3.87$ (3H, s, OMe7); ESI-MS $(\mathrm{m} / \mathrm{z})$ : $301\left(\mathrm{M}^{+}+\mathrm{H}\right)$; ESI-MS-MS $(\mathrm{m} / \mathrm{z}, \%): 83.3$ (14), 223.3 (27), 256.0 (7), 284.1 (14), 301.2 (100).

5-Hydroxy-7-methoxy-2-(1-methoxy-4-oxocyclohexa-2,5dienyl)-4H-chromen-4-one (2)

Yield: $62 \%$; ${ }^{1} \mathrm{H}$ NMR (500 MHz, chloroform- $\left.d_{1}\right) \delta 12.43$ $(1 \mathrm{H}, \mathrm{s}, \mathrm{OH}-8), 6.77\left(2 \mathrm{H}, \mathrm{d}, J=10.0 \mathrm{~Hz}, \mathrm{H}-2\right.$ ' and $\left.\mathrm{H}-6^{\prime}\right), 6.61$ $(1 \mathrm{H}, \mathrm{s}, 1 \mathrm{H}, \mathrm{s}, \mathrm{H}-3), 6.56\left(2 \mathrm{H}, \mathrm{d}, J=10.0 \mathrm{~Hz}, \mathrm{H}-3\right.$ ' and $\left.\mathrm{H}-5^{\prime}\right)$, $6.32(1 \mathrm{H}, \mathrm{d}, J=1.8 \mathrm{~Hz}, \mathrm{H}-8), 6.25(1 \mathrm{H}, \mathrm{d}, J=1.8 \mathrm{~Hz}, \mathrm{H}-6)$, $3.82(3 \mathrm{H}, \mathrm{s}, \mathrm{OMe}-7), 3.39$ (3H, s, OMe"); ESI-MS $(\mathrm{m} / \mathrm{z})$ : $315\left(\mathrm{M}^{+}+\mathrm{H}\right)$; ESI-MS-MS $(\mathrm{m} / \mathrm{z}, \%): 243.3$ (2), 256.3 (16), $284.2(100), 315.2(47)$.

2-(1-Ethoxy-4-oxocyclohexa-2,5-dienyl)-5-hydroxy-7methoxy-4H-chromen-4-one (3)

Yield: $59.2 \%$; ${ }^{1} \mathrm{H}$ NMR (500 MHz, chloroform- $\left.d_{1}\right) \delta 12.53$ $(1 \mathrm{H}, \mathrm{s}, \mathrm{OH}-8), 6.79$ (2H, d, J=10.1 Hz, H-2' and H-6'), 6.67 $(1 \mathrm{H}, \mathrm{s}, \mathrm{H}-3), 6.52\left(2 \mathrm{H}, \mathrm{d}, J=10.1 \mathrm{~Hz}, \mathrm{H}-3^{\prime}\right.$ ' and $\left.\mathrm{H}-5^{\prime}\right), 6.32$ $(1 \mathrm{H}, \mathrm{d}, J=2.1 \mathrm{~Hz}, \mathrm{H}-8), 6.23(1 \mathrm{H}, \mathrm{d}, J=2.1 \mathrm{~Hz}, \mathrm{H}-6), 3.81$ $(3 \mathrm{H}, \mathrm{s}, \mathrm{OMe}), 3.57(2 \mathrm{H}, \mathrm{q}, J=6.9 \mathrm{~Hz}, \mathrm{H}-1$ ”), $1.27(3 \mathrm{H}, \mathrm{t}$, $J=6.5 \mathrm{~Hz}, \mathrm{H}-2 ")$; ESI-MS $(\mathrm{m} / \mathrm{z}, \%): 329.1\left(\mathrm{M}^{+}+\mathrm{H}\right)$; ESI-MS- 
Table II. Cytotoxic activity of protoapigenone, WYC0209 and compounds 1-29 towards the multidrug resistant cell line (MDR) and its parental cell line $(P A R)$.

\begin{tabular}{|c|c|c|c|c|c|c|c|}
\hline \multirow[b]{2}{*}{ Compound } & \multicolumn{2}{|c|}{$\mathrm{IC}_{50}(\mu \mathrm{M})$} & \multirow[b]{2}{*}{ Selectivitya } & \multirow[b]{2}{*}{ Compound } & \multicolumn{2}{|c|}{$\mathrm{IC}_{50}(\mu \mathrm{M})$} & \multirow[b]{2}{*}{ Selectivity } \\
\hline & PAR & MDR & & & PAR & MDR & \\
\hline 1 & $\begin{array}{c}1.72 \\
(1.57-1.90)\end{array}$ & $\begin{array}{c}1.51 \\
(1.28-1.78)\end{array}$ & n.s. & PA & $\begin{array}{c}0.76 \\
(0.66-0.88)\end{array}$ & $\begin{array}{c}0.79 \\
(0.71-0.88)\end{array}$ & n.s. \\
\hline 2 & $\begin{array}{c}0.55 \\
(0.45-0.67)\end{array}$ & $\begin{array}{c}0.57 \\
(0.50-0.65)\end{array}$ & n.s. & 16 & $\begin{array}{c}3.24 \\
(2.71-3.87)\end{array}$ & $\begin{array}{c}2.50 \\
(2.04-3.08)\end{array}$ & n.s. \\
\hline 3 & $\begin{array}{c}1.36 \\
(1.20-1.54)\end{array}$ & $\begin{array}{c}1.41 \\
(1.21-1.64)\end{array}$ & n.s. & 17 & $\begin{array}{c}3.72 \\
(3.11-4.46)\end{array}$ & $\begin{array}{c}3.43 \\
(2.83-4.16)\end{array}$ & n.s. \\
\hline 4 & $\begin{array}{c}1.47 \\
(1.26-1.73)\end{array}$ & $\begin{array}{c}1.63 \\
(1.41-1.87)\end{array}$ & n.s. & 18 & $\begin{array}{c}4.66 \\
(3.50-6.21)\end{array}$ & $\begin{array}{c}3.69 \\
(2.57-5.31)\end{array}$ & n.s. \\
\hline 5 & n.d. & n.d. & n.d. & 19 & $\begin{array}{c}7.07 \\
(4.89-10.23)\end{array}$ & $\begin{array}{c}8.16 \\
(3.41-19.55)\end{array}$ & n.s. \\
\hline 6 & $\begin{array}{c}3.95 \\
(3.17-4.93)\end{array}$ & $\begin{array}{c}3.98 \\
(3.58-4.42)\end{array}$ & n.s. & 20 & $\begin{array}{c}1.29 \\
(1.10-1.53)\end{array}$ & $\begin{array}{c}1.11 \\
(0.92-1.33)\end{array}$ & n.s. \\
\hline 7 & $\begin{array}{c}1.17 \\
(1.00-1.36)\end{array}$ & $\begin{array}{c}1.00 \\
(0.87-1.15)\end{array}$ & n.s. & 21 & $\begin{array}{c}1.27 \\
(1.08-1.49)\end{array}$ & $\begin{array}{c}1.20 \\
(1.09-1.33)\end{array}$ & n.s. \\
\hline 8 & $\begin{array}{c}1.18 \\
(0.96-1.45)\end{array}$ & $\begin{array}{c}0.97 \\
(0.83-1.12)\end{array}$ & n.s. & 22 & $\begin{array}{c}1.96 \\
(1.64-2.35)\end{array}$ & $\begin{array}{c}1.91 \\
(1.62-2.26)\end{array}$ & n.s. \\
\hline 9 & $\begin{array}{c}0.26 \\
(0.23-0.29)\end{array}$ & $\begin{array}{c}0.16 *(\mathrm{~T}) \\
(0.15-0.18)\end{array}$ & 1.63 & WYC0209 & $\begin{array}{c}0.15 \\
(0.14-0.16)\end{array}$ & $\begin{array}{c}0.13 \\
(0.11-0.14)\end{array}$ & n.s. \\
\hline 10 & $\begin{array}{c}1.85 \\
(1.64-2.07)\end{array}$ & $\begin{array}{c}1.35 * * \\
(1.15-1.59)\end{array}$ & 1.37 & 23 & $\begin{array}{c}0.95 \\
(0.81-1.13)\end{array}$ & $\begin{array}{c}0.82 \\
(0.70-0.95)\end{array}$ & n.s. \\
\hline 11 & $\begin{array}{c}2.38 \\
(2.08-2.73)\end{array}$ & $\begin{array}{c}1.75^{* *} \\
(1.50-2.05)\end{array}$ & 1.36 & 24 & $\begin{array}{c}1.46 \\
(1.22-1.75)\end{array}$ & $\begin{array}{c}1.34 \\
(1.16-1.55)\end{array}$ & n.s. \\
\hline 12 & $\begin{array}{c}4.61 \\
(3.83-5.56)\end{array}$ & $\begin{array}{c}2.94 * * * \\
(2.46-3.53)\end{array}$ & 1.57 & 25 & $\begin{array}{c}1.71 \\
(1.53-1.92)\end{array}$ & $\begin{array}{c}1.79 \\
(1.60-2.01)\end{array}$ & n.s. \\
\hline 13 & $\begin{array}{c}3.71 \\
(3.33-4.14)\end{array}$ & $\begin{array}{c}2.71 * * * \\
(2.29-3.21)\end{array}$ & 1.37 & 26 & $\sim 6.79$ & $\begin{array}{c}8.49 \\
(4.66-15.46)\end{array}$ & n.s. \\
\hline 14 & $\begin{array}{c}2.27 \\
(1.94-2.66)\end{array}$ & $\begin{array}{c}1.73 * * \\
(1.50-1.98)\end{array}$ & 1.31 & 27 & $\begin{array}{c}1.44 \\
(1.25-1.66)\end{array}$ & $\begin{array}{c}1.26 \\
(1.12-1.43)\end{array}$ & n.s. \\
\hline 15 & $\begin{array}{c}1.45 \\
(1.29-1.63)\end{array}$ & $\begin{array}{c}0.98^{*} \\
(0.88-1.08)\end{array}$ & 1.48 & 28 & $\begin{array}{c}0.82 \\
(0.70-0.96)\end{array}$ & $\begin{array}{c}0.74 \\
(0.63-0.87)\end{array}$ & n.s. \\
\hline D & $\begin{array}{c}0.61 \\
(0.51-0.74)\end{array}$ & $\begin{array}{c}8.60 * * * \\
(7.80-9.47)\end{array}$ & 0.071 & 29 & $\begin{array}{c}0.76 \\
(0.67-0.85)\end{array}$ & $\begin{array}{c}0.81 \\
(0.65-1.01)\end{array}$ & n.s. \\
\hline
\end{tabular}

aSelectivity was calculated for those compounds which showed significant differences in the $\mathrm{IC}_{50}$ values towards the two cells lines. $95 \%$ Confidence intervals are presented for each curve fitting result, : ambiguous fitting, no confidence interval available. Selectivity=IC ${ }_{50}{ }^{(\mathrm{PAR}) / \mathrm{IC}}{ }_{50}{ }^{(\mathrm{MDR})}$. Statistical comparisons were made between values in columns PAR and MDR. ${ }^{*} p<0.05, * * p<0.01$ and $* * * p<0.001$ by one-way ANOVA followed by Bonferroni's multiple comparison test; ${ }^{*}(\mathrm{~T}): p<0.05$ by unpaired $t$-test, variances are not significantly different; $\mathrm{D}$ : doxorubicin, n.d.: not determined, n.s.: no selectivity, $\mathrm{n}=3$.

MS $(m / z, \%): 54.1(3), 86.3(4), 243.2(4), 256.2(14), 284$ (100), 301.1 (4), 329.3 (52).

5-Hydroxy-7-methoxy-2-(4-oxo-1-propoxycyclohexa-2,5dienyl)-4H-chromen-4-one (4)

Yield: $68.8 \%$; ${ }^{1} \mathrm{H}$ NMR $\left(500 \mathrm{MHz}\right.$, chloroform- $\left.d_{1}\right) \delta 12.41$ (1H, s, OH-8) 6.76 (2H, d, J=10.0 Hz, H-2' and H-6'), 6.66 (1H, s, H-3), 6.52 (2H, d, $J=10.0 \mathrm{~Hz}, \mathrm{H}-3$ ' and H-5'), 6.32 $(1 \mathrm{H}, \mathrm{d}, J=1.6 \mathrm{~Hz}, \mathrm{H}-8), 6.23(1 \mathrm{H}, \mathrm{d}, J=1.4 \mathrm{~Hz}, \mathrm{H}-6), 3.81$ (3H,s, OMe), 3.45 (2H, t , J=6.4 Hz, H-1”), 1.65 (3H, m, H2”), 0.96 (3H, t, J=7.4 Hz, H-3”); ESI-MS ( $\mathrm{m} / \mathrm{z}, \%): 343.3$ $\left(\mathrm{M}^{+}+\mathrm{H}\right)$; ESI-MS-MS (m/z, \%): 54.4 (6), 86.5(10), $243.2(5)$, 257.1 (12), 273.1 (2), 284.3 (100), 301.3 (2), 343.2 (40)
5-Hydroxy-2-(1-isopropoxy-4-oxocyclohexa-2,5-dienyl)-7methoxy-4H-chromen-4-one (5)

Yield: 49.4\%; ${ }^{1} \mathrm{H}$ NMR (500 MHz, chloroform- $d_{1}$ ) $\delta 12.42$ (1H, s, H-8), 6.80 (2H, d, J=9.9 Hz, H-2' and H-6'), 6.67 (1H, s, H-3), 6.49 (2H, d, $J=10.0 \mathrm{~Hz}, \mathrm{H}-3$ ' and H-5'), 6.30 (1H, d, J=1.8 Hz, H-8), $6.21(1 \mathrm{H}, \mathrm{d}, J=2.0 \mathrm{~Hz}, \mathrm{H}-6), 3.83$ (1H, dd, J=12.2, $6.1 \mathrm{~Hz}, \mathrm{H}-1$ "), 3.80 (3H, s, OMe), 1.21 $(6 \mathrm{H}, \mathrm{d}, J=6.1 \mathrm{~Hz}, \mathrm{H}-2$ " and H-3"); ESI-MS $(\mathrm{m} / \mathrm{z}): 343.0$ $\left(\mathrm{M}^{+}+\mathrm{H}\right)$; ESI-MS-MS (m/z, \%): 54.3 (6), 86.3(9), 242.5 (4), 256 (13), 272.9 (6), 284.3 (100), 301.3 (8), 343.1 (68).

2-(1-Ethoxy-4-oxocyclohexa-2,5-dienyl)-5-hydroxy-7methoxy-4H-chromen-4-one (6) 
Yield: $33 \%$; ${ }^{1} \mathrm{H}$ NMR (500 MHz, chloroform- $d_{1}$ ) $\delta 12.44$ $(1 \mathrm{H}, \mathrm{s}, \mathrm{H}-8), 6.77\left(2 \mathrm{H}, \mathrm{d}, J=10 \mathrm{~Hz}, \mathrm{H}-2^{\prime}\right.$ and H-6'), 6.68 $(1 \mathrm{H}, \mathrm{s}, \mathrm{H}-3), 6.54(2 \mathrm{H}, \mathrm{d}, J=10 \mathrm{~Hz}, \mathrm{H}-3$ ' and H-5'), $6.33(1 \mathrm{H}$, d, $J=1.85 \mathrm{~Hz}, \mathrm{H}-8), 6.25(1 \mathrm{H}, \mathrm{d}, J=2.0 \mathrm{~Hz}, \mathrm{H}-6) .3 .83(3 \mathrm{H}$, s, OMe), 3.51 (2H, s, H-1"), 1.62 (2H, m , H-2"), 1.43 (2H, m, H-3"), 0.92 (3H, t, J=7.4 Hz, H-4"); ESI-MS (m/z): 357 $\left(\mathrm{M}^{+}+\mathrm{H}\right)$; ESI-MS-MS ( $\left.\mathrm{m} / \mathrm{z}, \%\right): 100.5$ (1), 243.1 (5), 257.2 (14),273.2 (4), 284.2 (100), 301.1 (8), 329.3 (1), 357.1 (72). 2-(1-(Allyloxy)-4-oxocyclohexa-2,5-dienyl)-5-hydroxy-7methoxy-4H-chromen-4-one (7)

Yield: $43.6 \%$; ${ }^{1} \mathrm{H}$ NMR (500 MHz, chloroform- $\left.d_{1}\right) \delta 12.39$ (1H, s, H-8), 6.79 (2H, d, J=10.1 Hz, H-2' and H-6'), 6.67 $(1 \mathrm{H}, \mathrm{s}, \mathrm{H}-3), 6.53\left(2 \mathrm{H}, \mathrm{d}, J=10.1 \mathrm{~Hz}, \mathrm{H}-3^{\prime}\right.$ ' and H-5'), 6.31 $(1 \mathrm{H}, \mathrm{d}, J=2.1 \mathrm{~Hz}, \mathrm{H}-8), 6.23(1 \mathrm{H}, \mathrm{d}, J=1.8 \mathrm{~Hz}, \mathrm{H}-6), 5.91$ $(1 \mathrm{H}, \mathrm{m}, J=5.2 \mathrm{~Hz}, \mathrm{H}-2$ "), 5.33 (1H, d, $J=17.2 \mathrm{~Hz}, \mathrm{H}-3$ ", cisz), $5.22(1 \mathrm{H}, \mathrm{d}, J=10.4 \mathrm{~Hz}, \mathrm{H}-3$ " , trans $), 4.04(1 \mathrm{H}, \mathrm{m}, \mathrm{H}-$ 1"), 3.81 ( $3 \mathrm{H}, \mathrm{s}, \mathrm{OMe})$; ESI-MS $(\mathrm{m} / \mathrm{z}): 341.3\left(\mathrm{M}^{+}+\mathrm{H}\right)$; ESIMS-MS ( $\mathrm{m} / \mathrm{z}, \%): 243.2$ (6), 257.2 (16), 272.3 (2), 284.1 (100), 300.1 (2), 341.3 (42).

5-Hydroxy-7-methoxy-2-(4-oxo-1-(prop-2-ynyloxy) cyclohexa-2,5-dienyl)-4H-chromen-4-one (8)

Yield: $37.2 \%$; ${ }^{1} \mathrm{H}$ NMR (500 MHz, chloroform- $\left.d_{1}\right) \delta 12.38$ $(1 \mathrm{H}, \mathrm{s}, \mathrm{H}-8), 6.85\left(2 \mathrm{H}, \mathrm{d}, J=9.9 \mathrm{~Hz}, \mathrm{H}-2^{\prime}\right.$ ' and H-6'), 6.66 $(1 \mathrm{H}, \mathrm{s}, \mathrm{H}-3), 6.55\left(2 \mathrm{H}, \mathrm{d}, J=9.9 \mathrm{~Hz}, \mathrm{H}-3^{\prime}\right.$ and $\mathrm{H}-5$ '), 6.32 $(1 \mathrm{H}, \mathrm{d}, J=1.8 \mathrm{~Hz}, \mathrm{H}-8), 6.24(1 \mathrm{H}, \mathrm{d}, J=1.8 \mathrm{~Hz}, \mathrm{H}-6), 4.21$ $(2 \mathrm{H}, \mathrm{d}, J=2.1 \mathrm{~Hz}, \mathrm{H} 1$ ”), $3.81(3 \mathrm{H}, \mathrm{s}, \mathrm{OMe}), 2.52(1 \mathrm{H}, \mathrm{t}$, $J=2.4 \mathrm{~Hz}, \mathrm{H}-3 ")$; ESI-MS $(\mathrm{m} / \mathrm{z}, \%): 339.3\left(\mathrm{M}^{+}+\mathrm{H}\right.$,); ESIMS-MS ( $/ \mathrm{z}, \%)$ : 49.2 (4), 243.3 (6), 256.2 (18), 271.2 (3), 284.3 (100), 300.3 (2), 339.2 (42).

2-(1-Hydroxy-4-oxocyclohexa-2,5-dienyl)-6-methyl- $4 H$ chromen-4-one (9)

Yield: $48.2 \%$; ${ }^{1} \mathrm{H}$ NMR (500 MHz, chloroform- $\left.d_{1}\right) \delta 7.93$ $(1 \mathrm{H}, \mathrm{s}, \mathrm{H}-7), 7.47$ (1H, d, $J=8.5 \mathrm{~Hz}, \mathrm{H}-8), 7.27$ (1H, d, $J=8.6$ $\mathrm{Hz}, \mathrm{H}-5), 6.94(2 \mathrm{H}, \mathrm{d}, J=9.5 \mathrm{~Hz}, \mathrm{H}-2$ ' and H-6'), $6.84(1 \mathrm{H}$, s, H-3), 6.41 (2H, d, J=9.5 Hz, H-3' and H-5'), 2.43 (3H s, CH3-6); ESI-MS $(\mathrm{m} / \mathrm{z}): 269.1\left(\mathrm{M}^{+}+\mathrm{H},\right)$; ESI-MS-MS $(\mathrm{m} / \mathrm{z}$, \%): 83.5 (1), 92.4 (5), 107.6 (6), 120.7 (11), 134 (30), 147 (14), 224.2 (1), 241.1 (16), 252 (16), 269.2 (100).

2-(1-Methoxy-4-oxocyclohexa-2,5-dienyl)-6-methyl-4Hchromen-4-one (10)

Yield: 59.7\%; ${ }^{1} \mathrm{H}$ NMR (500 MHz, methanol- $\left.d_{4}\right) \delta 7.87(1 \mathrm{H}$, s, H-7), $7.57(1 \mathrm{H}, \mathrm{d}, J=8.5 \mathrm{~Hz}, \mathrm{H}-8), 7.33(1 \mathrm{H}, \mathrm{d}, J=8.6 \mathrm{~Hz}$, H-5), 6.97 (2H, d, J=9.9 Hz, H-2' and H-6'), $6.71(1 \mathrm{H}, \mathrm{s}, \mathrm{H}-$ 3), $6.57\left(2 \mathrm{H}, \mathrm{d}, J=9.9 \mathrm{~Hz}, \mathrm{H}-3^{\prime}\right.$ and H-5'), 3.44 (3H s, H1”), 2.43 (3H s, CH3-6); ESI-MS ( $\mathrm{m} / \mathrm{z}): 283.2\left(\mathrm{M}^{+}+\mathrm{H}\right.$ ); ESIMS-MS (m/z, \%): 133.8 (1), 146.9 (3), 224.2 (20), 252.2 (100), 283.1 (25).

2-(1-Ethoxy-4-oxocyclohexa-2,5-dienyl)-6-methyl-4Hchromen-4-one (11)

Yield: $20 \% ;{ }^{1} \mathrm{H}$ NMR $\left(500 \mathrm{MHz}\right.$, methanol- $\left.\left.d_{4}\right)\right) \delta 7.88(1 \mathrm{H}$, s, H-7), $7.59(1 \mathrm{H}, \mathrm{d}, J=8.6 \mathrm{~Hz}, \mathrm{H}-8), 7.33(1 \mathrm{H}, \mathrm{d}, J=8.6 \mathrm{~Hz}$, H-5), $7.00(2 \mathrm{H}, \mathrm{d}, J=10.1 \mathrm{~Hz}, \mathrm{H}-2$ ' and $\mathrm{H}-6$ ') $6.75(1 \mathrm{H}, \mathrm{s}$, H-3), $6.54(2 \mathrm{H}, \mathrm{d}, J=10,0 \mathrm{~Hz}, \mathrm{H}-3$ ' and H-5'), 3.64 (2H q,
$J=6.9 \mathrm{~Hz}, \mathrm{H}-1$ "), 2.44 (3H s, CH3-6), 1.30 (3H, t, $J=7.0 \mathrm{~Hz}$, H-2"); ESI-MS $(\mathrm{m} / \mathrm{z}): 296.8\left(\mathrm{M}^{+}+\mathrm{H},\right)$; ESI-MS-MS $(\mathrm{m} / \mathrm{z}$, \%): 224.2 (18), 241 (2), 252.1 (100), 269.2 (3), 297.3 (28). 6-Methyl-2-(4-oxo-1-propoxycyclohexa-2,5-dienyl)- $4 H$ chromen-4-one (12)

Yield: $36 \% ;{ }^{1} \mathrm{H}$ NMR $\left(500 \mathrm{MHz}\right.$, methanol- $\left.\left.d_{4}\right)\right) \delta 7.89(1 \mathrm{H}$, s, H-7), $7.59(1 \mathrm{H}, \mathrm{d}, J=8.5 \mathrm{~Hz}, \mathrm{H}-8), 7.34(1 \mathrm{H}, \mathrm{d}, J=8.6 \mathrm{~Hz}$, H-5), $7.00(2 \mathrm{H}, \mathrm{d}, J=10.1 \mathrm{~Hz}, \mathrm{H}-2$ ' and H-6'), $6.75(1 \mathrm{H}, \mathrm{s}$, $\mathrm{H}-3), 6.54(2 \mathrm{H}, \mathrm{d}, J=10,0 \mathrm{~Hz}, \mathrm{H}-3$ ' and $\mathrm{H}-5$ '), $3.54(2 \mathrm{H} \mathrm{q}$, $J=6.3 \mathrm{~Hz}, \mathrm{H}-1$ ”), 2.45 (3H s, CH3-6), 1.75 (sextet, $J=6.8 \mathrm{~Hz}$ H-2"), 1.01 (3H, t, $J=7.3 \mathrm{~Hz}, \mathrm{H}-3 ")$; ESI-MS $(\mathrm{m} / \mathrm{z}): 311.3$ $\left(\mathrm{M}^{+}+\mathrm{H},\right)$; ESI-MS-MS $(\mathrm{m} / \mathrm{z}, \%): 54.4(12), 86.6(11), 224.1$ (20), 241.1 (7), 252.3 (100), 268.8 (3), 311 (28).

2-(1-Butoxy-4-oxocyclohexa-2,5-dienyl)-6-methyl-4Hchromen-4-one (13)

Yield: $35 \% ;{ }^{1} \mathrm{H}$ NMR $\left(500 \mathrm{MHz}\right.$, methanol- $\left.\left.d_{4}\right)\right) \delta 7.89(1 \mathrm{H}$, s, H-7), $7.59(1 \mathrm{H}, \mathrm{d}, J=8.5 \mathrm{~Hz}, \mathrm{H}-8), 7.34(1 \mathrm{H}, \mathrm{d}, J=8.6 \mathrm{~Hz}$, H-5), 6.99 (2H, d, $J=10.0 \mathrm{~Hz}, \mathrm{H}-2$ ' and H-6'), $6.75(1 \mathrm{H}, \mathrm{s}$, $\mathrm{H}-3), 6.55(2 \mathrm{H}, \mathrm{d}, J=10,0 \mathrm{~Hz}, \mathrm{H}-3$ ' and $\mathrm{H}-5$ '), $3.59(2 \mathrm{H}, \mathrm{t}$, $J=6.2 \mathrm{~Hz}, \mathrm{H}-1$ "), 2.45 (3H s, CH3-6), 1.66 (2H, m , H-2"), 1.48 (2H, m, H-3"), 0.97 (3H, t, J=7.4 Hz, H-4"); ESI-MS $(\mathrm{m} / \mathrm{z}): 325.3\left(\mathrm{M}^{+}+\mathrm{H},\right)$; ESI-MS-MS $(\mathrm{m} / \mathrm{z}, \%): 225.2(18)$, 241.2 (7), 252.2 (100), 269 (9), 325 (4).

2-(1-(Allyloxy)-4-oxocyclohexa-2,5-dienyl)-6-methyl- $4 H$ chromen-4-one (14)

Yield: $73 \%$; ${ }^{1} \mathrm{H}$ NMR $\left(500 \mathrm{MHz}\right.$, methanol- $\left.\left.d_{4}\right)\right) \delta 7.77(1 \mathrm{H}$, s, H-7), $7.47(1 \mathrm{H}, \mathrm{d}, J=8.5 \mathrm{~Hz}, \mathrm{H}-8), 7.23(1 \mathrm{H}, \mathrm{d}, J=8.6 \mathrm{~Hz}$, H-5), $6.91(2 \mathrm{H}, \mathrm{d}, J=10.0 \mathrm{~Hz}, \mathrm{H}-2$ ' and H-6'), $6.66(1 \mathrm{H}, \mathrm{s}$, $\mathrm{H}-3), 6.45\left(2 \mathrm{H}, \mathrm{d}, J=9,9 \mathrm{~Hz}, \mathrm{H}-3^{\prime}\right.$ and $\left.\mathrm{H}^{-} 5^{\prime}\right), 5.90(1 \mathrm{H}, \mathrm{m}$, $J=5.3 \mathrm{~Hz}, \mathrm{H}-2 "), 5.27$ (1H, d, $J=17.2 \mathrm{~Hz}, \mathrm{H}-3$ ", cisz), 5.12 $(1 \mathrm{H}, \mathrm{d}, J=10.4 \mathrm{~Hz}, \mathrm{H}-3$ ", trans) $4.02(2 \mathrm{H}, \mathrm{d}, J=5.2 \mathrm{~Hz}, \mathrm{H}-$ 1"), 2.33 (3H s, CH3-6); ESI-MS ( $/ \mathrm{z} / \mathrm{z}): 309.1\left(\mathrm{M}^{+}+\mathrm{H}\right.$,); ESIMS-MS ( $/ \mathrm{z}, \%): 224.1$ (14), 240 (18), 252 (7), 268.2 (19), 281.1 (21), 309.1 (100).

6-Methyl-2-(4-oxo-1-(prop-2-ynyloxy)cyclohexa-2,5-dienyl)$4 H$-chromen-4-one (15)

Yield: $63 \%$; ${ }^{1} \mathrm{H}$ NMR $\left(500 \mathrm{MHz}\right.$, methanol- $\left.\left.d_{4}\right)\right) \delta 7.77(1 \mathrm{H}, \mathrm{s}$, H-7), $7.48(1 \mathrm{H}, \mathrm{d}, J=8.4 \mathrm{~Hz}, \mathrm{H}-8), 7.23(1 \mathrm{H}, \mathrm{d}, J=8.6 \mathrm{~Hz}, \mathrm{H}-$ 5), $6.95\left(2 \mathrm{H}, \mathrm{d}, J=10.1 \mathrm{~Hz}, \mathrm{H}-2\right.$ ' and $\left.\mathrm{H}-6^{\prime}\right), 6.62(1 \mathrm{H}, \mathrm{s}, \mathrm{H}-$ 3), $6.46\left(2 \mathrm{H}, \mathrm{d}, J=10,1 \mathrm{~Hz}, \mathrm{H}-3 \text { ' and } \mathrm{H}^{-}\right)^{\prime}, 4.21(2 \mathrm{H}, \mathrm{d}, J=2.2$ $\mathrm{Hz}, \mathrm{H}-1$ "), 2.89 (t, J=2.3 Hz, 2H, H-3"), 2.34 (3H s, CH3-6); ESI-MS $(\mathrm{m} / \mathrm{z}): 306.9\left(\mathrm{M}^{+}+\mathrm{H},\right)$; ESI-MS-MS $(\mathrm{m} / \mathrm{z}, \%): 49.2$ (1), 224.2 (23), 252.3 (100), 268 (2), 279 (1), 307.2 (37).

\section{Acknowledgements}

This work was conducted within the framework of COST Actions CM0804 and BM0701. The research was supported by grant from the National Science Council, Executive Yuan, Taiwan (NSC 1012325-B-039-004), the National SunYat-Sen University-Kaohsiung Medical University Joint Research Center (Y.-C. Wu), the Hungarian Research Fund (OTKA K72771), the Szeged Foundation for Cancer Research and the New Hungary Development Plan (TÁMOP 4.2.1/B-09/1/KONV-2010-0005). 


\section{References}

1 Le Marchand L: Cancer preventive effects of flavonoids-a review. Biomed Pharmacother 56: 296-301, 2002.

2 Kandaswami C, Lee LT, Lee PP, Hwang JJ, Ke FC, Huang YT and Lee MT: The antitumor activities of flavonoids. In Vivo 19: 895-909, 2005.

$3 \mathrm{Li} \mathrm{Y,} \mathrm{Fang} \mathrm{H,} \mathrm{Xu} \mathrm{W:} \mathrm{Recent} \mathrm{advance} \mathrm{in} \mathrm{the} \mathrm{research} \mathrm{of}$ flavonoids as anticancer agents. Mini Rev Med Chem 7: 663678, 2007.

4 Kale A, Gawande S and Kotwal S: Cancer phytotherapeutics: role for flavonoids at the cellular level. Phytother Res 22: 567577, 2008

5 Lin AS, Chang FR, Wu CC, Liaw CC and Wu YC: New Cytotoxic Flavonoids from Thelypteris torresiana. Planta Med 71: 867-870, 2005.

6 Chang HL, Su JH, Yeh YT, Lee YC, Chen HM, Wu YC and Yuan SS: Protoapigenone, a novel flavonoid, inhibits ovarian cancer cell growth in vitro and in vivo. Cancer Lett 267: 85-95, 2008.

7 Chang HL, Wu YC, Su JH, Yeh YT and Yuan SSF: Protoapigenone, a novel flavonoid, induces apoptosis in human prostate cancer cells through activation of p38 mitogen-activated protein kinase and c-Jun $\mathrm{NH}_{2}$-terminal kinase 1/2. J Pharmacol Exp Ther 325: 841-849, 2008.

8 Chiu CC, Chang HW, Chuang DW, Chang FR, Chang YC, Cheng YS, Tsai MT, Chen WY, Lee SS, Wang CK, Chen JY, Wang HM, Chen CC, Liu and Wu YC: Fern plant-derived protoapigenone leads to DNA damage, apoptosis, and $\mathrm{G}(2) / \mathrm{m}$ arrest in lung cancer cell line H1299. DNA Cell Biol 28: 501-506, 2009.

9 Chen WY, Hsieh YA, Tsai CI, Kang YF, Chang FR, Wu YC and $\mathrm{Wu} \mathrm{CC}$ : Protoapigenone, a natural derivative of apigenin, induces mitogen-activated protein kinase-dependent apoptosis in human breast cancer cells associated with induction of oxidative stress and inhibition of glutathione S-transferase $\pi$. Invest New Drugs 29(6): 1347-1359, 2010.

10 Wang HC, Lee AY, Chou WC, Wu CC, Tseng CN, Liu KY, Lin WL, Chang FR, Chuang DW, Hunyadi A and Wu YC: Inhibition of ATR-dependent signaling by protoapigenone and its derivative sensitize cancer cells to interstrand cross-link-generating agents in vitro and in vivo. Mol Cancer Ther, 2012. doi: 10.1158/15357163.MCT-11-0921.
11 Cimprich KA and Cortez D: ATR: an essential regulator of genome integrity. Nat Rev Mol Cell Biol 9: 616-627, 2008.

12 Tung CP, Chang FR, Wu YC, Chuang DW, Hunyadi A and Liu ST: Inhibition of the Epstein-Barr virus lytic cycle by protoapigenone. J Gen Virol 92: 1760-1768, 2011.

13 Lin AS, Nakagawa-Goto K, Chang FR, Yu D, Morris-Natschke SL, Wu CC, Chen SL, Wu YC and Lee KH: First total synthesis of protoapigenone and its analogues as potent cytotoxic agents. J Med Chem 50: 3921-3927, 2007.

14 Hunyadi A, Chuang DW, Danko B, Chiang MY, Lee CL, Wang $\mathrm{HC}$, Wu CC, Chang FR and Wu YC: Direct semi-synthesis of the anticancer lead-drug protoapigenone from apigenin, and synthesis of further new cytotoxic protoflavone derivatives. PLoS ONE 6(8): e23922, 2011.

15 Sharom FJ: ABC multidrug transporters: Structure, function and role in chemoresistance. Pharmacogenomics 9(1): 105-127, 2008.

16 Pastan I, Gottesman MM, Ueda K, Lovelace E, Rutherford AV and Willingham MC: A retrovirus carrying an MDR1 cDNA confers multidrug resistance and polarized expression of Pglycoprotein in MDCK cells. Proc Natl Acad Sci USA 85: 44864490, 1988.

17 Mosmann T: Rapid colorimetric assay for cellular growth and survival: Application to proliferation and cytotoxicity assays. J Immunol Methods 65: 55-63, 1983.

18 Bansal T, Jaggi M, Khar RK and Talegaonkar S: Emerging significance of flavonoids as P-glycoprotein inhibitors in cancer chemotherapy. J Pharm Pharmaceut Sci 12(1): 46-78, 2009.

19 Nakagawa-Goto K, Bastow KF, Wu JH, Tokuda H and Lee KH: Total synthesis and bioactivity of unique flavone desmosdumotin B and its analogs. Bioorg Med Chem Lett 15(12): 3016-3019, 2005 .
Received May 6, 2012

Revised June 5, 2012

Accepted June 6, 2012 


\title{
Protoapigenone derivatives: Albumin binding properties and effects on HepG2 cells
}

\author{
Miklós Poór ${ }^{\mathrm{a}}$, Yin Li ${ }^{\mathrm{b}, \mathrm{c}}$, Sándor Kunsági-Máté ${ }^{\mathrm{b}, \mathrm{c}}, Z$ Zsófia Varga ${ }^{\mathrm{a}}$, Attila Hunyadi ${ }^{\mathrm{d}}$, Balázs Dankó ${ }^{\mathrm{d}}$, \\ Fang-Rong Chang e,f,g , Yang-Chang Wu ${ }^{\text {h,i,j }}$, Tamás Kőszegi ${ }^{\mathrm{a}, *}$ \\ ${ }^{a}$ Institute of Laboratory Medicine, University of Pécs, Pécs H-7624, Hungary \\ ${ }^{\mathrm{b}}$ Department of General and Physical Chemistry, University of Pécs, Pécs H-7624, Hungary \\ 'János Szentágothai Research Center, Pécs H-7624, Hungary \\ ${ }^{\mathrm{d}}$ Institute of Pharmacognosy, University of Szeged, Szeged H-6720, Hungary \\ ${ }^{\mathrm{e}}$ Graduate Institute of Natural Products, Kaohsiung Medical University, Kaohsiung, Taiwan, ROC \\ ${ }^{\mathrm{f}}$ Cancer Center, Kaohsiung Medical University Hospital, 807 Kaohsiung, Shih-Chuan 1st Rd. 100, Taiwan, ROC \\ ${ }^{\mathrm{g}}$ RED Center of Chinese Herbal Medicines E New Drugs, College of Pharmacy, Kaohsiung Medical University, 807 Kaohsiung, Shih-Chuan 1st Rd. 100, Taiwan, ROC \\ ${ }^{\mathrm{h}}$ School of Pharmacy, College of Pharmacy, China Medical University, 404 Taichung, Hsueh-Shih Rd. 91, Taiwan, ROC \\ ${ }^{\mathrm{i}}$ Natural Medicinal Products Research Center, China Medical University Hospital, 404 Taichung, Hsueh-Shih Rd. 91, Taiwan, ROC \\ ${ }^{j}$ Center for Molecular Medicine, China Medical University Hospital, 404 Taichung, Hsueh-Shih Rd. 91, Taiwan, ROC
}

\section{A R T I C L E I N F O}

\section{Article history:}

Received 17 December 2012

Received in revised form 28 March 2013

Accepted 7 April 2013

Available online 13 April 2013

\section{Keywords:}

Flavonoids

Protoapigenone derivatives

Anticancer effect

Albumin binding

Cell viability

\begin{abstract}
A B S T R A C T
Protoapigenone (Pa) is a flavone aglycone with a p-quinol structure in its B-ring. It was first discovered in Thelypteris torresiana, a native fern in Taiwan. Recent studies highlighted that protoapigenone and some of its derivatives show very potent anticancer activity against several types of tumors, using both in vitro and in vivo models. Despite the growing body of evidence on the selective anticancer potential of protoapigenone and its derivatives, no data are available on their pharmacokinetical properties. In our present research, albumin binding properties of $\mathrm{Pa}$ and seven different $\mathrm{1}^{\prime}$-O-alkyl protoapigenone derivatives were analyzed as well as their biochemical effects on HepG2 tumor cell line in comparison with the flavone apigenin. Our results are in good accordance with the data of previous investigations of $1^{\prime}-O$-alkylated derivatives of protoapigenone (with the exception of isopropyl and allyl derivatives) showing similar or higher antitumor effects than Pa. Furthermore structural changes in Pa cause a very remarkable influence on plasma albumin binding affinity of the derivatives. Our investigation proves that parallel with changes of lipophilic character and extent of plasma protein binding properties of Pa derivatives a consequent alteration occurs in their pharmacokinetic behavior without losing the pharmacodynamic effect. Based on our study a better understanding of the structural and biochemical behavior of different chemically modified flavonoid derivatives could be achieved making further design of in vivo experiments feasible.
\end{abstract}

(c) 2013 Elsevier B.V. All rights reserved.

\section{Introduction}

Flavonoids are well-known natural polyphenols typically with significant free-radical scavenging and antioxidant properties [1]. In addition to the widespread knowledge on the molecular actions of flavonoids, recent publications have revealed the ability of these compounds to act via novel pathways influencing several biochemical systems: flavonoids have impact on different enzymes and transporters $[1,2]$, they can modulate numerous cell signaling pathways $[3,4]$ and several members of this molecule family show very strong albumin binding capacity [5,6]. Protoapigenone (Fig. 1), a less common flavone aglycone with a non-aromatic, p-quinol structure in its B-ring, was first discovered in Thelypteris torresiana,

* Corresponding author. Address: Institute of Laboratory Medicine, University of Pécs, Pécs H-7624, Ifjúság u. 13, Hungary. Tel.: +36 72536 120; fax: +36 72536121.

E-mail address: koszegit@freemail.hu (T. Köszegi). a native fern in Taiwan [7]. Recent studies suggest that protoapigenone and some of its derivatives show very promising selective anticancer activity against several types of tumors, both in vitro and in vivo [8-16]. The mechanism of action seems to be complex: induction of oxidative stress, inhibition of glutathione S-transferase, $\mathrm{S}$ and $\mathrm{G} 2 / \mathrm{M}$ phase cell cycle arrest, and induction of apoptotic cell death were also described [8-10]. Furthermore, selective toxicity against a multidrug resistant (MDR) cancer cell line [11] and antiviral activity against Ebstein-Barr virus (EBV) [12] was published. During the last 2 years the biochemical effectiveness of two further, synthetic protoapigenone analogs RY10-4 and WYC0209 has been also verified [13-15]. Probably the most interesting, recent finding concerning the mode of action of protoapigenone and WYC0209 is that they can inhibit ataxia telangiectasiamutated and Rad3-related (ATR) signaling both in vitro and in vivo [16]. As this pathway is known to play a crucial role in DNA damage response, these compounds are valuable leads for 
<smiles>O=c1cc(-c2ccc(O)cc2)oc2cc(O)cc(O)c12</smiles><smiles></smiles>

\begin{tabular}{|l|l|}
\hline \multicolumn{1}{|c|}{ Substance } & \multicolumn{1}{c|}{$\mathbf{R}$} \\
\hline Protoapigenone (Pa) & $-\mathrm{H}$ \\
\hline Protoapigenone 1'-O-methylether (Pa-Me) & $-\mathrm{CH}_{3}$ \\
\hline Protoapigenone 1'-O-ethylether (Pa-Et) & $-\mathrm{CH}_{2}-\mathrm{CH}_{3}$ \\
\hline Protoapigenone 1'-O-propylether (Pa-Pro) & $-\left(\mathrm{CH}_{2}\right)_{2}-\mathrm{CH}_{3}$ \\
\hline Protoapigenone 1'-O-isopropylether (Pa-iPro) & $-\mathrm{CH}^{-\mathrm{CH}_{3}}$ \\
& $\dot{\mathrm{C}} \mathrm{H}_{3}$ \\
\hline Protoapigenone 1'-O-butylether (Pa-But) & $-\left(\mathrm{CH}_{2}\right)_{3}-\mathrm{CH}_{3}$ \\
\hline Protoapigenone 1'-O-allylether (Pa-All) & $-\mathrm{CH}_{2}-\mathrm{CH}=\mathrm{CH}_{2}$ \\
\hline Protoapigenone 1'-O-propargylether (Pa-PIII) & $-\mathrm{CH}_{2}-\mathrm{C} \equiv \mathrm{CH}$ \\
\hline
\end{tabular}

Fig. 1. Chemical structure of apigenin, protoapigenone and its $1^{\prime}-O$-alkyl derivatives.

development of a combination therapy to be used with common DNA damaging chemotherapeutics, such as for example cisplatin [16]. Despite the growing body of evidence on the anticancer potential of protoapigenone and its derivatives, no data are available on their pharmacokinetic properties.

In the present study our main goal was the investigation of structure related biological differences between chemically modified Pa-derivatives. Therefore albumin binding properties of protoapigenone and seven different $1^{\prime}$-O-alkyl protoapigenone derivatives (Fig. 1) were analyzed as well as their antitumor effects on HepG2 tumor cell line in comparison with those of the 4'-hydroxyflavone apigenin (Fig. 1) using fluorescence and luminescence spectroscopy. Plasma albumin binding properties of biologically active molecules is a very important parameter because it plays a key role in the pharmacokinetic behavior of drugs and xenobiotics. Furthermore, data on the albumin binding characteristics of protoapigenone derivatives provide additional information on how albumin binding could be influenced by the chemical modification of native flavonoid aglycones. In order to investigate the cellular impacts of Pa-derivatives changes in cell cycle distribution and effects on cell viability (intracellular esterase activity, ATP and total protein levels) were examined.

\section{Materials and methods}

\subsection{Reagents}

All reagents and solvents were of analytical grade. Protoapigenone $(\mathrm{Pa})$ and its $1^{\prime}$-O-alkyl derivatives: protoapigenone $1^{\prime}$-O-methylether (Pa-Me), protoapigenone 1'-O-ethylether (Pa-Et), protoapigenone $1^{\prime}-O$-propylether (Pa-Pro), protoapigenone $1^{\prime}$-O-isopropylether (Pa-iPro), protoapigenone $1^{\prime}$-O-butylether
(Pa-But), protoapigenone $1^{\prime}-O$-allylether (Pa-All) and protoapigenone $1^{\prime}$-O-propargylether (Pa-PIII) were synthesized as previously described by Hunyadi et al. [17]. Human serum albumin (HSA), bovine serum albumin (BSA), Ochratoxin A (OTA), DMEM (Dulbecco's Modified Eagle's Medium) - high glucose ( $4500 \mathrm{mg} / \mathrm{L})$, fetal bovine serum (FBS), penicillin/streptomycin solution, adenosine $5^{\prime}$-triphosphate (ATP), propidium iodide (PI) (all from Sigma-Aldrich), FITC-Annexin V (from BD Pharmingen), apigenin (Api; from Fluka), ATP Bioluminescent Assay Kit CLS II (luciferine-luciferase; from Roche), fluorescamine (Fluram; from Serva), phenylmethylsulfonyl fluoride (PMSF; from Serva), calcein acetomethoxy ester (CAM; from Invitrogen) were used as received.

$2000 \mu \mathrm{M}$ stock solutions of flavonoid derivatives were prepared in dimethyl sulfoxide (DMSO; from Reanal, spectroscopic grade) and kept at $4{ }^{\circ} \mathrm{C}$, protected from light.

Phosphate buffered saline (PBS) containing $\mathrm{NaCl}(137 \mathrm{mM}), \mathrm{KCl}$ (2.7 mM), $\mathrm{NaH}_{2} \mathrm{PO}_{4}(8 \mathrm{mM}), \mathrm{K}_{2} \mathrm{HPO}_{4}(1.5 \mathrm{mM})$ in tridistilled water ( $\mathrm{pH}$ 7.4) was used to approximate physiological conditions.

\subsection{Instrumentation}

Fluorescence emission spectra and intensities were determined by a Hitachi F-4500 fluorescence spectrophotometer. A Berthold Lumat LB9507 luminometer was applied to quantify ATP concentrations. All analyses were performed in the presence of air at $+25^{\circ} \mathrm{C}$. Flow cytometry measurements were performed on Beckman-Coulter FC500 flow cytometer, data analysis was done using CXP software.

\subsection{Determination of binding constants of flavonoid-HSA complexes}

Binding constants $(K)$ of protoapigenone derivative - HSA complexes were determined by fluorescence quenching method. Increasing flavonoid concentrations $(0,1,2,3,4,5,7.5,10,12.5$, $15,20,30$, and $50 \mu \mathrm{M})$ were added to standard amounts of HSA $(5 \mu \mathrm{M})$ in PBS ( $\mathrm{pH} 7.4)$. Fluorescence emission spectra were recorded $\left(\lambda_{\text {exc }}=295 \mathrm{~nm}\right), K$ values of albumin-ligand complexes were evaluated with Hyperquad2006 software (Protonic Software) using the following equation, in which a 1:1 model is involved:

$$
\begin{aligned}
I= & I_{0}+\frac{\left(I_{H G}-I_{0}\right)}{2 \cdot[H]_{0}} \\
& \cdot\left([H]_{0}+[G]_{0}+\frac{1}{K}-\sqrt{\left([H]_{0}+[G]_{0}+\frac{1}{K}\right)^{2}-4 \cdot[H]_{0} \cdot[G]_{0}}\right)
\end{aligned}
$$

where $I$ denotes the fluorescence emission intensity of HSA at $336 \mathrm{~nm}$ in the presence of flavonoid; $I_{0}$ denotes the fluorescence emission intensity of HSA at $336 \mathrm{~nm}$ in the absence of flavonoid; $I_{H G}$ denotes the fluorescence emission intensity of pure flavonoidHSA complex (which is calculated by the Hyperquard2006); $\mathrm{K}$ denotes the binding constant; $[H]_{0}$ and $[G]_{0}$ denote the total concentrations of HSA and flavonoid, respectively.

Furthermore fluorescence quenching data were also evaluated using Stern-Volmer equation:

$\frac{F_{0}}{F}=1+K_{S V} *[Q]$

where $F_{0}$ and $F$ denote the steady-state fluorescence intensities of HSA in the absence and in the presence of the quencher, respectively. $K_{S V}$ (with the unit of $\mathrm{L} \mathrm{mol}^{-1}$ ) is the Stern-Volmer quenching constant and $[Q]$ is the concentration of the quencher (with the unit of $\left.\mathrm{mol} \mathrm{L}^{-1}\right)$. Stern-Volmer equation was applied for the determination of $K_{S V}$ by linear regression of $F_{0} / F$ against [ $Q$ ]. 


\subsection{Investigation of competing capacity}

Competing capacity of protoapigenone derivatives for HSA binding were quantified using the previously published fluorescence polarization based method $[6,18,19]$. Ochratoxin A (OTA) is a mycotoxin that binds to albumin with a very high affinity (the binding site is the same as seen for warfarin, being located in subdomain IIA, Site I) [20]. $25 \mu \mathrm{M}$ flavonoid concentrations were added to saturating OTA concentrations in the OTA-albumin model $\left(1 \mu \mathrm{M}\right.$ OTA and $1.7 \mu \mathrm{M}$ HSA in PBS, $\mathrm{pH} 7.4 ; \lambda_{\mathrm{exc}}=393 \mathrm{~nm}$, $\lambda_{\mathrm{em}}=446 \mathrm{~nm}$ ). HSA and Pa derivatives did not show any fluorescence signal at the applied wavelengths under these circumstances. The degree of fluorescence polarization was calculated as:

$P=\left(I_{v v}-G * I_{v h}\right) /\left(I_{v v}+G * I_{v h}\right)$

where $I_{v v}$ and $I_{v h}$ are fluorescence intensities measured at vertical excitation polarizer setting and at vertical and horizontal emission polarizer settings respectively, while $G$ is the actually measured optical correction factor. For calculating the degree of polarization 30 measuring points were averaged.

\subsection{Tissue cultures}

HepG2 (human liver cancer tissue, ATCC: HB-8065) adhering cells were cultured in DMEM (high glucose) with $10 \%$ FBS plus penicillin $(100 \mathrm{U} / \mathrm{mL})$ and streptomycin $(100 \mu \mathrm{g} / \mathrm{mL})$ in $25 \mathrm{~cm}^{2}$ sterile flasks (VWR), in 96-well and in 6-well sterile plastic plates (VWR). Cells were grown at $37{ }^{\circ} \mathrm{C}$ in the presence of $5 \% \mathrm{CO}_{2}$ in humidified atmosphere. Cells were pre-cultured for 2 days in the flasks then trypsinized and resuspended in fresh medium after having been counted in a Bürker type counting chamber. $1 \times 10^{4}$ cells per well in 96 -well $(150 \mu \mathrm{L}$ cell suspension in medium/well) plates and $1 \times 10^{5}$ cells per well in 6 -well plates (for flow cytometry; $2000 \mu \mathrm{L}$ cell suspension in medium/well) were plated. After $16 \mathrm{~h}$ the medium was replaced with fresh one and cells were treated with $10 \mu \mathrm{M}$ flavonoids. Measurements were done after 24-h incubation in the presence of flavonoids. All sterile work was carried out in an Aireguard-126300 (Nuaire) vertical laminar box.

\subsection{Cell number determination}

After subjecting cells to the appropriate treatment the medium was removed and cells were washed twice with $150 \mu \mathrm{L}$ PBS. Then cells were trypsinized using $50 \mu \mathrm{L}$ trypsin solution per well $(2.5 \mathrm{~g} / \mathrm{L}$ trypsin, $2.96 \mathrm{~g} / \mathrm{L}$ sodium citrate, $6 \mathrm{~g} / \mathrm{L}$ sodium chloride, $0.2 \mathrm{~g} / \mathrm{L}$ sodium EDTA). After 3 min incubation cells were counted in a standard Bürker-type counting chamber and data obtained for 9 readings were averaged.

\subsection{Calcein cell viability assay}

Calcein acetomethoxy ester (CAM) is transported into living cells where intracellular esterases cleave the ester bond resulting in the formation of the highly fluorescent calcein molecule that stays inside the cell [23]. After the removal of the medium cells were washed twice with $150 \mu \mathrm{L}$ PBS and treated with the dye $(150 \mu \mathrm{L} /$ well, $1.6 \mu \mathrm{M}$ CAM in PBS) for $60 \mathrm{~min}$ at $37^{\circ} \mathrm{C}$ in the dark. Then cells were washed with PBS and $150 \mu$ L lysis buffer (0.1\% SDS in PBS) was added to each well. After $10 \mathrm{~min}$ treatment at room temperature $100 \mu \mathrm{L}$ cell lysates were added to $2000 \mu \mathrm{L}$ PBS and fluorescence emission intensities were measured $\left(\lambda_{\mathrm{exc}}=480 \mathrm{~nm}, \lambda_{\mathrm{em}}=515 \mathrm{~nm}\right)$.

\subsection{Determination of intracellular ATP concentrations}

ATP concentrations were quantified using the firefly luciferinluciferase reaction. Sample preparation, measurements and data analyses were carried out by the method of Kőszegi et al. [21]. After two washing steps with PBS $150 \mu \mathrm{L}$ lysis buffer $(0.2 \mathrm{M}$ borate buffer, $10 \mathrm{mM} \mathrm{MgSO}_{4}, 0.1 \%$ Triton X 100, pH 9.2) was added to the wells and the plates were kept at room temperature for $15 \mathrm{~min} 50 \mu \mathrm{L}$ cell

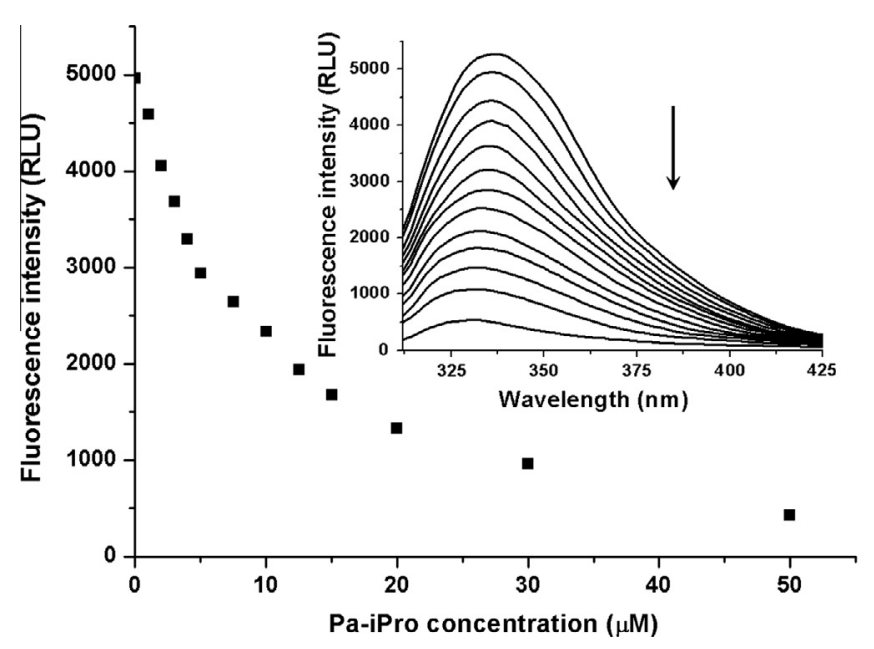

Fig. 2. Fluorescence emission spectra and intensities of HSA $(5 \mu \mathrm{M})$ in the absence and in the presence of increasing Pa-iPro concentrations (in PBS, pH 7.4; $\left.\lambda_{\mathrm{exc}}=295 \mathrm{~nm}, \lambda_{\mathrm{em}}=336 \mathrm{~nm}\right)$.

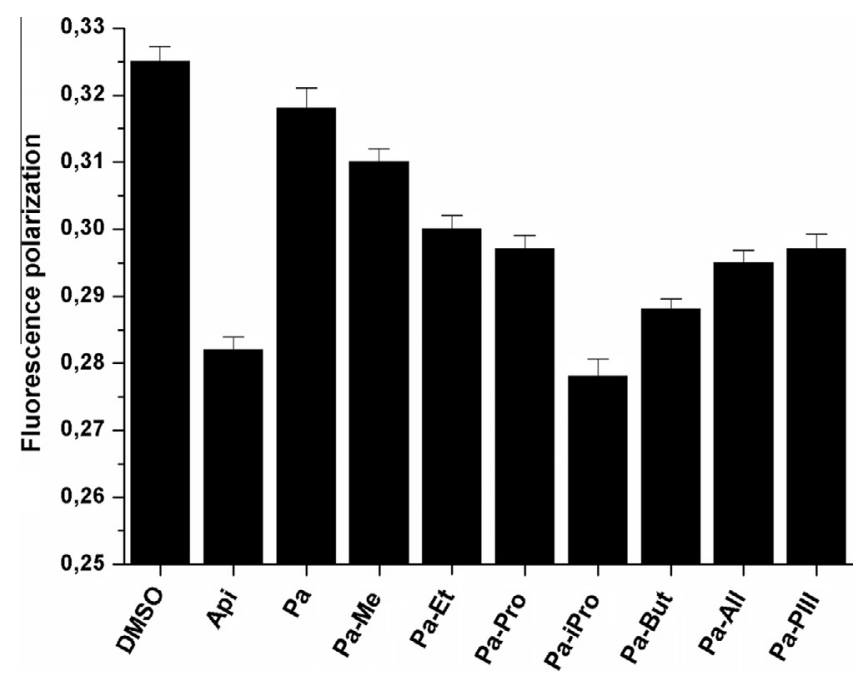

Fig. 3. Fluorescence polarization values \pm SD of OTA-HSA complex (OTA $1 \mu \mathrm{M}$, HSA $1.7 \mu \mathrm{M}$ ) in the absence and in the presence of $25 \mu \mathrm{M}$ of flavonoid derivatives. The decrease of fluorescence polarization data represents well the displacement of OTA from HSA by apigenin and protoapigenone derivatives $\left(\lambda_{\mathrm{exc}}=393 \mathrm{~nm}, \lambda_{\mathrm{em}}=446-\right.$ $\mathrm{nm} ; n=5)$.

Table 1

Binding constants calculated by Hyperquad2006 software $(K)$ with corresponding standard deviations (SDs) and Stern-Volmer quenching constants $\left(K_{S V}\right)$ of flavonoidHSA complexes.

\begin{tabular}{lll}
\hline Substance & $K\left(\mathrm{~L} \mathrm{~mol}^{-1}\right)$ & $K_{S V}\left(\mathrm{~L} \mathrm{~mol}^{-1}\right)$ \\
\hline Pa & $2.08 \times 10^{4}\left( \pm 0.22 \times 10^{4}\right)$ & $2.09 \times 10^{4}\left(R^{2}=0.991\right)$ \\
Pa-All & $3.56 \times 10^{4}\left( \pm 0.28 \times 10^{4}\right)$ & $3.49 \times 10^{4}\left(R^{2}=0.991\right)$ \\
Pa-Me & $3.89 \times 10^{4}\left( \pm 0.30 \times 10^{4}\right)$ & $3.96 \times 10^{4}\left(R^{2}=0.990\right)$ \\
Pa-Et & $4.26 \times 10^{4}\left( \pm 0.26 \times 10^{4}\right)$ & $4.31 \times 10^{4}\left(R^{2}=0.992\right)$ \\
Pa-But & $5.14 \times 10^{4}\left( \pm 0.18 \times 10^{4}\right)$ & $4.86 \times 10^{4}\left(R^{2}=0.998\right)$ \\
Pa-PIII & $5.34 \times 10^{4}\left( \pm 0.25 \times 10^{4}\right)$ & $5.03 \times 10^{4}\left(R^{2}=0.996\right)$ \\
Pa-Pro & $6.19 \times 10^{4}\left( \pm 0.39 \times 10^{4}\right)$ & $6.28 \times 10^{4}\left(R^{2}=0.990\right)$ \\
Pa-iPro & $1.70 \times 10^{5}\left( \pm 0.59 \times 10^{4}\right)$ & $1.34 \times 10^{5}\left(R^{2}=0.992\right)$ \\
Api & $2.08 \times 10^{5}\left( \pm 1.94 \times 10^{4}\right)$ & $1.46 \times 10^{5}\left(R^{2}=0.991\right)$ \\
\hline
\end{tabular}


lysates were pipetted into disposable $75 * 12 \mathrm{~mm}$ plastic cuvettes (Sarstedt) containing $100 \mu \mathrm{L}$ luciferin-luciferase reagent and $850 \mu \mathrm{L}$ ATP measuring buffer (0.1 M TRIS-acetate buffer, $10 \mathrm{mM}$ $\mathrm{MgSO}_{4}, 2$ mM Na${ }_{2}$ EDTA, $0.1 \%$ Triton X 100, pH 7.75). ATP concentrations were determined with internal standardization [21].

\subsection{Determination of total protein concentrations}

Fluorescamine (Fluram) reacts selectively with primary amines and forms a highly fluorescent product therefore it is used to determine total protein levels [22]. Protein determination was done from the same samples that were applied for ATP detection (lysis with Triton-borate buffer). To prevent proteolysis the lysis buffer contained $0.2 \mathrm{mM}$ PMSF (serine protease inhibitor). $75 \mu \mathrm{L}$ lysates were added to $2000 \mu \mathrm{L}$ borate buffer $(0.2 \mathrm{M}, \mathrm{pH} 9.2)$ thereafter $50 \mu \mathrm{L}$ fluorescamine solutions ( $10 \mathrm{mM}$; in DMSO) were vigorously mixed with the system. Then BSA-related protein concentrations were calculated from fluorescence emission intensities $\left(\lambda_{\mathrm{exc}}=394\right.$ $\left.\mathrm{nm}, \lambda_{\mathrm{em}}=475 \mathrm{~nm}\right)$.

\subsection{Annexin $V$ and propidium iodide staining assay}

After subjecting cells to the appropriate treatment the medium containing any detached cells was carefully removed and spared for later analysis. Attached cells were rinsed with PBS and trypsinized then combined with the previously removed medium. After pulse centrifugation for $3 \mathrm{~s}$ at 14,000 rpm, cell pellet was resuspended in binding buffer (10 mM HEPES, $140 \mathrm{mM} \mathrm{NaCl}, 2.5 \mathrm{mM}$ $\mathrm{CaCl}_{2}, \mathrm{pH} 7.4$ ) and incubated for $15 \mathrm{~min}$, at room temperature, in the dark with $5 \mu \mathrm{L}$ of FITC-Annexin V reagent and $10 \mu \mathrm{L}$ of PI solution $(50 \mu \mathrm{g} / \mathrm{mL})$. Then cells were analyzed by flow cytometry.

\subsection{Cell cycle analyses}

Cells were washed with $1 \mathrm{~mL}$ cold PBS then trypsinized and centrifuged for $3 \mathrm{~s}$ at $14,000 \mathrm{rpm}$. The supernatant was removed and after a washing step with PBS the pellet was resuspended in $90 \%$ ethanol. After an additional washing step with PBS the cells were treated with $500 \mu \mathrm{L}$ PI solution $(50 \mu \mathrm{g} / \mathrm{mL}$ propidium iodide, $1 \mathrm{mg} / \mathrm{mL}$ RNase A, $0.1 \%$ Triton X 100 in PBS) for $30 \mathrm{~min}$, at room temperature, in the dark. $1 \times 10^{4}$ cells were analyzed by flow cytometry.

\subsection{Statistics}

The data represent mean \pm SD from minimum of 3 independent experiments. Statistical analysis was performed by One-Way
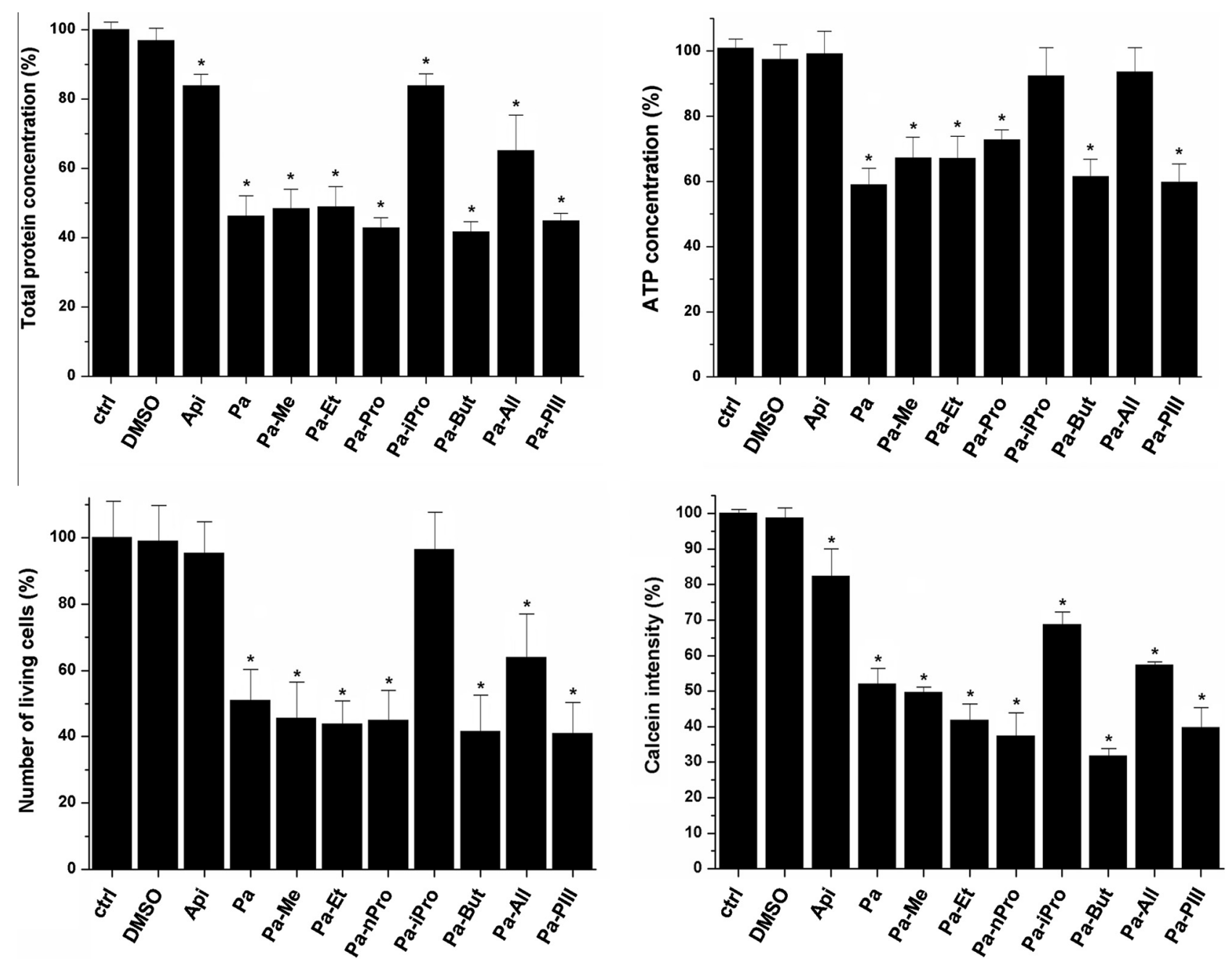

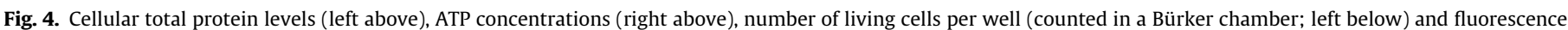

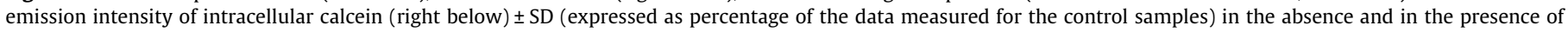
$10 \mu \mathrm{M}$ apigenin or protoapigenone derivatives $(P<0.05)$. 
ANOVA test using IBM SPSS Statistics (Version 20). The level of significance was set at $P<0.05$.

\section{Results}

\subsection{Albumin binding and competing capacity of flavonoids}

The high quenching effect on tryptophan residue (Fig. 2) caused by the flavonoid derivatives and their ability to displace OTA from the surface of HSA (Fig. 3) strongly support our hypothesis that protoapigenone derivatives - similarly to other flavonoid aglycones - also occupy subdomain IIA on human albumin. Determined binding constants (Table 1) show that protoapigenone binds with a significantly lower affinity to HSA than apigenin. $1^{\prime}$-O-alkyl substitution of protoapigenone results in the increase of $K$ values. Pa-iPro represents similar degree in binding affinity as compared to that of apigenin, suggesting that not only the lipophilicity but also the steric properties are important factors for albumin binding. Interestingly, the allyl derivative shows the weakest interaction, while isopropyl, propyl and propargyl derivatives show the strongest binding capacities.

In the next series of experiments displacing ability of Pa derivatives were investigated against ochratoxin A using fluorescence polarization technique. Measurements were done at the fluorescence excitation and emission wavelength maxima of the albumin-bound OTA. Since the wavelength maxima of free and albumin-bound OTA are very similar $[6,19]$ we can determine only the sum of their fluorescence signal and an average degree of polarization of the system. Whereas fluorescence polarization $(P)$ values are based on the rotational freedom of the molecule, the free form has a very low $(P=0.012)$ and the albumin-bound form has a high $(P=0.325) P$ value. Therefore a decrease in the fluorescence polarization data indicates the desorption of OTA from the surface of HSA. Displacement investigations (Fig. 3) suggest similar results then stability constants: Pa is the weakest competitor while Pa-iPro is characterized to have the most potent displacing ability. On the other hand Pa-All represents similar efficiency to Pa-Pro and Pa-PIII as a competitor.

\subsection{Influence on ATP and protein levels}

Significant changes of intracellular ATP concentrations were observed after 24-h treatment with Pa (Fig. 4). Api, Pa-iPro and Pa-All did not cause considerable changes in ATP levels (compared to the control). Other protoapigenone derivatives show similar or slightly weaker effect than Pa.

Total protein level falls to $46 \%$ of the initial value after Pa treatment. Api, Pa-iPro and Pa-All represent again their weaker impact but all other derivatives show similar results (there are no significant differences; $P<0.05$ ) compared to Pa (Fig. 4).

\subsection{Influence on cell viability}

In order to determine the effect of protoapigenone derivatives on cell viability, cells were counted in a Bürker chamber furthermore calcein and Annexin V/PI viability assays were also performed. After 24-h treatment with $10 \mu \mathrm{M}$ of $\mathrm{Pa}$, the number of living cells was less than $50 \%$ of the control (Fig. 4). Api and Pa-iPro did not cause significant changes (compared to the control), and the decrease exerted by Pa-All was considerably milder than that
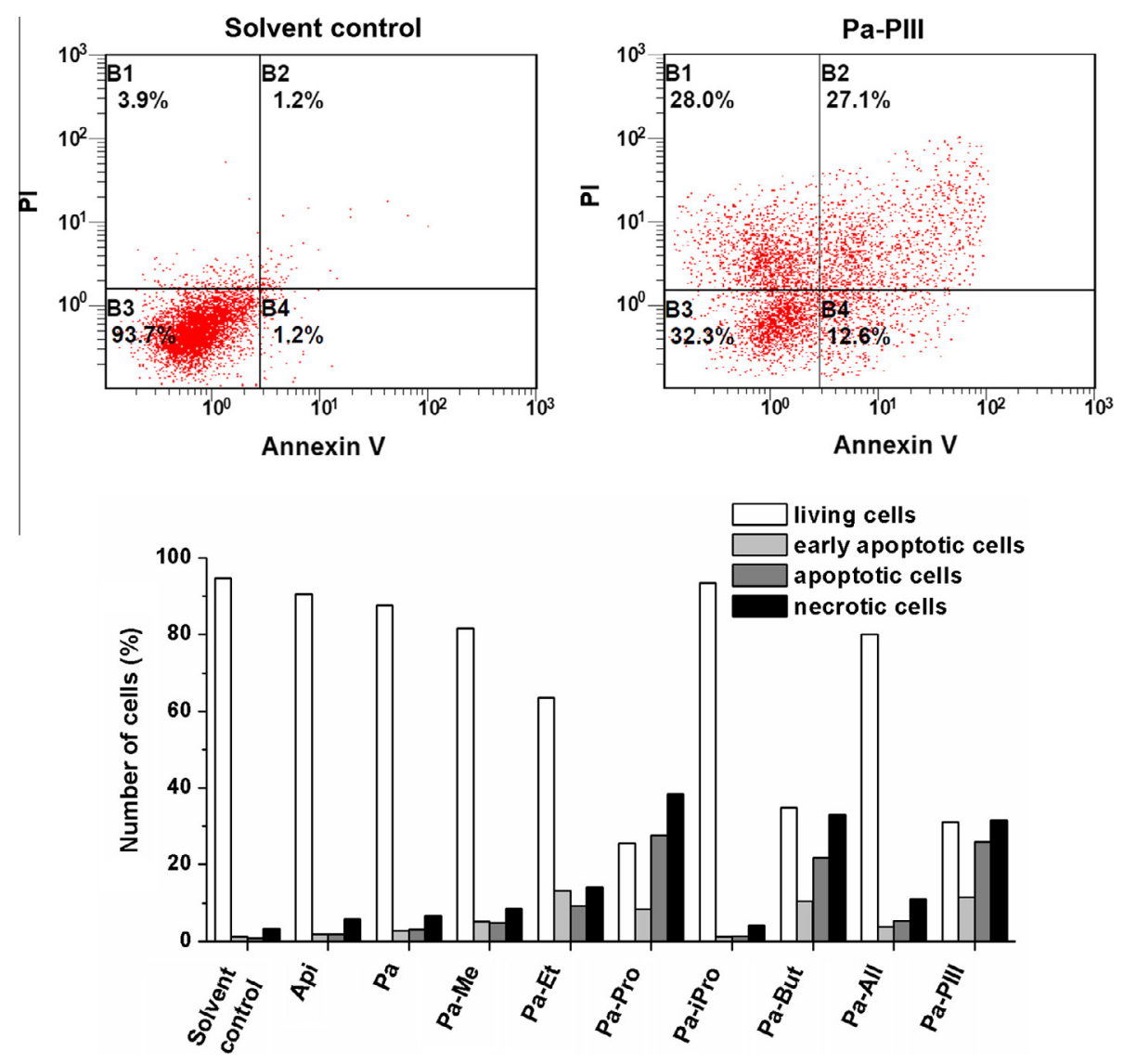

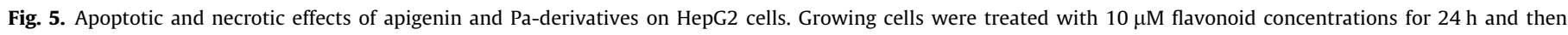
stained with Annexin V/PI for flow cytometry analysis (SD values did not exceed 5\%). 
of the other derivatives. In other cases treatment with $1^{\prime}$-O-alkylated $\mathrm{Pa}$ derivatives resulted in a stronger (but not significant; $P<0.05)$ negative effect (cytotoxicity) on cell viability than Pa.

Calcein data show similar tendency when compared to cell count measurements (Fig. 4). Api and Pa-iPro exert a significantly weaker toxic effect than Pa; but in contrast to the previous cell count results both agents caused significant decrease in cell viability compared to the control. Pa-All represents lower, while other derivatives indicate higher impact on viability of HepG2 cells, nevertheless only Pa-But caused significant decrease of calcein intensity compared to $\mathrm{Pa}(P<0.05)$. Fig. 4 shows that $1^{\prime}-O-$ alkylation (with the exception of Pa-iPro and Pa-All) causes detectable decrease in cell viability, suggesting that an increase in the length of the carbon chain slightly elevates the cytotoxic phenomenon.

In the next series of experiments Annexin V/PI staining assay was applied to investigate the potential apoptotic and necrotic effects of tested flavonoid derivatives. Fig. 5 represents that Api, Pa and Pa-iPro treatment caused only a minor decrease in the number of living cells and also just a slight elevation of apoptotic and necrotic cells were observed. On the other hand $1^{\prime}$-O-alkyl derivatives caused a significant decrease in living cell number and a considerable increase in both apoptotic and necrotic cell fractions were observed. These results clearly demonstrate that a longer aliphatic side-chain leads to a considerable increase in apoptotic and necrotic effects; but interestingly Pa-Pro, Pa-PIII and Pa-But show almost the same impact. Furthermore Annexin V/PI assay also supports that Pa-All exerts much less antitumor activity than Pa-Pro or Pa-PIII. Our results strongly suggest that both apoptotic and necrotic effects play a key role in the mode of action of Paderivatives.

\subsection{Effects on cell cycle}

Finally impacts of Pa-derivatives on cell cycle were also examined. In the case of Api the elevation in the number of G2/M phase cells was observed on the other hand Fig. 6 suggests that $24 \mathrm{~h}$ treatment with $10 \mu \mathrm{M}$ Pa results in both S and G2/M phase cell cycle arrest. Pa-iPro treatment supports again the previously experienced no significant impacts. All $1^{\prime}$-O-alkyl Pa derivatives (with the exception of Pa-iPro) show a SubG1 peak which is one of the reliable biochemical markers of apoptosis [24]; this effect is the highest in the case of Pa-Et and Pa-Me derivatives. Pa-All shows again weaker impact but the SubG1 phase is also visible. These results are in good agreement with the data obtained for the Annexin V/ PI assay. Besides induction of SubG1 phase Pa-Et, Pa-Pro, Pa-But and Pa-PIII show the most strongest G2/M phase cell cycle arrest.

\section{Discussion}

In order to get deeper insight into the biological behavior of the flavonoid protoapigenone and its $1^{\prime}-O$-alkyl derivatives albumin binding properties and several biological effects on HepG2 cells were investigated. In our experiments results obtained from calculation of stability constants (using Hiperquad2006 software and also Stern-Volmer equation) for protoapigenone and its derivatives were completed with the data on competitive investigations with ochratoxin A. Application of Stern-Volmer plot suggests a static quenching of fluorescence intensity of HSA by Pa-derivatives (results in Table 1). After 24-h treatment of HepG2 cells with $10 \mu \mathrm{M}$ flavonoid derivatives ATP and total protein levels were quantified, cell number was counted, calcein and Annexin V/PI viability assays were done furthermore effects on cell cycle were also
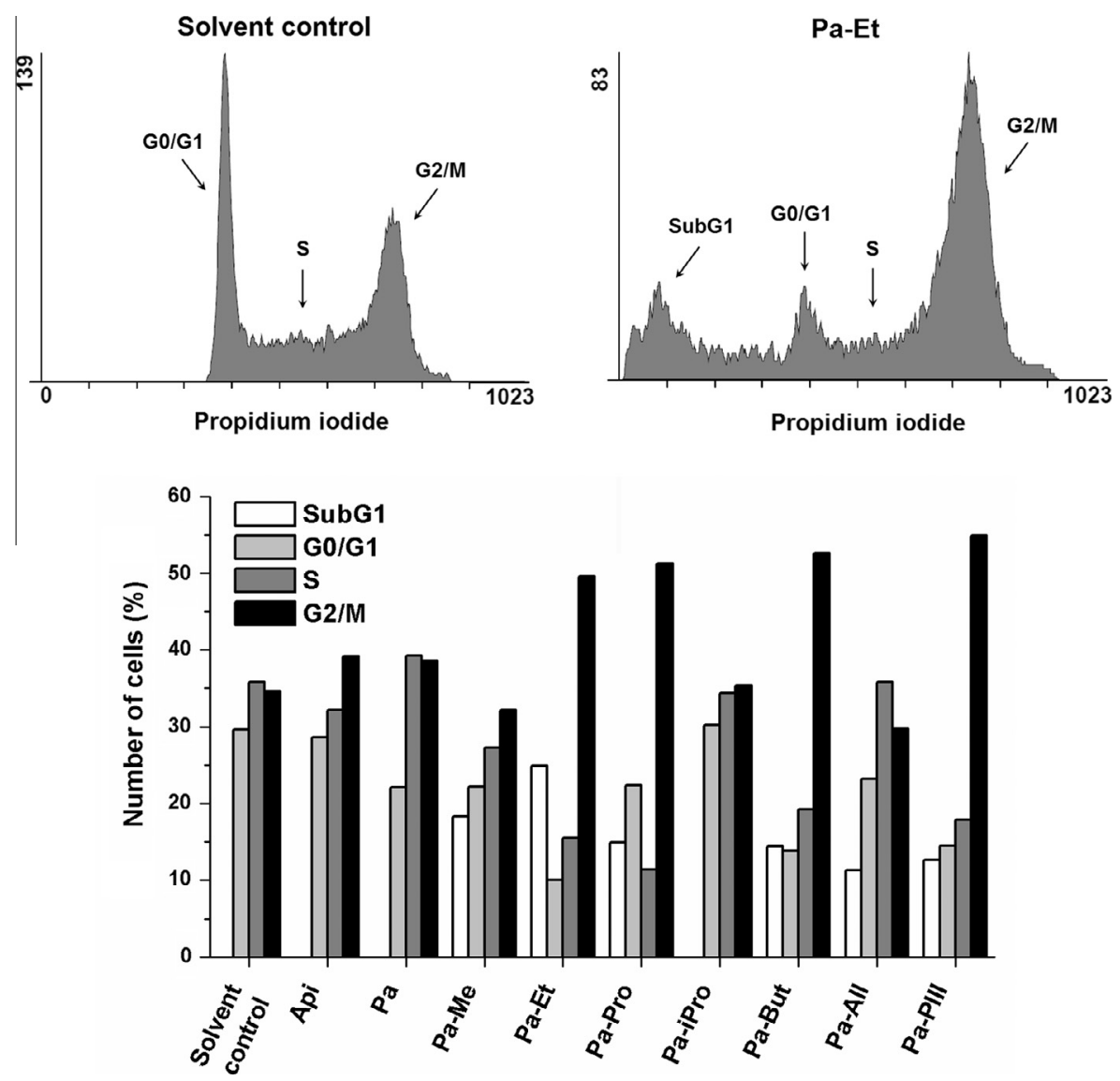

Fig. 6. Effect of $10 \mu \mathrm{M}$ apigenin and Pa-derivatives on cell cycle of HepG2 cells after a 24-h treatment with a single dose of flavonoid (SD values did not exceed 6\%). 
investigated. Our results represent well that stability constants of Pa derivative - HSA complexes and competing capacities of different alkyl derivatives show major differences among the studied flavonoids. Furthermore it can also be stated that by increasing the length of the carbon chain a considerable increase in stability constants is observed, these results are in good accordance with the competing capacity of the studied substances against OTA.

Our findings show that protoapigenone treatment causes a major decrease in cell viability, and a similar tendency was observed in the case of $1^{\prime}-O$-alkyl derivatives with the exception of isopropyl derivative representing much less effects (the allyl derivative also exerts milder impacts). $10 \mu \mathrm{M}$ of Pa almost halved the number of living cells, cell viability and intracellular ATP levels, moreover, total protein concentrations fell down to $46 \%$ of the control. Treatment with 1 '-O-alkylated derivatives, again with the exceptions of Pa-iPro and Pa-All, resulted in similar or slightly stronger effects. On the other hand Annexin V/PI assay and cell cycle analyses show major differences between Pa-derivatives: the longer aliphatic side-chain causes significantly stronger impacts on HepG2 cells, the primarily most effective derivatives are Pa-Pro, Pa-But and Pa-PIII.

Our investigation proves that changing the lipophilicity and plasma protein binding properties causes a consequent alteration in the pharmacokinetic behavior of Pa without decreasing its pharmacodynamic effect. Moreover, parallel with the structural modifications additional minor or major increase in the biological effects were also detectable. These latter findings are in good agreement with our previously suggested structure-activity relationships for the $1^{\prime}$ - $O$-alkylated Pa derivatives, namely a longer, aliphatic sidechain might lead to an increase in anticancer activity, while a branching side-chain, as in case of Pa-iPro can considerably decrease it [17]. Moreover, the alkylated pharmacophore B-ring represents much higher chemical stability as compared to that of the non-substituted one, such as in the case of protoflavonoid Pa. Since current studies suggest that the described anticancer effects show tumor selective property $[8,11,13,14]$ some of these compounds might become first-line candidates in the ongoing development of a practically novel class of anticancer agents. Investigating the in vivo significance of albumin binding in the pharmacokinetics of these compounds is certainly an urgent need in this process, such experiments are going to be performed and reported in the near future.

\section{Acknowledgements}

Financial support of the Developing Competitiveness of Universities in the South Transdanubian Region project (SROP-4.2.1.B-10/ 2/KONV-2010-0002) is highly appreciated. The work presented here was performed within the framework of COST Action CM0804, Chemical Biology with Natural Products, and it was supported by the New Hungary Development Plan (TÁMOP 4.2.1/B-09/ $1 /$ KONV-2010-0005), the National Science Council, Executive Yuan, Taiwan (NSC 101-2325-B-039-004). The publication is supported by the European Union and co-funded by the European Social Fund (TÁMOP-4.2.2/B-10/1-2010-0012).

\section{References}

[1] B.H. Havsteen, The biochemistry and medical significance of the flavonoids, Pharmacol. Therap. 96 (2002) 67-202.

[2] C.C. Wong, N.P. Botting, C. Orfila, N. Al-Maharik, G. Williamson, Flavonoid conjugates interact with organic anion transporters (OATs) and attenuate cytotoxicity of adefovir mediated by organic anion transporter 1 (OAT1/ SLC22A6), Biochem. Pharmacol. 81 (2011) 942-949.
[3] J. Bektic, R. Guggenberger, B. Spengler, V. Christoffel, A. Pelzer, A.P. Berger, R. Ramonera, G. Bartsch, H. Klocker, The flavonoid apigenin inhibits the proliferation of prostatic stromal cells via the MAPK-pathway and cell-cycle arrest in G1/S, Maturitas 55 (2006) 37-46.

[4] E. Youl, G. Bardy, R. Magous, G. Cros, F. Sejalon, A. Virsolvy, S. Richard, J.F. Quignard, R. Gross, P. Petit, D. Bataille, C. Oiry, Quercetin potentiates insulin secretion and protects INS-1 pancreatic $\beta$-cells against oxidative damage via the ERK1/2 pathway, Br. J. Pharmacol. 161 (2010) 799-814.

[5] J. Xiao, Y. Zhao, H. Wang, Y. Yuan, F. Yang, C. Zhang, K. Yamamoto, Noncovalent interaction of dietary polyphenols with common human plasma proteins, J. Agric. Food Chem. 59 (2011) 10747-10754.

[6] M. Poór, S. Kunsági-Máté, T. Bencsik, J. Petrik, S. Vladimir-Knežević, T. Kőszegi, Flavonoid aglycones can compete with ochratoxin A for human serum albumin: a new possible mode of action, Int. J. Biol. Macromol. 51 (2012) 279-283.

[7] A.S. Lin, F.R. Chang, C.C. Wu, C.C. Liaw, Y.C. Wu, New cytotoxic flavonoids from Thelypteris torresiana, Planta Med. 71 (2005) 867-870.

[8] H.L. Chang, J.H. Su, Y.T. Yeh, Y.C. Lee, H.M. Chen, Y.C. Wu, S.S. Yuan, Protoapigenone, a novel flavonoid, inhibits ovarian cancer cell growth in vitro and in vivo, Cancer Lett. 267 (2008) 85-95.

[9] H.L. Chang, Y.C. Wu, J.H. Su, Y.T. Yeh, S.S. Yuan, Protoapigenone, a novel flavonoid, induces apoptosis in human prostate cancer cells through activation of p38 mitogen-activated protein kinase and c-Jun $\mathrm{NH}_{2}$-terminal kinase $1 / 2, \mathrm{~J}$. Pharmacol. Exp. Ther. 325 (2008) 841-849.

[10] W.Y. Chen, Y.A. Hsieh, C.I. Tsai, Y.F. Kang, F.R. Chang, Y.C. Wu, C.C. Wu, Protoapigenone, a natural derivative of apigenin, induces mitogen-activated protein kinase-dependent apoptosis in human breast cancer cells associated with induction of oxidative stress and inhibition of glutathione S-transferase $\pi$, Invest. New Drugs 29 (2011) 1347-1359.

[11] B. Danko, A. Martins, D.W. Chuang, H.C. Wang, L. Amaral, J. Molnár, F.R. Chang, Y.C. Wu, A. Hunyadi, In vitro cytotoxic activity of novel protoflavone analogs selectivity towards a multidrug resistant cancer cell line, Anticancer Res. 32 (2012) 2863-2870.

[12] C.P. Tung, F.R. Chang, Y.C. Wu, D.W. Chuang, A. Hunyadi, S.T. Liu, Inhibition of the Epstein-Barr virus lytic cycle by protoapigenone, J. Gen. Virol. 92 (2011) 1760-1768.

[13] Q. Yuan, Z. Liu, C. Xiong, L. Wu, J. Wang, J. Ruan, A novel, broad-spectrum antitumor compound containing the 1-hydroxycyclohexa-2,5-dien-4-one group: the disclosure of a new antitumor pharmacophore in protoapigenone 1, Bioorg. Med. Chem. Lett. 21 (2011) 3427-3430.

[14] Q. Yuan, S. Cai, X. Zhang, Z. Liu, Z. Li, X. Luo, C. Xiong, J. Wang, J. Hub, J. Ruan, A new protoapigenone analog RY10-4 induces apoptosis and suppresses invasion through the PI3K/Akt pathway in human breast cancer, Cancer Lett. 324 (2012) 210-220.

[15] H.M. Chen, F.R. Chang, Y.C. Hsieh, Y.J. Cheng, K.C. Hsieh, L.M. Tsai, A.S. Lin, Y.C. Wu, S.S. Yuan, A novel synthetic protoapigenone analogue, WYC02-9, induces DNA damage and apoptosis in DU145 prostate cancer cells through generation of reactive oxygen species, Free Radical. Biol. Med. 50 (2011) 1151-1162.

[16] H.C. Wang, A.Y. Lee, W.C. Chou, C.C. Wu, C.N. Tseng, K.Y. Liu, W.L. Lin, F.R. Chang, D.W. Chuang, A. Hunyadi, Y.C. Wu, Inhibition of ATR-dependent signaling by protoapigenone and its derivative sensitizes cancer cells to interstrand cross-link-generating agents in vitro and in vivo, Mol. Cancer Ther. 11 (2012) 1443-1453.

[17] A. Hunyadi, D.W. Chuang, B. Danko, M.Y. Chiang, C.L. Lee, H.C. Wang, C.C. Wu, F.R. Chang, Y.C. Wu, Direct semi-synthesis of the anticancer lead-drug protoapigenone from apigenin, and synthesis of further new cytotoxic protoflavone derivatives, PLOS One 6 (2011) e23922.

[18] Y.V. Il'ichev, J.L. Perry, F. Rüker, M. Dockal, J.D. Simon, Interaction of ochratoxin A with human serum albumin. Binding sites localized by competitive interactions with the native protein and its recombinant fragments, Chem. Biol. Interact. 141 (2002) 275-293.

[19] M. Poór, S. Kunsági-Máté, Z. Czibulya, Y. Li, B. Peles-Lemli, J. Petrik, S. VladimirKnežević, T. Köszegi, Fluorescence spectroscopic investigation of competitive interactions between ochratoxin $\mathrm{A}$ and 13 drug molecules for binding to human serum albumin, Luminescence (2012), http://dx.doi.org/10.1002/ bio. 2423.

[20] J.L. Perry, M.R. Goldsmith, M.A. Peterson, D.N. Beratan, G. Wozniak, F. Ru1ker, J.D. Simon, Structure of the ochratoxin A binding site within human serum albumin, J. Phys. Chem. B 108 (2004) 16960-16964.

[21] T. Kőszegi, J. Petrik, S. Vladimir- Knežević, S. Nagy, Co-determination of ATP and proteins in Triton X 100 non-ionic detergent-opened monolayer cultured cells, Luminescence 22 (2007) 415-419.

[22] T. Bantan-Polak, M. Kassai, K.B. Grant, A comparison of fluorescamine and naphthalene-2,3-dicarboxaldehyde fluorogenic reagents for microplate-based detection of amino acids, Anal. Biochem. 297 (2001) 128-136.

[23] E. Bell, X. Cao, J.A. Moibi, S.R. Greene, R. Young, M. Trucco, Z. Gao, F.M. Matschinsky, S. Deng, J.F. Markman, A. Naji, B.A. Wolf, Rapamycin has a deleterious effect on MIN-6 cells and rat and human islets, Diabetes 52 (2003) 2731-2739.

[24] J. Liu, Y. Wang, J. Cui, L. Xing, H. Shen, S. Wu, H. Lian, J. Wang, X. Yan, X. Zhang, Ochratoxin A induces oxidative DNA damage and G1 phase arrest in human peripheral blood mononuclear cells in vitro, Toxicol. Lett. 211 (2012) 164-171. 


\title{
Discovery of the first non-planar flavonoid that can strongly inhibit xanthine oxidase: protoapigenone 1'-0-propargyl ether
}

\author{
Attila Hunyadi ${ }^{\text {a,* }}$, Ana Martins ${ }^{\text {a,b,c }}$, Balazs Danko ${ }^{\text {a,d }}$, Da-Wei Chuang ${ }^{\text {d }}$, Patrick Trouillas e,f,g, \\ Fang-Rong Chang ${ }^{\mathrm{d}, \mathrm{h}, \mathrm{i}}$, Yang-Chang $\mathrm{Wu}^{\mathrm{j}, \mathrm{k}}$, George Falkay ${ }^{1}$ \\ anstitute of Pharmacognosy, University of Szeged, Eötvös u. 6, Szeged 6720, Hungary \\ ${ }^{\mathrm{b}}$ Department of Medical Microbiology and Immunobiology, University of Szeged, Dóm tér 9, Szeged 6720, Hungary \\ ' Unidade de Parasitologia e Microbiologia Médica, Instituto de Higiene e Medicina Tropical, Universidade Nova de Lisboa, Rua da Junqueira 100, Lisbon 1349-008, Portugal \\ ${ }^{\mathrm{d}}$ Graduate Institute of Natural Products, Kaohsiung Medical University, Shih-Chuan 1st Rd. 100, Kaohsiung 80708, Taiwan, ROC \\ e Laboratoire de Chimie des Substances Naturelles EA-1069, Faculté de Pharmacie, Université de Limoges, 2 rue du Docteur Marcland, Limoges CEDEX 87000, France \\ ${ }^{\mathrm{f}}$ Service de Chimie des Matériaux Nouveaux, Université de Mons-UMONS, Place du Parc 20, Mons 7000, Belgium

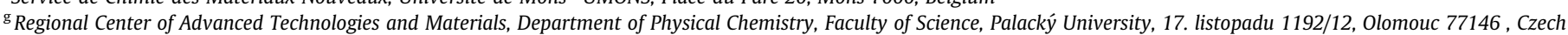 \\ Republic \\ ${ }^{\mathrm{h}}$ Cancer Center, Kaohsiung Medical University Hospital, Shih-Chuan 1st Rd. 100, Kaohsiung 80708, Taiwan, ROC \\ ${ }^{i}$ RED Center of Chinese Herbal Medicines E New Drugs, College of Pharmacy, Kaohsiung Medical University, Shih-Chuan 1st Rd. 100, Kaohsiung 80708, Taiwan, ROC \\ ${ }^{j}$ School of Pharmacy, College of Pharmacy, China Medical University Hospital, China Medical University, Hsueh-Shih Rd. 91, Taichung 404, Taiwan, ROC

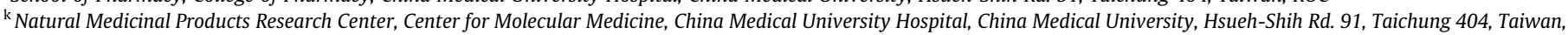 \\ $R O C$ \\ ${ }^{1}$ Department of Pharmacodynamics and Biopharmacy, University of Szeged, Eötvös u. 6, Szeged 6720, Hungary
}

\section{A R T I C L E I N F O}

Article history:

Received 5 June 2013

Revised 5 September 2013

Accepted 20 September 2013

Available online 27 September 2013

\section{Keywords:}

Xanthine oxidase inhibitor

Flavonoid

Protoflavone

Protoapigenone

Structure-activity relationships

Docking study

\begin{abstract}
A B S T R A C T
Xanthine oxidase (XO) is a key enzyme in purine metabolism with an important role in various pathologies. Several flavonoids have been reported for their capacity to inhibit this enzyme, and, for these compounds, the ability to adopt a planar 3D structure has been accepted as fundamental prerequisite for such activity. Here we report the in vitro investigation of a series of non-planar protoflavone derivatives as XO inhibitors, among which protoapigenone 1 '-O-propargyl ether was found to be an efficient competitive inhibitor of the enzyme with an IC $\mathrm{I}_{50}$ value of $3.61 \mu \mathrm{M}$, significantly $(p<0.001)$ stronger than the anti-gout drug allopurinol $\left(\mathrm{IC}_{50}=8.72 \mu \mathrm{M}\right)$. Methoxy substitution at $\mathrm{C}-7$, however, resulted in complete loss of activity. In silico docking supported the observed structure-activity relationships, based on which a 'planar structure' itself can no longer be considered as a criterion for flavonoid-type inhibitors of XO.
\end{abstract}

(c) 2013 Elsevier Ltd. All rights reserved.
Xanthine oxidase (XO), a molybdenum-containing metalloenzyme, is well known for its key role in the catabolism of purines, during which hypoxanthine and xanthine are transformed into uric acid. Elevated levels of uric acid lead to various pathological events including gout and the related formation of kidney stones. Moreover, although uric acid is the most abundant antioxidant in the human body, high XO activity makes a major contribution to oxidative stress by also producing reactive oxygen species (ROS) according to the following equations:

Xanthine $+\mathrm{H}_{2} \mathrm{O}+\mathrm{O}_{2} \rightarrow$ Uric acid $+\mathrm{H}_{2} \mathrm{O}_{2}$

Xanthine $+\mathrm{H}_{2} \mathrm{O}+\mathrm{O}_{2} \rightarrow$ Uric acid $+\mathrm{O}_{2}^{\cdot-}+2 \mathrm{H}^{+}$

Possibly related to its role in oxidative stress, $\mathrm{XO}$ inhibition also seems to have benefits in many pathological conditions including

\footnotetext{
* Corresponding author. Tel.: +36 62546456; fax: +36 62545704 .

E-mail address: hunyadi.a@pharm.u-szeged.hu (A. Hunyadi).
}

tissue injuries of various types and inflammatory diseases, and might also decrease cardiovascular risk. ${ }^{2,3}$ Despite their various problematic adverse effects, the purine derivative allopurinol and its active metabolite, oxipurinol, have long been nearly the exclusive choices for therapeutic XO inhibition. Thanks to the significant efforts on the search for new, non-purine type XO inhibitors, several different classes of compounds have been revealed as possible alternatives, ${ }^{4,5}$ a number of which have also been patented. ${ }^{6}$ Febuxostat [2-(3-cyano-4-isobutoxyphenyl)-4-methyl-1,3-thiazole-5-carboxylic acid], a non-competitive inhibitor of the enzyme, is probably the most important example to mention due to its recent approval by the FDA. ${ }^{7}$

Another prospective class of non-purine type XO inhibitors is the flavonoids, many of which have long been described as efficient inhibitors of the enzyme. ${ }^{8}$ In fact, this enzyme-inhibiting activity is also related to their well-known antioxidant action in vivo, ${ }^{9}$ potentially contributing to the widely accepted, generally positive 
effect of flavonoids on human health. A large body of scientific literature on flavonoids as XO inhibitors has been published. All established structure-activity relationships (SAR) of these compounds are highly consistent with the following conclusions: (i) the structures of efficient inhibitors allow them to take a planar shape (i.e., no $\mathrm{sp}^{3}$ carbons are present; flavones are far more active than flavanones), (ii) OH-groups at C-5 and C-7 are important for strong activity, and (iii) flavonols, isoflavones and anthocyanodins are less active than flavones. ${ }^{10-14}$ The structures of apigenin, a well-known, highly potent flavone-type XO inhibitor, and naringenin, an inactive flavanone, are shown in Figure 1, as well as that of quercetin, another efficient inhibitor of the enzyme, whose 3D structure has recently been revealed within the active center of XO.

Protoflavones are rare and unusual flavonoid derivatives having a non-aromatic B-ring; in Nature they can most typically be found in certain genera of ferns, for example, Pseudophegopterys or Thelypteris. ${ }^{15}$ These compounds have become a focus of biomedical research, and some are currently under development as promising anticancer agents. ${ }^{15}$ As part of our ongoing research, we have started a thorough investigation on the redox properties of protoflavone derivatives synthesized previously. ${ }^{16,17}$ Screening their capacity for XO inhibition led to surprising results that partially re-write the accepted SAR of flavonoids in this regard (Fig. 2), as presented and discussed below.

The genkwanin derivatives (group B) were inactive, whilst weak inhibition was found for some of the naphthoflavone derivatives (group C), and weak to moderate activity was observed for most of the protoapigenone analogs (group A). However, compound 7, protoapigenone 1'-O-propargyl ether, was found to inhibit the enzyme almost completely at the tested concentration. The dose-effect curve was subsequently determined for this compound and compared to those of allopurinol and apigenin (Fig. 3).

In order to investigate thoroughly the inhibition mechanism of 7, the enzyme kinetics were studied. The kinetic curve was found to be characteristic of substrate inhibition (Fig. 4), that is, increasing the amounts of the substrate (here: xanthine) could also exert mild inhibition. Such a situation has already been described for XO. ${ }^{18}$ Furthermore, a global model with identical $V_{\max }$ values for each curve was found $\left(1.376 \mathrm{nM} \mathrm{s}^{-1}\right)$ at $p<0.05$, while no solutions were found when curve fitting criterion of a common $K_{\mathrm{m}}$ value was set for all curves. Based on these thorough analyses, compound 7 was strongly suggested to be a competitive inhibitor of the enzyme.

The binding mode of $\mathbf{7}$ into the enzyme was investigated by in silico docking, using the $3 \mathrm{NVY}$ structure, corresponding to the crystal structure of $\mathrm{XO}$ in complex with quercetin (www.pdb.org).

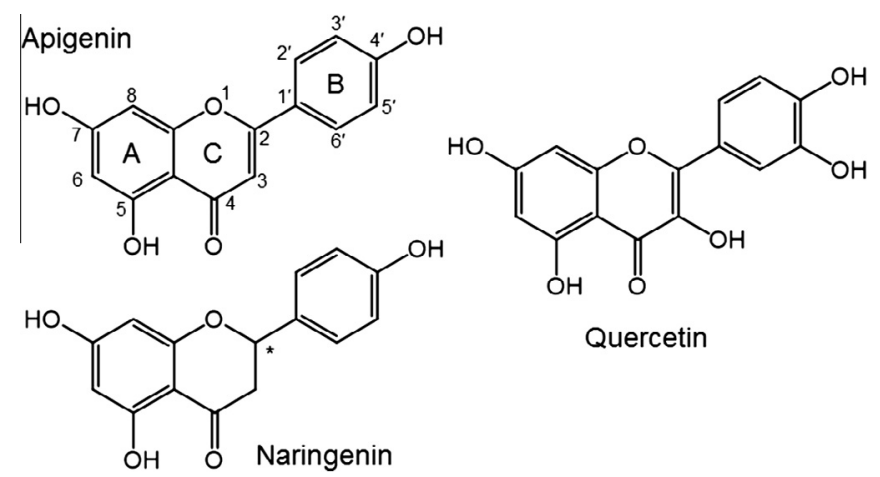

Figure 1. Structures of apigenin, naringenin and quercetin. Apigenin has strong XO inhibitor activity, while naringenin is practically inactive; the only difference between them is the presence or absence of the 2,3-double bond that allows flavones and flavonols to adopt a planar shape practically depending on the torsion of the $2,1^{\prime}$ bond only.

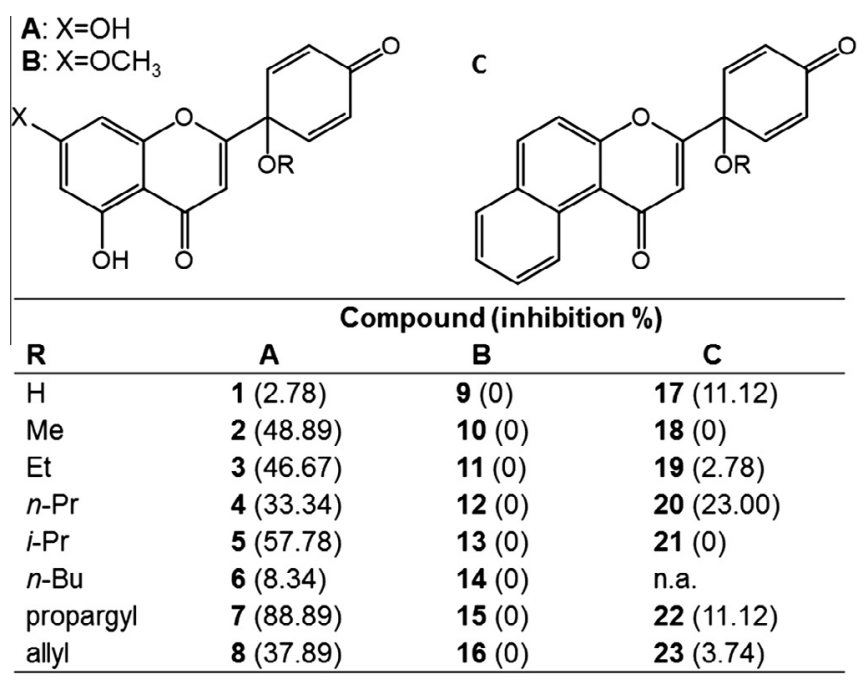

Figure 2. Structures of compounds 1-23 and their XO inhibition activities at $0.1 \mathrm{mM}$. A, B and C represent the corresponding basic skeleton. n.a.: not available. Apigenin, genkwanin and $4^{\prime}-\mathrm{OH}-\beta$-naphthoflavone, that is, the starting materials for the synthesis of these three groups of compounds, exhibited $98.3 \%, 33.3 \%$ and $26.0 \%$ inhibition at $0.1 \mathrm{mM}$, respectively, while allupurinol, used as positive control, inhibited the enzyme by $97.78 \%$ at $0.1 \mathrm{mM}$.

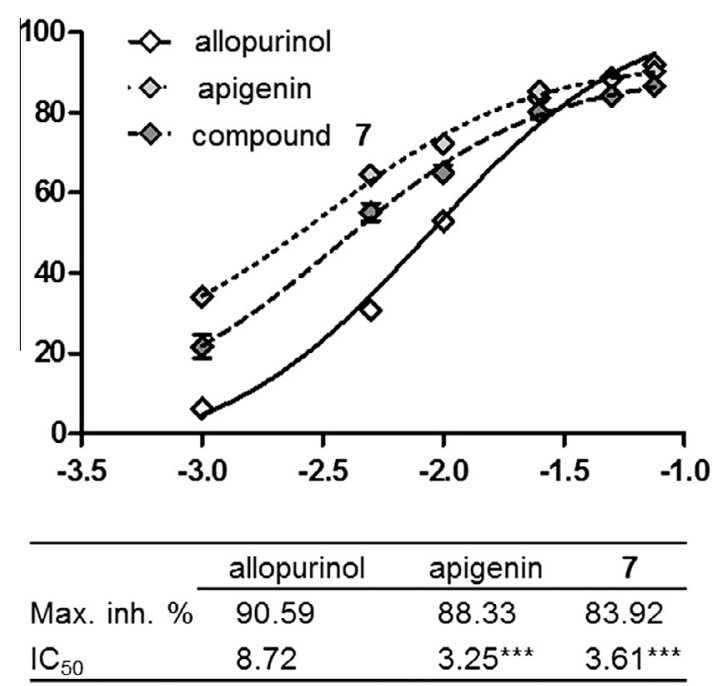

Figure 3. Dose-effect curves and $\mathrm{IC}_{50}$ values of compound $\mathbf{7}$, apigenin, and allopurinol. Max. inh.: maximum inhibition, ${ }^{* * *}$ : significant $(p<0.001)$ difference of the $\mathrm{IC}_{50}$ value with respect to that of allopurinol.

In this structure, quercetin is bound near the active center of the enzyme within the channel dedicated to the substrate. Since $\mathbf{7}$ is described here as a competitive inhibitor similar to quercetin, the $\mathrm{XO} / q u e r c e t i n$ complex appears as a relevant model for further docking, that is, replacing quercetin by $\mathbf{7}$ and using the same active site for the docking procedure. iGEMDOCK, a recently released docking and virtual screening tool utilizing the GEMDOCK scoring function was used, ${ }^{19,20}$ which conveniently combines an automatic preparation of the macromolecule around the bound ligands with docking and post-analysis. Re-docking the experimental ligand into the binding site resulted in a root mean square deviation (RMSD) value as low as 0.9149 A for the best-fit pose, which represented the most favorable van der Waals interactions. The results of this experiment and the best docking pose of $\mathbf{7}$, as well as its interactions with the enzyme are shown in Figure 5. 


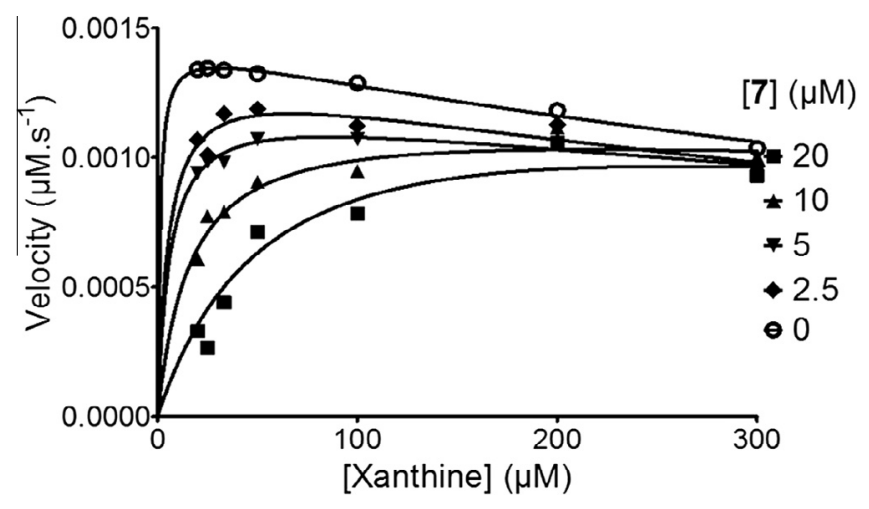

Figure 4. Enzyme kinetics study on the XO inhibition by compound 7. Curve-fitting was performed by using the substrate inhibition nonlinear model of GraphPad Prism 5.0; the concentrations of 7 are given in $\mu \mathrm{M}$.

Of the ten possible solutions found in the docking study of $\mathbf{7}$, the best four represented very similar positions, with binding energies of $-126.88,-126.33,-118.68$ and $-117.50 \mathrm{kcal} / \mathrm{mol}$, respectively. In the case of the fifth best solution, the $A$ and $C$ rings of 7 almost perfectly overlapped with those of quercetin within the X-ray structure, resulting in a somewhat less favorable binding energy $(-95.37 \mathrm{kcal} / \mathrm{mol})$. The remaining five solutions showed much weaker binding to $3 \mathrm{NVY}$ ( -68.11 to $-16.73 \mathrm{kcal} / \mathrm{mol}$ ).

In the most stable orientation of $\mathbf{7}$ in the XO active site, several H-bonds were observed (Fig. 5B), which differed from those existing in the presence of quercetin (Fig. 5A). This difference is mainly attributed to the flipped orientation of $\mathbf{7}$ with respect to that of quercetin, which modifies the H-bonding possibilities with residues of the active site. Interestingly, due to these flip-flop orientations, the 5-OH and 7-OH groups have inverted roles in quercetin and compound 7. Concerning quercetin, the $5-\mathrm{OH}$ group forms a $\mathrm{H}$-bond with the oxygen of the molybdopterin (MOS) residue, thus blocking its function. However, the 5-OH group of flavonoids is known to form a strong intramolecular $\mathrm{H}$-bond with the keto group at $\mathrm{C}-4,{ }^{21}$ which consequently weakens the intermolecular $\mathrm{H}$-bond with molybdopterin. In the case of compound $\mathbf{7}$, the 7-OH group forms this intermolecular $\mathrm{H}$-bond, which enhances effectively the binding to molybdopterin, and therefore the blocking of its function. This highlights the crucial role of the 7-OH group, fully rationalizing the dramatic loss of activity, for example, from $\mathbf{7}$ to $\mathbf{1 5}$ (Fig. 2).

The orientation of $\mathbf{7}$ in the XO active site is also stabilized by $\pi-\pi$ interactions between the $A$ and $C$ rings of the flavonoid and the aromatic ring of the Phe914 residue (Fig. 5B); these interactions are similarly observed for quercetin (Fig. 5A).

The most stable orientations found for $\mathbf{7}$ in the XO active site suggest a reasonable explanation for the importance of the propargyl side-chain: it fits perfectly into the hydrophobic pocket formed by the Leu648, Phe649, Asp872, Leu873 and His875 residues. Moreover, this orientation is also stabilized by a H-bond between the ether oxygen and Ser876. On the other hand, when the butyl ether derivative 6 was docked into 3NVY, its docking scores and positions could not explain its much lower activity compared to 7. Similar binding energies and orientations were found for these two compounds, and even the longer butyl ether side-chain was able to fit into the aforementioned pocket and form a H-bond with Ser876 at the same time. Side-chain length, on the other hand, might still be a limiting factor for these compounds to reach the active site through its relatively narrow access channel. This might explain the gradually decreasing XO inhibitor activity from the methyl to butyl derivatives (compounds 2-6) when tested at $0.1 \mathrm{mM}$.

Nevertheless, the previously detailed interactions anchor the inhibitor inside the channel through which the ligand can approach the molybdenum active center. Based on our results, the 'planar structure' criterion no longer appears as a major prerequisite for efficient XO inhibitor activity of flavonoid derivatives; unlike the case of flavanones, if loss of the $\pi$-conjugation from the B- to the Cring occurs at $\mathrm{C}-1^{\prime}$, the activity does not necessarily decrease as compared to flavones. Although the structure of protoapigenone $1^{\prime}$-O-propargyl ether certainly represents a rather special case among flavonoids, revision of this globally accepted structureactivity relationship might open novel directions for designing potent and safe $\mathrm{XO}$ inhibitors from this compound class. The planarity of the $\mathrm{A}$ and $\mathrm{C}$ moiety appears mandatory to favor intermolecular $\pi-\pi$ stacking, but flexibility is permitted for the moiety attached to C-2. For example, enhancing the binding to the Leu648-Phe649Asp872-Leu873-His875 hydrophobic pocket is a relevant strategy to improve selectivity of flavonoid-type XO-inhibitors.
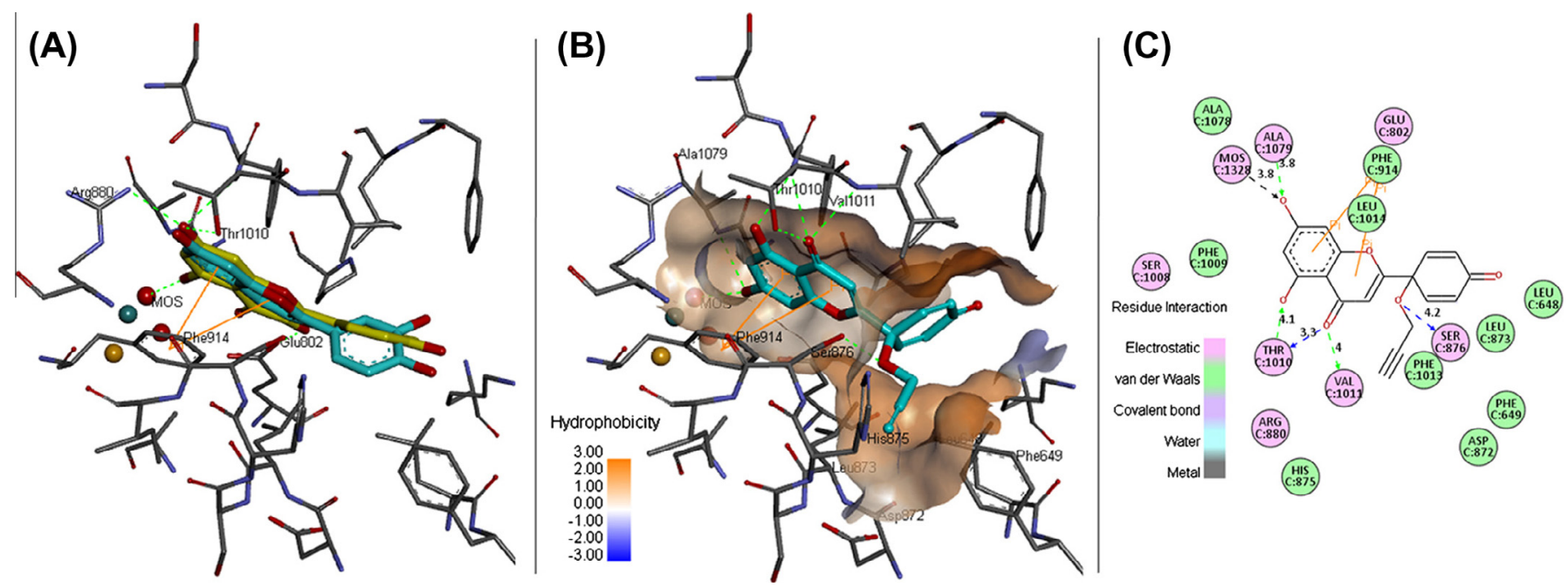

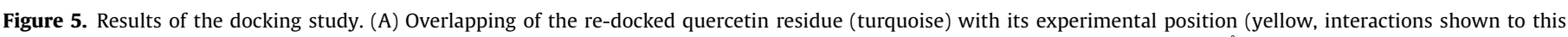

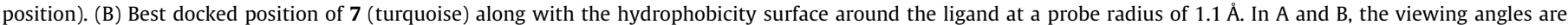

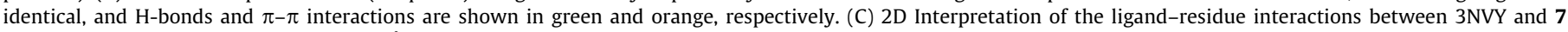
including the distances of the H-bonds in $\AA$. 


\section{Acknowledgments}

This work was supported by the European Union and co-funded by the European Social Fund (TAMOP-4.2.2/B-10/1-2010-0012, and TAMOP-4.2.2A-11/1/KONV-2012-0035), by the National Science Council, Executive Yuan, Taiwan (Wu YC), and partially by the Fundação para a Ciência e a Tecnologia, Portugal (PEsT-OE/SAU/ UI0074/2011), and it was performed within the framework of a bilateral mobility grant from the National Science Council, Taiwan and the Hungarian Academy of Sciences (102-2911-I-037-501 and SNK-79/2013). A.M. acknowledges the grant SFRH/BPD/81118/ 2011 provided by the Fundação para a Ciência e a Tecnologia, Portugal. B.D. was supported by an STSM Grant within the framework of COST Action CM0804, Chemical Biology with Natural Products. The authors thank the 'Conseil Régional du Limousin' for financial support and CALI (CAlcul en LImousin) for computing facilities, and gratefully acknowledge the support by the Operational Program Research and Development for Innovations-European Regional Development Fund (project CZ.1.05/2.1.00/03.0058) of the Ministry of Education, Youth and Sports of the Czech Republic.

\section{Supplementary data}

Supplementary data (experimental procedures) associated with this article can be found, in the online version, at http://dx.doi.org/ 10.1016/j.tetlet.2013.09.087.

\section{References and notes}

1. Terada, L. S.; Leff, J. A.; Repine, J. E. In Methods of Enzymology; Packer, L., Glazer, A. N., Eds.; Academic Press: New York, 1990; pp 651-656.
2. Higgins, P.; Dawson, J.; Walters, M. Cardiovasc. Psychiatry Neurol. 2009, 2009, 282059.

3. Pacher, P.; Nivorozhkin, A.; Szabó, C. Pharmacol. Rev. 2006, 58, 87-114.

4. Nepali, K.; Singh, G.; Turan, A.; Agarwal, A.; Sapra, S.; Kumar, R.; Banerjee, U. C.; Satti, N. K.; Gupta, M. K.; Suri, O. P.; Dhar, K. L. Bioorg. Med. Chem. 2011, 19, 1950-1958.

5. Nepali, K.; Agarwal, A.; Sapra, S.; Mittal, V.; Kumar, R.; Banerjee, U. C.; Satti, N. K.; Gupta, M. K.; Suri, O. P.; Dhar, K. L. Bioorg. Med. Chem. 2011, 19, 5569-5576.

6. Kumar, R.; Darpan; Sharma, S.; Singh, R. Expert Opin. Ther. Pat. 2011, 21, 1071 1108.

7. Avena-Woods, C.; Hilas, O. PET 2010, 35, 82-85.

8. Beiler, J. M.; Martin, G. J. J. Biol. Chem. 1951, 192, 831-834.

9. Zhu, J. X.; Wang, Y.; Kong, L. D.; Yang, C.; Zhang, X. J. Ethnopharmacol. 2004, 93, $133-140$.

10. Hayashi, T.; Sawa, K.; Kawasaki, M.; Arisawa, M.; Shimizu, M.; Morita, N. J. Nat. Prod. 1988, 51, 345-348.

11. Chang, W. S.; Lee, Y. J.; Lu, F. J.; Chiang, H. C. Anticancer Res. 1993, 13, 2165 2170.

12. Cos, P.; Ying, L.; Calomme, M.; Hu, J. P.; Cimanga, K.; Van Poel, B.; Pieters, L.; Vlietinck, A. J.; Berghe, D. V. J. Nat. Prod. 1998, 61, 71-76.

13. Nagao, A.; Seki, M.; Kobayashi, H. Biosci., Biotechnol., Biochem. 1999, 63, 1787 1790.

14. Van Hoorn, D. E. C.; Nijveldt, R. J.; Van Leeuwen, P. A. M.; Hofman, Z.; M'Rabet L.; De Bont, D. B. A.; Van Norren, K. Eur. J. Pharmacol. 2002, 451, 111-118.

15. Hunyadi, A.; Martins, A.; Danko, B.; Chang, F. R.; Wu, Y. C. Phytochem. Rev. 2013 http://dx.doi.org/10.1007/s11101-013-9288-2.

16. Hunyadi, A.; Chuang, D. W.; Danko, B.; Chiang, M. Y.; Lee, C. L.; Wang, H. C.; Wu, C. C.; Chang, F. R.; Wu, Y. C. PLoS ONE 2011, 6, e23922.

17. Danko, B.; Martins, A.; Chuang, D. W.; Wang, H. C.; Amaral, L.; Molnár, J.; Chang, F. R.; Wu, Y. C.; Hunyadi, A. Anticancer Res. 2012, 32, 2863-2870.

18. Rubbo, H.; Radi, R.; Prodanov, E. Biochim. Biophys. Acta 1991, 1074, 386-391.

19. Yang, J. M.; Chen, C. C. Proteins 2004, 55, 288-304.

20. Hsu, K. C.; Chen, Y. F.; Lin, S. R.; Yang, J. M. BMC Bioinformatics 2011, 12, S33.

21. Trouillas, P.; Marsal, P.; Lazzaroni, R.; Duroux, J. L. Food Chem. 2006, 97, 679688. 


\title{
Protoflavones: a class of unusual flavonoids as promising novel anticancer agents
}

\author{
A. Hunyadi $\cdot$ A. Martins $\cdot$ B. Danko $\cdot$ \\ F. R. Chang $\cdot$ Y. C. Wu
}

Received: 30 November 2012/ Accepted: 5 April 2013

(C) Springer Science+Business Media Dordrecht 2013

\begin{abstract}
Protoflavones represent a less widespread, unique class of natural flavonoids with a non-aromatic B-ring and a hydroxyl group at C-1' ${ }^{\prime}$. Due to their recently discovered anticancer activity, these compounds have gotten into the focus of biomedical research during the past few years. The present review aims to give a brief summary on the available literature data on this special class of flavonoids, including their occurrence in plants and their
\end{abstract}

\footnotetext{
A. Hunyadi $(\bowtie) \cdot$ B. Danko

Institute of Pharmacognosy, Faculty of Pharmacy,

University of Szeged, Szeged, Hungary

e-mail: hunyadi.a@pharm.u-szeged.hu
}

\section{A. Hunyadi}

COST Action CM0804 (Chemical Biology with Natural

Products) of the European Commission, Brussels,

Belgium

A. Martins

Department of Medical Microbiology and Immunobiology, Faculty of Medicine, University of Szeged, Szeged, Hungary

\author{
A. Martins \\ Unidade de Parasitologia e Microbiologia Médica, \\ Instituto de Higiene e Medicina Tropical, Universidade \\ Nova de Lisboa, Lisbon, Portugal \\ F. R. Chang \\ Graduate Institute of Natural Products, Kaohsiung \\ Medical University, Kaohsiung, Taiwan, ROC
}

bioactivity. A special emphasis is given on the anticancer potential of these compounds. Attempts for the development of certain synthetic/semi-synthetic protoflavone analogs as anticancer drugs, and structure-activity relationships are also discussed.

Keywords Anticancer flavonoids from ferns . Protoflavonoids · Protoapigenone $\cdot p$-Quinol · Natural product based drug discovery $\cdot$ Cancer $\cdot$ Apoptosis

\footnotetext{
F. R. Chang

Cancer Center, Kaohsiung Medical University Hospital, Kaohsiung, Taiwan, ROC

F. R. Chang

R\&D Center of Chinese Herbal Medicines and New

Drugs, College of Pharmacy, Kaohsiung Medical

University, Kaohsiung, Taiwan, ROC

Y. C. Wu ( $ه)$

School of Pharmacy, College of Pharmacy, China Medical University, 91, Hsueh-Shih Road, Taichung, Taiwan, ROC

e-mail: yachwu@mail.cmu.edu.tw

Y. C. Wu

Natural Medicinal Products Research Center, China Medical University Hospital, Taichung, Taiwan, ROC

Y. C. $\mathrm{Wu}$

Center for Molecular Medicine, China Medical University Hospital, Taichung, Taiwan, ROC
} 


\section{Introduction}

Flavonoids, polyphenolic compounds abundant in the Plant Kingdom, have long been recognized to play an important role in prevention and/or treatment of many pathological conditions (Di Carlo et al. 1999; Le Marchand 2002). Their chemical structure allows these compounds to participate in various redox reactions including free radical scavenging, while due to their typically phenolic characteristics they can also specifically interact with several biochemical processes on various levels (Tapas et al. 2008). The common belief, which considers flavonoids in general as beneficial and healthy-to-consume plant metabolites, is certainly based on a large body of scientific evidence. Most common members of this compound family are present in fruits and vegetables, and they even have been suggested to be vitamins (vitamin P, coming from "permeability") by the group of the Nobel-laureate Albert Szent-Györgyi (Bentsáth et al. 1936). Although they finally withdrew this theory, nowadays, more than 75 years later it is of no doubt that most typical dietary flavonoids have a generally positive impact on human health. One has to note, however, that several classes of flavonoids can exert very well defined, specific, sometimes strong bioactivities: isoflavones and prenylflavones are for example well known from their phytoestrogenic effect (Ososki and Kennelly 2003), and certain methoxylated flavones have been identified as potent antiviral agents (Hayashi et al. 1997). The present review focuses on the occurrence, chemistry and bioactivity of an unusual class of flavonoids, protoflavones, and aims to give a brief summary on the current trends in the research and development of protoflavone derivatives as potential anticancer agents.

\section{Protoflavones: chemistry and occurrence in nature}

Protoflavones are relatively oxidated flavonoids, bearing an unusual, non-aromatic B-ring with a hydroxyl group at $\mathrm{C}-1^{\prime}$. Although this nomenclature has not been explained, trivial names of protoflavones keep deriving from the corresponding $4^{\prime}$-hydroxyflavones in a way that a "proto-" prefix is added in case a $4^{\prime}$ hydroxy group is present (as in protogenkwanin), and an additional "-one" ending shows if it is oxidized to an oxo group (as in protogenkwanone). First member of this class of flavonoids, 2-(4- $\beta$-D-glucosyloxy-1hydroxy-2,5-cyclohexadienyl)-5-hydroxy-7-methoxychromone (14), was isolated from the fertile sprouts of Equisetum arvense and called protogenkwanin 4'glucoside (Hauteville et al. 1980). This was soon followed by reporting isolation of its aglycone, protogenkwanin (13), from the same source (Hauteville et al. 1981). The two hydroxyl groups of this compound on the B-ring at positions $1^{\prime}$ and $4^{\prime}$ were found to be trans oriented by means of X-ray crystallography (Stomberg et al. 1991), and similar orientation was found both for the partially (KlausPeter 1999) and the completely saturated B-ring containing protogenkwanin analogs (Wada et al. 1987). On the other hand, existence of the $1^{\prime}, 4^{\prime}$-cis form in the dihydroprotogenkwanin series was also argued by Pouny et al. (2011). Structures of the known natural protoflavones are shown in Fig. 1; occurrence of these compounds in plant species is summarized in Table 1.

As seen from the table, protoflavones are typical for certain genera of ferns, and, till now, Apium appears to be the only non-fern plant genus where such compounds were detected. Although biosynthesis of these compounds has not been investigated, a possible way for their production was suggested from the corresponding 4'-hydroxyflavone (i.e. apigenin or genkwanin) by oxidation. This step would then be followed by reduction of the 4 '-oxo group and/or saturation of either one or both double bond(s) in the already nonaromatic B-ring (Lin et al. 2007a). Interestingly, the formation of derivatives with further hydroxylation on a partially saturated B-ring seems to occur via a nonspecific way: $2^{\prime}$-hydroxy-2', $3^{\prime}$-dihydroprotoapigenone (3) was isolated from Equisetum fluviatile as a racemate (Pouny et al. 2011), and both enantiomers were also found to be present in Thelypteris torresiana (Fang et al. 2011; Liu et al. 2012). Such data on the enantiopurity of the related methoxy derivatives are, however, not available.

\section{Bioactivity of protoflavones}

In several cases, the isolation of new natural protoflavones was reported together with bioactivity data typically against various cancer cell lines. To date, however, protoapigenone (1) is far the most deeply studied among them. This compound and a number of 


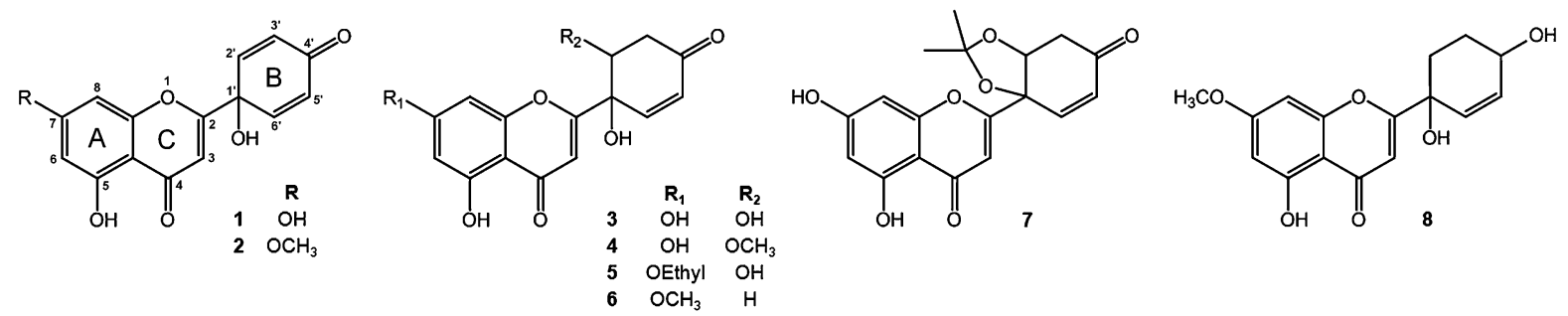
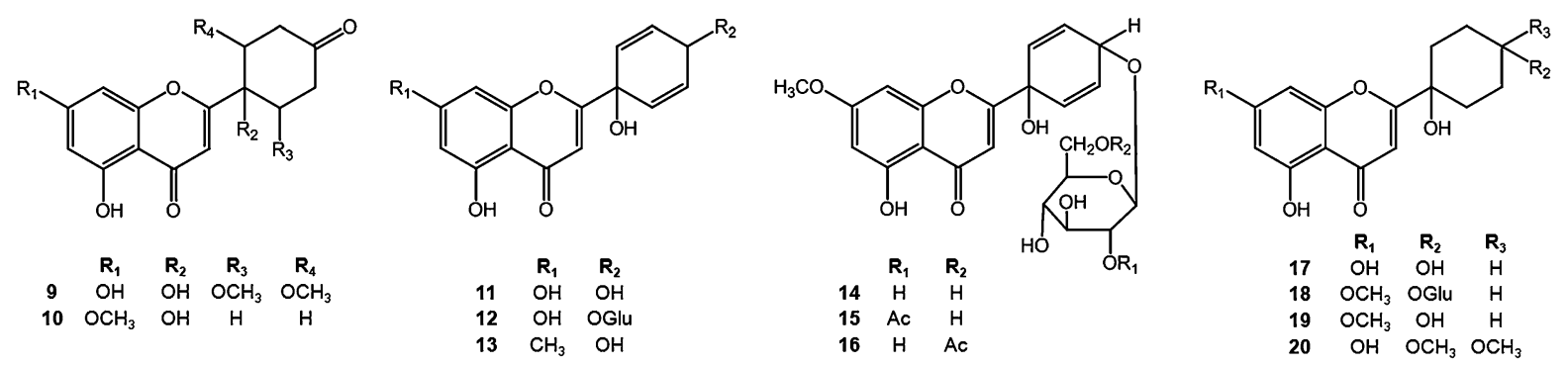

Fig. 1 Structures of natural protoflavones isolated from plants. Glu $\beta$-D-glucopyranoside. From 3, both the $(+)$ cis-1 $1^{\prime} R, 6^{\prime} S$ and the $(-) c i s-1^{\prime} S, 6^{\prime} R$ stereoisomers were isolated, as well as the racemate. Natural origin or artifact nature of $\mathbf{2 0}$ is unclear

its analogs were shown to exert strong cytotoxic activity in a wide variety of cancer cells including breast, ovarian, cervical, prostate, lung, liver, etc. (Lin et al. 2005, 2007a, b; Chang et al. 2008a, b; Chiu et al. 2009; Hunyadi et al. 2011; Danko et al. 2012). Chang et al. (2008a) also showed that protoapigenone (1) exerted strong cytotoxicity on ovarian cancer cell lines MDAH-2774 and SKOV3 but not on immortalized non-cancer ovarian epithelial cells. Moreover, several studies showed that this compound induces apoptosis both in vitro and in vivo, without significantly damaging healthy tissues in the latter case (Chang et al. 2008a, b; Wang et al. 2012). Cytotoxicity of protoapigenone (1) was recently also correlated with phenotypic changes in HeLa cells: impaired centrosomal $\gamma$-tubulin labeling was observed both in mitotic and interphasic cells (Pouny et al. 2011).

Concerning the mechanism of action, several pathways were found to be involved in the anticancer activity of protoflavones, and mainly of protoapigenone (1). Apoptosis induction was shown to be mitogen-activated protein kinase (MAPK)-dependent in both human prostate (Chang et al. 2008a) and breast cancer cells (Chen et al. 2011a), also involving the activation of c-Jun $\mathrm{NH}_{2}$-Terminal Kinase (JNK). Related apoptotic pathways were also identified using ovarian cancer cell lines (Chang et al. 2008b): activation of caspase-3, cleavage of PARP and decreasing of $\mathrm{Bcl}-\mathrm{xL}$ and $\mathrm{Bcl}-2$ protein levels were found, accompanied by membrane alterations and nuclear fragmentations at different stages of the cell cycle. Protoapigenone (1) was also shown to interfere with the cell cycle inducing its arrest at $\mathrm{S}$ and $\mathrm{G}_{2} / \mathrm{M}$ phases (Chang et al. 2008a, b; Chiu et al. 2009). Both in human ovarian (Chang et al. 2008a) and prostate (Chang et al. 2008b) cancer cells, the cell cycle arrest was correlated with alterations in the expression level of Cdk2, Cyclin B1 and Cdc25C, well known cell cycle regulators that actually lead to a higher cytotoxic effect of protoapigenone (1) at this stages of the cell cycle. In human breast cancer cells (Chen et al. 2011a) loss of the mitochondrial membrane potential was also observed and linked with the persistent activation of MAPK upon treatment with protoapigenone (1).

DNA damage in lung (Chiu et al. 2009) and prostate cancer cell lines (Chen et al. 2011b) treated by protoapigenone or one of its synthetic analogs (WYC0209, see section on synthetic/semi-synthetic derivatives) was also observed. Damage of DNA is an important and frequent outcome during cancer treatment. In fact, inhibition of DNA damage response mechanisms is an attracting novel target for enhancing tumor chemosensitivity (Lapenna and Giordano 2009; Bolderson et al. 2009). Remarkably, a recent study showed that protoapigenone (1) and WYC0209 can significantly interact with the ATR-dependent signaling pathways by inhibiting the phosphorylation of Chk2 and Chk1 kinases (Wang et al. 2012) that play a 
Table 1 Occurrence of protoflavones in plants

\begin{tabular}{|c|c|}
\hline Plant species & Protoflavones identified $^{\mathrm{a}}$ \\
\hline \multirow[t]{2}{*}{ Equisetum arvense } & Protogenkwanin $4^{\prime}-O-\beta$-D-glucoside (14) \\
\hline & Protogenkwanin (13) \\
\hline Pseudophegopteris hirtirachis & $\begin{array}{l}\text { Protogenkwanone (2); tetrahydroprotogenkwanone }(\mathbf{1 0}) \text {; } \\
\text { tetrahydroprotogenkwanin }(\mathbf{1 9}) \text {; protogenkwanin } 4^{\prime}-O-\beta-\mathrm{D}- \\
\text { glucoside }(\mathbf{1 4})\end{array}$ \\
\hline Pseudophegopteris subaurita & $\begin{array}{l}\text { Protogenkwanone }(\mathbf{2}) \text {; tetrahydroprotogenkwanone }(\mathbf{1 0}) \\
\text { protogenkwanin } 4^{\prime}-O \text { - } \beta \text {-D-glucoside }(\mathbf{1 4}) \text {; protogenkwanin } \\
4^{\prime}-O-(2-O \text {-acetyl)- } \beta \text {-D-glucoside }(\mathbf{1 5}) \text {; protogenkwanin } \\
4^{\prime}-O-(6-O \text {-acetyl)- } \beta \text {-D-glucoside }(\mathbf{1 6})\end{array}$ \\
\hline Pseudophegopteris bukoensis & $\begin{array}{l}\text { Protogenkwanin } 4^{\prime}-O-\beta \text {-D-glucoside }(\mathbf{1 4}) \text {; protogenkwanin } \\
4^{\prime}-O-(2-O \text {-acetyl })-\beta \text {-D-glucoside }(\mathbf{1 5}) \text {; protogenkwanin } \\
4^{\prime}-O-(6-O \text {-acetyl })-\beta \text {-D-glucoside }(\mathbf{1 6})\end{array}$ \\
\hline \multirow[t]{2}{*}{ Equisetum fluviatile } & Protoapigenin $4^{\prime}-O-\beta$-D-glucoside (12) \\
\hline & $\begin{array}{l}2^{\prime}, 3^{\prime} \text {-Dihydroprotogenkwanone }(\mathbf{6}) ;( \pm) 2^{\prime} \text {-hydroxy-2', } \\
3^{\prime} \text {-dihydroprotoapigenone }{ }^{\mathrm{b}}(\mathbf{3})\end{array}$ \\
\hline Equisetum debile & Protoapigenone (1) \\
\hline Cyclosorus falcilobus & Protoapigenin (11); protoapigenin $4^{\prime}-O$ - $\beta$-D-glucoside (12) \\
\hline Cyclosorus parasiticus & Protoapigenone (1) \\
\hline Phegopteris decursive-pinnata & $\begin{array}{l}\text { Protogenkwanone }(\mathbf{2}) ; 5^{\prime}, 6^{\prime} \text {-dihydroprotogenkwanone }(\mathbf{6}) \\
\text { negative for } \mathbf{1}\end{array}$ \\
\hline Phegopteris connectilis & $5^{\prime}, 6^{\prime}$-Dihydroprotogenkwanin $(\mathbf{8})$ \\
\hline \multirow[t]{6}{*}{$\begin{array}{l}\text { Thelypteris or Macrothelypteris } \\
\text { torresiana }\end{array}$} & $\begin{array}{l}\text { Protoapigenin (11); protoapigenin } 4^{\prime} \text { - } O \text { - } \beta \text {-D-glucoside }(\mathbf{1 2}) \\
\text { protoapigenone }(\mathbf{1}) ; 2^{\prime} \text {-methoxy- } 2^{\prime}, 3^{\prime} \text {-dihydroprotoapigenone }(\mathbf{4})\end{array}$ \\
\hline & Flavotorresin (20) \\
\hline & $2^{\prime}$-Hydroxy-2',3'-dihydroprotoapigenone acetonide (7) \\
\hline & $2^{\prime}, 6^{\prime}$-Dimethoxy-tetrahydroprotoapigenone (9) \\
\hline & $\begin{array}{l}\text { Tetrahydroprotoapigenine }(\mathbf{1 7}) ;(+) 2^{\prime} \text {-hydroxy-2', } \\
3^{\prime} \text {-dihydroprotoapigenone }(\mathbf{3})\end{array}$ \\
\hline & $(-) 2^{\prime}$-Hydroxy-2', $3^{\prime}$-dihydroprotoapigenone $(3)$ \\
\hline Macrothelypteris oligophlebia & Protoapigenone (1); protoapigenin $4^{\prime}-O-\beta$-D-glucoside (12) \\
\hline \multirow[t]{3}{*}{ Macrothelypteris viridifrons } & $\begin{array}{l}\text { Protoapigenone }(\mathbf{1}) \text {; protoapigenin }(\mathbf{1 1}) ; \text { protoapigenin } \\
4^{\prime} \text { - } O \text { - } \beta \text {-D-glucoside }(\mathbf{1 2}) ; 2^{\prime} \text {-hydroxy- } 2^{\prime}, 3^{\prime} \text {-dihydroprotoapigenone } \\
\text { acetonide }(\mathbf{7}) ; 2^{\prime}, 6^{\prime} \text {-dimethoxy-tetrahydroprotoapigenone }(\mathbf{9})\end{array}$ \\
\hline & 7-Ethoxy-2'-hydroxy-2',3'-dihydroprotoapigenone (5) \\
\hline & $\begin{array}{l}\text { Tetrahydroprotoapigenin (17); tetrahydroprotoapigenin } 4^{\prime}-O \text { - } \\
\beta \text {-D-glucoside }(\mathbf{1 8}) ; 2^{\prime} \text {-hydroxy- } 2^{\prime}, 3^{\prime} \text {-dihydroprotoapigenone (3); } \\
2^{\prime} \text {-methoxy-2', } 3^{\prime} \text {-dihydroprotoapigenone (4) }\end{array}$ \\
\hline Apium graveolens & Protoapigenin $4^{\prime}-O-\beta$-D-glucoside (12) \\
\hline
\end{tabular}

References

Hauteville et al. (1980)

Hauteville et al. (1981)

Wada et al. (1987)

Wada et al. (1987)

Wada et al. (1987)

Veit et al. (1995)

Pouny et al. (2011)

Pouny et al. (2011)

Pouny et al. (2011)

Pouny et al. (2011)

Pouny et al. (2011)

Klaus-Peter (1999)

Lin et al. (2005)

Lin et al. (2007a)

Tang et al. (2009)

Tang et al. (2010)

Fang et al. (2011)

Liu et al. (2012)

Wu et al. (2011)

Wei et al. (2011a)

Wei et al. (2011b)

Wei et al. (2012)

Nikolic et al. (2011)

\footnotetext{
${ }^{a}$ Several compounds listed in this table were reported with their IUPAC names; for a more clear interpretation here they are presented according to the classical nomenclature of protoflavones

b $\mathrm{OH}$ or $\mathrm{OCH}_{3}$ groups present at $\mathrm{C}-2^{\prime}$ in derivatives with partially saturated B-ring are cis to the $1^{\prime}$-OH group

${ }^{c}$ Depending on the botanical definition of Thelypteridaceae, the species can be assigned to different genera; both names were used in protoflavone related publications
} 
crucial role in DNA damage response (Smith et al. 2010). According to this, it was shown that these protoflavones themselves apparently do not directly damage DNA, the underlying mechanism is rather a specific inhibition of its repair. As expected, this inhibition could effectively sensitize the cancer cells to the DNA damaging anticancer drug cisplatin, resulting in significantly stronger combined activities as compared to those of the compounds administered alone (Wang et al. 2012). These results suggest that future development of protoflavones might reasonably be re-directed towards the optimization of this highly promising chemo-sensitizing activity, in order to obtain valuable new options for combination chemotherapy. Particular emphasis should be given to WYC0209, which was as much as 10 times stronger than protoapigenone (1) in some of the aforementioned activities.

Based on the chemical structure of the $p$-quinol B-ring, protoflavones seem to be capable for participating in various redox reactions. Indeed, apoptosis induced by protoapigenone (1) and other synthetic analogs were shown to be correlated with the induction of oxidative stress within the cancer cells by generating reactive oxygen species (ROS) (Chen et al. 2011a, b; Wei et al. 2011a, b; Liu et al. 2012). In connection to this, inhibition of the enzyme glutathione $S$-transferase $\pi$ was also observed, even though it is still to be determined if this was a direct effect on the enzyme or rather a result of the increase in ROS within the cell (Chen et al. 2011a). Studies on breast cancer cells showed that protoapigenone treatment led to increased levels of ROS, required for activation of MAPK involved in the mitochondria-mediated apoptosis induced by this compound (Chen et al. 2011a). Similar observations were made when other protoflavonoids, WYC0209 (Chen et al. 2011b), DHEC (Wei et al. 2011a, b) and DEDC (Liu et al. 2012) were tested for their apoptotic effect on DU145 postate cancer, human colon HT-29 cancer and human neuroblastoma SH-SY5Y cell lines, respectively. In fact, WYC0209 was shown to increase intracellular generation of ROS, followed by DNA damage induction and activation of Chk1/Chk2 which led to cell cycle arrest at $\mathrm{S}$ and G2/M phases (Chen et al. 2011b), and resulted in a stronger cytotoxic effect than that of protoapigenone (1) (Chang et al. 2008a). Due to the important role of ROS in the activity of protoflavones, it is currently hard to make a sound judgment on which of the previously mentioned pathways are subject to specific changes and which would be non-specifically affected by the oxidative stress; further research will be necessary to clarify such questions.

In addition to its anti-tumor activity, a very interesting anti-angiogenic effect was recently described for a protoflavone containing extract of Macrothelypteris viridifrons (see Table 1) (Wei et al. 2012). The same experiment was however not presented with the purified protoflavones-based on the crucial role of angiogenesis in tumor growth, biological significance of this important information should be explored in the near future.

Last but not the least, protoapigenone (1) was shown to inhibit the expression of Epstein-Barr virus (EBV) lytic proteins in P3HR1 cells, preventing EBV lytic proliferation at doses well below those which exerted cytotoxic activity on the host cells (Tung et al. 2011). This study also shows that the use of protoflavones is not limited to their anticancer activity; these unique flavonoids apparently represent a class of bioactive compounds with several further potential effects yet to be discovered.

\section{Development of synthetic/semi-synthetic protoflavone derivatives}

Based on its obvious structural relationship to apigenin, a well known natural chemopreventive agent (Patel et al. 2007), it is no wonder that the discovery of protoapigenone (1) and its promising anticancer activity (Lin et al. 2005) was rapidly followed by a raise of serious efforts for developing it as a new anticancer drug. First a total synthetic procedure was reported by our group, which, regardless of its limitations on the large-scale production of protoapigenone, allowed extending the known structure-activity relationships and led to the discovery of the strongest synthetic derivative till now, WYC0209 (Lin et al. 2007b). This compound, originated from 4 '-hydroxy- $\beta$-naphthoflavone, has become one of the most promising lead for further development. The structure of WYC0209, together with a selection of further prospective protoflavone analogs, is shown in Fig. 2.

After an optimization of the critical step of the total synthesis, namely the oxidative de-aromatization of the B-ring, it has been revealed that a semi-synthetic production of protoapigenone (1) is also possible 


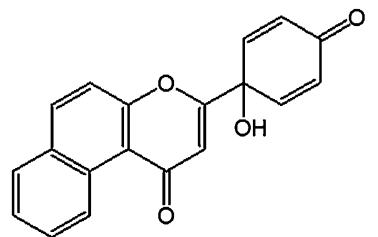

WYC0209

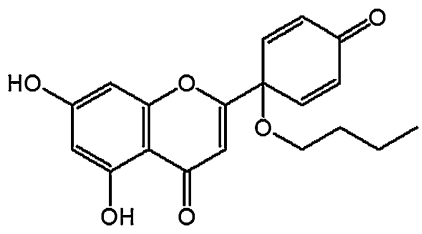

Protoapigenone 1'-O-butylether (21)

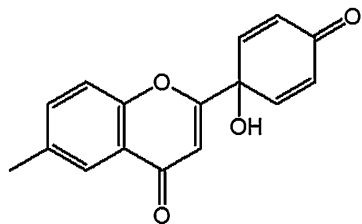

2-(1-Hydroxy-4-oxo-cyclohexa-2,5dienyl)-6-methyl-chromen-4-one (22)

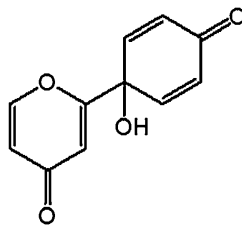

2-(1-Hydroxy-4-oxo-cyclohexa2,5-dienyl)-pyran-4-one (23)
Fig. 2 A selection of the synthetic/semi-synthetic protoflavone analogs which are the most promising for further development against cancer. WYC0209: the strongest pro-apoptotic protoflavone to date; 21: similar or stronger than protoapigenone with a much more stable B-ring; 22: stronger than protoapigenone with a slight MDR selectivity not found for other derivatives; 23: similar or stronger than protoapigenone possessing the smallest part of the pharmacophore to date directly from apigenin (Hunyadi et al. 2011). By allowing large-scale synthesis, this finding was a breakthrough for the in-depth pharmacological and toxicological investigation of protoapigenone (1) in vivo.

Considering current trends, there is also an effort to synthesize further promising derivatives with improved pharmacological properties at all possible levels. Based on the available data on structureactivity relationships, the presence of a symmetric and non-substituted $p$-quinol structure on the B-ring seems to be the most important moiety for a strong proapoptotic activity: none of the partly or completely saturated, nor the 4'-hydroxy compounds including their glycosides were found as potent in this regard as protoapigenone (1) (Lin et al. 2005, 2007b; Fang et al. 2011; Pouny et al. 2011; Wei et al. 2011a). Interestingly, the rule for the need of a free $1^{\prime}-\mathrm{OH}$ group is not general: although a methoxy group at C-1' definitely decreases cytotoxic activity, in case of protoapigenone analogs, a longer, non-branching aliphatic side-chain (n-propyl or $n$-butyl) can restore or even increase activity as compared to that of the parental compound (Hunyadi et al. 2011). This was however not true in case of any other analogs including similar derivatives of genkwanin (Hunyadi et al. 2011; Danko et al. 2012), where the only difference to the $1^{\prime}$ - $O$-alkylated protoapigenone series was the 7-methoxy group. Polarity differences might reasonably explain this phenomenon, but specific role of the 7-OH group can also not be excluded on the basis of the available data.

Slightly (around 1.5-fold) selective cytotoxicity of 6-methyl protoflavone derivatives on multi-drug resistant cancer cells as compared to the parental cell line was recently reported, which opened another prospective research direction (Danko et al. 2012). This finding seems to be of particular interest, since the ABCB1 efflux pump that conferred resistance to the tested cell line, is among the most prevalent MDR transporters (Sharom 2008), hence it represents a major reason for the common failure of chemotherapy. Exploring the SAR behind this selectivity is of particular importance, and has become a primary objective of our on-going research program. On the other hand, it is also noteworthy that none of the protoflavone derivatives synthesized from apigenin, $\beta$-naphthoflavone, genkwanin or 6-methoxyflavone were found to exert different cytotoxic activity on the MDR cell line as compared to its parental one (Danko et al. 2012). This means tumors can most likely not become resistant to protoflavones by over-expressing the aforementioned efflux pump.

It has also been revealed, that presence of the A-ring is not necessarily needed for a strong cytotoxic effect (Yuan et al. 2011), which supports well the original assumption that the pharmacophore of protoapigenone is the $p$-quinol moiety on the B-ring (Lin et al. 2005). The available structure-activity relationships concerning the cytotoxic activity of natural, semi-synthetic and synthetic protoflavone derivatives are summarized in Fig. 3.

\section{Conclusions and future prospects}

Protoflavones indeed represent a unique class of natural flavonoids, with a rather unexplained biosynthesis. The occurrence of racemic derivatives in plants is most unusual, and suggests the role of non-specific oxidation processes. This does not sound likely to be limited to certain plant species; investigation of further species biosynthesizing large amounts of $4^{\prime}$ - 


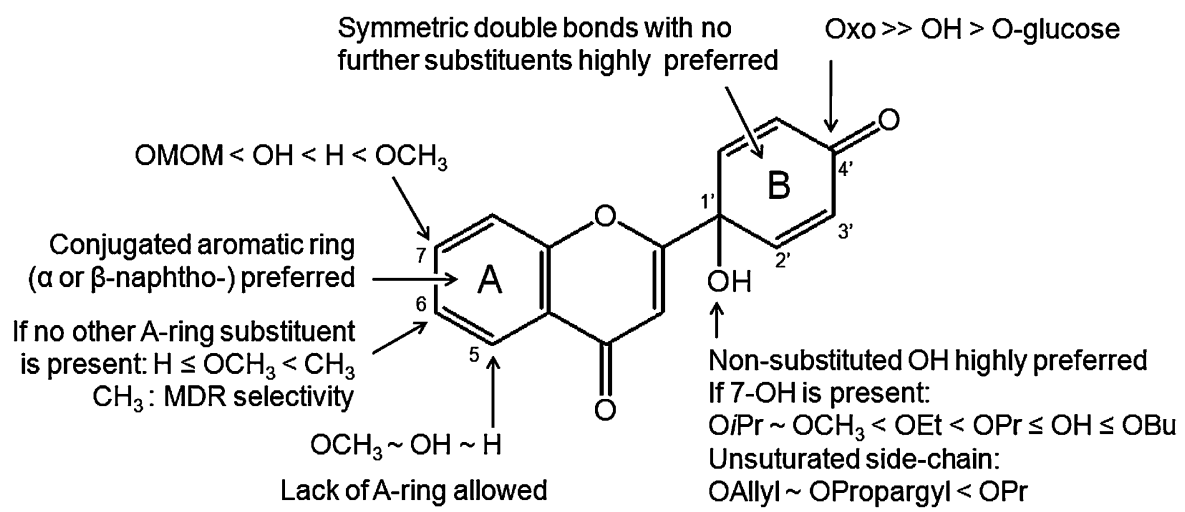

Fig. 3 A summary of SAR for the anticancer activity of natural, synthetic and semi-synthetic protoflavones. OMOM methoxymethyl, $i \operatorname{Pr}$ isopropyl, $\operatorname{Pr}$-propyl, Bu $n$-butyl

hydroxyflavones for presence of protoflavonoids would be particularly interesting in this point of view.

Above the aspects of basic research, protoflavones also represent a class of valuable bioactive compounds. First of all, on-going development of such compounds as anticancer drugs seems to be highly prospective; the emerging number of related patents indicates well the high expectations and optimism about this process (Wu et al. 2007, 2009; Lin et al. 2008; Yuan et al. 2010; Ruan et al. 2011). On the other hand, less cytotoxic protoflavone derivatives, including those with a partially or completely saturated B-ring, 4'-hydroxyl group containing compounds and most of the $1^{\prime}$-O-alkyl substituted ones, might have other interesting bioactivities worthy for further research. For example, some of the $1^{\prime}$ - $O$-alkylated compounds show stronger antiviral effects as compared to that of protoapigenone, certainly with a significantly lower cytotoxicity (unpublished data; publication in process). Based on this, one can presume that certain bioactivities of protoflavones are based on fundamentally different structure-activity relationships, which is going to extend research on the biological importance of these unique flavonoids and their derivatives in the near future.

Finally, some of these compounds seem to be on the right path to become classical examples for natural product based drug discovery. A number of semisynthetic or synthetic protoflavone derivatives are prospective leads for further development by utilizing the idea of a non-aromatic, $p$-quinol moiety in the B-ring discovered in the natural, fern-derived protoflavonoids. Protoapigenone (1) and WYC0209 are the first flavonoids described for their ability to inhibit
ATR related DNA damage response mechanisms both in vitro and in vivo, based on which these compounds or some of their analogs might have a real possibility to establish a novel class of anticancer drugs in the future.

Acknowledgments The authors acknowledge support of the European Union co-funded by the European Social Fund (TÁMOP 4.2.2/B-10/1-2010-0012 and TÁMOP-4.2.2.A-11/1/ KONV-2012-0035), the Fundação para a Ciência e para a Tecnologia, Portugal (PEsT-OE/SAU/UI0074/2011) and the grant from the National Science Council, Executive Yuan, Taiwan (Wu YC). Martins A is supported by the grant SFRH/ BPD/81118/2011, FCT, Portugal.

\section{References}

Bentsáth A, Rusznyák ST, Szent-Györgyi A (1936) Vitamin nature of flavones. Nature 138(7):798

Bolderson E, Richard DJ, Zhou BB, Khanna KK (2009) Recent advances in cancer therapy targeting proteins involved in DNA double-strand break repair. Clin Cancer Res 15:6314-6320

Chang HL, Su JH, Yeh YT, Lee YC, Chen HM, Wu YC, Yuan SSF (2008a) Protoapigenone, a novel flavonoid, inhibits ovarian cancer cell growth in vitro and in vivo. Cancer Lett 267:85-95

Chang HL, Wu YC, Su JH, Yeh YT, Yuan SSF (2008b) Protoapigenone, a novel flavonoid, induces apoptosis in human prostate cancer cells through activation of $\mathrm{p} 38$ mitogen-activated protein kinase and c-jun $\mathrm{NH} 2$-terminal kinase. J Pharmacol Exp Ther 325:841-849

Chen HM, Chang FR, Hsieh YC, Cheng YJ, Hsieh KC, Tsai LM, Lin AS, Wu YC, Yuan SS (2011a) A novel synthetic protoapigenone analogue, WYC02-9, induces DNA damage and apoptosis in DU145 prostate cancer cells through generation of reactive oxygen species. Free Rad Biol Med 50:1151-1162

Chen WY, Hsieh YA, Tsai CI, Kang YF, Chang FR, Wu YC, Wu CC (2011b) Protoapigenone, a natural derivative of 
apigenin, induces mitogen-activated protein kinasedependent apoptosis in human breast cancer cells associated with induction of oxidative stress and inhibition of glutathione S-transferase $\pi$. Invest New Drugs 29: 1347-1359

Chiu CC, Chang HW, Chuang DW, Chang FR, Cheng YS, Tsai MT, Chen WY, Lee SS, Wang CK, Chen JY, Wang HM, Chen CC, Liu YC, Wu YC (2009) Fern plant-derived protoapigenone leads to DNA damage, apoptosis, and $\mathrm{G}(2) / \mathrm{m}$ arrest in lung cancer cell line H1299. DNA Cell Biol 28(10):501-506

Danko B, Martins A, Chuang DW, Wang HC, Amaral L, Molnar J, Chang FR, Wu YC, Hunyadi A (2012) Cytotoxic activity of novel protoflavone analogs - selectivity towards a multidrug resistant cancer cell line. Anticancer Res 32(7):2863-2870

Di Carlo G, Mascolo N, Izzo AA, Capasso F (1999) Flavonoids: old and new aspects of a class of natural therapeutic drugs. Life Sci 65:337-353

Fang W, Ruan J, Cai Y, Wei A, Zhou D, Zhang W (2011) Flavonoids from the aerial parts of Macrothelypteris torresiana. Nat Prod Res 25(1):36-39

Hauteville M, Chopin J, Geiger H, Schueler L (1980) Protogenkwanin $4^{\prime}$-glucoside, a new type of natural flavonoid with a nonaromatic B-ring. Tetrahedron Lett 21(13): 1227-1230

Hauteville M, Chopin J, Geiger H, Schuler L (1981) Protogenkwanin, a new flavonoid from Equisetum arvense L. Tetrahedron 37(2):377-381

Hayashi K, Hayashi T, Otsuka K, Takeda Y (1997) Antiviral activity of 5,6,7-trimethoxyflavone and its potentiation of the antiherpes activity of acyclovir. J Antimicrob Chemother 39:821-824

Hunyadi A, Chuang DW, Danko B, Chiang MY, Lee CL, Wang HC, Wu CC, Chang FR, Wu YC (2011) Direct semi-synthesis of the anticancer lead-drug protoapigenone from apigenin, and synthesis of further new cytotoxic protoflavone derivatives. PLoS ONE 6(8):e23922

Klaus-Peter A (1999) Phenolic constitutents of the fern Phegopteris connectilis. Phytochemistry 52:929-934

Lapenna S, Giordano A (2009) Cell cycle kinases as therapeutic targets for cancer. Nat Rev Drug Discov 8:547-566

Le Marchand L (2002) Cancer preventive effects of flavonoids-a review. Biomed Pharmacother 56:296-301

Lin AS, Chang FR, Wu CC, Liaw CC, Wu YC (2005) New cytotoxic flavonoids from Thelypteris torresiana. Planta Med 71:867-870

Lin AS, Chang FR, Yen HF, Björkeborn HF, Norlén P, Wu YC (2007a) Novel flavonoids of Thelypteris torresiana. Chem Pharm Bull 55(4):635-637

Lin AS, Nakagawa-Goto K, Chang FR, Yu D, Morris-Natschke SL, Wu CC, Chen SL, Wu YC, Lee KH (2007b) First total synthesis of protoapigenone and its analogs as potent cytotoxic agents. J Med Chem 50(16):3921-3927

Lin AS, Wu YC, Lee KH, Chang FR (2008) Preparation of flavonoid compounds as anticancer agents. EP 1980248 A1 20081015

Liu H, Jiang C, Xiong C, Ruan J (2012) DEDC, a new flavonoid induces apoptosis via a ROS-dependent mechanism in human neuroblastoma SH-SY5Y cells. Toxicol In Vitro 26(1):16-23
Nikolic N, Cvetkovic D, Todorovic Z (2011) A characterization of content, composition and antioxidant capacity of phenolic compounds in celery roots. Ital J Food Sci 23: 214-219

Ososki AL, Kennelly EJ (2003) Phytoestrogens: a review of the present state of research. Phytother Res 17:845-869

Patel D, Shukla S, Gupta S (2007) Apigenin and cancer chemoprevention: progress, potential and promise (review). Int J Oncol 30:233-245

Pouny I, Etiévant C, Marcourt L, Huc-Dumas I, Batut M, Girard F, Wright M, Massiot G (2011) Protoflavonoids from ferns impair centrosomal integrity of tumor cells. Planta Med 77:461-466

Ruan J, Wei A, Xiong C, Wu G (2011) Method for preparing antitumor flavone-rich extract from Chinese medicine Thelypteris. CN 101966207 A 20110209

Sharom FJ (2008) ABC multidrug transporters: structure, function and role in chemoresistance. Pharmacogenomics 9(1):105-127

Smith J, Tho LM, Xu N, Gillespie DA (2010) The ATM-Chk2 and ATR-Chk1 pathways in DNA damage signaling and cancer. Adv Cancer Res 108:73-112

Stomberg R, Lundquist K, Hauteville M, Geiger H (1991) Crystal structure of protogenkwanin, $\mathrm{C}_{16} \mathrm{H}_{14} \mathrm{O}_{6}$. J Crystallogr Spectrosc Res 21(2):183-188

Tang Y, Fang W, Ma YT, Cai YL, Ruan JL (2009) A novel flavonoid from the root of Macrothelypteris torresiana (Gaud.) Ching. Chinese Chem Lett 20(7):815-816

Tang Y, Xiong C, Zhou D, Wei A, Fu W, Cai Y, Ruan J (2010) A new flavonoid from Macrothelypteris torresiana. Chem Nat Comp 46(2):209-211

Tapas AR, Sakarkar DM, Kakde RB (2008) Flavonoids as nutraceuticals: a review. Trop J Pharm Res 7(3):1089-1099

Tung CP, Chang FR, Wu YC, Chuang DW, Hunyadi A, Liu ST (2011) Inhibition of the Epstein-Barr virus lytic cycle by protoapigenone. J Gen Virol 92(Pt 8):1760-1768

Veit M, Bauer K, Beckert C, Kast B, Geiger H, Czygan FC (1995) Phenolic characters of british hybrid taxa in equisetum subgenus equisetum. Biochem Syst Ecol 23(1):79-87

Wada H, Fujita H, Murikami T, Saiki Y, Chen CM (1987) Chemical and chemotaxonomical studies of ferns. LXXIII.1) new flavonoids with modified B-ring from the genus Pseudophegopteris (Thelypteridaceae). Chem Pharm Bull 35(12):4757-4762

Wang HC, Lee AYL, Chou WC, Wu CC, Tseng CN, Liu KYT, Lin WL, Chang FR, Chuang DW, Hunyadi A, Wu YC (2012) Inhibition of ATR-dependent signaling by protoapigenone and its derivative sensitizes cancer cells to interstrand cross-link-generating agents in vitro and in vivo. Mol Cancer Ther 11:1443-1453

Wei A, Wu G, Xiong C, Zhou D, Cai Y, Ruan J (2011a) Flavonoids with special B-ring from Macrothelypteris viridifrons and their anti-proliferative effects on tumor cell. Zhongguo Zhong Yao Za Zhi 36(5):582-584

Wei A, Zhou D, Xiong C, Cai Y, Ruan J (2011b) A novel nonaromatic B-ring flavonoid: isolation, structure elucidation and its induction of apoptosis in human colon HT-29 tumor cell via the reactive oxygen species-mitochondrial dysfunction and MAPK activation. Food Chem Toxicol 49(9):2445-2452 
Wei A, Zhou D, Ruan J, Cai Y, Xiong C, Wu G (2012) Antitumor and anti-angiogenic effects of Macrothelypteris viridifrons and its constituents by HPLC-DAD/MS analysis. J Ethnopharmacol 139(2):373-380

Wu YC, Lin AS, Chang FR, Wu CC (2007) Flavonoids extracted from Pteridophyta for treating cancer cells, and preparation method for the same. US 20070104804 A1 20070510

Wu YC, Lin AS, Lee KH, Chang FR (2009) Composition comprising a flavonoid compound for treating cancer cells and synthetic method for the same. US 20090054516 A1 20090226

Wu G, Wei A, Cai Y, Xiong C, Ruan J (2011) Chemical constituents of Macrothelypteris oligophlebia and their antitumor activity in vitro and in vivo. Zhongguo Yaoxue Zazhi 46(5):330-333

Yuan SS, Wu YC, Chang HL Chang FR (2010) Pharmaceutical composition comprising protoapigenone for treating cancer and the signaling pathway thereof, and co-treatment with cisplatin. US 20100062078 A1 20100311

Yuan Q, Liu Z, Xiong C, Wu L, Wang J, Ruan J (2011) A novel, broad-spectrum antitumor compound containing the 1-hydroxycyclohexa-2,5-dien-4-one group: the disclosure of a new antitumor pharmacophore in protoapigenone 1 . Bioorg Med Chem Lett 21(11):3427-3430 


\title{
Lower antioxidative capacity of multidrug-resistant cancer cells confers collateral sensitivity to protoflavone derivatives
}

\author{
Tijana Stanković ${ }^{1}$ Balázs Dankó ${ }^{2} \cdot$ Ana Martins $^{3,4} \cdot$ Miodrag Dragoj $^{1}$ • \\ Sonja Stojkovic $^{1}$ - Aleksandra Isakovićc ${ }^{5}$ Hui-Chun Wang ${ }^{6}$ Yang-Chang Wu ${ }^{6,7,8,9}$. \\ Attila Hunyadi $^{2} \cdot$ Milica Pešić $^{1}$
}

Received: 29 April 2015 / Accepted: 7 July 2015

(C) Springer-Verlag Berlin Heidelberg 2015

\begin{abstract}
Purpose Multidrug resistance (MDR) may develop due to a series of adaptive responses under a new stress conditions, such as chemotherapy. Novel strategies are urgently needed to fight MDR in cancer, and chemotherapeutics that are selective for MDR cancer cells offer a promising approach. Certain protoflavones were previously found to have potential in this regard.

Methods Cytotoxicity of six protoflavones was assessed in different P-glycoprotein overexpressing MDR cancer cell lines and in their non-MDR counterparts. The impacts
\end{abstract}

Milica Pešić

camala@ibiss.bg.ac.rs; pesicmilica7@gmail.com

1 Institute for Biological Research, Department of Neurobiology, University of Belgrade, Bulevar Despota Stefana 142, 11060 Belgrade, Serbia

2 Institute of Pharmacognosy, University of Szeged, Eötvös u. 6, Szeged 6720, Hungary

3 Department of Medical Microbiology and Immunobiology, University of Szeged, Dóm tér 9, 6720 Szeged, Hungary

4 Unidade de Parasitologia e Microbiologia Médica, Institute of Hygiene and Tropical Medicine, Universidade Nova de Lisboa, Rua da Junqueira 100, 1349-008 Lisbon, Portugal

5 Faculty of Medicine, University of Belgrade, Doktora Subotića 8, Belgrade, Serbia

6 Graduate Institute of Natural Products, Kaohsiung Medical University, Shih-Chuan 1st Rd. 100, Kaohsiung 807, Taiwan

7 School of Pharmacy, College of Pharmacy, China Medical University, Taichung 404, Taiwan

8 Chinese Medicine Research and Development Center, China Medical University Hospital, Taichung 404, Taiwan

9 Center for Molecular Medicine, China Medical University Hospital, Taichung 404, Taiwan of compound 5, 6-methylprotoflavone previously published and a new derivative, 6-bromoprotoflavone (compound 6), on the cell cycle distribution were evaluated, and $\mathbf{6}$ was also studied for its potential to regulate the intracellular antioxidative capacity.

Results Protoflavones showed a significant cytotoxicity against all cancer cell lines and selectivity toward MDR cancer cells adapted to conventional chemotherapeutics. Inverse sensitivity versus MDR selectivity pattern was observed. Treatment with $\mathrm{H}_{2} \mathrm{O}_{2}$ showed that MDR cancer cells are more vulnerable to oxidative stress. Compounds 5 and $\mathbf{6}$ significantly decreased the portion of MDR cells in the G1 phase. The levels of reactive oxygen and nitrogen species (ROS/RNS) between MDR and non-MDR cells significantly differed upon exposure to $\mathbf{6}$, accompanied by changes in the glutathione (GSH) levels and in the expression of manganese superoxide dismutase (MnSOD), glutathione-S-transferase $\pi$ (GST $\pi$ ) and hypoxia-inducible factor- $1 \alpha$ (HIF-1 $\alpha$ ).

Conclusions Our results suggest that MDR cancer cells can be more vulnerable to the pro-oxidative activity of protoflavones due to an impaired antioxidative defense that might arise during the adaptation processes provoked by chemotherapy.

Keywords Multidrug resistance $\cdot$ P-glycoprotein · Protoflavones $\cdot$ Pro-oxidative activity $\cdot$ Reactive oxygen species $\cdot$ Collateral sensitivity

\section{Introduction}

Multidrug resistance (MDR) is the main reason for a non-successful chemotherapy in cancer. Still, chemotherapy cannot be excluded as a treatment option along with 
surgical intervention and/or radiotherapy. The capacity of cancer cells to develop resistance to chemotherapeutics is a significant challenge for new treatment strategies [1]. There is a wide range of cellular alterations acquired during the development of MDR: (1) overexpression of membrane efflux transporters belonging to the ATP-binding cassette family (e.g., the P-gp transporter, also known as P-glycoprotein or P-gp, which is the most prevalent among these so-called efflux pumps [2]), (2) extensively up-regulated DNA damage repair mechanisms, (3) impaired death signaling, (4) enhanced drug detoxification (e.g., up-regulation of glutathione-S-transferase enzymes), (5) lysosomal changes, (6) modification of drug molecular targets [3].

Collateral sensitivity (CS) could be explained as the ability of drug to be more efficient in MDR cancer cells than in their sensitive counterparts in the laboratory setting or as a phenomenon that may occur during the development of resistance when resistance to one or more drugs confers greater sensitivity to another agent [4]. The underlying mechanisms of CS may differ and may depend on the cell type and/or the applied drug. Recently proposed mechanisms that lead to this situation involve pro-oxidative effects and modification of intracellular redox status. According to available data, increased vulnerability to reactive oxygen species (ROS) could be the reason for hypersensitivity of certain MDR cells [5].

Compounds with a potential to overcome MDR by selective killing of such cancer cells have recently been reviewed [6]. Analogs of a flavonoid containing a nonaromatic A-ring, desmosdumotin $\mathrm{B}$, showed great promise against P-gp overexpressing MDR cell lines [7]. Moreover, several phenolic compounds, including xanthones and flavonoids, have been reported to exert selective cytotoxicity against ABCC1 (MRP1) overexpressing cancer cells typically due to their intrinsic pro-oxidant properties accompanied by an ability to promote glutathione (GSH) efflux through this ABC transporter [6].

Protoflavones have recently emerged as a class of natural compounds with prospective anticancer potential [8].
These unique flavonoids express an unusual non-aromatic B-ring; this p-quinol moiety makes them much stronger pro-oxidants than typical flavones. As such, these compounds can severely interfere with the redox homeostasis of cancer cells on multiple levels, leading to an oxidative stress-mediated apoptosis [9]. Based on protoapigenone (PA; compound 1), a natural protoflavone derived from apigenin, a set of prospective leads is under development including the synthetic $\beta$-naphthoflavone derivative WYC0209 (compound 2) [10]. According to our previous results, these compounds are unlikely to be P-gp substrates; moreover, 6-methyl derivatives showed a mild selectivity against a murine cancer cell line transfected with this efflux transporter [11].

Here we report the anticancer effects of six protoflavone derivatives (Fig. 1) on four MDR/non-MDR cancer cell line pairs: three pairs of human cancer $[12,13]$ and a pair of rat glioma cell lines [14], each developed by adaptation to various chemotherapeutics. Besides their cytotoxic activity, pro-oxidative effects of protoflavones as well as their impact on the antioxidative defense system of cancer cells were assessed.

\section{Materials and methods}

\section{Synthesis of protoflavones}

Compounds 1-5 were synthesized as published before [11, 15]. For compound 6, 5'-bromo-2'-hydroxyacetophenone was used as starting material. The flavonoid synthesis started with a Claisen-Schmidt condensation with benzyloxybenzaldehyde in a mixture of $\mathrm{EtOH}$ and aqueous solution of potassium hydroxide. The chalcone was cyclized with 1 equivalent (eq.) of iodine in DMSO, and the benzyl protecting group was removed with TFA in dichloromethane. The 6-bromo-4'-hydroxyflavone was dissolved in acetonitrile-water (9:1, v/v), and [bis(trifluoroacetoxy) iodo]benzene (PIFA; 2 eq.) was added to the mixture. The a

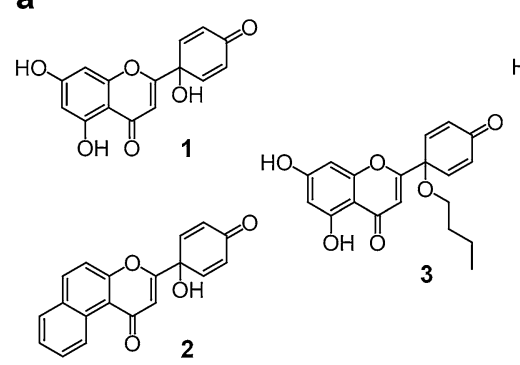

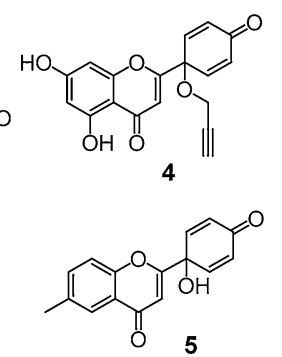

b

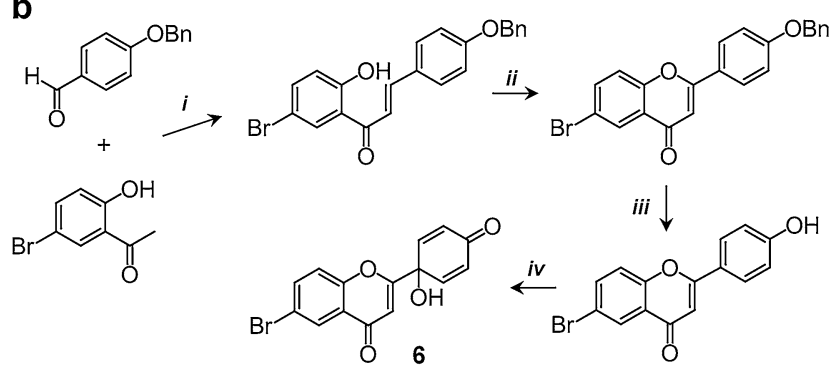

i. EtOH, 50\% KOH/ $\mathrm{H}_{2} \mathrm{O}$ (Yield 55\%); ii. $\mathrm{I}_{2}$ (1 eq)/DMSO, $110^{\circ} \mathrm{C}$ (Yield $84 \%$ ); iii. $\mathrm{CH}_{2} \mathrm{Cl}_{2} /$ TFA 3/1, reflux (Yield 32\%); iv. ACN/ $\mathrm{H}_{2} \mathrm{O} 9 / 1$, PIFA $80^{\circ} \mathrm{C}($ Yield $40 \%$ )

Fig. 1 Chemical structures of compounds 1-5 (a) and total synthesis of compound 6 (b) 
reaction mixture was heated to $50{ }^{\circ} \mathrm{C}$ for $1 \mathrm{~h}$ by using an oil bath. After the mixture was cooled down, the solvent was evaporated under reduced pressure, and the dry residue was re-dissolved in acetonitrile and purified by a Büchi flash chromatography system consisting of two C-606 pump and a C-640 ultraviolet detector (Büchi Labortechnik AG, Switzerland) on a prepacked RP C18 EC cartridge $(12 \times 150 \mathrm{~mm}, 40-63 \mu \mathrm{m}$, Büchi Labortechnik AG, Switzerland) with a gradient of aqueous $\mathrm{MeOH}(0-100 \%$ in $30 \mathrm{~min}$ ) at a flow rate of $8 \mathrm{ml} / \mathrm{min}$. For the chemical structures, see Fig. 1. All compounds were kept as $20 \mathrm{mM}$ stocks in dimethyl sulfoxide (DMSO) at $4{ }^{\circ} \mathrm{C}$ in dark prior to bioactivity testing.

\section{Structure elucidation of compound 6}

Structure elucidation was carried out by means of nuclear magnetic resonance (NMR) spectroscopy and high-resolution mass (HRMS) spectroscopy. NMR spectra were obtained on a Bruker Avance DRX-500 NMR spectrometer in dimethyl sulfoxide- $d 6$. HRMS spectra were taken on a Shimadzu LCMS-IT-TOF equipped with an ESI interface which was used in positive mode.

\section{Chemicals}

P-gp inhibitor-tariquidar (TQ) was kindly provided by Dr. Sven Rottenberg from the Netherlands Cancer Institute, Amsterdam. TQ was diluted in DMSO, and $10 \mu \mathrm{M}$ aliquots were kept at $-20{ }^{\circ} \mathrm{C}$. Before treatment, TQ and compounds 1-6 were freshly diluted in sterile water. RPMI 1640 medium, minimum essential medium (MEM), Dulbecco's modified Eagle's medium (DMEM), fetal bovine serum (FBS), antibiotic-antimycotic solution, penicillin-streptomicin solution, L-glutamine and trypsin/EDTA were purchased from PAA, Vienna, Austria. McCoy's 5 A medium, heat-inactivated horse serum and DMSO were obtained from Sigma-Aldrich Chemie Gmbh, Germany. Propidium iodide (PI) was purchased from Roche Applied Science, Basel, Switzerland. RNAse A and dihydrorhodamine 123 (DHR) were obtained from Molecular Probes ${ }^{\circledR}$, Invitrogen, USA.

\section{Cells and cell culture}

NCI-H460 (human non-small cell lung carcinomaNSCLC), DLD1 (human colorectal carcinoma), U87 (human glioma), C6 (rat glioma) and MRC-5 (human embryonal bronchial epithelial) cell lines were purchased from the American Type Culture Collection, Rockville, MD. HaCaT cell line (normal human keratinocytes) was obtained from CLS-Cell Lines Service, Eppelheim, Germany. NCI-H460/R cells were selected originally from
NCI-H460 cells after 3 months of doxorubicin selective pressure [12]. DLD1-TxR and U87-TxR cells were selected by continuous exposure to stepwise increasing concentrations of paclitaxel (PTX) for a period of 10 and 9 months from DLD1 and U87 cells, respectively [13]. RC6 cells were selected from $\mathrm{C} 6$ cells after continuous exposure to stepwise increasing concentrations of carmustine (10$300 \mu \mathrm{M}$ ) for a period of 9 months [14]. MDR cancer cell lines (NCI-H460/R and DLD1-TxR) and their sensitive counterparts were maintained in RPMI 1640 medium supplemented with $10 \%$ FBS, $2 \mathrm{mM}$ L-glutamine and 10,000 $\mathrm{U} / \mathrm{ml}$ penicillin, $10 \mathrm{mg} / \mathrm{ml}$ streptomycin, $25 \mu \mathrm{g} / \mathrm{ml}$ amphotericin B solution, while U87 and U87-TxR were grown in MEM with $10 \%$ FBS, L-glutamine $(2 \mathrm{mM})$ and $5000 \mathrm{U} /$ $\mathrm{ml}$ penicillin, $5 \mathrm{mg} / \mathrm{ml}$ streptomycin solution at $37{ }^{\circ} \mathrm{C}$ in a humidified $5 \% \mathrm{CO}_{2}$ atmosphere. C6, RC6, HaCaT and MRC-5 cells were cultured in DMEM supplemented with $10 \%$ FBS, $2 \mathrm{mM}$ L-glutamine, $4.5 \mathrm{~g} / \mathrm{l}$ glucose, $5000 \mathrm{U} / \mathrm{ml}$ penicillin and $5 \mathrm{mg} / \mathrm{ml}$ streptomycin solution at $37{ }^{\circ} \mathrm{C}$ in a humidified $5 \% \mathrm{CO}_{2}$ atmosphere. All adherent cell lines were subcultured at $72 \mathrm{~h}$ intervals using $0.25 \%$ trypsin/ EDTA and seeded into a fresh medium at the following densities: 8000 cells $/ \mathrm{cm}^{2}$ for NCI-H460, DLD1, DLD1TxR, C6 and RC6; 16,000 cells $/ \mathrm{cm}^{2}$ for U87 and NCI$\mathrm{H} 460 / \mathrm{R}$; and 32,000 cells/ $\mathrm{cm}^{2}$ for U87-TxR.

\section{Cell growth inhibition by MTT assay}

Cell viability was assessed by MTT assay (MTT, Sigma, St. Louis, MO, USA). Briefly, 4000 cells/well were seeded overnight in 96-well microplates and the following day, treated with protoflavones $(0.05-25 \mu \mathrm{M})$ or $\mathrm{H}_{2} \mathrm{O}_{2}$ (0.1-2.5 mM), by replacing the medium of each well with drug-containing fresh media and incubated for 72 and $48 \mathrm{~h}$, respectively. At the end of the incubation period, MTT was added to each well in a final concentration of $0.2 \mathrm{mg} /$ $\mathrm{ml}$ for $4 \mathrm{~h}$. Formazan product was extracted from cells by DMSO, and the absorbance was measured at $540 \mathrm{~nm}$ using an automatic microplate reader (LKB 5060-006 Micro Plate Reader, Vienna, Austria). $\mathrm{IC}_{50}$ value was defined as a concentration of each drug that inhibited cell growth by $50 \%$, and it was calculated by linear regression analysis using Excel software.

\section{Flow cytometric analysis of cell cycle distribution}

NCI-H460, NCI-H460/R, U87 and U87-TxR cells were plated in $25 \mathrm{~cm}^{2}$ flasks and incubated overnight. The effects of compounds 5 and $\mathbf{6}$ on cell cycle distribution were studied after $48 \mathrm{~h}$. Briefly, the attached and floating cells were trypsinized and collected by centrifugation, washed in phosphate buffer saline (PBS) and fixed in $70 \%$ ethanol for $48 \mathrm{~h}$ at $-20{ }^{\circ} \mathrm{C}$. After fixation, the cells were washed in 
PBS and pretreated with $133 \mu \mathrm{g} / \mathrm{ml}$ of RNAse A at $37{ }^{\circ} \mathrm{C}$ for $15 \mathrm{~min}$. Then, PI was added to final concentration of $17 \mu \mathrm{g} / \mathrm{ml}$. After $30 \mathrm{~min}$ of incubation, flow cytometric analysis was performed on the FACSCalibur flow cytometer (Becton-Dickinson, Oxford, UK). A minimum of 10,000 events was collected and analyzed for each experimental sample. Cell cycle distribution was determined automatically in Mod-FIT (Verity Software House, Inc.). The validity of the data analysis model was verified using the RCS value (reduced Chi-square, RCS $<15 \%$ ).

\section{RNA extraction and reverse transcription (RT) reaction}

Total RNA was isolated from NCI-H460, NCI-H460/R, U87 and U87-TxR cells. The isolation was carried out using TRIzol ${ }^{\circledR}$ reagent (Invitrogen Life Technologies, USA) according to the manufacturer's instructions. RNA was quantified by spectrophotometry, and quality was determined by agarose gel electrophoresis. RT reactions were performed using $2 \mu \mathrm{g}$ of total RNA, with a highcapacity cDNA reverse transcription kit (Applied Biosystems, USA), following the manufacturer's instructions.

\section{Real-time quantitative PCR}

To determine GST $\pi$, MnSOD and HIF- $1 \alpha$ mRNA expression level in NCI-H460, NCI-H460/R, U87 and U87-TxR cells, real-time PCR was performed using specific primers [16-18]. Prepared cDNAs were amplified using Maxima SYBR Green/ROX qPCR Master Mix (Thermo Scientific, USA), according to the recommendations of the manufacturer, in a total volume of $20 \mu 1$ in an ABI PRISM 7000 Sequence Detection System (Applied Biosystems, USA). Thermocycler conditions comprised of an initial step at $50{ }^{\circ} \mathrm{C}$ for $5 \mathrm{~min}$, followed by a step at $95{ }^{\circ} \mathrm{C}$ for $10 \mathrm{~min}$ and a subsequent 2-step PCR program at $95{ }^{\circ} \mathrm{C}$ for $15 \mathrm{~s}$ and $60{ }^{\circ} \mathrm{C}$ for $60 \mathrm{~s}$ for 40 cycles. The accumulation of PCR products was detected in real time, and the results were analyzed using the 7000 System Software (Applied Biosystems, USA) and presented as $2-\Delta \mathrm{Ct}$ [19], where $\Delta \mathrm{Ct}$ is the difference between $\mathrm{Ct}$ values of specific genes and the endogenous control ( $\beta$-actin) [20].

\section{Colorimetric detection of GSH}

U87 and U87-TxR cells grown in $25 \mathrm{~cm}^{2}$ flasks were trypsinized and counted. The same number of untreated or treated cells $\left(1 \times 10^{6}\right)$ was used for this experiment. Cells were pelleted by centrifugation and washed with ice-cold PBS. After that, cells were lysed in $80 \mu \mathrm{l}$ ice-cold glutathione buffer (ApoGSHTM Glutathione Colorimetric Detection Kit, BioVision Inc, USA) for $10 \mathrm{~min}$ on ice. Then, $20 \mu$ of $5 \%$ sulfosalicylic acid was added and the samples were centrifuged for $10 \mathrm{~min}$ at $4{ }^{\circ} \mathrm{C}$. The supernatant was transferred to a fresh tube and used for GSH assay. For the detection of GSH, glutathione buffer was added to each well (96-well plate) at a volume of $160 \mu$ l. Afterward, $20 \mu \mathrm{l}$ of either prepared samples or standards was added. Finally, $20 \mu \mathrm{l}$ of substrate solution was added and the absorbance of generated product (2-nitro-5-thiobenzoic acid) was read at $405 \mathrm{~nm}$ (LKB 5060-006 Microplate Reader, Austria). The concentrations of GSH $(\mu \mathrm{g} / \mu \mathrm{l})$ were determined using the standard calibration curve. The detection was performed at least four times in three independent experiments.

\section{Reactive oxygen/nitrogen species (ROS/RNS) detection}

Flow cytometric analysis of dihydrorhodamine 123 (DHR) fluorescence intensity was performed to detect ROS/RNS levels (peroxynitrite anion formation through the reaction of the two free radicals superoxide and nitric oxide) in U87 and U87-TxR cells untreated and treated with $400 \mathrm{nM}$ 5 or $4 \mu \mathrm{M} \mathrm{6}$. Levels of hydrogen peroxide and peroxynitrite anion were assessed. U87 and U87-TxR cells were incubated overnight in adherent 6-well plates $(100,000$ cells/well). Then, cells were treated with protoflavones for $72 \mathrm{~h}$. Cells were harvested by trypsinization and incubated in PBS with or without the P-gp inhibitor tariquidar (TQ) ranging concentration from 0.5 to $10 \mathrm{nM}$, in order to exclude the impact of P-gp transporter activity on DHR accumulation [21]. After $5 \mathrm{~min}, 1 \mu \mathrm{M}$ DHR was added for another $30 \mathrm{~min}$ at $37{ }^{\circ} \mathrm{C}$ in the dark. Cells were subsequently washed twice in ice-cold PBS. DHR fluorescence was analyzed by flow cytometry in the FL1-H channel. A minimum of 10,000 events was assayed for each sample. The samples were analyzed on a FACSCalibur (BectonDickinson, UK) flow cytometer.

\section{Statistical analysis}

Statistical analysis was performed by Statistica 6.0 and GraphPad Prism 6 Software. The differences between groups were tested by Student's $t$ test and were considered statistically significant if $p<0.05$. Main effects of protoflavones in different cell lines were examined by two-way ANOVA and analyzed by multiple comparisons.

\section{Results}

\section{Chemical properties of new 6-bromoprotoflavone}

A new protoflavone derivative, 6-bromoprotoflavone (6), was obtained by using a four-step total synthesis. The structure elucidation of this compound was straightforward: 
Table 1 Sensitivity of non-MDR and MDR cancer cells and normal human cells to six protoflavone derivatives and $\mathrm{H}_{2} \mathrm{O}_{2}\left(\right.$ expressed as $\mathrm{IC}_{50}$ values in $\mu \mathrm{M}$ )

\begin{tabular}{llllllcc}
\hline & 1 & 2 & 3 & 4 & 5 & 6 & $\mathrm{H}_{2} \mathrm{O}_{2}$ \\
\hline NCI-H460 & 0.83 & 0.10 & 0.92 & 2.89 & 0.22 & 8.94 \\
NCI-H460/R & $0.48^{*}$ & 0.07 & 0.90 & $1.05^{* *}$ & $0.08^{* *}$ & $3.85^{* *}$ & $136^{*}$ \\
DLD1 & 0.45 & 0.06 & 0.49 & 0.71 & 0.12 & 1.87 \\
DLD1-TxR & 0.39 & 0.04 & 0.41 & 0.56 & 0.09 & $0.91^{* *}$ & $312^{*}$ \\
U87 & 2.05 & 0.41 & 2.47 & 2.66 & 0.77 & 7.94 \\
U87-TxR & $0.91^{* *}$ & 0.34 & $1.38^{*}$ & $1.64^{*}$ & $0.35^{* *}$ & $4.13^{* *}$ \\
C6 & 2.50 & 0.21 & 4.64 & 2.50 & 0.37 & $1000^{*}$ \\
RC6 & $5.93^{\# \#}$ & $0.76^{\# \#}$ & $9.01^{\#}$ & $11.79^{\# \#}$ & $1.30^{\# \#}$ & 3.35 \\
HaCaT & 0.22 & $3.99^{\$ \$}$ & 3.21 & 0.47 & $1.92^{\$ \$}$ & $10.00^{\# \#}$ \\
MRC-5 & 0.28 & $3.05^{\$ \$}$ & 2.17 & 1.00 & 0.48 & 9.41 & $189^{\#}$ \\
\hline
\end{tabular}

n.d. not determined

* Statistically significant sensitivity of MDR cancer cells: * $p<0.05 ; * * p<0.01$

\# Statistically significant resistance of MDR cancer cells: ${ }^{\#} p<0.05 ;{ }^{\# \#} p<0.01 ;{ }^{\# \#} p<0.001$

$\$$ Statistically significant resistance of normal cells: ${ }^{\$} p<0.05 ; \$ \$ p<0.01$

HRMS confirmed the expected molecular formula of $\mathrm{C}_{15} \mathrm{H}_{10} \mathrm{BrO}_{4}$, and appearance of the characteristic p-quinol moiety was evidenced from the ${ }^{1} \mathrm{H}$ NMR spectrum, while the A-ring signals and the $\mathrm{H}-3$ singlet of the parental 6-bromo-4'-hydroxyflavone were retained. Experimental details of the new protoflavone derivative are as follows.

6-Bromoprotoflavone (6). Yellow, amorphous solid; HRMS: $\mathrm{C}_{15} \mathrm{H}_{10} \mathrm{BrO}_{4}$, calculated: 332.9762, found: 332.9763; ${ }^{1} \mathrm{H}$ NMR $(500 \mathrm{MHz}$, DMSO-d6) $\delta 8.08(1 \mathrm{H}, \mathrm{d}$, $J=2.35 \mathrm{~Hz}, \mathrm{H}-5), 7.91(1 \mathrm{H}, \mathrm{dd}, J=8.97,2.40 \mathrm{~Hz}, \mathrm{H}-7)$, $7.41(1 \mathrm{H}, \mathrm{d}, J=8.70 \mathrm{~Hz}, \mathrm{H}-8), 7.02(2 \mathrm{H}, \mathrm{d}, J=10 \mathrm{~Hz}$, $\mathrm{H}-2^{\prime}$ and $\left.\mathrm{H}-6^{\prime}\right), 6.66(1 \mathrm{H}, \mathrm{s}, \mathrm{H}-3), 6.35(2 \mathrm{H}, \mathrm{d}, J=9.95 \mathrm{~Hz}$, $\mathrm{H}-3^{\prime}$ and $\mathrm{H}-5^{\prime}$ ); ${ }^{13} \mathrm{C}$ NMR (500 MHz, DMSO- $d 6$ ) $\delta 185.39$ (C-4'), 174.12 (C-4), 167.77 (C-2), 146.09 (C-9), 140.85 (C-2', C-6'), 134.39 (C-7), 128.88 (C-5), 124.46 (C-3', C-5'), 120.02 (C-10), 118.39 (C-6), 112.68 (C-8), 107.79 (C-3), $68.28\left(\mathrm{C}-1^{\prime}\right)$.

The structures of the previously known compounds 1-5 as well as the synthesis of the new protoflavone $\mathbf{6}$ are presented in Fig. 1.

\section{Cytotoxic effects of protoflavone derivatives}

The effect of protoflavone derivatives on cell growth was evaluated by the MTT assay. The results are summarized in Table 1 . The efficacy and selectivity of protoflavones varied between different cell lines. Sensitivity of NCI-H460/R and U87-TxR to protoflavones was significantly higher in comparison with their sensitive counterparts (NCI-H460 and U87, respectively). However, although the strongest cytotoxic activity for all tested compounds was observed on the DLD1 and DLD1-TxR cells, with $\mathrm{IC}_{50}$ values at nanomolar levels, only $\mathbf{6}$ showed a significant selectivity toward
DLD1-TxR. Compounds with a substituent at C6 such as 5 and $\mathbf{6}$ showed higher selectivity toward the three human MDR cancer cell lines than $\mathbf{2}$, and the sensitivity of all tested cancer cell lines to $\mathbf{5}$ was greater than to $\mathbf{6}$. In contrast with the three human cancer cell line pairs, rat MDR glioma cells RC6, which developed a strong antioxidative defense system during the course of resistance induction, were more resistant to all tested protoflavones than their corresponding sensitive C6 cells (Table 1). This fact prompted us to investigate the sensitivity of employed cells to $\mathrm{H}_{2} \mathrm{O}_{2}$, an inducer of intracellular oxidative stress. When compared to their sensitive counterparts, human MDR cancer cells were more susceptible to the effects of $\mathrm{H}_{2} \mathrm{O}_{2}$, while rat MDR glioma cells were more resistant (Table 1). Normal human cells were significantly less sensitive to $\mathbf{2}$, while sensitivity to $\mathbf{5}$ and $\mathbf{6}$ decreased in $\mathrm{HaCaT}$ (normal keratinocytes) and MRC-5 (embryonal bronchial epithelial) cells, respectively (Table 1). The selectivity of $\mathbf{5}$ and 6 toward human glioma cells was examined next to human normal cells (Fig. 2a). Efficacy of $\mathbf{5}$ was significantly lowered in $\mathrm{HaCaT}$, while efficacy of $\mathbf{6}$ was significantly lowered in MRC-5 in comparison with both non-MDR and MDR glioma cells (Fig. 2a).

\section{Analysis of cell cycle distribution after protoflavones treatments}

Next, we examined whether the influence of compounds 5 and $\mathbf{6}$ on the cell cycle is correlated with their selectivity toward NCI-H460/R and U87-TxR cells. The differences between cell cycle distribution on non-MDR (NCI-H460 and U87) and MDR (NCI-H460/R and U87-TxR) cancer cells after $48 \mathrm{~h}$ treatment with $\mathbf{5}$ and $\mathbf{6}$ were assessed 

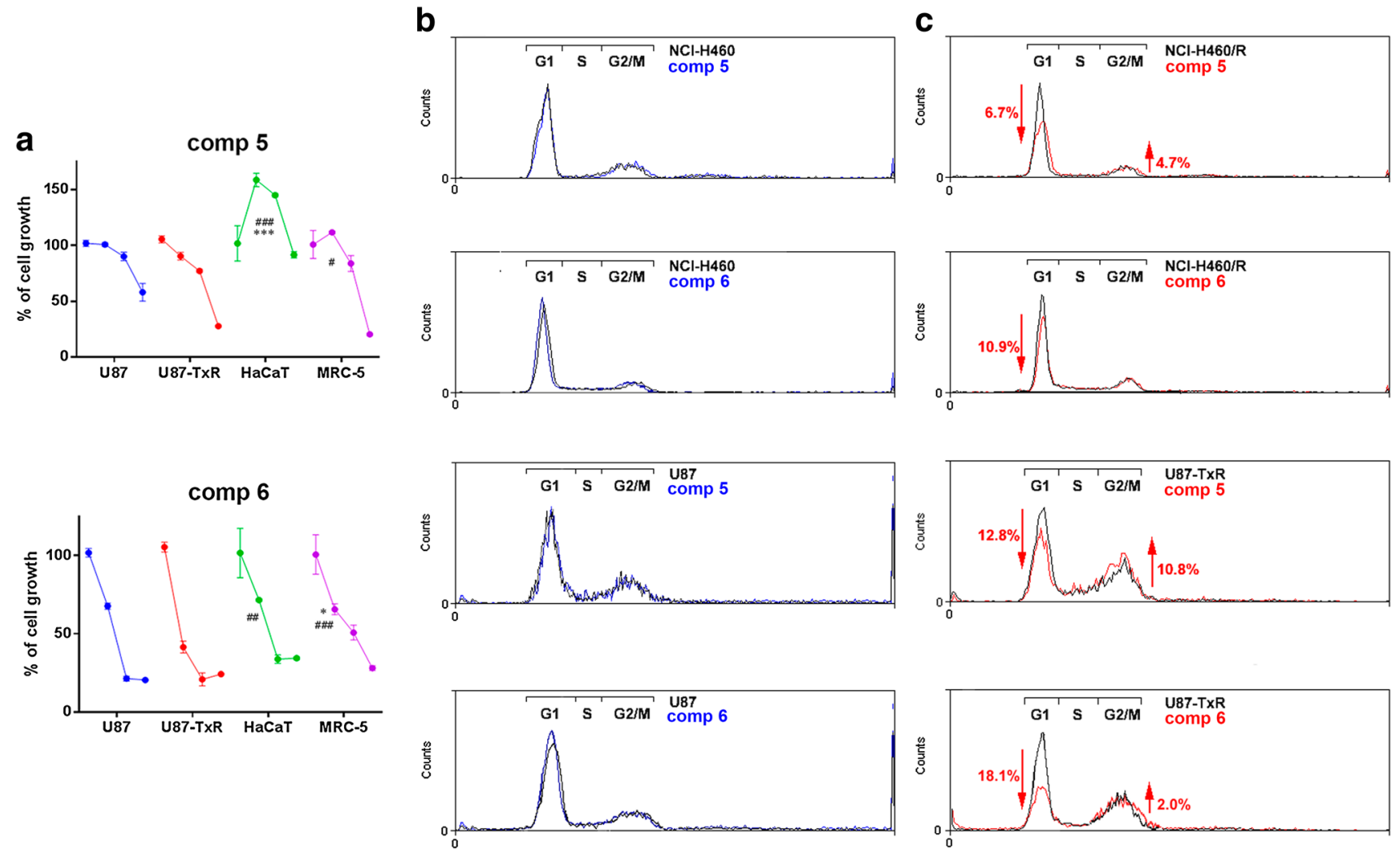

Fig. 2 Selectivity toward normal human cells and changes in the cell cycle distribution in human NSCLC and glioma cell lines upon protoflavone treatment. Comparison of protoflavones efficacy in glioma and normal cells assessed by MTT (a). Presented concentrations for $5(0.1,0.25$ and $0.5 \mu \mathrm{M})$ and $6(5,10$ and $25 \mu \mathrm{M})$. Statistical difference in main effect toward non-MDR (*) and MDR (\#) glioma cells. Unsynchronized non-MDR (b) and MDR cancer (c) cells were incubated in complete medium and treated with 5 or $\mathbf{6}$ for 48 h. NCI-

by flow cytometry (Fig. 2). $\mathrm{IC}_{50}$ concentrations of 5 and 6 on MDR cells were used in this experiment. Decrease in $\mathrm{G} 1$ phase and increase in $\mathrm{G} 2 / \mathrm{M}$ phase were observed after treatment with compound $\mathbf{5}$ in the MDR cancer cells (Fig. 2c), while non-MDR cells remained unaffected (Fig. 2b). Decrease in G1 phase caused by compound 6 was even greater than that of $\mathbf{5}$, while cell cycle arrest in $\mathrm{G} 2 / \mathbf{M}$ was not obvious (Fig. 2c). Non-MDR cells did not respond to treatment with 6 (Fig. 2b) at the employed concentration with this experimental setup.

\section{Antioxidative capacity of MDR and non-MDR cells}

The antioxidative capacity of NCI-H460, NCI-H460/R, U87 and U87-TxR was evaluated by comparing the sensitivity to $\mathrm{H}_{2} \mathrm{O}_{2}$ and the expression of antioxidative enzymes (Fig. 3). Sensitivity profiles for $\mathrm{H}_{2} \mathrm{O}_{2}$ differed between NCI-H460 and NCI-H460/R cells (Fig. 3a) as well as between U87 and U87-TxR cells (Fig. 3b). Moreover,
H460 and NCI-H460/R cells were treated with $100 \mathrm{nM}$ of 5 or $4 \mu \mathrm{M}$ of $\mathbf{6}$, while U87 and U87-TxR cells were treated with $400 \mathrm{nM}$ of 5 or $4 \mu \mathrm{M}$ of $\mathbf{6}$. Cell cycle analysis was performed by FACSCalibur flow cytometry, using Mod-FIT software. $Y$-axis: relative cell numbers. $X$-axis: DNA content by propidium iodide. The percents of cells in $\mathrm{G} 1$ and $\mathrm{G} 2 / \mathrm{M}$ represent average change between untreated and treated samples calculated from three repeated experiments

glioma cells (U87 and U87-TxR) showed a considerable resistance to $\mathrm{H}_{2} \mathrm{O}_{2}$ treatment reaching $\mathrm{IC}_{50}$ values in the millimolar range (Table 1; Fig. 3b). Expression of enzymes involved in the antioxidative defense and oxidative stress, GST $\pi$, MnSOD and HIF- $1 \alpha$ was evaluated by real-time qPCR (Fig. 3c, d). The expression of MnSOD and HIF- $1 \alpha$ mRNA was twofold and fourfold lower in NCIH460/R than in NCI-H460 cells, respectively (Fig. 3c). The expression of GST $\pi$ mRNA did not change significantly in NCI-H460/R cells (Fig. 3c), while in U87-TxR cells, it decreased threefold in comparison with the non-MDR U87 cells (Fig. 3d). HIF-1 $\alpha$ mRNA expression also significantly decreased in U87-TxR cells, while MnSOD level increased threefold compared to U87 (Fig. 3d).

\section{Bioactivity of compounds 5 and 6}

Effects of $\mathbf{5}$ and $\mathbf{6}$ on the GSH levels and on the ROS/RNS production in U87 and U87-TxR cells (Fig. 4a-d) were also 


\section{c}

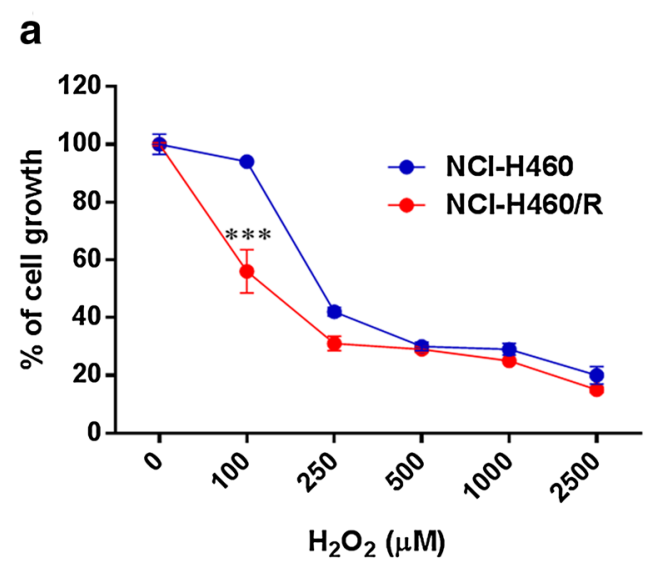

b

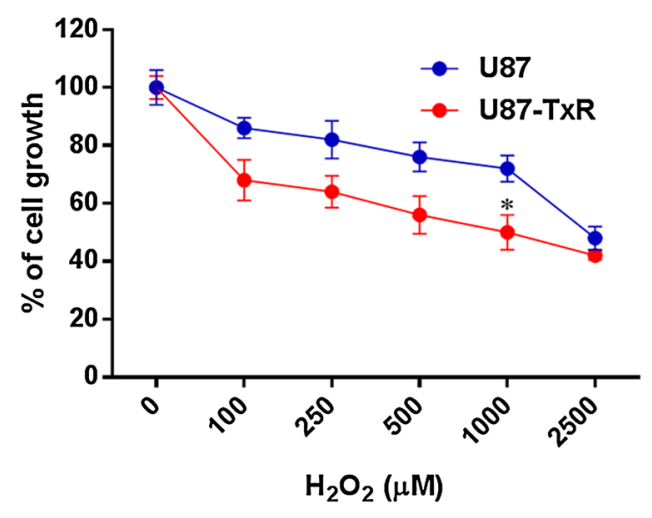

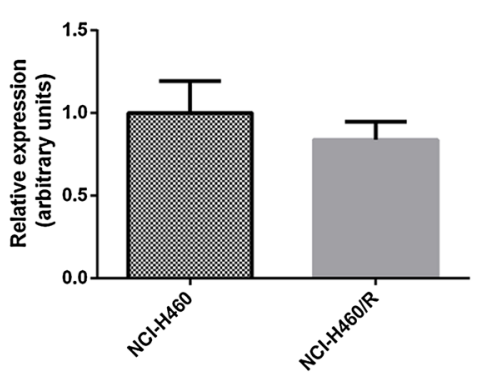

MnSOD

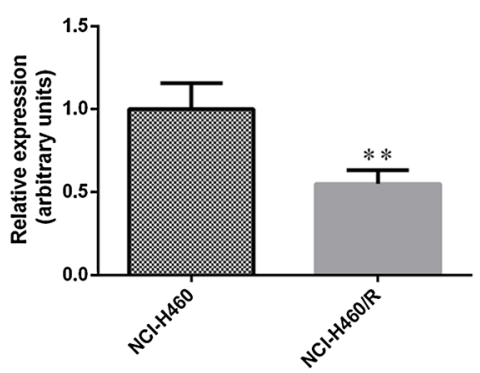

HIF-1 $\alpha$

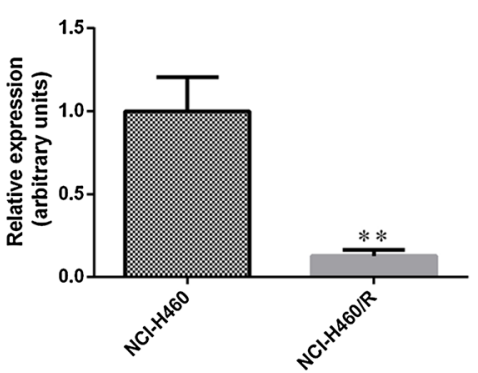

d
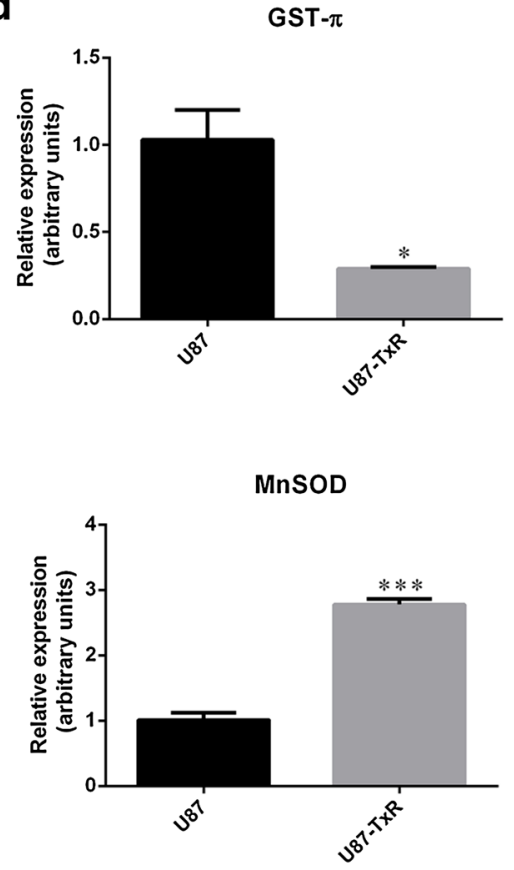

HIF-1 $\alpha$

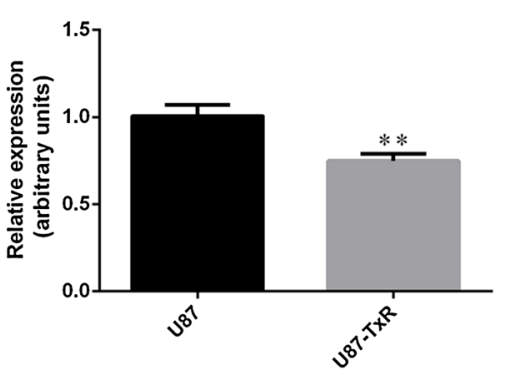

Fig. 3 Comparison of antioxidative capacity between non-MDR and MDR cancer cells. Cell growth inhibition assessed by MTT assay after $48 \mathrm{~h} \mathrm{H}_{2} \mathrm{O}_{2}$ treatment $(\mathbf{a}, \mathbf{b})$. The average $\pm \mathrm{SD}$ was obtained from four independent experiments $(n=4)$. Statistical significance between treated samples of NSCLC (a) and glioma cells (b):
$* p<0.05 ; * * * p<0.001$. qRT-PCR analysis of GST $\pi$, MnSOD and HIF- $1 \alpha$ mRNA expression in NSCLC (c) and glioma cells (d). Statistical significance between non-MDR and MDR cells from three independent experiments $(n=3)$ : * $p<0.05 ; * * p<0.01$; *** $p<0.001$ evaluated. Both compounds significantly elevated the level of GSH in U87 and U87-TxR cells (Fig. 4a). Because DHR (a dye for ROS/RNS detection) is a substrate of the P-gp transporter, its accumulation was evaluated in the presence of the P-gp inhibitor TQ (Fig. 4b). As expected, the difference between the DHR accumulation within non-MDR and MDR cells decreased upon TQ treatment. Maximum pump inhibition in U87-TxR cells was achieved with $5 \mathrm{nM}$ TQ (Fig. 4b). Therefore, basal ROS/RNS production in U87 and U87-TxR cells was considered when P-gp pump was inhibited by $5 \mathrm{nM}$ TQ. Then, the effect of compounds 5 and 6 on ROS/RNS production was studied in the presence of 5 nM TQ (Fig. 4c, d). No detectable change in the ROS/
RNS production was observed in the U87 cells upon treatment with 5 or 6 (Fig. 4c), while both compounds significantly increased it in U87-TxR cells (Fig. 4d). According to the obtained results, compounds $\mathbf{5}$ and $\mathbf{6}$ share the same potential for the engagement of GSH detoxification system and ROS/RNS production. Therefore, only the mechanism of the new protoflavone derivative, $\mathbf{6}$, on the mRNA expression levels of antioxidant enzymes was investigated. Compound 6 significantly decreased the expression of GST $\pi$ and HIF- $1 \alpha$ by twofold in U87 cells (Fig. 4e). MnSOD expression was decreased by twofold in U87-TxR cells, while HIF-1 $\alpha$ was negligibly detected after treatment of this cell line with compound 6 (Fig. 4e). 


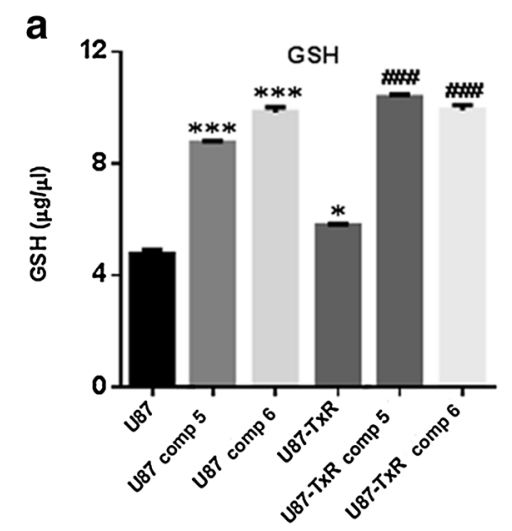

C
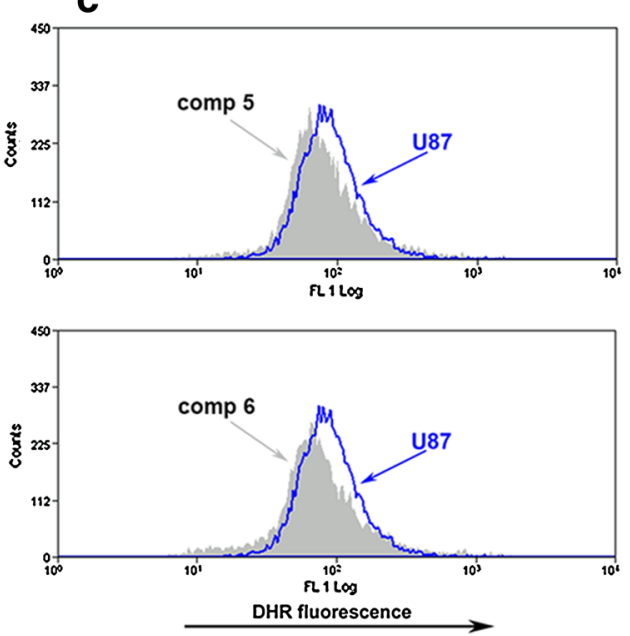

b

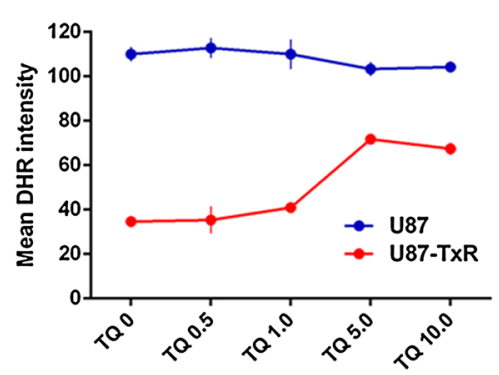

d
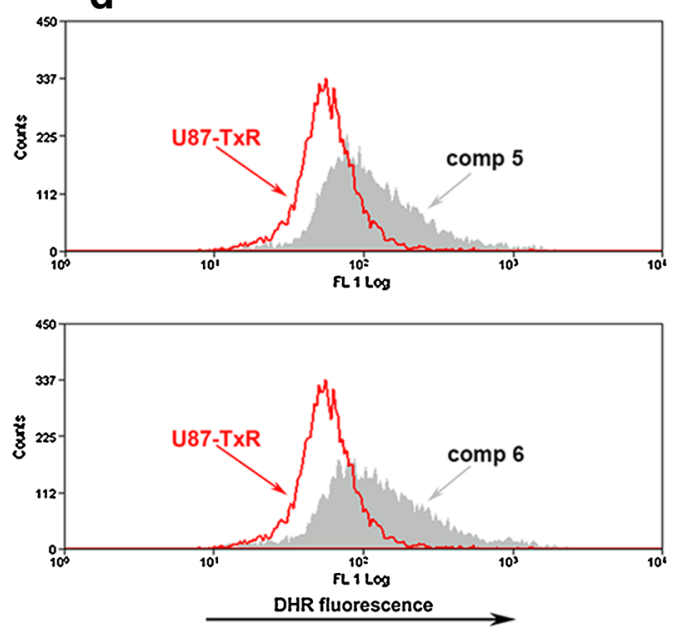

e
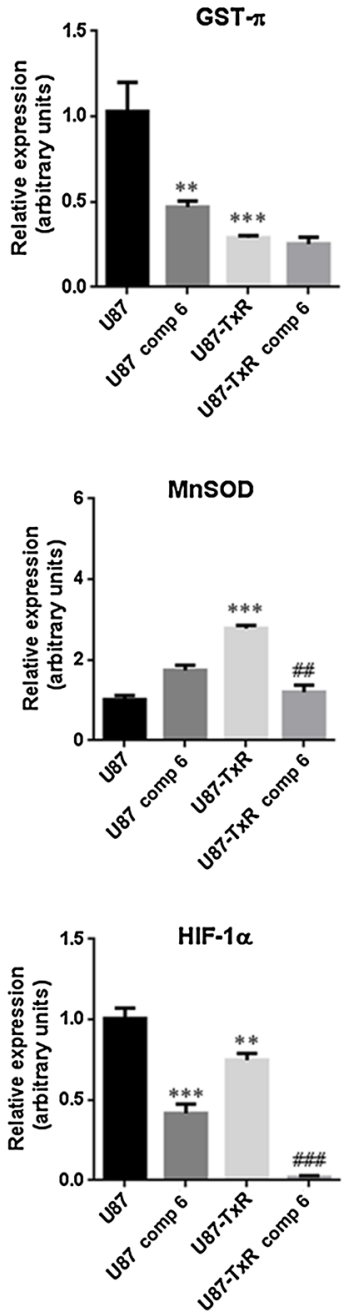

Fig. 4 Impact of protoflavone derivatives on intracellular antioxidative defense systems in non-MDR and MDR glioma cells. Levels of GSH in U87 and U87-TxR cells after treatment with 5 or $\mathbf{6}$ (a). Comparison of mean DHR intensity (as an indicator of ROS/RNS production) in U87 and U87-TxR cells treated with P-gp inhibitor TQ (b). Changes in DHR fluorescence (ROS/RNS production) after treatment with $400 \mathrm{nM}$ of $\mathbf{5}$ or $4 \mu \mathrm{M}$ of $\mathbf{6}$ in U87 (c) and U87-TxR cells

\section{Discussion}

The potential of certain protoflavone derivatives as MDR selective anticancer agents [11] encouraged us to test their activity in a panel of cancer cell lines with different levels of resistance, from different origins. Notably, a much stronger collateral sensitivity toward protoflavones was found in human MDR cancer cell lines than observed previously in P-gp-transfected mouse T cell lymphoma cells [11]. Although human MDR cancer cell lines utilized in this study displayed the P-gp overexpression similar to P-gp-transfected mouse $\mathrm{T}$ cell lymphoma cells, a major difference is that MDR phenotype in these cell lines was (d). qRT-PCR analysis of changes in GST $\pi$, MnSOD and HIF- $1 \alpha$ mRNA expression after treatment with $4 \mu \mathrm{M}$ of 6 (e). Statistical significance in respect to non-MDR glioma cells (U87) from three independent experiments $(n=3)$ : ** $p<0.01$; *** $p<0.001$. Statistical significance between untreated and treated MDR glioma cells (U87TxR) from three independent experiments $(n=3)$ : ${ }^{\# \#} p<0.01$; \#\#\# $p<0.001$

obtained by adaptation to chemotherapeutics. The selectivity of protoflavones toward the MDR cancer cell lines appeared to be independent from the origin of the cells as well as from the chemotherapeutic they were adapted to during the development of resistance. Importantly, compound 2 was selective toward cancer cells being significantly less effective in two human normal cell lines. Sensitivity of normal keratinocytes (HaCaT) to compound 5 was lower, while sensitivity of embryonal bronchial epithelial cells (MRC-5) to $\mathbf{6}$ was decreased. Their ability to distinguish normal cells differs, and new studies should be conducted to develop derivatives less toxic to normal cells. 
Even though studying the structure-activity relationships of protoflavone derivatives was not the scope of this work, it was observed that the 6-bromo-substituted compound 6 was the only one among the tested derivatives to which all of our human MDR cancer cell lines showed a significant CS. In contrast to this, compound $\mathbf{5}$, differing from $\mathbf{6}$ only in its 6-methyl substitution, was not selectively cytotoxic toward the DLD1-TxR cell line, while a similar selectivity toward NCI-H460/R and U87-TxR cells was observed as in case of compound $\mathbf{6}$. Based on their single-substituent structural difference and on the overview of their selectivity pattern on the employed cell lines, these two compounds were chosen for further studies of their mechanism of action using two pairs of non-MDR and MDR cells (NCIH460 and NCI-H460/R, and U87 and U87-TxR).

In contrast with the above-outlined cases of the human cancer cell line pairs, however, it was also observed that rat MDR glioma cells (RC6) were more resistant to protoflavones in comparison with their non-MDR counterparts (C6), which resistance was particularly strong in case of compound 4. This MDR cell line strengthened its intracellular antioxidative defense systems during the development of resistance [14].

Therefore, our data suggest that protoflavones might affect some of the mechanisms that were altered during the stepwise acquisition of P-gp-dependent resistance rather than the presence of the transporter itself. Considering that protoflavones have been described as pro-oxidants [8], our starting point was to examine whether the tested MDR cancer cells show different sensitivity to $\mathrm{H}_{2} \mathrm{O}_{2}$-induced oxidative stress when compared to their susceptible counterparts. Observations of sensitivity or resistance in case of the different cell lines to this treatment correlated well with those made for the protoflavones: The three human MDR cancer cell lines showed CS, while the RC6 cells were resistant to $\mathrm{H}_{2} \mathrm{O}_{2}$.

Next, we analyzed the mRNA expression of enzymes involved in the intracellular detoxification in non-MDR and MDR human NSCLC and glioma cells. Both MDR cancer cell lines possess lower mRNA GST $\pi$ and HIF- $1 \alpha$ expression than their corresponding non-MDR cell lines. It was previously suggested that decreased level of GST $\pi$ could be associated with MDR cells' vulnerability to pro-oxidants [22]. The MnSOD mRNA expression was decreased in NCI-H460/R cells, and it was elevated in U87-TxR cells. This suggests that NSCLC and glioma MDR cells developed different patterns of expression of antioxidative enzymes. Indeed, MnSOD expression could stabilize HIF-1 $\alpha$ protein levels [23], which in return may decrease its mRNA expression. We observed low MnSOD expression followed by low HIF- $1 \alpha$ expression in NCI-H460/R cells. According to Wang et al. [24], moderate overexpression of MnSOD, such as that observed in U87-TxR cells (less than fivefold compared to parental U87 cells), may block HIF- $1 \alpha$ protein accumulation. We assume that higher vulnerability to protoflavones is governed by lower GST $\pi$ activity in U87-TxR cells, while in NCI-H460/R cells, this is due to the insufficient activity of MnSOD. Although both MDR cancer cell lines possess P-gp overexpression, the alterations developed during adaptation to doxorubicin (NCI-H460/R) and paclitaxel (U87-TxR) differ [25]. Therefore, it is likely that the mechanisms behind lower antioxidative capacity in these two MDR cancer cell lines are distinct.

Importantly, we found that compounds $\mathbf{5}$ and $\mathbf{6}$ induced a decrease in G1 phase followed by arrest in G2/M phase of cell cycle in both NSCLC and glioma MDR cells. The same concentration of compounds did not affect the cell cycle in corresponding non-MDR cells. The effects of compounds 5 and $\mathbf{6}$ in MDR cells are in accordance with those observed previously in prostate, lung and colorectal carcinoma cell lines with protoapigenone (1) and its derivative WYC02-9 (2), which were shown to cause cell cycle perturbation and induce G2/M arrest [26-28]. Additionally, compound 6 significantly increased ROS/RNS production in glioma MDR cells and this was accompanied by a decrease in mRNA MnSOD and HIF- $1 \alpha$ expression. Although the level of GSH was increased in glioma MDR cells after protoflavones treatment, the expression of GST $\pi$ was considerably low which might have been insufficient to activate the GSH detoxification system. This is also in line with previous studies that revealed increased levels of ROS in prostate and breast cancer cells after treatment with $\mathbf{1}$ or $\mathbf{2}[9,26]$. Moreover, RY10-4, another synthetic protoflavone analog, was reported to down-regulate HIF- $1 \alpha$ expression in breast cancer cell lines [29].

Other authors showed that MnSOD activity is the highest during G1 phase and changes in its activity can significantly influence cellular redox status during the cell cycle [30]. Cellular redox state shifts toward a more oxidizing environment during $\mathrm{S}$ and $\mathrm{G} 2 / \mathrm{M}$ phases. Following cell division, the cellular redox state resets to that observed in the G1 phase. Inhibition of this oxidation state prior to $\mathrm{S}$ phase may negatively influence DNA synthesis [31]. Therefore, we assume that by lowering the portion of MDR cells in G1 phase and decreasing the MnSOD expression, compound $\mathbf{6}$ may induce accumulation of ROS during cell division and consequentially decrease the proliferation of MDR cells.

CS has been connected with hypersensitivity to oxidative stress in ABCG2 (BCRP) overexpressing MDR cancer cells: Krzyzanowski et al. [22], for example, found that MDCKII-BCRP cells are more vulnerable to ROS. As a possible explanation for the lower antioxidant capacity of these cells, lower glutathione (GSH) levels and decreased activities of glutathione-S-transferase (GST) 
and glutathione reductase (GR) were described. Lorendeau et al. [32] have recently published a comprehensive study on "classical" (i.e., not proto-) flavonoids' ability to selectively kill ABCC1 (MRP1)-transfected cancer cells through GSH depletion; potential use of protoflavones against such MDR cells might also be hypothesized. In addition to the role of oxidative stress in CS connected to the above-mentioned efflux transporters, Hall et al. [1] demonstrated that, in P-gp overexpressing MDR cells obtained via adaptation, CS activity of the thiol-containing prescription drug tiopronin was mediated by ROS generation and the inhibition of glutathione peroxidase (GPx).

However, these results obtained in vitro may not reflect the clinical situation. Therefore, in vivo preclinical models of MDR cancers should be engaged to confirm the CS to protoflavones. Likewise, several in vivo experiments confirmed the antitumor activity of compounds $\mathbf{1}$ and/or $\mathbf{2}$. In nude mice, $\mathbf{1}$ inhibited the growth of ovarian (MDAH-2774) and prostate (LNCap) cancer xenografts [33, 34], while both compounds exerted such activity against cervical (HeLa) cancer xenografts [35, 36]. Compound 2 was active against colorectal (HCT116) cancer xenografts in nude mice [28], and it was able to chemosensitize MDA-MB-231 breast cancer xenografts toward cisplatin possibly via the inhibition of DNA repair [10]. Moreover, 2 inhibited microvascularization in HeLa xenografts as well as in vivo angiogenesis both in zebrafish model and in nude mice [36].

In conclusion, we may argue that CS of MDR cancer cells to protoflavones is a non-P-gp-dependent ROS hypersensitivity [37]. Although our results together with those we obtained previously on a transfected cell line [11] suggest that the P-gp efflux pump itself plays little if any role in this phenomenon, development of MDR through P-gp overexpression led to the suppression of intracellular antioxidative capacity. This is apparently not significant in MDR cells that overexpress this efflux pump due to transfection with a vector containing P-gp, while the adaptation to a new stress situation (i.e., chemotherapeutic pressure) caused several further changes in addition to the P-gp overexpression in the MDR cancer cell lines utilized in our study. Therefore, we suggest that CS to protoflavone derivatives is mediated by hypersensitivity to ROS, which is not directly caused by P-gp overexpression, but other changes during the evolution of P-gp-mediated resistance. These results need to be confirmed in vivo on cancer models of MDR.

Acknowledgments This research was supported by the Ministry of Education, Science and Technological Development of the Republic of Serbia (Grant No III41031). The authors acknowledge the support from the National Science Council (NSC), Taiwan, the Szeged Foundation for Cancer Research and the Fundação para a Ciência e a Tecnologia (FCT), Portugal (PEsT-OE/SAU/UI0074/2011 and PEsT-OE/ SAU/UI0074/2014). This work was performed within the framework of COST Actions CM1106 (Chemical Approaches to Targeting Drug Resistance in Cancer Stem Cells; providing an STSM grant to A. Martins) and CM1407 (Challenging organic syntheses inspired by nature-from natural products chemistry to drug discovery). A bilateral mobility grant provided by the Hungarian Academy of Sciences and the NSC, Taiwan (MOST 104-2911-I-037-501), is also acknowledged.

\section{Compliance with ethical standards}

Conflict of interest None.

\section{References}

1. Hall MD, Marshall TS, Kwit AD, Miller Jenkins LM, Dulcey AE, Madigan JP, Pluchino KM, Goldsborough AS, Brimacombe KR, Griffiths GL, Gottesman MM (2014) Inhibition of glutathione peroxidase mediates the collateral sensitivity of multidrug-resistant cells to tiopronin. J Biol Chem 289(31):2147321489. doi:10.1074/jbc.M114.581702

2. Sharom FJ (2008) ABC multidrug transporters: structure, function and role in chemoresistance. Pharmacogenomics 9(1):105127. doi:10.2217/14622416.9.1.105

3. Gottesman MM (2002) Mechanisms of cancer drug resistance. Annu Rev Med 53:615-627. doi:10.1146/annurev. med.53.082901.103929

4. Hall MD, Handley MD, Gottesman MM (2009) Is resistance useless? Multidrug resistance and collateral sensitivity. Trends Pharmacol Sci 30(10):546-556. doi:10.1016/j.tips.2009.07.003

5. Pluchino KM, Hall MD, Goldsborough AS, Callaghan R, Gottesman MM (2012) Collateral sensitivity as a strategy against cancer multidrug resistance. Drug Resist Updat 15(1-2):98-105. doi:10.1016/j.drup.2012.03.002

6. Szakacs G, Hall MD, Gottesman MM, Boumendjel A, Kachadourian R, Day BJ, Baubichon-Cortay H, Di Pietro A (2014) Targeting the Achilles heel of multidrug-resistant cancer by exploiting the fitness cost of resistance. Chem Rev 114(11):5753-5774. doi:10.1021/cr4006236

7. Nakagawa-Goto K, Chang PC, Lai CY, Hung HY, Chen TH, Wu PC, Zhu H, Sedykh A, Bastow KF, Lee KH (2010) Antitumor agents. 280. Multidrug resistance-selective desmosdumotin B analogues. J Med Chem 53(18):6699-6705. doi:10.1021/jm100846r

8. Hunyadi A, Martins A, Danko B, Chang FR, Wu YC (2014) Protoflavones: a class of unusual flavonoids as promising novel anticancer agents. Phytochem Rev 13(1):69-77. doi:10.1007/ s11101-013-9288-2

9. Chen WY, Hsieh YA, Tsai CI, Kang YF, Chang FR, Wu YC, Wu CC (2011) Protoapigenone, a natural derivative of apigenin, induces mitogen-activated protein kinase-dependent apoptosis in human breast cancer cells associated with induction of oxidative stress and inhibition of glutathione S-transferase pi. Invest New Drugs 29(6):1347-1359. doi:10.1007/s10637-010-9497-0

10. Wang HC, Lee AY, Chou WC, Wu CC, Tseng CN, Liu KY, Lin WL, Chang FR, Chuang DW, Hunyadi A, Wu YC (2012) Inhibition of ATR-dependent signaling by protoapigenone and its derivative sensitizes cancer cells to interstrand cross-link-generating agents in vitro and in vivo. Mol Cancer Ther 11(7):14431453. doi:10.1158/1535-7163.MCT-11-0921

11. Danko B, Martins A, Chuang DW, Wang HC, Amaral L, Molnar J, Chang FR, Wu YC, Hunyadi A (2012) In vitro cytotoxic activity of novel protoflavone analogs - selectivity towards a multidrug resistant cancer cell line. Anticancer Res 32(7):2863-2869

12. Pesic M, Markovic JZ, Jankovic D, Kanazir S, Markovic ID, Rakic L, Ruzdijic S (2006) Induced resistance in the human 
non small cell lung carcinoma (NCI-H460) cell line in vitro by anticancer drugs. J Chemother 18(1):66-73. doi:10.1179/ joc.2006.18.1.66

13. Podolski-Renic A, Andelkovic T, Bankovic J, Tanic N, Ruzdijic S, Pesic M (2011) The role of paclitaxel in the development and treatment of multidrug resistant cancer cell lines. Biomed Pharmacother 65(5):345-353. doi:10.1016/j.biopha.2011.04.015

14. Stojkovic S, Podolski-Renic A, Dinic J, Stankovic T, Bankovic J, Hadzic S, Paunovic V, Isakovic A, Tanic N, Pesic M (2015) Development of resistance to antiglioma agents in rat C6 cells caused collateral sensitivity to doxorubicin. Exp Cell Res. doi:10.1016/j.yexcr.2015.05.018

15. Hunyadi A, Chuang DW, Danko B, Chiang MY, Lee CL, Wang HC, Wu CC, Chang FR, Wu YC (2011) Direct semi-synthesis of the anticancer lead-drug protoapigenone from apigenin, and synthesis of further new cytotoxic protoflavone derivatives. PLoS ONE 6(8):e23922. doi:10.1371/journal.pone.0023922

16. O'Driscoll L, Daly C, Saleh M, Clynes M (1993) The use of reverse transcriptase-polymerase chain reaction (RT-PCR) to investigate specific gene expression in multidrug-resistant cells. Cytotechnology 12(1-3):289-314

17. Larrea E, Beloqui O, Munoz-Navas MA, Civeira MP, Prieto J (1998) Superoxide dismutase in patients with chronic hepatitis C virus infection. Free Radic Biol Med 24(7-8):1235-1241

18. Nardinocchi L, Puca R, Sacchi A, D’Orazi G (2009) Inhibition of HIF-1alpha activity by homeodomain-interacting protein kinase-2 correlates with sensitization of chemoresistant cells to undergo apoptosis. Mol Cancer 8:1. doi:10.1186/1476-4598-8-1

19. Livak KJ, Schmittgen TD (2001) Analysis of relative gene expression data using real-time quantitative PCR and the 2(-Delta Delta C(T)) method. Methods 25(4):402-408. doi:10.1006/meth.2001.1262

20. NicAmhlaoibh R, Heenan M, Cleary I, Touhey S, O'Loughlin C, Daly C, Nunez G, Scanlon KJ, Clynes M (1999) Altered expression of mRNAs for apoptosis-modulating proteins in a low level multidrug resistant variant of a human lung carcinoma cell line that also expresses mdr1 mRNA. Int J Cancer 82(3):368-376

21. Prochazkova J, Kubala L, Kotasova H, Gudernova I, Sramkova Z, Pekarova M, Sarkadi B, Pachernik J (2011) ABC transporters affect the detection of intracellular oxidants by fluorescent probes. Free Radic Res 45(7):779-787. doi:10.3109/10715762.2 011.579120

22. Krzyzanowski D, Bartosz G, Grzelak A (2014) Collateral sensitivity: ABCG2-overexpressing cells are more vulnerable to oxidative stress. Free Radic Biol Med 76:47-52. doi:10.1016/j. freeradbiomed.2014.07.020

23. Kaewpila S, Venkataraman S, Buettner GR, Oberley LW (2008) Manganese superoxide dismutase modulates hypoxia-inducible factor-1 alpha induction via superoxide. Cancer Res 68(8):27812788. doi:10.1158/0008-5472.CAN-07-2635

24. Wang M, Kirk JS, Venkataraman S, Domann FE, Zhang HJ, Schafer FQ, Flanagan SW, Weydert CJ, Spitz DR, Buettner GR, Oberley LW (2005) Manganese superoxide dismutase suppresses hypoxic induction of hypoxia-inducible factor-1alpha and vascular endothelial growth factor. Oncogene 24(55):8154-8166. doi:10.1038/sj.onc.1208986

25. Podolski-Renic A, Jadranin M, Stankovic T, Bankovic J, Stojkovic S, Chiourea M, Aljancic I, Vajs V, Tesevic V, Ruzdijic S, Gagos S, Tanic N, Pesic M (2013) Molecular and cytogenetic changes in multi-drug resistant cancer cells and their influence on new compounds testing. Cancer Chemother Pharmacol 72(3):683-697. doi:10.1007/s00280-013-2247-1

26. Chen HM, Chang FR, Hsieh YC, Cheng YJ, Hsieh KC, Tsai LM, Lin AS, Wu YC, Yuan SS (2011) A novel synthetic protoapigenone analogue, WYC02-9, induces DNA damage and apoptosis in DU145 prostate cancer cells through generation of reactive oxygen species. Free Radic Biol Med 50(9):1151-1162. doi:10.1016/j.freeradbiomed.2011.01.015

27. Chiu CC, Chang HW, Chuang DW, Chang FR, Chang YC, Cheng YS, Tsai MT, Chen WY, Lee SS, Wang CK, Chen JY, Wang HM, Chen CC, Liu YC, Wu YC (2009) Fern plant-derived protoapigenone leads to DNA damage, apoptosis, and $\mathrm{G}(2) / \mathrm{m}$ arrest in lung cancer cell line H1299. DNA Cell Biol 28(10):501-506. doi:10.1089/dna.2009.0852

28. Chen YJ, Chen HP, Cheng YJ, Lin YH, Liu KW, Chen YJ, Hou MF, Wu YC, Lee YC, Yuan SS (2013) The synthetic flavonoid WYC02-9 inhibits colorectal cancer cell growth through ROSmediated activation of MAPK14 pathway. Life Sci 92(22):10811092. doi:10.1016/j.lfs.2013.04.007

29. Liu Z, Yuan Q, Zhang X, Xiong C, Xue P, Ruan J (2012) RY104, a novel anti-tumor compound, exhibited its anti-angiogenesis activity by down-regulation of the HIF-1alpha and inhibition phosphorylation of AKT and mTOR. Cancer Chemother Pharmacol 69(6): 1633-1640. doi:10.1007/s00280-012-1873-3

30. Sarsour EH, Kalen AL, Goswami PC (2014) Manganese superoxide dismutase regulates a redox cycle within the cell cycle. Antioxid Redox Signal 20(10):1618-1627. doi:10.1089/ars.2013.5303

31. Menon SG, Sarsour EH, Spitz DR, Higashikubo R, Sturm M, Zhang H, Goswami PC (2003) Redox regulation of the G1 to S phase transition in the mouse embryo fibroblast cell cycle. Cancer Res 63(9):2109-2117

32. Lorendeau D, Dury L, Genoux-Bastide E, Lecerf-Schmidt F, Simoes-Pires C, Carrupt PA, Terreux R, Magnard S, Di Pietro A, Boumendjel A, Baubichon-Cortay H (2014) Collateral sensitivity of resistant MRP1-overexpressing cells to flavonoids and derivatives through GSH efflux. Biochem Pharmacol 90(3):235245. doi:10.1016/j.bcp.2014.05.017

33. Chang HL, Su JH, Yeh YT, Lee YC, Chen HM, Wu YC, Yuan SS (2008) Protoapigenone, a novel flavonoid, inhibits ovarian cancer cell growth in vitro and in vivo. Cancer Lett 267(1):85-95. doi:10.1016/j.canlet.2008.03.007

34. Chang HL, Wu YC, Su JH, Yeh YT, Yuan SS (2008) Protoapigenone, a novel flavonoid, induces apoptosis in human prostate cancer cells through activation of $\mathrm{p} 38$ mitogen-activated protein kinase and c-Jun NH2-terminal kinase 1/2. J Pharmacol Exp Ther 325(3):841-849. doi:10.1124/jpet.107.135442

35. Chen YJ, Kay N, Yang JM, Lin CT, Chang HL, Wu YC, Fu CF, Chang Y, Lo S, Hou MF, Lee YC, Hsieh YC, Yuan SS (2013) Total synthetic protoapigenone WYC02 inhibits cervical cancer cell proliferation and tumour growth through PIK3 signalling pathway. Basic Clin Pharmacol Toxicol 113(1):8-18. doi:10.1111/bcpt.12057

36. Chen YJ, Cheng YJ, Hung AC, Wu YC, Hou MF, Tyan YC, Yuan SS (2013) The synthetic flavonoid WYC02-9 inhibits cervical cancer cell migration/invasion and angiogenesis via MAPK14 signaling. Gynecol Oncol 131(3):734-743. doi:10.1016/j. ygyno.2013.10.012

37. Chen J (2014) Reactive oxygen species and drug resistance in cancer chemotherapy. Austin J Clin Pathol 1(4):1017 


\section{CHEMMEDCHEM}

CHEMISTRY ENABLING DRUG DISCOVERY
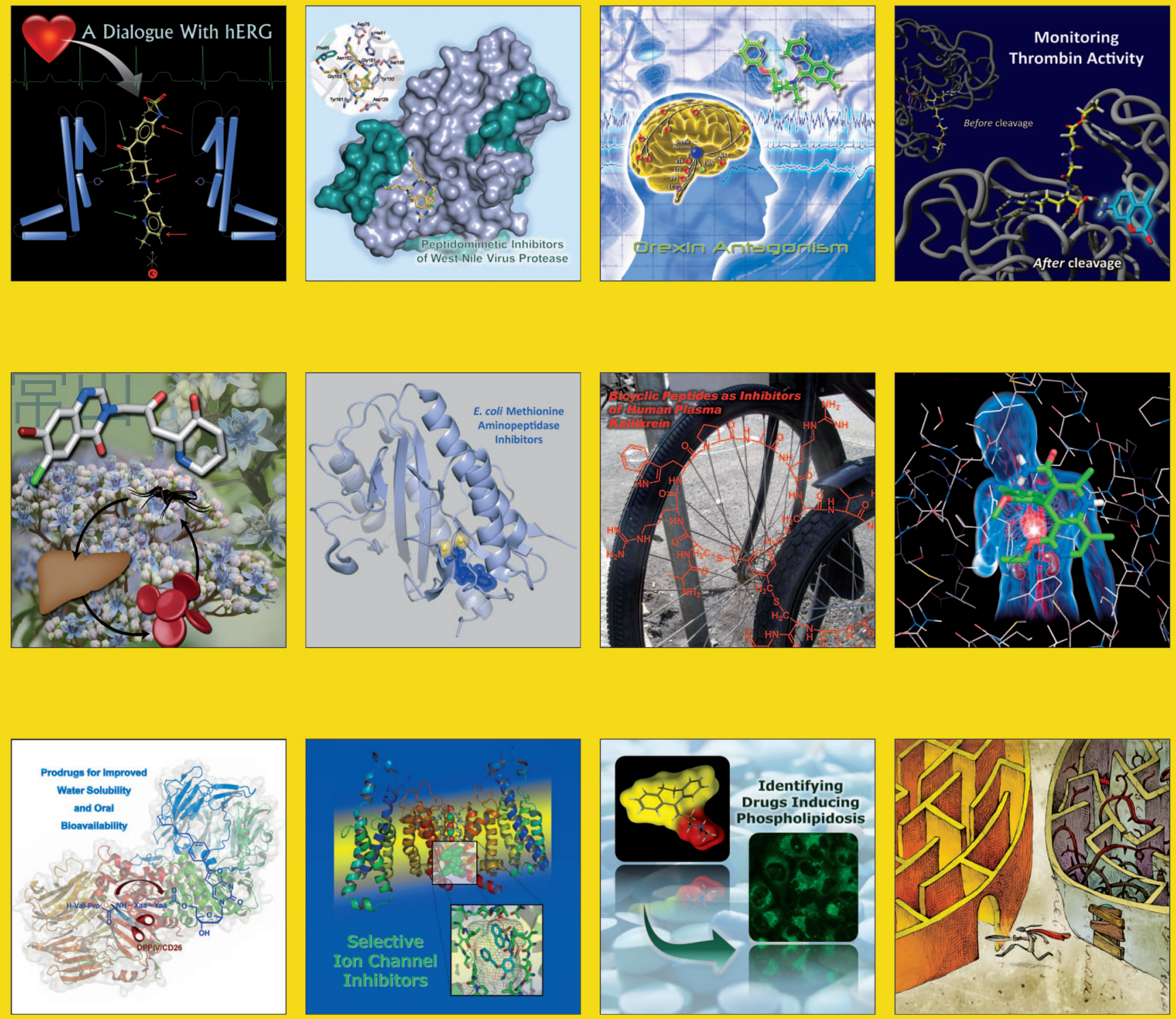

\section{Reprint}

(c) Wiley-VCH Verlag GmbH \& Co. KGaA, Weinheim

A Journal of 


\title{
Synthesis and SAR Study of Anticancer Protoflavone Derivatives: Investigation of Cytotoxicity and Interaction with $A B C B 1$ and ABCG2 Multidrug Efflux Transporters
}

\author{
Balázs Dankó ${ }^{+}{ }^{[a]}$ Szilárd Tóth ${ }^{+}{ }^{[b]}$ Ana Martins, ${ }^{[c, k]}$ Máté Vágvölgyi ${ }_{1}^{[a]}$ Norbert Kúsz, ${ }^{[a]}$ \\ Joseph Molnár, ${ }^{[c]}$ Fang-Rong Chang, ${ }^{[\mathrm{d}, \mathrm{e}, \mathrm{fl}}$ Yang-Chang Wu, ${ }^{[\mathrm{d}, \mathrm{g}, \mathrm{h]}}{ }^{\mathrm{G}}$ Gergely Szakács, ${ }^{[\mathrm{b}, \mathrm{i}}$ and \\ Attila Hunyadi*[a, j]
}

\begin{abstract}
There is a constant need for new therapies against multidrugresistant (MDR) cancer. Natural compounds are a promising source of novel anticancer agents. We recently showed that protoflavones display activity in MDR cancer cell lines that overexpress the P-glycoprotein (P-gp) drug efflux pump. In this study, 52 protoflavones, including 22 new derivatives, were synthesized and tested against a panel of drug-sensitive parental cells and their MDR derivatives obtained by transfection with the human $A B C B 1$ or $A B C G 2$ genes, or by adaptation to
\end{abstract}

chemotherapeutics. With the exception of protoapigenone, identified as a weak ABCG2 substrate, all protoflavones bypass resistance conferred by these two transporters. The majority of the compounds were found to exhibit mild to strong (up to 13-fold) selectivity against the MCF-7 ${ }_{\text {Dox }}$ and KB-V1 cell lines, but not to transfected MDR cells engineered to overexpress the MDR transporters. Our results suggest that protoflavones can overcome MDR cancer by evading P-gp-mediated efflux.

\section{Introduction}

Cancer is among the leading causes of morbidity and mortality worldwide. According to the World Cancer Report, 8.2 million cancer-related deaths were registered in 2012, and it is expected that annual cancer cases will rise from 14 million in 2012 to around 22 million within the next two decades. ${ }^{[1]}$ Resistance to chemotherapy and molecularly targeted therapies is a major problem facing current cancer research. ${ }^{[2]}$ Despite the availability of a broad range of diverse anticancer compounds with new mechanisms and molecular targets, cancer is often incurable due to the development of drug resistance. ${ }^{[3]}$ Resistance can rapidly develop even in cases when the tumor initially responds to chemotherapy. Multi-drug resistance (MDR) can emerge as a result of decreased uptake or increased efflux of cytostatic agents-the latter is mediated by ATP-binding-cassette $(A B C)$ proteins, primarily by P-glycoprotein (P-gp; $A B C B 1)$ and $A B C G 2$, which confer resistance to a wide variety of compounds. ${ }^{[4,5]}$ There is a constant need for novel chemotherapeutics with marked and selective antitumor activity that can overcome resistance mediated by these transporters. The unfavorable prognostic impact of P-gp expression in several cancers has prompted overwhelming research efforts aimed at the clinical development of high affinity efflux inhibitors that were shown to overcome MDR in in vitro models. Unfortunately, even after decades of intensive research, a clinically effective inhibitor has not been identified. Recently, the discussion has shifted to alternative strategies, either to bypass the transport- [a] B. Dankó, ${ }^{+}$M. Vágvölgyi, N. Kúsz, Dr. A. Hunyadi

Institute of Pharmacognosy, University of Szeged, Szeged (Hungary)

E-mail:hunyadi.a@pharm.u-szeged.hu

[b] S. Tóth, ${ }^{+}$Dr. G. Szakács

Institute of Enzymology, Research Centre for Natural Sciences, Hungarian Academy of Sciences, Budapest (Hungary)

[c] Dr. A. Martins, Prof. J. Molnár

Department of Medical Microbiology and Immunobiology, Faculty of Medicine, University of Szeged, Szeged (Hungary)

[d] Prof. F.-R. Chang, Prof. Y.-C. Wu

Graduate Institute of Natural Products, Kaohsiung Medical University, Kaohsiung, (Taiwan, R.O.C.)

[e] Prof. F.-R. Chang

Cancer Center, Kaohsiung Medical University Hospital, Kaohsiung, (Taiwan, R.O.C.)

[f] Prof. F.-R. Chang

$R \& D$ Center of Chinese Herbal Medicines \& New Drugs, College of Pharmacy, Kaohsiung Medical University, Kaohsiung, (Taiwan, R.O.C.) [g] Prof. Y.-C. Wu

Research Center for Natural Products and Drug Development, Kaohsiung Medical University, Kaohsiung (Taiwan, R.O.C.)

[h] Prof. Y.-C. Wu

Department of Medical Research, Kaohsiung Medical University Hospital, Kaohsiung, (Taiwan, R.O.C.)

[i] Dr. G. Szakács

Institute of Cancer Research, Medical University Vienna, Vienna (Austria)

[j] Dr. A. Hunyadi

Interdisciplinary Centre for Natural Products, University of Szeged, Szeged (Hungary)

[k] Dr. A. Martins

Current address: Synthetic Systems Biology Unit, Institute of Biochemistry, Biological Research Centre, Temesvári krt. 62, 6726 Szeged (Hungary)

$\left.{ }^{+}\right]$These authors contributed equally to this work.

$\square$ Supporting information and the ORCID identification number(s) for the

(D) author(s) of this article can be found under https://doi.org/10.1002/ cmdc. 201700225 . 
ers or to exploit the collateral sensitivity (CS) of MDR cells. ${ }^{[6]}$ Recent discoveries have shown that it is possible to invert the selective advantage of resistant cells to reverse the evolution of resistance. ${ }^{[7]}$ For example, MDR-selective compounds were shown to specifically target $A B C$ transporter overexpressing MDR cancer cells by exploiting the Achilles' heel conferred by the overexpression of the transporters. ${ }^{[8-10]}$

Our review of the literature identified several natural compounds that were reported to elicit preferential toxicity against MDR cells. ${ }^{[6]}$ For example, the $4^{\prime}$-hydroxyflavone apigenin was identified in a screen as a specific killer of drug-selected H69AR cells and MRP1-transfected HeLa cells. ${ }^{[11]}$ Flavonoids are naturally derived compounds that display both anti- and pro-oxidant properties. Flavonoids have been used in cancer chemoprevention and chemotherapy. A particularly interesting, rare group of natural flavonoids with a high antitumor potential is that of protoflavones. Typically derived from ferns, protoflavones contain a nonaromatic, usually p-quinol B-ring or its dior tetrahydro derivative. Based on the most frequently occurring chemical nomenclature, herein we refer to the flavone skeleton containing a $1^{\prime}$-hydroxy group and a $2^{\prime}, 5^{\prime}$-dien-4'-one moiety in its B-ring as the "protoflavone" skeleton. Protoflavones can formally be derived from 4'-hydroxyflavones, like apigenin (1), and some, for example, protoapigenone (2), the protoflavone analogue of 1, have been described as potent anticancer agents in vitro and in vivo. We have recently reviewed the chemistry and bioactivity of protoflavones. ${ }^{[12]}$ The proapoptotic activity of protoflavones is mediated by oxidative stress $^{[13]}$ and the inhibition of ATR-dependent signaling. ${ }^{[14]}$ We have previously shown that 6-methylated protoflavone derivatives exert mild selective cytotoxicity against a murine lymphoma cell line transfected with the human $A B C B 1$ transporter, while other protoflavones, derived from apigenin, genkwanin or $\beta$-naphthoflavone, did not exhibit such selectivity. ${ }^{[15]}$ Furthermore, protoapigenone and its 1 '-O-butyl- and propargylether, the $\beta$-naphthoflavone analogue WYC0209, 6-methylprotoflavone and 6-bromoprotoflavone showed selective cytotoxicity against certain MDR cancer cell lines, such as $\mathrm{NCl}-\mathrm{H} 460$ human non-small cell lung carcinoma cells adapted to doxorubicin, U87 human glioma and DLD1 human colorectal cells, both adapted to paclitaxel. ${ }^{[16]}$ On the other hand, cross-resistance (CR) to protoflavones was observed in $\mathrm{C} 6$ rat glioma cells adapted to carmustine, and CS/CR pattern appeared to be in line with altered antioxidative capacity of the MDR cells relative to their parental cell lines. ${ }^{[16]}$

Based on these results our aim was to systematically explore the cytotoxicity and antitumor potential of further protoflavone derivatives. In particular, we characterized the anticancer activity of a total of 52 compounds in a diverse panel of cancer cell lines including MDR derivatives expressing $A B C B 1$ or $A B C G 2$.

\section{Results}

Thirty-seven protoflavones and protoflavone $1^{\prime}$-O-alkyl ethers were synthesized from apigenin (1), genkwanin, 4'-hydroxy-6methylflavone, 4'-hydroxy-6-methoxyflavone and 4'-hydroxy- $\beta$ naphthoflavone, based on the synthetic route we have previ- ously published for compounds $2-\mathbf{9}_{,}^{[17]} 11-24^{[15]}$ and 31-38. ${ }^{[17]}$ Briefly, an oxidative de-aromatization was performed by a common hypervalent iodine reagent, [bis(trifluoroacetoxy)iodo]benzene (PIFA) in acetonitrile in the presence of water or the alcohol to be coupled at position $\mathrm{C}^{\prime}$. Among these compounds, protoapigenone 1'-O-benzylether 10 and the 6-methoxylated derivatives 25-30 were obtained as new protoflavones; the synthesis and structures of the compounds are presented in Scheme 1.

Total synthesis of a set of various 6-substituted protoflavones was achieved in four to six steps. To obtain starting materials (i.e., 5'-ethyl-2'-hydroxyacetophenone, 41; and 5'-pentyl2'-hydroxyacetophenone; 42) for our 6-ethyl- and 6-pentylsubstituted target compounds, the appropriate $p$-substituted phenols were acetylated and subjected to Fries rearrangement reaction under the condition of dry aluminum chloride in dichloromethane. ${ }^{[18]}$ The resulting 2 '-hydroxyacetophenones and those commercially available with a 5'-ethoxy or 5'-bromo substituent (43 and $\mathbf{4 4}$, respectively) were used in Claisen-Schmidt condensation reactions with $p$-benzyloxybenzaldehyde to yield chalcones 45-48, which, after performing ring closure with iodine in DMSO, yielded the corresponding 6-substituted 4'benzyloxyflavones 49-52. The 6-bromo-substituted compound 52 was subjected to Suzuki coupling to obtain the corresponding 6-phenylflavone 53. Debenzylation of the flavonoids obtained this way and subsequent oxidative de-aromatization of the flavones 54-58 with PIFA, as described above, allowed us to obtain the protoflavones with various substituents at positions C6 and C1' (59-73). Scheme 2 summarizes the total synthetic procedure.

In the first set of experiments, the cytotoxicity of compounds 2-38 and 59-73 were tested in two drug-sensitive/ MDR cancer cell line pairs (parental L5178 mouse lymphoma cells and $\mathrm{L} 517 \mathrm{~B}_{\mathrm{B} 1}$ cells engineered to overexpress the human $A B C B 1$ protein; parental MCF-7 breast cancer cells and the doxorubicin resistant derivative MCF-7 $_{\text {Dox }}$ overexpressing Pgp). The fraction of $\mathrm{IC}_{50}$ values obtained in P-gp negative vs. positive cells served as a quantification of the MDR selective effect (selectivity ratio, SR). Accordingly, $S R \leq 0.5$ indicated that the compound is subject to P-gp-mediated resistance, whereas $\mathrm{SR} \geq 2$ suggested that the P-gp expressing cells demonstrate collateral sensitivity against the tested protoflavone derivative. Results of the cytotoxicity testing on the L5178 and MCF-7 models are summarized in Figure 1; detailed data are presented in Supporting Information Table S1.

The above results indicate that the synthesized protoflavone derivatives possess significant toxicity in the two cell line pairs. Interestingly, MCF7 cells were in general more resistant. Whereas the expression of $A B C B 1$ did not modify the sensitivity of L5178 ${ }_{B}$ cells in comparison with the parental L5178 cell line, MCF-7 Dox cells showed collateral sensitivity to several derivatives, with SR values exceeding 5 in the case of compounds $16,18-22,37,68-71$, and 73.

To substantiate the role of $A B C$ transporters in the MDR-selective toxicity of the compounds, additional MDR models were included in the study. The cytotoxic activity of compounds 2-11, 18, 31-38, 66 and 68-73 were tested in four ad- 
<smiles>[R]c1cc2oc(-c3ccc(O)cc3)cc(=O)c2c([R])c1[R]</smiles>

Apigenin (1) $\mathrm{R}^{1}=\mathrm{OH}, \mathrm{R}^{2}=\mathrm{H}, \mathrm{R}^{3}=\mathrm{OH}$ Genkwanin $\mathrm{R}^{1}=\mathrm{OMe}, \mathrm{R}^{2}=\mathrm{H}, \mathrm{R}^{3}=\mathrm{OH}$

4'-Hydroxy-6-methylflavone $\mathrm{R}^{1}=\mathrm{H}, \mathrm{R}^{2}=\mathrm{Me}, \mathrm{R}^{3}=\mathrm{H}$

4'-Hydroxy-6-methoxyflavone $\mathrm{R}^{1}=\mathrm{H}, \mathrm{R}^{2}=\mathrm{OMe}, \mathrm{R}^{3}=\mathrm{H}$

\section{a}<smiles>[R]c1cc2oc(C3([R])C=CC(=O)C=C3)cc(=O)c2c([R])c1[R]</smiles>

$\mathrm{R}^{1}=\mathrm{OH}, \mathrm{R}^{2}=\mathrm{H}, \mathrm{R}^{3}=\mathrm{OH}$

2: $\mathrm{R}=\mathrm{H} ; 31.2 \% \quad$ 7: $\mathrm{R}=\mathrm{Bu} ; 44.0 \%$

3: $\mathrm{R}=\mathrm{Me} ; 42.0 \% \quad$ 8: $\mathrm{R}=$ allyl; $29.1 \%$

4: R=Et; $33.1 \% \quad$ 9: $R=$ propargyl; $35.9 \%$

5: $\mathrm{R}=\mathrm{Pr} ; 36.0 \% \quad 10: \mathrm{R}=\mathrm{Bn} ; 39.6 \%$

6: $R=i P r ; 25.0 \%$

$\mathrm{R}^{1}=\mathrm{OMe}, \mathrm{R}^{2}=\mathrm{H}, \mathrm{R}^{3}=\mathrm{OH}$

11: $\mathrm{R}=\mathrm{H} ; 57.0 \%$

12: $\mathrm{R}=\mathrm{Me} ; 62.0 \%$

13: $\mathrm{R}=\mathrm{Et} ; 59.2 \%$

14: $\mathrm{R}=\mathrm{Pr} ; 52.8$

15: $\mathrm{R}=\mathrm{Bu} ; 33.0 \%$

16: $R=$ allyl; $43.6 \%$

17: R=propargyl; $37.2 \%$

$\mathrm{R}^{1}=\mathrm{H}, \mathrm{R}^{2}=\mathrm{Me}, \mathrm{R}^{3}=\mathrm{H}$

18: $\mathrm{R}=\mathrm{H} ; 48.2 \%$

19: $R=M e ; 59.7 \%$

20: $R=E t ; 20.0 \%$

21: $R=P r ; 36.0 \%$

22: $\mathrm{R}=\mathrm{Bu} ; 35.0 \%$

23: $\mathrm{R}=$ allyl; $43.0 \%$

24: $\mathrm{R}=$ propargyl; $42.0 \%$
$\mathrm{R}^{1}=\mathrm{H}, \mathrm{R}^{2}=\mathrm{OMe}, \mathrm{R}^{3}=\mathrm{H}$

25: $\mathrm{R}=\mathrm{H} ; 32.3 \%$

26: $\mathrm{R}=\mathrm{Me} ; 41.3 \%$

27: $\mathrm{R}=\mathrm{Et} ; 39.3 \%$

28: $R=B u ; 46.3 \%$

29: $R=$ allyl; $37.2 \%$

30: R=propargyl; $37.7 \%$<smiles>CC(C)C</smiles>

4'-hydroxy- $\beta$-naphthoflavone<smiles>[R]C1(c2cc(=O)c3c(ccc4ccccc43)o2)C=CC(=O)C=C1</smiles>

31: $\mathrm{R}=\mathrm{H}$;

32: $\mathrm{R}=\mathrm{Me}$

33: $\mathrm{R}=\mathrm{Et} ; 65.0 \%$

34: $\mathrm{R}=\mathrm{Pr} ; 64.1 \%$

35: $\mathrm{R}=\mathrm{iPr} ; 51.6 \%$

36: $\mathrm{R}=\mathrm{Bu} ; 54.0 \%$

37: R=allyl; $50.5 \%$

38: $R=$ propargyl; $44.8 \%$

Scheme 1. Synthesis of protoflavones from commercially available 4'-hydroxyflavones: a) $\mathrm{CH}_{3} \mathrm{CN} / \mathrm{ROH}$ 9:1, PIFA (2 equiv).

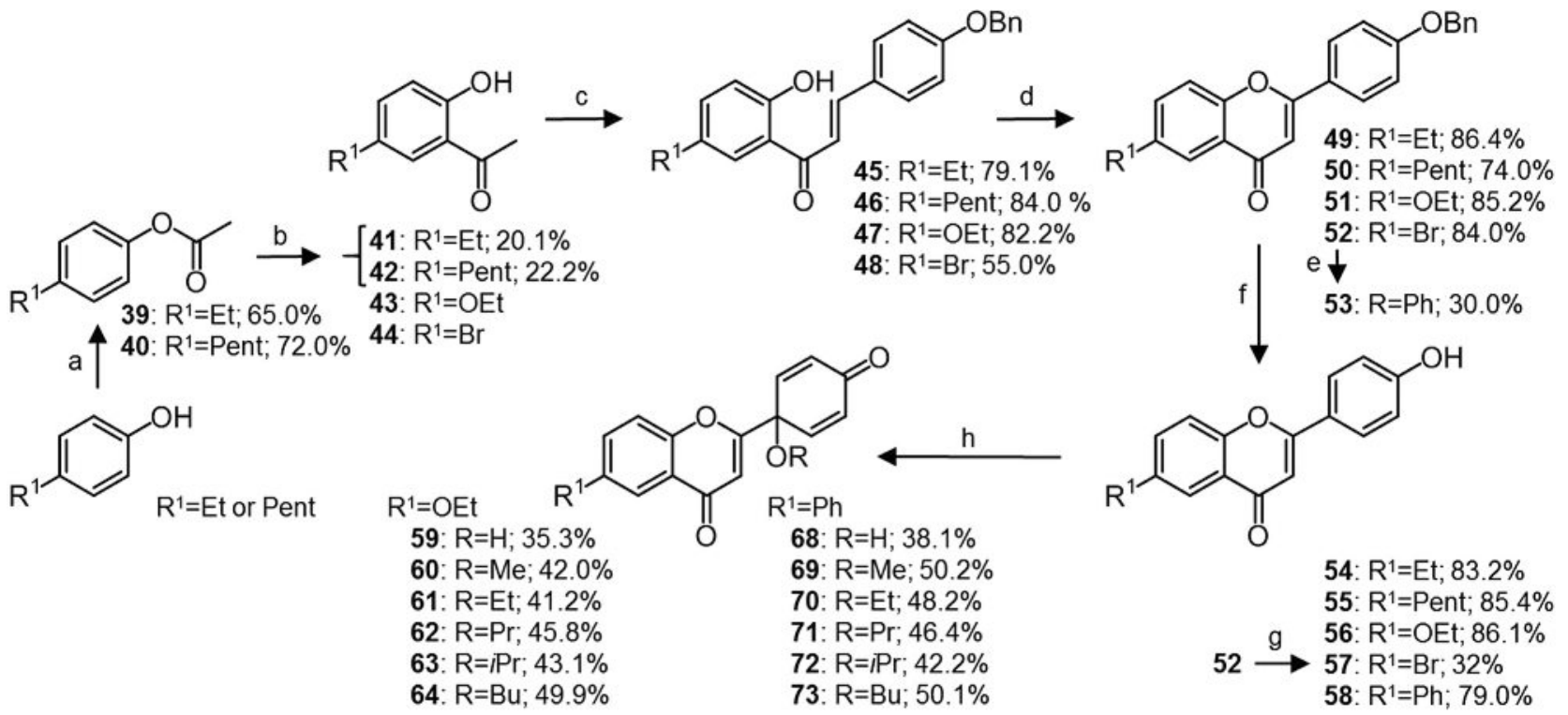

65: $\mathrm{R}^{1}=\mathrm{Et}, \mathrm{R}=\mathrm{H} ; \mathbf{4 1 . 0 \%}$

66: $\mathrm{R}^{1}=$ Pent, $\mathrm{R}=\mathrm{H} ; 42.3 \%$

67: $\mathrm{R}^{1}=\mathrm{Br}, \mathrm{R}=\mathrm{H} ; 40.0 \%$

Scheme 2. Total synthesis of 6-substituted protoflavone derivatives: a) $\left(\mathrm{CH}_{3} \mathrm{CO}\right)_{2} \mathrm{O}$, conc. $\left.\left.\mathrm{H}_{2} \mathrm{SO}_{4} ; \mathrm{b}\right) \mathrm{AlCl}_{3} ; \mathrm{C}\right) \mathrm{EtOH}, 4-\mathrm{benzyloxybenzaldehyde}, 50 \% \mathrm{KOH} / \mathrm{H}_{2} \mathrm{O}$; d) $\mathrm{I}_{2}$, DMSO; e) phenylboronic acid, $\mathrm{K}_{2} \mathrm{CO}_{3}$, tetrakis(triphenylphosphine)palladium(0); f) $\left.10 \% \mathrm{Pd} / \mathrm{C}_{2} \mathrm{H}_{2} ; \mathrm{g}\right) \mathrm{TFA} / \mathrm{CH}_{2} \mathrm{Cl}_{2}, \mathrm{reflux}^{[16]}$; h) $\mathrm{CH}_{3} \mathrm{CN} / \mathrm{ROH}$ :1, $\mathrm{PIFA}$ (2 equiv).

ditional drug-sensitive/MDR cell line pairs, including $A 431$, $\mathrm{A} 431_{\mathrm{B} 1}, \mathrm{~A} 431_{\mathrm{G} 2}, \mathrm{MES}-\mathrm{SA}, \mathrm{MES}-\mathrm{SA} / \mathrm{Dx} 5, \mathrm{~KB}-3-1$ and KB-V1. These compounds represent a diverse sub-set of derivatives of the naturally occurring protoapigenone (2) and protogenkwanone (11), analogues of the synthetic WYC0209 (31) identified as a potential lead in previous studies, ${ }^{[12]}$ as well as 6-methyl, 6pentyl, and 6-phenyl derivatives $(18,66$, and 68-73) aiming to further explore structure-activity relationships (SAR) at C6. The results are shown in Figures 2 and 3; detailed data are available as Supporting Information (Tables S2 and S3).

The tested protoflavone derivatives were equally toxic to $\mathrm{A} 431, \mathrm{~A} 431_{\mathrm{G} 2}$ or $\mathrm{A} 431_{\mathrm{B} 1}$ cells $\left(\mathrm{IC}_{50}\right.$ values ranged from 0.60 to $7.27 \mu \mathrm{M}$ ), with the exception of protoapigenone (2), suggesting that the compounds tested herein are able to bypass $A B C B 1$ 

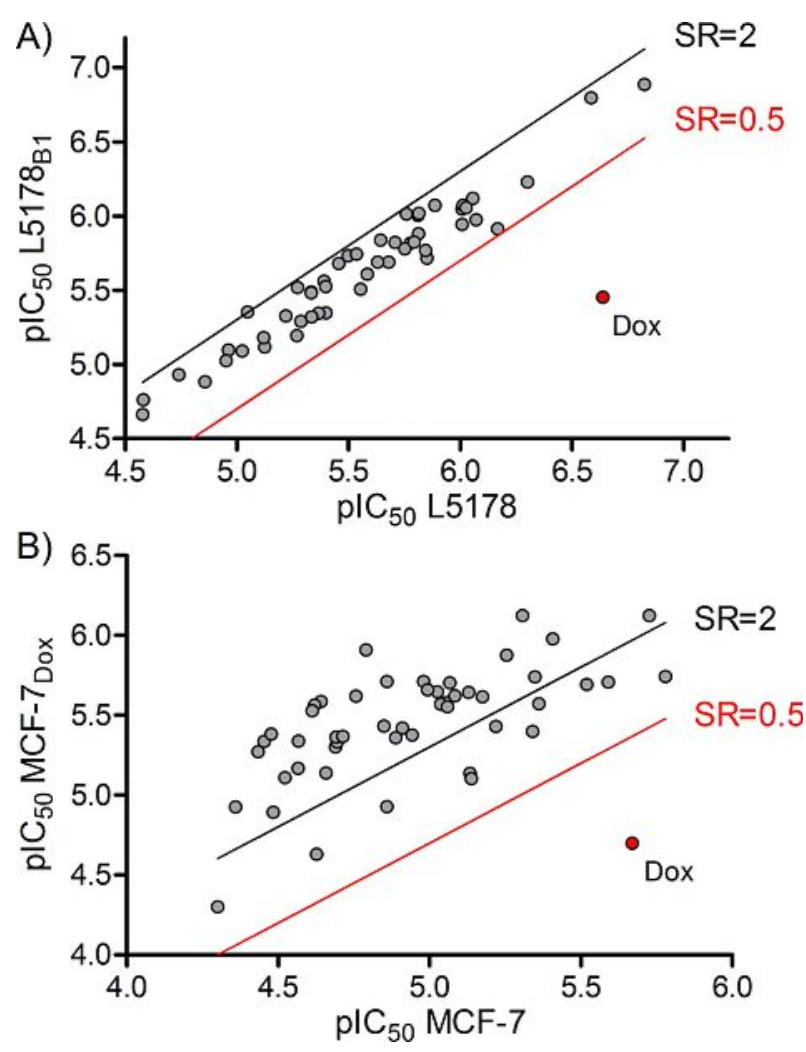

Figure 1. Cytotoxicity of protoflavones 2-38 and 59-73. $\mathrm{plC}_{50}$ values were derived from dose-response curves obtained from cell viability experiments on A) L5178/L5178 ${ }_{B 1}$ mouse lymphoma cells and B) MCF-7/MCF-7 Dox cells. $\mathrm{SR}=$ selectivity ratio, calculated as $I C_{50}{ }^{\text {non-MDR }} / \mathrm{IC}_{50}{ }^{\mathrm{MDR}} ; n=3-4$; Dox: doxorubicin.

as well as $\mathrm{ABCG} 2$. Resistance of $\mathrm{A} 431_{\mathrm{G} 2}$ cells to compound 2 was abolished in the presence of tariquidar, confirming that protoapigenone is an ABCG2 substrate (Figure 2).

Finally, we tested the interaction of the compounds with Pgp to reveal if any of the compounds inhibit drug efflux. $A B C B 1$ function was characterized using the calcein accumulation assay. ${ }^{[21]}$ Each derivative was assayed at two concentrations in the presence of the fluorescent indicator. Except for the 6-phenylprotoflavone series (68-73), which showed moderate inhibition at $20 \mu \mathrm{M}$ (14-46\%; see Supporting Information Table 4), none of the compounds inhibited the efflux of calcein AM by P-gp (Figure 4).

\section{Discussion}

This work was initiated with the aim to explore relevant SAR of protoflavones with various substituents at the A-ring and particularly at C6. Following the preparation of acetophenones 41 and 42, a straightforward total-synthetic strategy ${ }^{[22]}$ was applied to obtain 6-substituted protoflavones. It is worth mentioning that, even though related publications typically describe the use of a catalytic amount of iodine for the ring closure to obtain the flavone skeleton, using a larger, one equivalent amount is far more efficient. Structure elucidation of the protoflavones was straightforward based on the mass and ${ }^{1} \mathrm{H}$ NMR spectra. The expected change in the molecular mass,
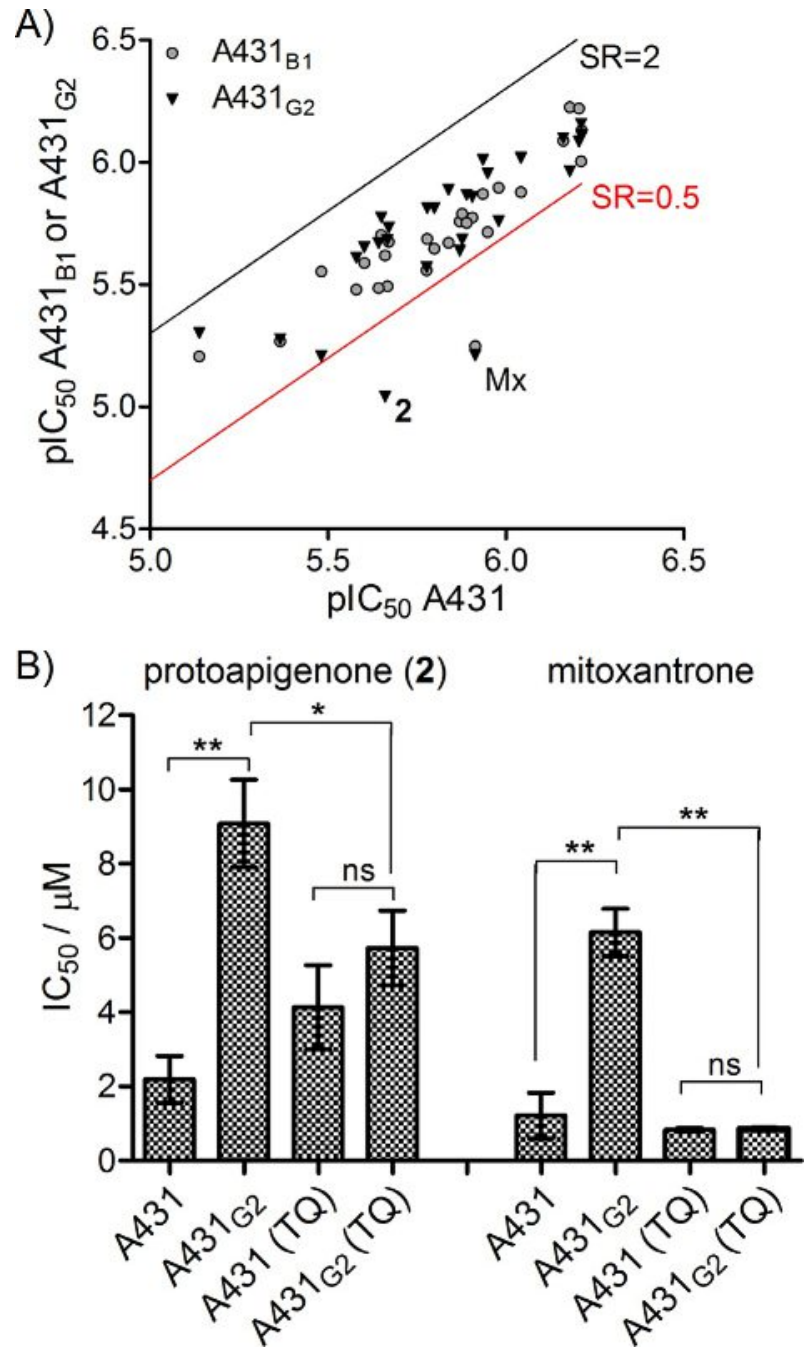

Figure 2. A) Cytotoxic activity of selected protoflavones against $A 431$ (parental) cell line and two MDR derivatives engineered to overexpress $A B C B 1$ $\left(\mathrm{A} 431_{\mathrm{B} 1}\right)$ or $\mathrm{ABCG} 2\left(\mathrm{~A} 431_{\mathrm{G} 2}\right) . \mathrm{SR}=$ selectivity ratio, calculated as $\mathrm{IC}_{50}{ }^{\text {sensitive }}$ / $I_{50}{ }^{M D R} ; n=3-4 ; M x$ : mitoxantrone. $\left.B\right) I C_{50}$ values of 2 (protoapigenone) and mitoxantrone in $A 431$ and $A 431_{\mathrm{G} 2}$ cell lines in the presence and absence of tariquidar (TQ, $1 \mu \mathrm{M}$ ), an ABCG2 efflux inhibitor; ${ }^{*} p<0.05$, ${ }^{* *} p<0.01$.

and, in the case of the $1^{\prime}$-O-alkyl derivatives, the appearance of the characteristic ${ }^{1} \mathrm{H}$ NMR signals and coupling pattern of the side chain proved the successful linking of water or alcohol. The buildup of the protoflavone type B-ring was evidenced by the change in the coupling constant of the two doublets of $\mathrm{H}$ $3^{\prime} / \mathrm{H}-5^{\prime}$ and $\mathrm{H}-2^{\prime} / \mathrm{H}-6^{\prime}$ from $\sim 8.8 \mathrm{~Hz}$ to $\sim 10.0 \mathrm{~Hz}$, together with the remaining $\mathrm{H}-3$ singlet and practically unchanged A-ring signals in the ${ }^{1} \mathrm{H}$ NMR spectrum.

According to their B-ring substitution, cytotoxicity of the protoflavones on the used cell lines typically followed the previously observed SAR: in most cases, 1'-hydroxy-substituted compounds were more toxic than those with 1 '-alkoxy moieties and the isopropyl ethers were the least cytotoxic derivatives. This, however, did not apply for protogenkwanone and its analogues 11-17: protogenkwanone 1'-O-methylether 12 exerted a stronger activity on the mouse lymphoma cells than 11. Moreover, an at least two carbons, long side chain was necessary for this series of compounds to be slightly toxic on 

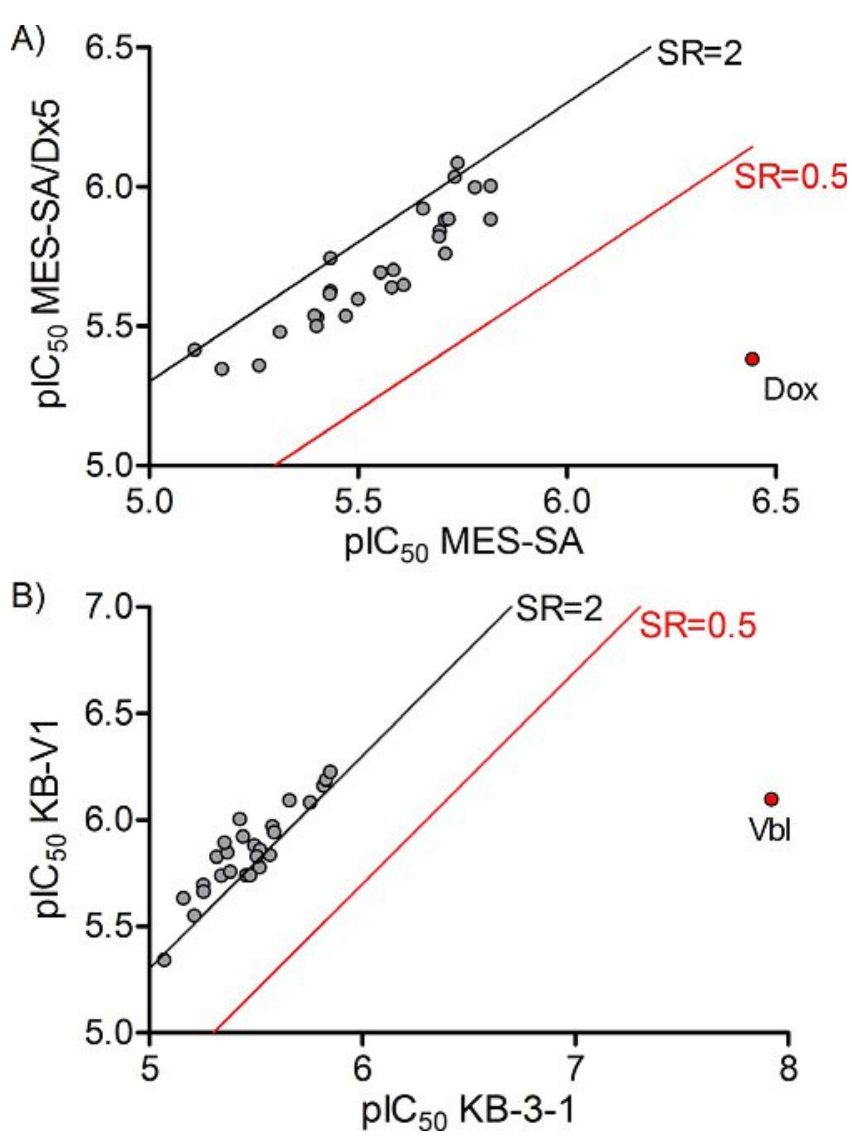

Figure 3. $\mathrm{plC}_{50}$ values measured in doxorubicin-selected MES-SA/Dx $5^{[19]}$ and vinblastine-selected $K B-V 1^{[20]}$ cells relative to the $p \mathrm{C}_{50}$ values of $A$ ) MES-SA and B) KB-3-1 cells. Two compounds were selectively toxic against both MES-SA/Dx5 and KB-V1 cells ( 2 and 66 ), and none of the protoflavones showed substrate-like characteristics; Dox: doxorubicin, Vbl: vinblastine.

MCF-7 cells. The presence of a non-branching propyl or butyl ether side chain at $\mathrm{C}^{\prime}$ ' of protoapigenone (as in compounds 5 and 7) was previously found to be preferable for strong cytotoxic activity. ${ }^{[17]}$ This was also observed in the present study in most cell lines with the exception of the L5178/L5178 $\mathrm{B}_{\mathrm{B} 1}$ and the MCF-7/MCF-7 ${ }_{\text {Dox }}$ cell line pairs. Moreover, the introduction of a 1'-benzyloxy moiety (as in compound 10) to protoapigenone also resulted in an increased toxicity in the $A 431$ cell line and its MDR sub-cell lines, as well as in the KB-3-1 and KB-V1 cells. This provides further evidence for the importance of the size and/or lipophilicity of the substituent at $\mathrm{C}^{\prime}$ and suggests that a larger branching and/or unsaturated alkyl side chain might also lead to an increased cytotoxicity, despite the generally lower activity of the 1 '-O-isopropyl-substituted derivatives than those with linear alkyl chains.

Our attempt to increase the mild selective toxicity of 6-methylprotoflavones $\mathbf{1 8 - 2 4}$ previously observed in ABCB1 transfected L5178 cells ${ }^{[15]}$ by introducing various C 6 substituents revealed one single compound reaching our chosen threshold of relevance against the $\mathrm{L}^{2} 17 \mathrm{~B}_{\mathrm{B} 1}$ cells, 6-methoxyprotoflavone $\mathrm{1}^{\prime}$-O-allylether 29. On the other hand, while all 6-methoxy compounds also showed tendency for such selectivity, other new derivatives showed decreased selective cytotoxicity against this cell line (Table S1). Similarly, none of the tested compounds, including 6-

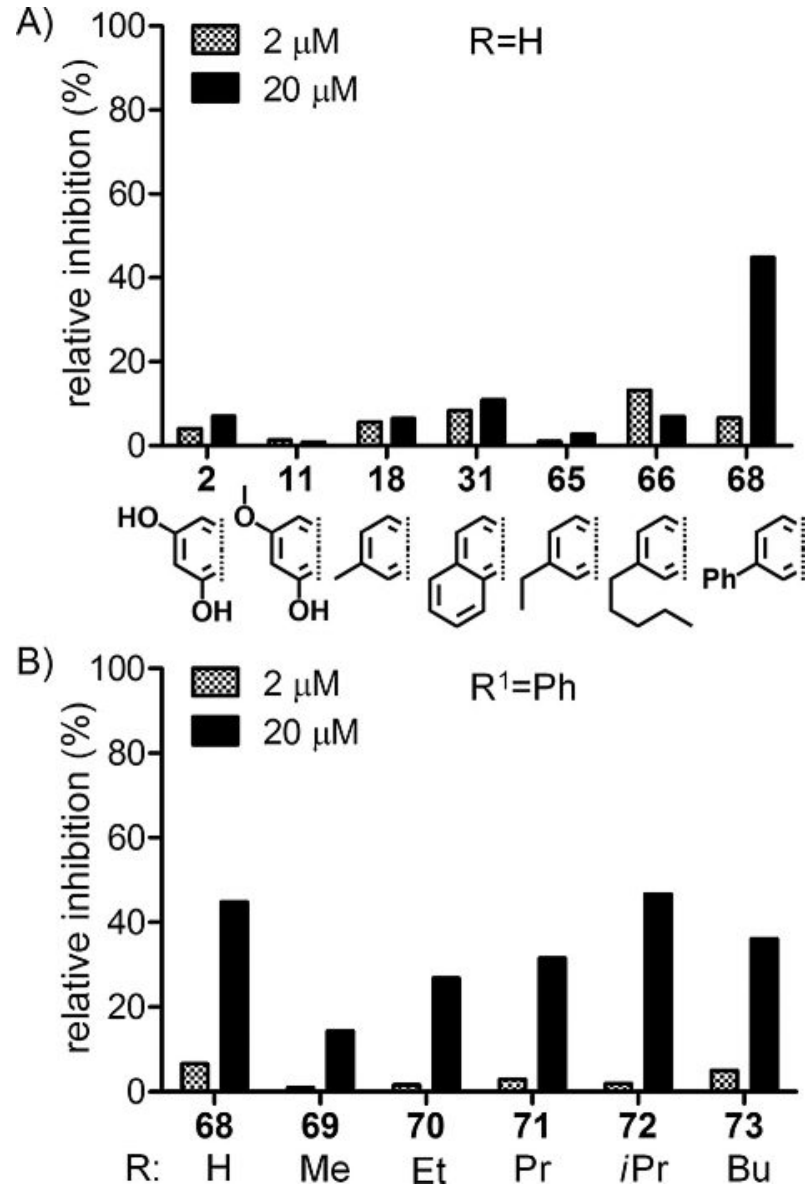

Figure 4. Relative inhibition of calcein AM efflux by A) compounds 2, 11, 18, $31,65,66$, and 68 (where $\mathrm{R}=\mathrm{H}$ refers to Schemes 1 and 2), and $B$ ) derivatives of compound $\mathbf{6 8}$ (6-phenylprotoflavones). Relative inhibition was calculated from mean calcein intensities as: $100^{*}$ [(sample)-(negative control)]/ [(positive control)-(negative control)]; $20 \mu \mathrm{M}$ verapamil was used as positive control, corresponding to full inhibition (100\%); results of single-point experiments are presented.

methylprotoflavone 18 , showed selective toxicity against $A B C B 1$ transfected cell lines, including $A 431_{B 1}$ (Table S2) and MDCK- $\|_{B 1}$ (not shown). Despite the equal toxicity of the studied compounds on parental and P-gp-transfected cell lines, several derivatives proved selectively toxic against MDR cell lines overexpressing P-gp as a result of long-term drug selection. In particular, MCF-7 ${ }_{\text {Dox }}$ cells (adapted to doxorubicin) showed collateral sensitivity to most compounds except for 4, 31, 35 and 59-65, with structural differences of the A-ring clearly influencing activity (Table S1). Compound 68, 6-phenylprotoflavone, for example, showed a remarkable, 13.2-fold selective cytotoxicity, while its $\beta$ naphthoflavone analogue 31, where a fused aromatic ring is connected to the A-ring at the $\mathrm{C} 5 / \mathrm{C} 6$ position, was nonselective. By comparing the selectivity ratios of compounds with different C6 substituents, a clear SAR of the following order was observed: $\mathrm{Ph}>\mathrm{Me}>\mathrm{OMe} \approx$ pentyl, while the ethoxy-substituted protoflavones 59-64 and the ethyl-substituted compound 65 were nonselective. No such general SAR could be concluded for the $\mathrm{C1}^{\prime}$ substituents, except for the lower selectivity observed for the isopropyl ether derivatives 35 and 72. Interestingly, in case of the MES-SA/MES-SA/Dx5 cell line pair where the MDR 
sub-cell line was also obtained by adaptation to doxorubicin, ${ }^{[19]}$ collateral sensitivity was observed only for the classical, 1 '-hydroxy-containing protoflavones (2, 1118 and 66, but SR was below threshold for 31 and 68 ) and not for any of the 1'-O-alkylprotoflavones. Furthermore, the KB-V1 cell line, obtained from $\mathrm{KB}-3-1$ by adaptation to vinblastine ${ }^{[20]}$ also presented marginal CS toward most of the protoflavones, although SR values for several compounds fell just below the 2-fold threshold (Figure 3B, Table S3).

Statistical significance of the SAR was tested from two angles. Compounds were grouped either according to their Arings or their substituents at $\mathrm{C1}^{\prime}$, and the $\mathrm{SR}$ values of these groups were compared by one-way ANOVA ${ }^{1}$ followed by Bonferroni's post hoc test. No differences were observed for the $\mathrm{C}^{\prime}$ ' substituents on any of the cell lines, not even when the data were normalized to the average of their corresponding series (i.e., analogues with the same A-ring). However, the different A-ring-containing protoflavone derivatives showed significant differences in their SR values on the MCF-7/MCF-7 $7_{\text {Dox }}$ cell line pair; results are presented in Figure 5.

All tested compounds were found to be similarly cytotoxic on the $\mathrm{L} 5178$ and the $\mathrm{L}^{2} 17 \mathrm{~B}_{\mathrm{B} 1}$ cell lines, with a strong correlation between the two (Spearman $r=0.9544$ ). Interestingly, $I C_{50}$ values on the MCF-7 $7_{\text {Dox }}$ cell line also showed a good correlation to those on the parental mouse lymphoma cell line (Spearman $r=0.7691$ ), while the same correlation for MCF-7 was much weaker with several outliers (Spearman $r=0.5812$; without outliers, that is, compounds 8, 11-17, 19-24, 37, 68-71 and 73: $r=0.7420$ ). For a graphical interpretation of these correlations, see Supporting Information Figure S1.

These results suggest that the SR associated with the compounds on the MCF-7/MCF-7 ${ }_{\text {Dox }}$ is more a result of the resistance of MCF-7 to protoflavones, than the sensitivity of MCF$7_{\text {Dox }}$ MCF-7 cells appear to be particularly resistant to the 7methoxy group containing protogenkwanone derivatives 1117, 6-methylprotoflavones 19-24, 6-phenylprotoflavones 6871 and 73, and 1'-allyl-group-containing analogues 8 and 37, in line with the results presented in Figure S1. As such, adaptation of MCF-7 cells to doxorubicin has apparently resulted in the loss of initial resistance to protoflavones as an evolutionary cost of acquiring the MDR phenotype, and this manifested as collateral sensitivity.

Collateral sensitivity is causally linked to the adaption of MDR cells to a chemotherapeutic and may involve metabolic modifications, the upregulation of receptors ${ }^{[23]}$ or the modulation of the redox homeostasis. ${ }^{[24]}$ One limitation of studies relying on MDR cell lines is that the contribution of MDR pumps, versus other acquired cellular alterations, cannot be delineated. ${ }^{[8,9,25]}$ Collateral sensitivity of the MDR cell lines analyzed in this study indicates that resistance to doxorubicin or vinblastine may result in cellular alterations that render the cells susceptible to the protoflavone derivatives. However, in contrast

${ }^{1}$ Each group containing at least seven data points passed the Shapiro-Wilk normality test, suggesting the normal distribution of SR values under the influence of the presented chemical variations. Therefore, ANOVA is suitable for the statistical evaluation of these datasets.

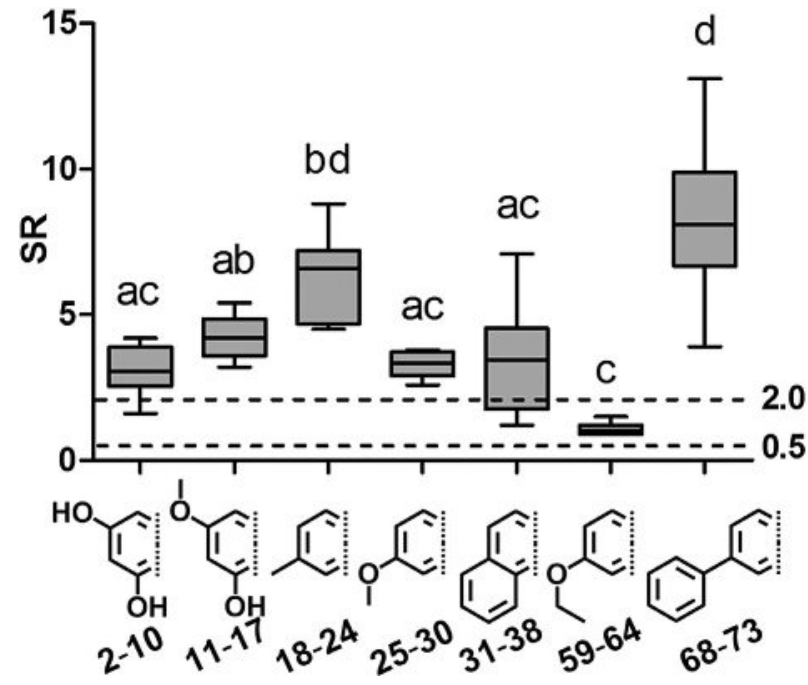

Figure 5. Selectivity ratio (SR) values for protoflavone analogues containing the same A-rings on the MCF-7/MCF-7 ${ }_{\text {Dox }}$ cell line pair. Box-and-whisker plots represent medians, first and third quartiles and ranges; $S R \geq 2.0$ and $S R \leq 0.5$ represent $C S$ and $C R$, respectively; lower-case letters represent datasets with statistically significant differences (i.e., groups with overlap in their marking are not significantly different) at $p \leq 0.05$ by one-way ANOVA followed by Bonferroni's post-hoc test.

to MDR-selective compounds, ${ }^{[10]}$ protoflavone derivatives do not selectively target cells engineered to overexpress P-gp, suggesting that the increased toxicity observed in the MDR cells is not conferred by the efflux pumps. This was also supported by our observation that selectivity ratios did not decrease significantly when compounds 2, 11, and 18 were tested on the MES-SA/MES-SA/Dx5 cell line pair in the presence of tariquidar (data not presented).

It is important to point out that the lack of CR to most protoflavones in all MDR cell lines studied here indicates that these compounds can overcome MDR through bypassing efflux that is mediated by $A B C B 1$ or $A B C G 2$. SAR of the cytotoxic activity concerning the 6-substituents appears to differ from cell line to cell line, for example an order of $\mathrm{Me}>\mathrm{Et} \approx$ Pent $\approx \mathrm{Ph}>\mathrm{Br}>\mathrm{OEt}>$ OMe can be recognized on $\mathrm{L} 5178$ and $\mathrm{L}^{2} 178_{\mathrm{B} 1}$, Pent $>\mathrm{Ph} \approx \mathrm{Me}$ on KB-3-1 and KB-V1, while similar activities are exerted by 6-pentyl (66), 6-phenyl (68) and 6methyl (18) compounds on MES-SA and MES-SA/Dx5. From a general overview, 6-alkyl-substituted protoflavones appear to be somewhat more favorable anticancer agents over 6-alkoxy compounds, even though nearly all compounds presented here can be considered as valuable leads against resistant cancers. As an interesting exception to this, however, resistance to protoapigenone (2) was observed in the ABCG2 transfected $\mathrm{A} 431_{\mathrm{G} 2}$ cell line, and a tendency for marginal resistance appeared also to its $1^{\prime}$-O-alkyl ethers. Resistance of this cell line to protoapigenone (2) markedly decreased in the presence of tariquidar, strongly suggesting that compound $\mathbf{2}$ is an ABCG2 substrate. $A B C G 2$ did not confer resistance toward any of the other compounds including protogenkwanone (11), which differs from 2 only in its 7-methoxy group. This suggests that a nonsubstituted phenolic hydroxy group at C7 is necessary for protoflavones to be recognized by this transporter. 


\section{Conclusions}

Our in vitro studies on various A-ring and 1'-substituted protoflavones revealed 6-methoxyprotoflavone 1'-O-allyl ether 29 as an antitumor agent with a mild MDR selectivity $(S R=2.0)$ in a murine lymphoma cell line transfected with the human $A B C B 1$ efflux transporter.

The ability of protoflavones to evade efflux-mediated MDR was confirmed both in $A B C B 1$ and $A B C G 2$ expressing cell lines, with the exceptions of protoapigenone (2) which was identified as an ABCG2 substrate. MDR selective cytotoxicity was observed for most of the tested protoflavones in a breast cancer cell line adapted to doxorubicin (MCF- $7_{\text {Dox }}$ ) and SAR revealed importance of the A-ring substitution, while in the uterine sarcoma MES-SA/Dx5, another doxorubicin-selected cell line, only the 1'-hydroxy-containing compounds showed relevant selectivity. Because overexpression of $A B C B 1$ did not sensitize cells, we conclude that the MDR-selective cytotoxicity of protoflavones is connected to other changes accompanying acquired drug resistance.

\section{Experimental Section}

Structure elucidation was carried out by NMR spectroscopy and MS. NMR spectra were obtained on a Varian Gemini-2000 $200 \mathrm{MHz}$ or Bruker Avance DRX-500 NMR spectrometer in $\mathrm{CDCl}_{3}, \mathrm{CD}_{3} \mathrm{OD}$, $\left[D_{6}\right]$ acetone, or $\left[D_{6}\right]$ DMSO. Mass spectra were taken on an API 2000 triple-quadrupole (Ab Sciex, USA) or LCMS-IT-TOF (Shimadzu, Japan) with an ESI interface. Compounds were purified by rotation planar chromatography on a chromatotron (Harrison Research, USA) with adequately chosen eluents of $n$-hexane/EtOAc on silica $\mathrm{GF}_{254}$ (Merck, Germany), or by flash chromatography on a Combiflash $R_{\mathrm{f}}+$ (Teledyne Isco, USA) with eluents of $n$-hexane/EtOAc or $\mathrm{MeOH} / \mathrm{CH}_{2} \mathrm{Cl}_{2}$ on RediSep normal-phase silica flash columns (Teledyne Isco, USA). All compounds possessed a purity of $\geq 95.0 \%$ as determined by HPLC-DAD, except for compounds $\mathbf{5 1}$ and $\mathbf{5 5}$ (92.20 and $93.41 \%$, respectively), which served as intermediates for further synthesis and whose bioactivity was not tested. All chemicals were obtained from Aldrich Inc. (USA).

Synthesis of 5 '-ethyl- and 5'-pentyl-2'-hydroxyacetophenone (41 and 42): In the first step, 4-ethyl- and 4-pentylphenol acetate (39 and 40 , respectively) were synthesized by adding acetic anhydride $(0.1 \mathrm{~mol}, 10.2 \mathrm{~g})$ and one drop of $\mathrm{CCH}_{2} \mathrm{SO}_{4}$ to 4-ethyl or 4-pentylphenol $(0.1 \mathrm{~mol})$ and stirring at room temperature (RT) for $20 \mathrm{~min}$. The mixture was then poured into $\mathrm{H}_{2} \mathrm{O}$ and extracted with EtOAc $(3 \times 50 \mathrm{~mL})$. The organic layer was evaporated under reduced pressure, re-dissolved in $\mathrm{CH}_{2} \mathrm{Cl}_{2}$ and crystallized anhydrous $\mathrm{AlCl}_{3}$ was added little by little under ice bed cooling. The mixture was held at reflux for $10 \mathrm{~h}$, then the reaction was stopped by adding crushed ice. After filtration, the precipitate was purified on silica to obtain $\mathbf{4 1}$ or $\mathbf{4 2 .}$

\section{General procedure for chalcone synthesis}

The 5'-substituted 2'-hydroxyacetophenone (41-44) and 4-benzyloxybenzaldehyde $(3.0 \mathrm{~g}, 14.3 \mathrm{mmol})$ were dissolved in $50 \% \mathrm{EtOH}$, $\mathrm{KOH} / \mathrm{H}_{2} \mathrm{O}$ solution $(20 \mathrm{~mL})$. The reaction was stirred at RT for $30 \mathrm{~h}$, and then the solvent was evaporated under reduced pressure. The mixture was purified on silica gel (isocratic elution, n-hexane/ EtOAc, 6:1) to afford $\mathbf{4 5 - 4 8 .}$
(E)-3-(4-benzyloxyphenyl)-1-(5-ethoxy-2-hydroxyphenyl)prop-2en-1-one (47): Orange solid; yield: $82.2 \%$; NP-HPLC purity: 99.15\%; ${ }^{1} \mathrm{H}$ NMR $\left(500 \mathrm{MHz}, \mathrm{CDCl}_{3}\right) \delta=12.47(1 \mathrm{H}, \mathrm{s}, \mathrm{OH}), 7.89(1 \mathrm{H}$, $\mathrm{d}, J=15.35 \mathrm{~Hz}), 7.62(2 \mathrm{H}, \mathrm{d}, J=8.60 \mathrm{~Hz}), 7.47(1 \mathrm{H}, \mathrm{d}, J=15.55 \mathrm{~Hz})$, $7.34-7.45(6 \mathrm{H}, \mathrm{m}), 7.12(1 \mathrm{H}, \mathrm{dd}, J=9.00 \mathrm{~Hz}, 2.80 \mathrm{~Hz}), 7.02(2 \mathrm{H}, \mathrm{d}$, $J=8.55 \mathrm{~Hz}), 6.96(1 \mathrm{H}, \mathrm{d}, J=9.00 \mathrm{~Hz}), 5.13(2 \mathrm{H}, \mathrm{s}), 4.05(2 \mathrm{H}, \mathrm{q}, J=$ $6.95 \mathrm{~Hz}), 1.44(3 \mathrm{H}, \mathrm{t}, J=6.95 \mathrm{~Hz}) 7.91(2 \mathrm{H}, \mathrm{d}, J=8.45 \mathrm{~Hz}), 7.86(1 \mathrm{H}$, $\mathrm{d}, J=\mathrm{Hz}), 7.67(1 \mathrm{H}, \mathrm{d}, J=2.65 \mathrm{~Hz}), 7.4-7.38(5 \mathrm{H}, \mathrm{br}), 7.35(1 \mathrm{H}, \mathrm{d}$, $J=7.25 \mathrm{~Hz}), 7.19(1 \mathrm{H}, \mathrm{dd}, J=2.70 \mathrm{~Hz}, 8.50 \mathrm{~Hz}), 7.11(2 \mathrm{H}, \mathrm{d}, J=$ $8.50 \mathrm{~Hz}), 6.92(1 \mathrm{H}, \mathrm{d}, J=9.00 \mathrm{~Hz}), 5.19(2 \mathrm{H}, \mathrm{s}), 4.07(2 \mathrm{H}, \mathrm{q}, J=$ $6.85 \mathrm{~Hz}), 1.34 \mathrm{ppm}(3 \mathrm{H}, \mathrm{t}, J=6.90 \mathrm{~Hz}) ;{ }^{13} \mathrm{C}$ NMR $\left(125 \mathrm{MHz}, \mathrm{CDCl}_{3}\right)$ $193.45,161.32,157.97,151.11,145.47,136.46,130.69,128.83$, $128.36,127.72,127.61,124.29,119.97,119.31,117.93,115.53$, 114.22, 70.29, 64,72, 15.06; ESIMS (m/z): $375.3[\mathrm{M}+\mathrm{H}]^{+}$.

\section{General procedure for flavone synthesis}

Chalcone (3 mmol, 45-48) was dissolved in DMSO $(5 \mathrm{~mL})$, and iodine (1 equiv., $76 \mathrm{mg}, 0.3 \mathrm{mmol}$ ) was added. The solution was stirred and heated at $110^{\circ} \mathrm{C}$. After $2 \mathrm{~h}, \mathrm{Na}_{2} \mathrm{~S}_{2} \mathrm{O}_{3}(10 \%, 50 \mathrm{~mL})$ was added to remove the iodine. The mixture was extracted with EtOAc $(3 \times 50 \mathrm{~mL})$ and then purified by column chromatography on silica gel to afford compounds 49-52.

6-Ethoxy-4'-benzyloxyflavone (51): Yield: $85.2 \%$; NP-HPLC purity: $92.20 \%$, pale-yellow solid; ${ }^{1} \mathrm{H}$ NMR $\left(500 \mathrm{MHz}, \mathrm{CDCl}_{3}\right) \delta=7.86(2 \mathrm{H}$, $\mathrm{d}, J=8.80 \mathrm{~Hz}), 7.56(1 \mathrm{H}, \mathrm{d}, J=2.70 \mathrm{~Hz}), 7.48-7.38(5 \mathrm{H}$, benzyl), 7.35 $(1 \mathrm{H}, \mathrm{d}, J=7.1 \mathrm{~Hz}), 7.25(1 \mathrm{H}, \mathrm{dd}, J=2.85 \mathrm{~Hz}, 8.75 \mathrm{~Hz}), 7.08(2 \mathrm{H}, \mathrm{d}$, $J=8.70 \mathrm{~Hz}), 6.72(1 \mathrm{H}, \mathrm{s}), 5.14(2 \mathrm{H}, \mathrm{s}), 4.13(2 \mathrm{H}, \mathrm{q}, J=6.95 \mathrm{~Hz})$, $1.44 \mathrm{ppm}(3 \mathrm{H}, \mathrm{t}, J=6.95 \mathrm{~Hz}) ;{ }^{13} \mathrm{C}$ NMR $\left(125 \mathrm{MHz}, \mathrm{CDCl}_{3}\right)$ 178.44, $163.26,161.61,156.43,151.06,136.34,131.02,128.95,128.87$, $128.41,128.11,127.62,124.68,124.61,124.05,119.46,115.48$, 105.72, 70.36, 64.36, 14.86 ppm; ESIMS (m/z): $372.9[\mathrm{M}+\mathrm{H}]^{+}$.

\section{Synthesis of compound 53 from 52 via Suzuki coupling}

Compound $52(407.26 \mathrm{mg}, 1.0 \mathrm{mmol})$, phenylboronic acid (1.2 equiv., $146.3 \mathrm{mg}, 1.2 \mathrm{mmol}$ ) and $\mathrm{K}_{2} \mathrm{CO}_{3}$ (2 equiv., $276.42 \mathrm{mg}$, $2.0 \mathrm{mmol}$ ) were dissolved in $\mathrm{H}_{2} \mathrm{O}(7 \mathrm{~mL})$, and propanol $(25 \mathrm{~mL})$ and tetrakis(triphenylphosphine)palladium $(0) \quad(5 \mathrm{mmol})$ were subsequently added. The reaction was performed under $\mathrm{N}_{2}$ gas at $40^{\circ} \mathrm{C}$, to afford $\mathbf{5 3}$ as a yellow solid.

6-Phenyl-4'-benzyloxyflavone (53): Yield: $30.0 \%$; yellow solid; ${ }^{1} \mathrm{H}$ NMR $\left(200 \mathrm{MHz}, \mathrm{CDCl}_{3}\right) \delta=8.45(1 \mathrm{H}, \mathrm{s}), 7.89(3 \mathrm{H}, \mathrm{d}, J=7.80 \mathrm{~Hz})$, $7.67(1 \mathrm{H}, \mathrm{d}, J=7.20 \mathrm{~Hz}), 7.50-7.30(11 \mathrm{H}, \mathrm{m}), 7.25(1 \mathrm{H}, \mathrm{s}), 7.10(2 \mathrm{H}$, $\mathrm{d}, J=8.40 \mathrm{~Hz}), 6.77(1 \mathrm{H}, \mathrm{s}), 5.15 \mathrm{ppm}(2 \mathrm{H}, \mathrm{s}) ;{ }^{13} \mathrm{C}$ NMR $(50 \mathrm{MHz}$, $\mathrm{CDCl}_{3}$ ) 177.35, 162.34, 160.55, 154.56, 138.32, 137.20, 135.16, $131.35,127.91,127.68,127.35,127.21,127.00,126.74,126.41$, $126.12,125.66,123.19,122.48,117.39,114.33,113.90,105.18,97.23$, 69.17.

\section{General procedure for benzyl group removal}

A mixture of 4'-benzyloxyflavone $(0.5 \mathrm{mmol}, 49-53)$, dry $\mathrm{Pd} / \mathrm{C}$ $(10 \%, 106 \mathrm{mg})$, and EtOAc $(20 \mathrm{~mL})$ was stirred at $25^{\circ} \mathrm{C}$ under an atmosphere of hydrogen for $10 \mathrm{~h}$. The mixture was filtered, washed with EtOAc, concentrated under vacuum and purified by column chromatography on silica gel to afford compounds 54-58.

6-Pentyl-4'-hydroxyflavone (55): Yield: $85.4 \%$; NP-HPLC purity: $93.41 \%$, pale-yellow solid; ${ }^{1} \mathrm{H}$ NMR $\left(500 \mathrm{MHz},\left[\mathrm{D}_{6}\right] \mathrm{DMSO}\right) \delta=10.31$ $(1 \mathrm{H}, \mathrm{brs}, \mathrm{OH}), 7.95(2 \mathrm{H}, \mathrm{d}, J=8.65 \mathrm{~Hz}), 7.81(1 \mathrm{H}, \mathrm{s}), 7.66(1 \mathrm{H}, \mathrm{d}, J=$ $8.45 \mathrm{~Hz}), 7.64(1 \mathrm{H}, \mathrm{dd}, J=8.85 \mathrm{~Hz}, 1.85 \mathrm{~Hz}), 6.90(2 \mathrm{H}, \mathrm{d}, J=9.75 \mathrm{~Hz})$, 
$6.94(2 \mathrm{H}, \mathrm{d}, J=8.60 \mathrm{~Hz}), 6.84(1 \mathrm{H}, \mathrm{s}), 2.70(2 \mathrm{H}, \mathrm{t}, J=7.45 \mathrm{~Hz}), 1.61$ $(2 \mathrm{H}$, quin, $J=7.00 \mathrm{~Hz}), 1.38-1.21(4 \mathrm{H}, \mathrm{m}), 0.85 \mathrm{ppm}(2 \mathrm{H}, \mathrm{t}, J=$ $6.85 \mathrm{~Hz}) ;{ }^{13} \mathrm{C}$ NMR $(125 \mathrm{MHz}$, [D $]$ DMSO $) 176.97,162.97,160.96$, $154.05,139.67,134.43,128.34,123.43,123.07,121.7,118.26,115.97$, 104.72, 34.42, 30.79, 30.55, 21.96, 13.94; ESIMS (m/z): $309.5[\mathrm{M}+\mathrm{H}]^{+}$.

\section{General procedure for protoflavone synthesis from 4'- hydroxyflavones}

Apigenin, genkwanin, 4'-hydroxy-6-methylflavone, 4'-hydroxy-6methoxyflavone, 4'-hydroxy- $\beta$-naphthoflavone (Indofine, Hillsborough, NJ, USA), or the synthesized 4 '-hydroxyflavone $\left(1 \mathrm{mg} \mathrm{mL}^{-1}\right.$, 54-58) was dissolved in a mixture of acetonitrile and $\mathrm{H}_{2} \mathrm{O}(9: 1 \mathrm{v} / \mathrm{v}$ ratio) or the alcohol to be coupled at position $\mathrm{C}^{\prime}$. Two equivalents of [bis(trifluoroacetoxy)iodo]benzene were added to the mixture. After stirring at $80^{\circ} \mathrm{C}$ for $1 \mathrm{~h}$, the mixture was cooled down, evaporated under reduced pressure and purified by flash chromatography to obtain compounds $2-9,{ }^{[17]} 10,11-24,,^{[15]} 25-30,31-38^{[17]}$ or 59-73. Compounds $10,25-30$ and 59-73 are reported here as new protoflavones.

Protoapigenone 1'-O-benzyl ether (10): Light-brown solid; yield: 39,6\%; RP-HPLC purity: $99.21 \%,{ }^{1} \mathrm{H}$ NMR $\left(500 \mathrm{MHz},\left[\mathrm{D}_{6}\right] \mathrm{DMSO}\right) \delta=$ $12.47(1 \mathrm{H}, \mathrm{s}, \mathrm{OH}), 7.36-7.43(4 \mathrm{H}, \mathrm{m}), 7.32(1 \mathrm{H}, \mathrm{t}, J=6.90 \mathrm{~Hz}), 7.18$ $(2 \mathrm{H}, \mathrm{d}, J=9.95 \mathrm{~Hz}), 6.57(2 \mathrm{H}, \mathrm{d}, J=9.95 \mathrm{~Hz}), 6.50(1 \mathrm{H}, \mathrm{s}), 6.18(2 \mathrm{H}$, $\mathrm{d}, J=9.15 \mathrm{~Hz}), 4.57 \mathrm{ppm}(2 \mathrm{H}, \mathrm{s}) ;{ }^{13} \mathrm{C}$ NMR $\left(125 \mathrm{MHz},\left[\mathrm{D}_{6}\right] \mathrm{DMSO}\right)$ 184.29, 181.32, 165.44, 164.37, 161.41, 157.41, 145.78, 137.53, $132.22,128.35,127.84,127.65,107.37,103.68,99.43,94.01,74.22$, 66.52; $\operatorname{ESIMS~}(\mathrm{m} / \mathrm{z}): 377.2[\mathrm{M}+\mathrm{H}]^{+}$.

6-Methoxyprotoflavone (25): Pale-yellow solid; yield: 32.3\%; NPHPLC purity: $98.95 \%,{ }^{1} \mathrm{H}$ NMR (500 MHz, [D $]$ ]acetone) $\delta=7.46(1 \mathrm{H}$, $\mathrm{d}, J=2.95 \mathrm{~Hz}), 7.44(1 \mathrm{H}, \mathrm{d}, J=9.35 \mathrm{~Hz}), 7.33(1 \mathrm{H}, \mathrm{dd}, J=9.07 \mathrm{~Hz}$, $2.90 \mathrm{~Hz}), 7.05(2 \mathrm{H}, \mathrm{d}, J=10.00 \mathrm{~Hz}), 6.66(1 \mathrm{H}, \mathrm{s}), 6.33(2 \mathrm{H}, \mathrm{d}, J=$ $10.00 \mathrm{~Hz}), 6.11(1 \mathrm{H}, \mathrm{s}), 3.90(3 \mathrm{H}, \mathrm{s}) ;{ }^{13} \mathrm{C} \mathrm{NMR}\left(125 \mathrm{MHz}, \mathrm{CDCl}_{3}\right)$ $185.54,179.13,166.64,157.4,151.23,147.00,129.71,124.57,119.68$, 107.97, 104.84, 69.85, 56.09; ESIMS $(m / z): 285.4\left[M+\mathrm{H}^{+}\right.$.

6-Methoxyprotoflavone 1'-O-methyl ether (26): Yellow solid; yield: 41.3\%; NP-HPLC purity: $98.93 \%,{ }^{1} \mathrm{H} N M R \quad(500 \mathrm{MHz}$, $\left[\mathrm{D}_{6}\right.$ ]acetone $) \delta=7.45(1 \mathrm{H}, \mathrm{d}, J=3.20 \mathrm{~Hz}), 7.43(1 \mathrm{H}, \mathrm{d}, J=8.7 \mathrm{~Hz})$, $7.33(1 \mathrm{H}, \mathrm{dd}, J=9.15 \mathrm{~Hz}, 3.00 \mathrm{~Hz}), 7.01(2 \mathrm{H}, \mathrm{d}, J=10.05 \mathrm{~Hz}), 6.58$ $(1 \mathrm{H}, \mathrm{s}), 6.54(2 \mathrm{H}, \mathrm{d}, J=10.15 \mathrm{~Hz}), 3.90(3 \mathrm{H}, \mathrm{s}), 3.44 \mathrm{ppm}(3 \mathrm{H}, \mathrm{s})$; ${ }^{13} \mathrm{C}$ NMR $\quad(125 \mathrm{MHz}$ ，[D $]$ DMSO) 184.27, 176.53, 163.86, 156.74, $150.26,145.91,132.42,123.96,123.53,119.89,108,104.77,74.3$, 55.74, 52.34; $\operatorname{ESIMS~(m/z):~} 299.1[\mathrm{M}+\mathrm{H}]^{+}$.

6-Methoxyprotoflavone 1'-O-ethyl ether (27): Yellow solid; yield: $39.3 \%$; NP-HPLC purity: $98.39 \%,{ }^{1} \mathrm{H}$ NMR $\left(500 \mathrm{MHz},\left[\mathrm{D}_{6}\right.\right.$ ]acetone $)$ $\delta=7.45(1 \mathrm{H}, \mathrm{d}, J=2.90 \mathrm{~Hz}), 7.43(1 \mathrm{H}, \mathrm{d}, J=8.85 \mathrm{~Hz}), 7.33(1 \mathrm{H}, \mathrm{dd}$, $J=10.00,2.80 \mathrm{~Hz}), 7.03(2 \mathrm{H}, \mathrm{d}, J=10.00 \mathrm{~Hz}), 6.63(1 \mathrm{H}, \mathrm{s}), 6.51(2 \mathrm{H}$, $\mathrm{d}, J=9.95 \mathrm{~Hz}), 3.90(3 \mathrm{H}, \mathrm{s}), 3.64(2 \mathrm{H}, \mathrm{q}, J=6.80 \mathrm{~Hz}) 1.28 \mathrm{ppm}(1 \mathrm{H}$, $\mathrm{t}, J=6.90 \mathrm{~Hz}) ;{ }^{13} \mathrm{C}$ NMR $\quad\left(125 \mathrm{MHz}, \quad\left[\mathrm{D}_{6}\right] \mathrm{DMSO}\right) 184.34,176.55$, $164.04,156.73,150.24,146.39,131.91,123.96,123.5,119.88,107.96$, 104.78, 74.01, 60.3, 55.73, 15.56; ESIMS (m/z): $313.0[\mathrm{M}+\mathrm{H}]^{+}$.

6-Methoxyprotoflavone 1'-O-butyl ether (28): Yield: 46.3\%; NPHPLC purity: $98.67 \%$, light-brown solid; ${ }^{1} \mathrm{H} \mathrm{NMR}(500 \mathrm{MHz}$, $\left[\mathrm{D}_{6}\right.$ ]acetone) $\delta=7.45(1 \mathrm{H}, \mathrm{d}, J=2.90 \mathrm{~Hz}), 7.43(1 \mathrm{H}, \mathrm{d}, J=8.80 \mathrm{~Hz})$, $7.32(1 \mathrm{H}, \mathrm{dd}, J=9.15 \mathrm{~Hz}, 1.80 \mathrm{~Hz}), 7.02(2 \mathrm{H}, \mathrm{d}, J=10.25 \mathrm{~Hz}), 6.63$ $(1 \mathrm{H}, \mathrm{s}), 6.51(2 \mathrm{H}, \mathrm{d}, J=9.85 \mathrm{~Hz}), 3.90(3 \mathrm{H}, \mathrm{s}), 3.59(2 \mathrm{H}, \mathrm{t}, J=$ $5.35 \mathrm{~Hz}) 1.65(2 \mathrm{H}, \mathrm{q}, J=6.20 \mathrm{~Hz}), 1.51-1.35(2 \mathrm{H}, \mathrm{m}), 0.94 \mathrm{ppm}(3 \mathrm{H}$, $\mathrm{t}, J=7.20 \mathrm{~Hz}) ;{ }^{13} \mathrm{C}$ NMR $\left(125 \mathrm{MHz}, \mathrm{CDCl}_{3}\right) 184.93,178.42,164.14$, $153.28,149.20,146.45,132.77,124.66,124.25,119.64,108.89$,
104.99, 74.65, 65.09, 56.11, 32.23, 19.43, 13.99; ESIMS (m/z): 341.1 $[\mathrm{M}+\mathrm{H}]^{+}$

6-Methoxyprotoflavone 1'-O-allyl ether (29): Light-brown solid; yield: $37.2 \%$; NP-HPLC purity: $95.17 \%,{ }^{1} \mathrm{HNMR} \quad(500 \mathrm{MHz}$, $\left[\mathrm{D}_{6}\right.$ ]acetone $) \delta=7.44(1 \mathrm{H}, \mathrm{d}, J=2.85 \mathrm{~Hz}), 7.43(1 \mathrm{H}, \mathrm{d}, J=8.85 \mathrm{~Hz})$, $7.30(1 \mathrm{H}, \mathrm{dd}, J=9.15 \mathrm{~Hz}, 2.95 \mathrm{~Hz}), 7.02(2 \mathrm{H}, \mathrm{d}, J=10.10 \mathrm{~Hz}), 6.61$ $(1 \mathrm{H}, \mathrm{s}), 6.49(2 \mathrm{H}, \mathrm{d}, J=9.85 \mathrm{~Hz}), 5.24(1 \mathrm{H}$, octet, $J=6.55 \mathrm{~Hz}), 4.61$ $(1 \mathrm{H}, \mathrm{d}, J=17.2 \mathrm{~Hz}), 4.43(1 \mathrm{H}, \mathrm{d}, J=10.57 \mathrm{~Hz}), 3.37(2 \mathrm{H}, \mathrm{d}, J=$ $5.05 \mathrm{~Hz}), 3.86 \mathrm{ppm}(3 \mathrm{H}, \mathrm{s}) ;{ }^{13} \mathrm{C} \mathrm{NMR}\left(125 \mathrm{MHz}, \mathrm{CDCl}_{3}\right)$ 184.69, $178.25,163.8,157.37,151.09,145.81,145.78,133.75,132.87$, 124.24, 123.68, 119.6, 117.69, 108.98, 105.01, 74.83, 66.23, 56.09; $\operatorname{ESIMS}(\mathrm{m} / \mathrm{z}): 325.2[\mathrm{M}+\mathrm{H}]^{+}$.

6-Methoxyprotoflavone 1'-O-proargyl ether (30): Light-brown solid; yield: $37.7 \%$; NP-HPLC purity: $95.32 \%,{ }^{1} \mathrm{H}$ NMR $(500 \mathrm{MHz}$, $\left[\mathrm{D}_{6}\right.$ ]acetone) $\delta=7.46(1 \mathrm{H}, \mathrm{d}, J=2.90 \mathrm{~Hz}), 7.44(1 \mathrm{H}, \mathrm{d}, J=8.85 \mathrm{~Hz})$, $7.33(1 \mathrm{H}, \mathrm{dd}, J=9.07 \mathrm{~Hz}, 2.80 \mathrm{~Hz}), 7.10(2 \mathrm{H}, \mathrm{d}, J=9.90 \mathrm{~Hz}), 6.61$ $(1 \mathrm{H}, \mathrm{s}), 6.50(2 \mathrm{H}, \mathrm{d}, J=9.90 \mathrm{~Hz}), 4.44(2 \mathrm{H}, \mathrm{d}, J=1.95 \mathrm{~Hz}), 3.90(3 \mathrm{H}$, s), $3.10 \mathrm{ppm}(1 \mathrm{H}, \mathrm{t}, J=2.20 \mathrm{~Hz}) ;{ }^{13} \mathrm{C}$ NMR $\left(125 \mathrm{MHz},\left[\mathrm{D}_{6}\right] \mathrm{DMSO}\right)$ $184.17,176.5,163.24,156.76,150.27,144.90,132.26,123.95$, 123.57, 119.92, 108.17, 104.76, 80.17, 78.41, 74.40, 55.74, 53.27; $\operatorname{ESIMS~(m/z):~} 322.9[\mathrm{M}+\mathrm{H}]^{+}$.

6-Ethoxyprotoflavone (59): Pale-yellow solid; yield: $35.3 \%$; NPHPLC purity: $99.14 \%,{ }^{1} \mathrm{H} N M R\left(500 \mathrm{MHz}, \mathrm{CD}_{3} \mathrm{OD}\right) \delta=7.51(1 \mathrm{H}, \mathrm{d}$, $J=2.90 \mathrm{~Hz}), 7.43(1 \mathrm{H}, \mathrm{d}, J=9.20 \mathrm{~Hz}), 7.36(1 \mathrm{H}, \mathrm{dd}, J=9.25 \mathrm{~Hz}$, $3.00 \mathrm{~Hz}), 7,00(2 \mathrm{H}, \mathrm{d}, J=9.95 \mathrm{~Hz}), 6.79(1 \mathrm{H}, \mathrm{s}), 6.39(2 \mathrm{H}, \mathrm{d}, J=$ $10.10 \mathrm{~Hz}), 4.14(2 \mathrm{H}, \mathrm{q}, J=6.95 \mathrm{~Hz}), 1.44 \mathrm{ppm}(3 \mathrm{H}, \mathrm{t}, J=7.00 \mathrm{~Hz})$; ${ }^{13} \mathrm{C}$ NMR $\left(125 \mathrm{MHz}, \mathrm{CDCl}_{3}\right)$ 185.37, 178.99, 166.3, 156.73, 151.08, 146.77, 129.75, 124.79, 124.23, 119.58, 107.97, 105.45, 69.78, 64.40, 14.72; $\operatorname{ESIMS~(m/z):~} 299.3\left[M+\mathrm{H}^{+}\right.$

6-Ethoxyprotoflavone 1'-O-methyl ether (60): Yellow solid; yield: $42.0 \%$; NP-HPLC purity: $99.21 \%$, ${ }^{1} \mathrm{H}$ NMR $\left(500 \mathrm{MHz}, \mathrm{CDCl}_{3}\right) \delta=7.48$ $(1 \mathrm{H}, \mathrm{d}, J=2.90 \mathrm{~Hz}), 7.26(1 \mathrm{H}, \mathrm{d}, J=9.15 \mathrm{~Hz}), 7.20(1 \mathrm{H}, \mathrm{dd}, J=$ $9.15 \mathrm{~Hz}, 2.90 \mathrm{~Hz}), 6.79(2 \mathrm{H}, \mathrm{d}, J=10.00 \mathrm{~Hz}), 6.71(1 \mathrm{H}, \mathrm{s}), 6.54(2 \mathrm{H}, \mathrm{d}$, $J=9.95 \mathrm{~Hz}), 4.09(2 \mathrm{H}, \mathrm{q}, J=7.00 \mathrm{~Hz}), 3.37(3 \mathrm{H}, \mathrm{s}), 1.41 \mathrm{ppm}(3 \mathrm{H}, \mathrm{t}$, $J=7.15 \mathrm{~Hz}) ;{ }^{13} \mathrm{C}$ NMR $\left(125 \mathrm{MHz}, \mathrm{CDCl}_{3}\right) 184.64,178.16,163.66$, $156.66,150.94,145.68,133.18,124.65,124.47,119.51,108.83$, 105.65, 75.01, 64.37, 52.88, 14.76; $\operatorname{ESIMS~(m/z,\% ):~} 313.5[\mathrm{M}+\mathrm{H}]^{+}$.

6-Ethoxyprotoflavone 1'-O-ethyl ether (61): Yellow solid; yield: 41.2\%; NP-HPLC purity: $98.12 \%,{ }^{1} \mathrm{H}$ NMR $\left(500 \mathrm{MHz}, \mathrm{CDCl}_{3}\right) \delta=7.49$ $(1 \mathrm{H}, \mathrm{d}, J=2.85 \mathrm{~Hz}), 7.26(1 \mathrm{H}, \mathrm{d}, J=9.25 \mathrm{~Hz}), 7.20(1 \mathrm{H}, \mathrm{dd}, J=$ $9.20 \mathrm{~Hz}, 2.90 \mathrm{~Hz}), 6.80(2 \mathrm{H}, \mathrm{d}, J=10.00 \mathrm{~Hz}), 6.78(1 \mathrm{H}, \mathrm{s}, \mathrm{H}-3), 6.50$ $(2 \mathrm{H}, \mathrm{d}, J=10.00 \mathrm{~Hz}), 4.09(2 \mathrm{H}, \mathrm{q}, J=7.00 \mathrm{~Hz}), 3.57(2 \mathrm{H}, \mathrm{q}, J=$ $6.85 \mathrm{~Hz}), 1.41(3 \mathrm{H}, \mathrm{t}, J=6.85 \mathrm{~Hz}), 1.27 \mathrm{ppm}(3 \mathrm{H}, \mathrm{t}, J=6.95 \mathrm{~Hz})$; ${ }^{13} \mathrm{C}$ NMR $\left(125 \mathrm{MHz}, \mathrm{CDCl}_{3}\right) 184.83,178.54,164.14,156.71,151.02$, $146.25,132.68,124.64,124.53,119.55,108.77,105.59,74.71,64.39$, 61.03, 15.78, 14.75; $\operatorname{ESIMS~(m/z,\% ):~} 327.6[M+\mathrm{H}]^{+}$.

6-Ethoxyprotoflavone 1'-O-propyl ether (62): Yellow solid; yield: 45.8\%; NP-HPLC purity: $99.20 \%,{ }^{1} \mathrm{H}$ NMR $\left(500 \mathrm{MHz} \mathrm{CDCl}_{3}\right) \delta=7.49$ $(1 \mathrm{H}, \mathrm{d}, J=2.85 \mathrm{~Hz}), 7.25(1 \mathrm{H}, \mathrm{d}, J=9.25 \mathrm{~Hz}), 7.20(1 \mathrm{H}, \mathrm{dd}, J=$ $9.20 \mathrm{~Hz}, 2.90 \mathrm{~Hz}), 6.79(2 \mathrm{H}, \mathrm{d}, J=9.80 \mathrm{~Hz}), 6.78(1 \mathrm{H}, \mathrm{s}, \mathrm{H}-3), 6.51$ $(2 \mathrm{H}, \mathrm{d}, J=10.10 \mathrm{~Hz}), 4.09(2 \mathrm{H}, \mathrm{q}, J=7.00 \mathrm{~Hz}), 3.46(2 \mathrm{H}, \mathrm{t}, J=$ $6.45 \mathrm{~Hz}), 1.65(2 \mathrm{H}, \operatorname{sex}, J=7.15 \mathrm{~Hz}), 1.41(3 \mathrm{H}, \mathrm{t}, J=6.90 \mathrm{~Hz})$, $0.96 \mathrm{ppm}(3 \mathrm{H}, \mathrm{t}, J=7.40 \mathrm{~Hz}) ;{ }^{13} \mathrm{C}$ NMR $\left(125 \mathrm{MHz}, \mathrm{CDCl}_{3}\right)$ 184.85, $178.31,163.97,156.65,150.95,146.37,132.68,124.65,124.45$, $119.51,108.84,105.63,74.59,66.92,64.37,23.47,14.77,10.7$; ESIMS $(\mathrm{m} / \mathrm{z}, \%): 341.5[\mathrm{M}+\mathrm{H}]^{+}$.

6-Ethoxyprotoflavone 1'-O-isopropyl ether (63): Yellow solid; yield: $43.1 \%$; NP-HPLC purity: $98.20 \%,{ }^{1} \mathrm{H}$ NMR $\left(500 \mathrm{MHz}, \mathrm{CDCl}_{3}\right)$ $\delta=7.48(1 \mathrm{H}, \mathrm{d}, J=2.75 \mathrm{~Hz}), 7.25(1 \mathrm{H}, \mathrm{d}, J=9.00 \mathrm{~Hz}), 7.20(1 \mathrm{H}, \mathrm{dd}$, $J=9.15 \mathrm{~Hz}, 2.85 \mathrm{~Hz}), 6.81(2 \mathrm{H}, \mathrm{d}, J=10.00 \mathrm{~Hz}), 6.80(1 \mathrm{H}, \mathrm{s}), 6.48$ 
$(2 \mathrm{H}, \mathrm{d}, J=9.95 \mathrm{~Hz}), 4.09(2 \mathrm{H}, \mathrm{q}, J=7.00 \mathrm{~Hz}), 3.84(2 \mathrm{H}$, quin, $J=$ $6.15 \mathrm{~Hz}), 1.42(3 \mathrm{H}, \mathrm{t}, J=7.30 \mathrm{~Hz}), 1.21 \mathrm{ppm}(6 \mathrm{H}, \mathrm{d}, J=6.35 \mathrm{~Hz})$; ${ }^{13} \mathrm{C} \mathrm{NMR}\left(125 \mathrm{MHz}, \mathrm{CDCl}_{3}\right)$ 185.05, 178.35, 164.16, 156.64, 150.93, $146.72,131.97,124.64,124.43,119.51,108.96,105.62,74.91,68.93$, 64.36, 24.82, 14.77; $\operatorname{ESIMS~(m/z):~} 341.5[\mathrm{M}+\mathrm{H}]^{+}$.

6-Ethoxyprotoflavone 1'-O-buthyl ether (64): Light-brown solid; yield: $49.9 \%$; NP-HPLC purity: $98.30 \%,{ }^{1} \mathrm{H}$ NMR $\left(500 \mathrm{MHz}, \mathrm{CDCl}_{3}\right)$ $\delta=7.49(1 \mathrm{H}$, brs $), 7.26(1 \mathrm{H}, \mathrm{d}, J=9.10 \mathrm{~Hz}), 7.22(1 \mathrm{H}, \mathrm{dd}, J=$ $8.55 \mathrm{~Hz}, 2.85 \mathrm{~Hz}), 6.83(1 \mathrm{H}, \mathrm{s}), 6.79(2 \mathrm{H}, \mathrm{d}, J=9.55 \mathrm{~Hz}), 6.51(2 \mathrm{H}, \mathrm{d}$, $J=9.60 \mathrm{~Hz}), 4.09(2 \mathrm{H}, \mathrm{q}, J=6.85 \mathrm{~Hz}), 3.84(2 \mathrm{H}, \mathrm{t}, J=6.05 \mathrm{~Hz}), 1.61$ $(2 \mathrm{H}, \mathrm{q}, J=6.65 \mathrm{~Hz}), 1.45-1.32(2 \mathrm{H}, \mathrm{m}), 1.41(3 \mathrm{H}, \mathrm{t}, J=6.40 \mathrm{~Hz})$, $0.92 \mathrm{ppm}(3 \mathrm{H}, \mathrm{t}, J=7.25 \mathrm{~Hz}) ;{ }^{13} \mathrm{C}$ NMR $\left(125 \mathrm{MHz}, \mathrm{CD}_{3} \mathrm{OD}\right) 186.46$, $166.74,158.31,152.45,148.05,133.64,125.79,125.36,120.82$, 109.11, 106.55, 75.99, 66.07, 65.45, 33.38, 20.46, 15.07, 14.32; ESIMS $(\mathrm{m} / \mathrm{z}): 355.2[\mathrm{M}+\mathrm{H}]^{+}$

6-Ethylprotoflavone (65): Light-brown solid; yield: 41.0\%; RPHPLC purity: $95.0 \%,{ }^{1} \mathrm{H}$ NMR $\left(500 \mathrm{MHz}, \mathrm{CDCl}_{3}\right) \delta=7.92(1 \mathrm{H}, \mathrm{s}), 7.48$ $(1 \mathrm{H}, \mathrm{d}, J=8,55 \mathrm{~Hz}), 7.27(1 \mathrm{H}, \mathrm{d}, J=8,65 \mathrm{~Hz}), 6.95(2 \mathrm{H}, \mathrm{d}, J=$ $9,30 \mathrm{~Hz}), 6.86(1 \mathrm{H}, \mathrm{s}), 6.38(2 \mathrm{H}, \mathrm{d}, J=9,30 \mathrm{~Hz}), 2.72(2 \mathrm{H}, \mathrm{q}, J=$ $7.50 \mathrm{~Hz}), 1.25 \mathrm{ppm}(3 \mathrm{H}, \mathrm{t}, J=7.00 \mathrm{~Hz}) ;{ }^{13} \mathrm{C}$ NMR $\left(125 \mathrm{MHz}, \mathrm{CDCl}_{3}\right)$ $185.33,179.31,166.56,154.82,146.63,142.31,134.77,129.89$, 123.88, 123.37, 118.11, 108.68, 69.94, 28.44, 15.58; ESIMS $(\mathrm{m} / \mathrm{z})$ : $283.3[\mathrm{M}+\mathrm{H}]^{+}$

6-Pentylprotoflavone (66): Light-brown solid; yield: 42.3\%; NPHPLC purity: $96.10 \%,{ }^{1} \mathrm{H} N M R\left(500 \mathrm{MHz}, \mathrm{CDCl}_{3}\right) \delta=7.92(1 \mathrm{H}, \mathrm{s})$, $7.46(1 \mathrm{H}, \mathrm{dd}, J=8.45 \mathrm{~Hz}, 1.25 \mathrm{~Hz}), 7.28(1 \mathrm{H}, \mathrm{d}, J=8.95 \mathrm{~Hz}), 6.90$ $(2 \mathrm{H}, \mathrm{d}, J=9.75 \mathrm{~Hz}), 6.79(1 \mathrm{H}, \mathrm{s}), 6.38(2 \mathrm{H}, \mathrm{d}, J=9.80 \mathrm{~Hz}), 2,66(2 \mathrm{H}$, $\mathrm{t}, J=7.60 \mathrm{~Hz}), 1.61(2 \mathrm{H}$, quin, $J=6.90 \mathrm{~Hz}), 1.38-1.21(4 \mathrm{H}, \mathrm{m})$, $0.85 \mathrm{ppm}(3 \mathrm{H}, \mathrm{t}, J=7.05 \mathrm{~Hz}) ;{ }^{13} \mathrm{C}$ NMR $\left(125.7 \mathrm{MHz}, \mathrm{CDCl}_{3}\right) 176.91$ $162.93,160.90,154.00,139.61,134.37,123.38,118.20,104.68,34.37$, 30.48, 20.73, 21.90, 13.88; ESIMS (m/z): $325.4[\mathrm{M}+\mathrm{H}]^{+}$.

6-Phenylprotoflavone (68): Yellow solid; yield: $38.1 \%$; NP-HPLC purity: $95.19 \%,{ }^{1} \mathrm{H} N M R\left(500 \mathrm{MHz}, \mathrm{CDCl}_{3}\right) \delta=8.37(1 \mathrm{H}, \mathrm{d}, J=$ $1.40 \mathrm{~Hz}), 7.88(1 \mathrm{H}, \mathrm{dd}, J=9.00 \mathrm{~Hz}, 1.85 \mathrm{~Hz}), 7.62(2 \mathrm{H}, \mathrm{d}, J=7.55 \mathrm{~Hz})$, $7.45(2 \mathrm{H}, \mathrm{t}, J=6.75 \mathrm{~Hz}), 7.41(1 \mathrm{H}, \mathrm{d}, J=8.95 \mathrm{~Hz}), 7.37(1 \mathrm{H}, \mathrm{t}, J=$ $7.65 \mathrm{~Hz}), 6.91(2 \mathrm{H}, \mathrm{d}, J=10.10 \mathrm{~Hz}), 6.82(1 \mathrm{H}, \mathrm{s}), 6.57 \mathrm{ppm}(2 \mathrm{H}, \mathrm{d}$, $J=9.90 \mathrm{~Hz}) ;{ }^{13} \mathrm{C}$ NMR $\left(50 \mathrm{MHz}, \mathrm{CDCl}_{3}\right) \delta=145.07,131.64,128.27$, $127.94,127.42,126.95,126.10,122.51,122.51,117.47,108.50$, 65.77 ppm; HRESIMS $\mathrm{C}_{21} \mathrm{H}_{15} \mathrm{O}_{4}$, calcd: 331.0970 , found: 331.0973 .

6-Phenylprotoflavone 1'-O-methyl ether (69): Yellow solid; yield: $50.2 \%$; NP-HPLC purity: $97.74 \%,{ }^{1} \mathrm{H}$ NMR $\left(500 \mathrm{MHz}, \mathrm{CDCl}_{3}\right) \delta=8.37$ $(1 \mathrm{H}, \mathrm{d}, J=1.50 \mathrm{~Hz}), 7.88(1 \mathrm{H}, \mathrm{dd}, J=8.35 \mathrm{~Hz}, 1.50 \mathrm{~Hz}), 7.66(2 \mathrm{H}, \mathrm{d}$, $J=7.85 \mathrm{~Hz}), 7.45(2 \mathrm{H}, \mathrm{t}, J=7,10 \mathrm{~Hz}), 7.41(1 \mathrm{H}, \mathrm{d}, J=8.85 \mathrm{~Hz}), 7.37$ $(1 \mathrm{H}, \mathrm{t}, J=7.00 \mathrm{~Hz}), 6.82(2 \mathrm{H}, \mathrm{d}, J=9.95 \mathrm{~Hz}), 6.77(1 \mathrm{H}, \mathrm{s}), 6.57 \mathrm{ppm}$ $(2 \mathrm{H}, \mathrm{d}, J=9.90 \mathrm{~Hz}), 3.41(3 \mathrm{H}, \mathrm{s}) ;{ }^{13} \mathrm{C}$ NMR $\left(50 \mathrm{MHz}, \mathrm{CDCl}_{3}\right) \delta=$ $144.39,132.14,131.81,128.26,127.94,127.42,127.26,126.92$, $126.10,122.51,121.72,117.47,108.49,108.11,51.71,28.63$ ppm; HRESIMS $\mathrm{C}_{22} \mathrm{H}_{17} \mathrm{O}_{4}$, calcd: 345.1127 , found: 345.1125 .

6-Phenylprotoflavone 1 '-O-ethyl ether (70): Yellow solid; yield: $48.2 \%$; NP-HPLC purity: $98.10 \%$, $1 \mathrm{H} \mathrm{NMR}\left(500 \mathrm{MHz}, \mathrm{CDCl}_{3}\right) \delta=8.37$ $(1 \mathrm{H}, \mathrm{d}, J=1.50 \mathrm{~Hz}), 7.87(1 \mathrm{H}, \mathrm{d}, J=8.60 \mathrm{~Hz}), 7.62(2 \mathrm{H}, \mathrm{d}, J=$ $7.50 \mathrm{~Hz}), 7.44(2 \mathrm{H}, \mathrm{t}, J=7.20 \mathrm{~Hz}), 7.40(1 \mathrm{H}, \mathrm{d}, J=8.85 \mathrm{~Hz}), 7.36(1 \mathrm{H}$, brt $J=6.75 \mathrm{~Hz}), 6.84(1 \mathrm{H}, \mathrm{s}), 6.83(2 \mathrm{H}, \mathrm{d}, J=10.10 \mathrm{~Hz}), 6.53(2 \mathrm{H}, \mathrm{d}$, $J=9.80 \mathrm{~Hz}), 3.58(2 \mathrm{H}, \mathrm{q}, J=7.00 \mathrm{~Hz}), 1.28 \mathrm{ppm}(3 \mathrm{H}, \mathrm{t}, J=6.80 \mathrm{~Hz})$; ${ }^{13} \mathrm{C} \mathrm{NMR}\left(50 \mathrm{MHz}, \mathrm{CDCl}_{3}\right) \delta=144.84,132.63,131.78,128.29,127.96$, $127.42,127.24,126.91,126.10,122.51,121.70,117.49,108.52$, $108.15, \quad 59.85,28.63,14.60$ ppm; HRESIMS $\mathrm{C}_{23} \mathrm{H}_{19} \mathrm{O}_{4}$, calcd: 359.1283 , found: 359.1280 .

6-Phenylprotoflavone 1'-O-propyl ether (71): Light-brown solid; yield: 46.4\%; NP-HPLC purity: $99.39 \%$, ${ }^{1} \mathrm{H}$ NMR $\left(500 \mathrm{MHz} \mathrm{CDCl}_{3}\right)$ $\delta=8.37(1 \mathrm{H}, \mathrm{d}, J=1.40 \mathrm{~Hz}), 7.88(1 \mathrm{H}, \mathrm{dd}, J=8.85 \mathrm{~Hz}, 1.60 \mathrm{~Hz}), 7.62$ $(2 \mathrm{H}, \mathrm{d}, J=7.50 \mathrm{~Hz}), 7.44(2 \mathrm{H}, \mathrm{brt}, J=6.45 \mathrm{~Hz}), 7.40(1 \mathrm{H}, \mathrm{d}, J=$ $8.70 \mathrm{~Hz}), 7.36(1 \mathrm{H}, \mathrm{t}, J=7.20 \mathrm{~Hz}), 6.84(1 \mathrm{H}, \mathrm{s}), 6.83(2 \mathrm{H}, \mathrm{d}, J=$ $9.95 \mathrm{~Hz}), 6.54(2 \mathrm{H}, \mathrm{d}, J=9.90 \mathrm{~Hz}), 3.48(2 \mathrm{H}, \mathrm{t}, J=6.25 \mathrm{~Hz}), 1.67(2 \mathrm{H}$, sex, $J=6.95 \mathrm{~Hz}), 0.98 \mathrm{ppm}(3 \mathrm{H}, \mathrm{t}, J=7.35 \mathrm{~Hz}) ;{ }^{13} \mathrm{C} \mathrm{NMR}(50 \mathrm{MHz}$, $\left.\mathrm{CDCl}_{3}\right) \delta=145.07,131.64,128.27,127.94,127.42,126.95,126.10$ $122.51,117.47,108.50,65.77,30.89,28.63,22.27,9.51$ ppm; HRESIMS $\mathrm{C}_{24} \mathrm{H}_{21} \mathrm{O}_{4}$, calcd: 373.1440 , found: 373.1438 .

6-Phenylprotoflavone 1'-O-isopropyl ether (72): Light-brown solid; yield: $42.2 \%$; NP-HPLC purity: $99.30 \%,{ }^{1} \mathrm{H} \mathrm{NMR}(500 \mathrm{MHz}$, $\left.\mathrm{CDCl}_{3}\right) \delta=8.37(1 \mathrm{H}, \mathrm{brs}), 7.88(1 \mathrm{H}, \mathrm{dd}, J=8.10 \mathrm{~Hz}, 1.25 \mathrm{~Hz}), 7.62$ $(2 \mathrm{H}, \mathrm{d}, J=7.85 \mathrm{~Hz}), 7.45(2 \mathrm{H}, \mathrm{brt}, J=6.65 \mathrm{~Hz}), 7.41(1 \mathrm{H}, \mathrm{d}, J=$ $8.85 \mathrm{~Hz}), 7.37(1 \mathrm{H}, \mathrm{t}, J=7.00 \mathrm{~Hz}), 6.82(2 \mathrm{H}, \mathrm{d}, J=9.95 \mathrm{~Hz}), 6.77(1 \mathrm{H}$, s), $6.57(2 \mathrm{H}, \mathrm{d}, J=9.90 \mathrm{~Hz}), 3,89 \mathrm{ppm}(1 \mathrm{H}$, sext, $J=6.25 \mathrm{~Hz})$; ${ }^{13} \mathrm{C} \mathrm{NMR}\left(50 \mathrm{MHz}, \mathrm{CDCl}_{3}\right) \delta=145.44,138.05,132.61,128.30,127.96$, $127.44,127.24,126.91,126.10,122.51,121.70,117.47,108.62$, 108.26, 97.24, 67.81, 28.63, 23.62 ppm; HRESIMS $\mathrm{C}_{24} \mathrm{H}_{21} \mathrm{O}_{4}$, calcd: 373.1440, found: 373.1439 .

6-Phenylprotoflavone 1'-O-butyl ether (73): Light-brown solid; yield: $50.1 \%$; NP-HPLC purity: $95.4 \%$; ${ }^{1} \mathrm{H}$ NMR $\left(200 \mathrm{MHz}, \mathrm{CDCl}_{3}\right) \delta=$ $8.40(1 \mathrm{H}, \mathrm{d}, J=1.50 \mathrm{~Hz}), 7.89(1 \mathrm{H}, \mathrm{dd}, J=9.02 \mathrm{~Hz}, 2.00 \mathrm{~Hz}), 7.64(2 \mathrm{H}$, $\mathrm{d}, J=8.00 \mathrm{~Hz}), 7.47(2 \mathrm{H}, \mathrm{brt}, J=6.60 \mathrm{~Hz}), 7.41(1 \mathrm{H}, \mathrm{d}, J=8.20 \mathrm{~Hz})$, $7.39(1 \mathrm{H}, \mathrm{t}, J=7.00 \mathrm{~Hz}), 6.81(1 \mathrm{H}, \mathrm{s}), 6.56(2 \mathrm{H}, \mathrm{d}, J=10.00 \mathrm{~Hz}), 6.39$ $(2 \mathrm{H}, \mathrm{d}, J=9.80 \mathrm{~Hz}), 3.54(2 \mathrm{H}, \mathrm{t}, J=5.80 \mathrm{~Hz}), 1.68-1.36(4 \mathrm{H}, \mathrm{m})$, $0.95 \mathrm{ppm}(3 \mathrm{H}, \mathrm{t}, J=7.20 \mathrm{~Hz}) ;{ }^{13} \mathrm{C}$ NMR $\left(50 \mathrm{MHz}, \mathrm{CDCl}_{3}\right) \delta=145.01$, $138.03,132.63,128.27,127.96,127.42,127.24,126.91,126.10,122.52$, $121.72,117.47,108.52,108.15,63.89,31.01,28.63,18.20$ ppm; HRESIMS $\mathrm{C}_{25} \mathrm{H}_{23} \mathrm{O}_{4}$, calcd: 387.1596 , found: 387.1593 .

\section{Cell lines}

L5178 mouse T-cell lymphoma cell line (ECACC cat. no. 87111908, US FDA, Silver Spring, MD, USA), and its sub-cell line $L 5178_{B 1}$, derived from $\mathrm{L} 5178$ by transfection with $\mathrm{pHa}$ MDR1/A retrovirus, ${ }^{[26]}$ were cultured in McCoy's 5A media supplemented with inactivated horse serum. $L 5178_{B 1}$ cell line was selected by culturing the infected cells with colchicine $\left(60 \mu \mathrm{g} \mathrm{L}^{-1}\right.$, Sigma). Breast cancer cell lines MCF7 and its sub-cell line obtained by adaptation to doxorubicin, MCF7 $_{\text {Dox }}{ }^{[27]}$ were cultured in EMEM media supplemented with nonessential amino acids, Na-pyruvate $(1 \mathrm{~mm})$ and inactivated fetal bovine serum (FBS, $10 \%$; MCF7 Dox was cultured in presence of doxorubicin $(1 \mu \mathrm{M})$ each third passage). All above cell lines were cultured at $37^{\circ} \mathrm{C}$ and $5 \% \mathrm{CO}_{2}$; all media contained Nystatin, L-glutamine $(2 \mathrm{~mm})$, penicillin $(100 \mathrm{U})$ and streptomycin $(0.1 \mathrm{mg})$, purchased from Sigma. MES-SA human uterine sarcoma cell line and the doxorubicin selected MES-SA/Dx5 were obtained from ATCC. The human cervix carcinoma cell line KB-3-1 and its vinblastine selected derivative KB-V1 were a kind gift from Dr. Michael M. Gottesman (US National Institutes of Health). A431 and the retrovirally transduced $A 431_{B 1}$ and $A 431_{G 2}$ are human skin-derived, epidermoid carcinoma cells were kind gifts from Dr. K. Német. MES-SA, KB-3-1, A431 and their derivative cell lines were maintained in DMEM completed with FBS (10\%), glutamine $(5 \mathrm{~mm})$ and penicillin and streptomycin $50 \mathrm{U} \mathrm{mL}^{-1}$, (Life Technologies).

\section{Cytotoxicity assays}

In the case of the mouse lymphoma cell lines $L 5178$ and $L 5178_{B 1}$, $2 \times 10^{4}$ cells per well were cultured in 96-wells microplates with different concentrations of the tested compound, in McCoy's $5 \mathrm{~A}$ media, at $37^{\circ} \mathrm{C}$ and $5 \% \mathrm{CO}_{2}$, for $24 \mathrm{~h}$. With respect to MCF7 and MCF $_{\text {Dox, }} 1 \times 10^{4}$ cells per well were seeded overnight $(\mathrm{o} / \mathrm{n})$ and 
serial dilutions of the compounds were added the following day and incubated for $48 \mathrm{~h}$. In all cases, after the incubation time, MT $(10 \%)$ was added to each well and incubated for $4 \mathrm{~h}$, when sodium dodecyl-sulfate (SDS, $100 \mu \mathrm{M}$ of $10 \%$ ) dissolved in $\mathrm{HCl}$ $(0.01 \mathrm{M})$ was added to each well. Results were read after o/n incubation. Fifty per cent inhibitory concentrations $\left(\mathrm{IC}_{50}\right)$ were calculated using nonlinear regression curve fitting of log (inhibitor) versus normalized response with a variable slope and least squares (ordinary) fit of GraphPad Prism 5 software, for three independent samples. In the case of A431 cell lines, MES-SA cell lines, KB-3-1 and $\mathrm{KB}-\mathrm{V} 1$ cell lines, $5 \times 10^{3}$ cells per well were cultured and incubated $\mathrm{o} / \mathrm{n}$ in $96-$ well microplates. Serially diluted drugs were then added, and plates were incubated for additional $72 \mathrm{~h}$. Cytotoxicity was measured by Presto Blue cell viability reagent (Invitrogen) in a final dilution of $5 \%$.

\section{FACS measurements}

Calcein accumulation assay was performed as described earlier. ${ }^{[21]}$ Briefly, 250000 cells per tube were pre-incubated for 5 minutes in the presence of verapamil $(20 \mu \mathrm{M})$ or the test protoflavone compounds. Calcein AM was added at a final concentration of $250 \mathrm{~nm}$ and incubated for an additional 10 minutes. Samples were then washed with ice-cold PBS and were kept on ice until measured by an Attune Acoustic Focusing Cytometer.

\section{Supporting information available}

Cytotoxicity data on the studied cancer cell line pairs and corresponding selectivity ratios, as well as data of the calcein accumulation assay are presented as Tables S1-S4, and correlation of $\mathrm{IC}_{50}$ values on the $\left[5178_{B 1}\right.$, MCF-7 and MCF- $7_{\text {Dox }}$ cell lines with those on the $\mathrm{L} 5178$ cells as Figure S1.

\section{Acknowledgements}

This work was supported by the National Research, Development and Innovation Office, Hungary (NKFIH; K119770). A.H. acknowledges the János Bolyai fellowship of the Hungarian Academy of Sciences, the Kálmán Szász Prize and Networking contribution from COST Actions CM1106 (Chemical Approaches to Targeting Drug Resistance in Cancer Stem Cells) and CM1407 (Challenging organic syntheses inspired by nature-from natural products chemistry to drug discovery). G.S. was supported by ERC (StG260572), TÉT_13_DST-1-2013-0012 and a Momentum Grant of the Hungarian Academy of Sciences. Support from the National Science Council (NSC), Taiwan and the Szeged Foundation for Cancer Research, as well as a bilateral mobility grant from the Hungarian Academy of Sciences and the NSC Taiwan is acknowledged. B.D. acknowledges Zoltán Ujlaki for his efforts in synthesizing compounds 59-64 and Dr. Da-Wei Chuang (Graduate Institute of Natural Products, KMU, Kaohsiung, Taiwan) for scientific discussion.

\section{Conflict of interest}

The authors declare no conflict of interest.
Keywords: collateral sensitivity • cross-resistance - MDR cancer $\cdot$ P-glycoprotein $\cdot$ protoflavones

[1] WHO Factsheet No. 297, February 2017, World Health Organization, available at http://www.who.int/mediacentre/factsheets/fs297/en/ (accessed February 1, 2017).

[2] C. Holohan, S. Van Schaeybroeck, D. B. Longley, P. G. Johnston, Nat. Rev. Cancer 2013, 13, 714-726.

[3] P. Borst, Open Biol. 2012, 2, 120066.

[4] M. M. Gottesman, T. Fojo, S. E. Bates, Nat. Rev. Cancer 2002, 2, 48-58.

[5] G. Szakács, J. K. Paterson, J. A. Ludwig, C. Booth-Genthe, M. M. Gottesman, Nat. Rev. Drug Discovery 2006, 5, 219-234.

[6] G. Szakács, M. D. Hall, M. M. Gottesman, A. Boumendjel, R. Kachadourian, B. J. Day, H. Baubichon-Cortay, A. Di Pietro, Chem. Rev. 2014, 114, $5753-5774$.

[7] M. Baym, L. K. Stone, R. Kishony, Science 2016, 351, aad3292.

[8] J. A. Ludwig, G. Szakács, S. E. Martin, B. F. Chu, C. Cardarelli, Z. E. Sauna, N. J. Caplen, H. M. Fales, S. V. Ambudkar, J. N. Weinstein, M. M. Gottesman, Cancer Res. 2006, 66, 4808-4815.

[9] G. Szakács, J. P. Annereau, S. Lababidi, U. Shankavaram, A. Arciello, K. J. Bussey, W. Reinhold, Y. Guo, G. D. Kruh, M. Reimers, J. N. Weinstein, M. M. Gottesman, Cancer Cell 2004, 6, 129-137.

[10] A. Füredi, S. Tóth, K. Szebényi, V. F. S. Pape, D. Türk, N. Kucsma, L. Cervenák, J. Tóvári, G. Szakács, Mol. Cancer Ther. 2017, 16, 35-44.

[11] R. M. Laberge, J. Karwatsky, M. C. Lincoln, M. L. Leimanis, E. Georges, Biochem. Pharmacol. 2007, 73, 1727-1737.

[12] A. Hunyadi, A. Martins, B. Danko, F. R. Chang, Y. C. Wu, Phytochem. Rev. 2014, 13, 69-77.

[13] W. Y. Chen, Y. A. Hsieh, C. I. Tsai, Y. F. Kang, F. R. Chang, Y. C. Wu, C. C. Wu, Invest. New Drugs 2011, 29, 1347-1359.

[14] H. C. Wang, A. Y. Lee, W. C. Chou, C. C. Wu, C. N. Tseng, K. Y. Liu, W. L. Lin, F. R. Chang, D. W. Chuang, A. Hunyadi, Y. C. Wu, Mol. Cancer Ther. 2012, 11, 1443-1453.

[15] B. Danko, A. Martins, D. W. Chuang, H. C. Wang, L. Amaral, J. Molnar, F. R. Chang, Y. C. Wu, A. Hunyadi, Anticancer Res. 2012, 32, 2863-2870.

[16] T. Stanković, B. Dankó, A. Martins, M. Dragoj, S. Stojković, A. Isaković, H. C. Wang, Y. C. Wu, A. Hunyadi, M. Pešić, Cancer Chemother. Pharmacol. 2015, 76, 555-565.

[17] A. Hunyadi, D. W. Chuang, B. Danko, M. Y. Chiang, C. L. Lee, H. C. Wang, C. C. Wu, F. R. Chang, Y. C. Wu, PLoS One 2011, 6, e23922.

[18] Q. D. Tu, D. Li, Y. Sun, X. Y. Han, F. Yi, Y. Sha, Y. L. Ren, M. W. Ding, L. L. Feng, J. Wan, Bioorg. Med. Chem. 2013, 21, 2826-2831.

[19] W. G. Harker, B. I. Sikic, Cancer Res. 1985, 45, 4091-4096.

[20] D. W. Shen, C. Cardarelli, J. Hwang, M. Cornwell, N. Richert, S. Ishii, I. Pastan, M. M. Gottesman, J. Biol. Chem. 1986, 261, 7762-7770.

[21] L. Homolya, M. Hollo, M. Muller, E. B. Mechetner, B. Sarkadi, Br. J. Cancer 1996, 73, 849-855.

[22] A. S. Lin, K. Nakagawa-Goto, F. R. Chang, D. Yu, S. L. Morris-Natschke, C. C. Wu, S. L. Chen, Y. C. Wu, K. H. Lee, J. Med. Chem. 2007, 50, 3921 3927.

[23] L. Rickardson, M. Fryknäs, C. Haglund, H. Lövborg, P. Nygren, M. G. Gustafsson, A. Isaksson, R. Larsson, Cancer Chemother. Pharmacol. 2006, 58, $749-758$.

[24] A. S. Goldsborough, M. D. Handley, A. E. Dulcey, K. M. Pluchino, P. Kannan, K. R. Brimacombe, M. D. Hall, G. Griffiths, M. M. Gottesman, J. Med. Chem. 2011, 54, 4987-4997.

[25] D. Türk, M. D. Hall, B. F. Chu, J. A. Ludwig, H. M. Fales, M. M. Gottesman, G. Szakács, Cancer Res. 2009, 69, 8293-8301.

[26] I. Pastan, M. M. Gottesman, K. Ueda, E. Lovelace, A. V. Rutherford, M. C. Willingham, Proc. Natl. Acad. Sci. USA 1988, 85, 4486-4490.

[27] M. D. Kars, O. D. Iseri, U. Gündüz, A. U. Ural, F. Arpaci, J. Molnár, Anticancer Res. 2006, 26, 4559-4568.

Manuscript received: April 8, 2017

Accepted manuscript online: April 23, 2017

Version of record online: May 23, 2017 